University of Tennessee Health Science Center UTHSC Digital Commons

\title{
Investigation of Narrow Spectrum Targets in Antibacterial Drug Discovery
}

Jesse Jones

University of Tennessee Health Science Center

Follow this and additional works at: https://dc.uthsc.edu/dissertations

Part of the Pharmacy and Pharmaceutical Sciences Commons

\section{Recommended Citation}

Jones, Jesse (0000-0001-8006-5661), "Investigation of Narrow Spectrum Targets in Antibacterial Drug Discovery" (2019). Theses and Dissertations (ETD). Paper 498. http://dx.doi.org/10.21007/ etd.cghs.2019.0491.

This Dissertation is brought to you for free and open access by the College of Graduate Health Sciences at UTHSC Digital Commons. It has been accepted for inclusion in Theses and Dissertations (ETD) by an authorized administrator of UTHSC Digital Commons. For more information, please contact jwelch30@uthsc.edu. 


\title{
Investigation of Narrow Spectrum Targets in Antibacterial Drug Discovery
}

\begin{abstract}
Background: Significant concerns are associated with the use of broad-spectrum antibacterial agents, including collateral eradication of beneficial bacteria from the human microbiome, the onset of antibacterial-associated infections, and continued emergence of antibacterial drug resistance. As such, a critical need for novel and selective antibacterial targets exists. The investigation of two such targets, each pertaining to the highly concerning infections caused by streptococcal species and Clostridioides difficile, are presented herein. Bacterial topoisomerase I represents a potentially promising narrowspectrum target as studies have arisen demonstrating its essentiality in bacterial species lacking the only other type IA topoisomerase (topoisomerase III). Additionally, recent studies demonstrating the essentiality of the fabK gene expressing enoyl-ACP reductase II (FabK) in C. difficile indicate its significant potential as a narrow-spectrum target. Presented here are data characterizing and validating both the Topol and FabK enzymes as novel antibacterial targets via the implementation of an array of drug discovery techniques, including structural studies, biochemical assay development and application, and inhibitor screening and testing. Methods: An assortment of drug discovery techniques were employed for the targeting of SmTopol and CdFabK, including different protein expression and purification techniques; X-ray crystallography; various biophysical and biochemical techniques for target characterization, validation, and drug screening; and different lead development and optimization studies. Results: The respective genes for SmTopol and CdFabK have been cloned, and the expression and purification of various constructs of each target have been carried out and optimized for further analysis. The crystal structure of SmTopol_N65 has been determined to $2.06 \AA$ and diffracting CdFabK crystals $(3.5 \AA)$ have been attained. A high-throughput plate-based biochemical fluorescence kinetic assay has been optimized for screening against the CdFabK enzyme. Furthermore, activity and modality of inhibition assessment of small-molecule inhibitors of the CdFabK enzyme have been conducted, including phenylimidazole and benzothiazole compounds. Phenylimidazole analogues have been found to display micromolar inhibitory activity against CdFabK, and a benzothiazole analogue has been found to display nanomolar inhibitory activity against the target. Conclusions: The SmTopol and CdFabK enzymes present potentially novel, narrow- spectrum antibacterial drug targets, and substantial progress has been made toward the rational targeting of these two enzymes. Of particular note, the first structure of a Topo I fragment from a grampositive organism, S. mutans, has been determined. Enzymology and inhibitor studies have been conducted supporting the druggability of CdFabK and indicating the potential for selective inhibition of CdFabK.
\end{abstract}

Document Type

Dissertation

Degree Name

Doctor of Philosophy (PhD)

Program

Pharmaceutical Sciences

Research Advisor

Hevener, Kirk

Keywords

Antibacterial, Clostridioides difficile, Enoyl-ACP reductase, FabK, Narrow-spectrum, Topoisomerase I 


\section{Subject Categories}

Medicine and Health Sciences | Pharmacy and Pharmaceutical Sciences 


\section{Investigation of Narrow Spectrum Targets in Antibacterial Drug Discovery}

Author:

Jesse Jones
Advisor:

Kirk E. Hevener, Ph.D.

A Dissertation Presented for The Graduate Studies Council of

The University of Tennessee Health Science Center

in Partial Fulfillment of the Requirements for the

Doctor of Philosophy degree from

The University of Tennessee

in

Pharmaceutical Sciences: Bioanalytical Chemistry

College of Graduate Health Sciences 
Portions of Chapter 1 (C) 2016 by The Royal Society of Chemistry.

Portions of Chapter 2 (C) 2016 by Elsevier Inc.

Portions of Chapters 3 and 4 (C) 2019 by American Chemical Society.

All other material (C) 2019 by Jesse Jones.

All rights reserved. 


\section{DEDICATION}

This work is dedicated with love to the amazing women in my life: first of all, my mom, Rose, who taught me the importance of integrity and the unmistakable difference between right and wrong; my step-mom, Debbie, who demonstrated the beauty and grace involved in caring for others, especially when you're not necessarily obligated to; my mother-in-law, Marion, who exemplified the remarkable strength involved in sacrifice; and my wife, Rebecca, who showed me what a person who embodies all of these values simultaneously is really capable of accomplishing - and while doing so, both allowed and inspired me to get this far. 


\section{ACKNOWLEDGEMENTS}

It is difficult for me to fully express the enormous amount of gratitude I feel toward my mentor, Dr. Kirk E. Hevener. He has taught me so much inside the classroom and lab that I will be forever grateful for the knowledge he has shared with me. He reignited my passion for learning when, deep down, I worried it was beginning to sputter out. In doing so, he also taught me to strive to be "academically fearless," and instilled in me the tenacious belief that no target is undruggable. Moreover, he has shown me the importance of sacrificing a little bit of ourselves so that the next generation of healthcare professionals and researchers is able to pick up the torch of knowledge where we one day leave it, carry it a while themselves, and hopefully pass it on again, both burning a little brighter and a little farther down the road. I also firmly believe that his commitment to ethics and knowledge knows no equal—qualities I am sure have made me a better version of myself inside and outside of the lab. Thank you.

I would also like to take the time to thank all of the other members of my committee: Dr. Sarka Beranova-Giorgianni, Dr. Isaac O. Donkor, Dr Eric J. Enemark, and Dr. C. Ryan Yates. All of these professors sacrificed substantial amounts of their time and energy - both of which I am certain are at a premium - and helped guide and motivate me along the way. Without their expertise, examples, general coaching, and numerous solicited recommendations, I would not have been half as successful in graduate school as I have thus far been.

Lastly, I would like to express my appreciation to those funding bodies who helped support my graduate education and research. First, I am grateful to the American Foundation for Pharmaceutical Education for offering me the AFPE Pre-doctoral Fellowship to support my research and allow me to present it to my peers. Their commitment to the advancement and dispersal of knowledge and education stands as a bright reminder of the importance of sharing our knowledge and research successes with others. And finally, I am grateful to the Center for Pediatric Experimental Therapeutics (CPET) at The University of Tennessee Health Science Center (UTHSC) for offering me the CPET scholarship, thereby supporting and encouraging my research and overall education. 


\begin{abstract}
Background: Significant concerns are associated with the use of broad-spectrum antibacterial agents, including collateral eradication of beneficial bacteria from the human microbiome, the onset of antibacterial-associated infections, and continued emergence of antibacterial drug resistance. As such, a critical need for novel and selective antibacterial targets exists. The investigation of two such targets, each pertaining to the highly concerning infections caused by streptococcal species and Clostridioides difficile, are presented herein. Bacterial topoisomerase I represents a potentially promising narrow-spectrum target as studies have arisen demonstrating its essentiality in bacterial species lacking the only other type IA topoisomerase (topoisomerase III). Additionally, recent studies demonstrating the essentiality of the fabK gene expressing enoyl-ACP reductase II (FabK) in $C$. difficile indicate its significant potential as a narrow-spectrum target. Presented here are data characterizing and validating both the TopoI and FabK enzymes as novel antibacterial targets via the implementation of an array of drug discovery techniques, including structural studies, biochemical assay development and application, and inhibitor screening and testing.
\end{abstract}

Methods: An assortment of drug discovery techniques were employed for the targeting of SmTopoI and CdFabK, including different protein expression and purification techniques; X-ray crystallography; various biophysical and biochemical techniques for target characterization, validation, and drug screening; and different lead development and optimization studies.

Results: The respective genes for SmTopoI and CdFabK have been cloned, and the expression and purification of various constructs of each target have been carried out and optimized for further analysis. The crystal structure of SmTopoI_N65 has been determined to $2.06 \AA$ and diffracting CdFabK crystals $(3.5 \AA)$ have been attained. A high-throughput plate-based biochemical fluorescence kinetic assay has been optimized for screening against the CdFabK enzyme. Furthermore, activity and modality of inhibition assessment of small-molecule inhibitors of the CdFabK enzyme have been conducted, including phenylimidazole and benzothiazole compounds. Phenylimidazole analogues have been found to display micromolar inhibitory activity against $\mathrm{CdFabK}$, and a benzothiazole analogue has been found to display nanomolar inhibitory activity against the target.

Conclusions: The SmTopoI and CdFabK enzymes present potentially novel, narrowspectrum antibacterial drug targets, and substantial progress has been made toward the rational targeting of these two enzymes. Of particular note, the first structure of a Topo I fragment from a gram-positive organism, $S$. mutans, has been determined. Enzymology and inhibitor studies have been conducted supporting the druggability of CdFabK and indicating the potential for selective inhibition of $\mathrm{CdFabK}$. 


\section{TABLE OF CONTENTS}

CHAPTER 1. INTRODUCTION ......................................................................................

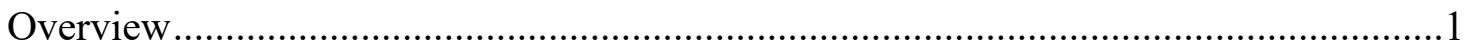

Antibacterial Drug Discovery Eras: Past, Present, and Future .................................. 1

The Golden Era ..................................................................................................... 1

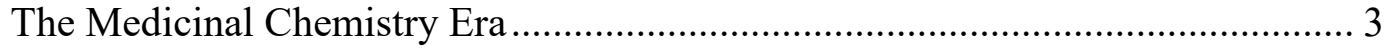

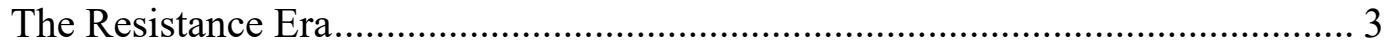

The Post-Antibiotic Era ............................................................................ 3

Public and Private Responses to the Threat of Antimicrobial Resistance ...................4

Recent Advances in Antibacterial Drug Discovery, the Modern Era .........................5

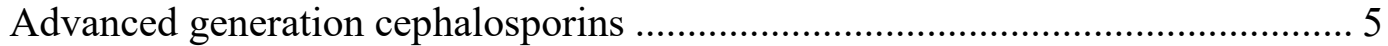

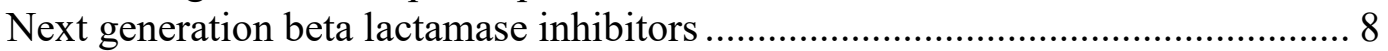

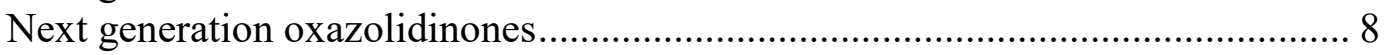

Next generation bacterial topoisomerase inhibitors....................................... 8

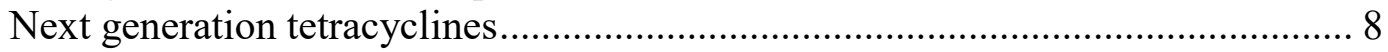

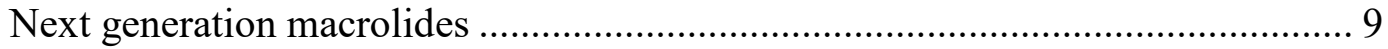

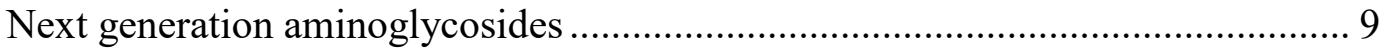

Novel bacterial folate synthesis inhibitor ..................................................... 9

Novel inhibitors of the bacterial FAS-II pathway............................................ 9

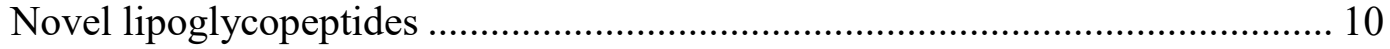

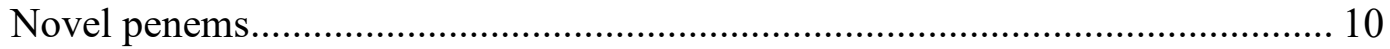

Novel monobactam ............................................................................... 10

Other novel agents ............................................................................... 10

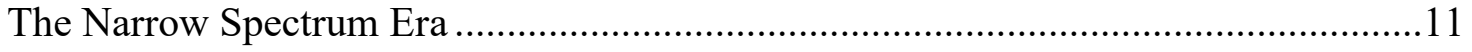

Requisite Antibacterial Drug Discovery Skills and Techniques.............................12

Target identification and production techniques............................................... 12

Preliminary target characterization ............................................................. 14

Compound screening and hit selection techniques ...................................... 15

Target and hit validation and characterization techniques................................. 17

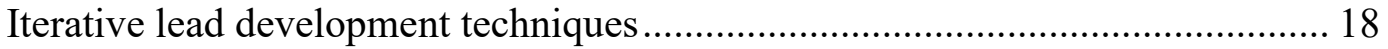

Novel Narrow Spectrum Antibacterial Targets ....................................................19

Bacterial topoisomerase I.................................................................... 20

Bacterial enoyl-acyl carrier protein (ACP) reductase II, FabK ........................ 21

CHAPTER 2. TARGETING BACTERIAL TOPOISOMERASE I...........................22

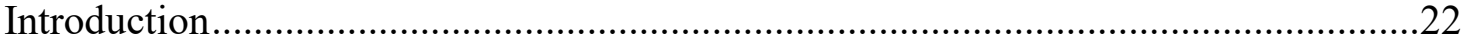

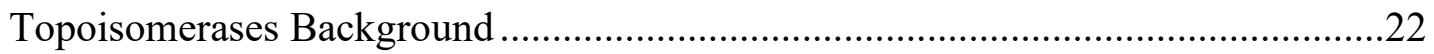

Biological role of topoisomerases........................................................... 22

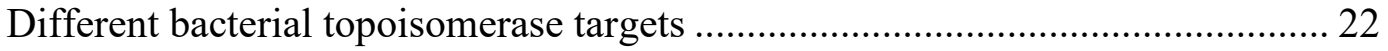

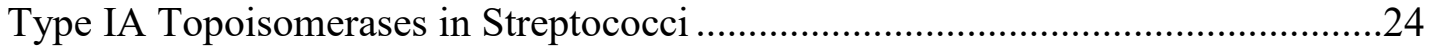

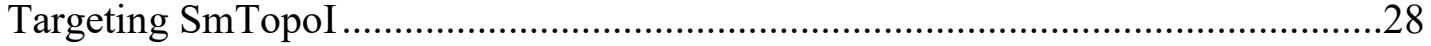

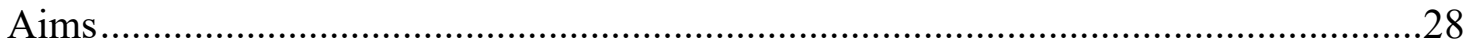

Protein Expression and Purification of SmTopoI ................................................28

Structural Determination of SmTopoI_N65 .......................................................29 
Materials and Methods..............................................................................................

Reagents, Chemicals, Biologicals, and Equipment....................................................30

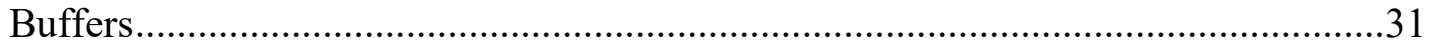

SmTopoI Cloning and Plasmid Construction ............................................................

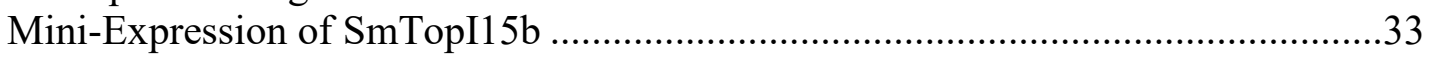

Expression of SmTopoI constructs and cell lysis ......................................................33

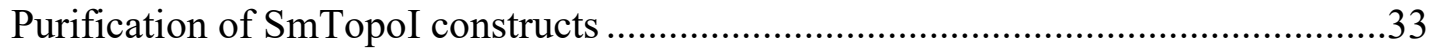

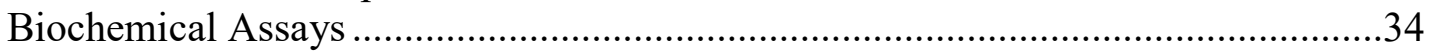

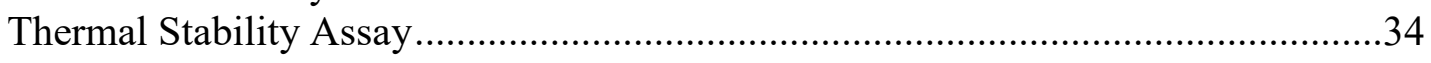

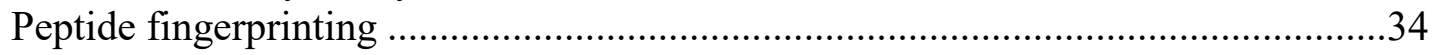

SmTopoI Protein Homology Modeling ....................................................................

SmTopoI_N65 Cloning, Expression, Purification and Crystallization.........................35

SmTopoI_N65 Structure Determination and Refinement ..........................................36

TopoI Sequence and Structure Alignments .............................................................36

Generation of SmTopoI_N65 Topology Model.........................................................36

SmTopoI_N65 Multiple Sequence Alignment with Select Topo I Homologs of

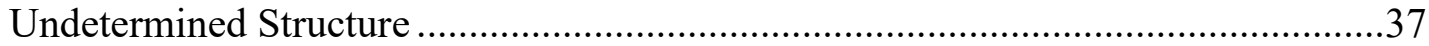

Structural Alignment of SmTopoI_N65 and TmTopoI (PDB 2GUI) ..........................37

SmTopoI_N65 Sequence Alignment with EcTopoIII (PDB 1D6M)...........................37

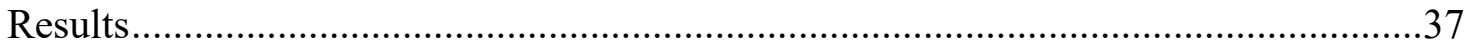

Cloning and Expression of Recombinant SmTopoI ……….....................................37

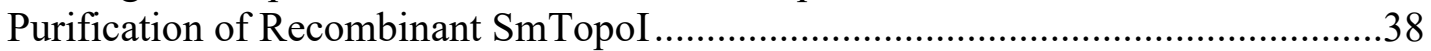

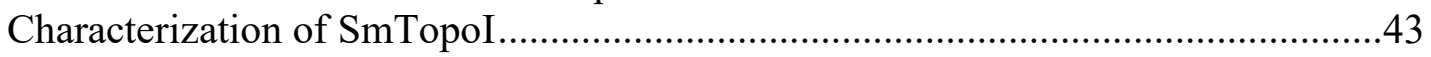

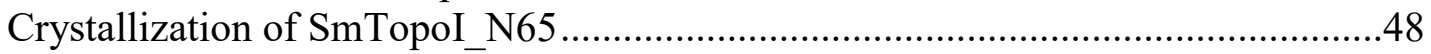

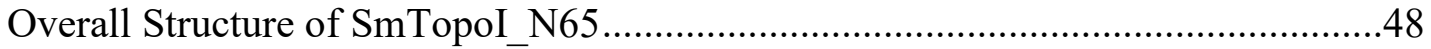

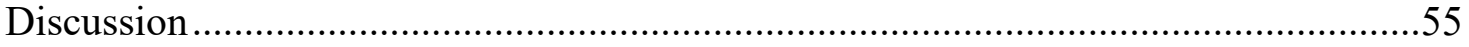

Expression and Purification of SmTopoI ...............................................................55

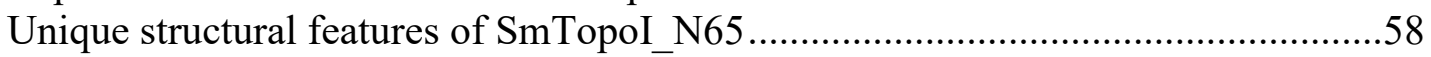

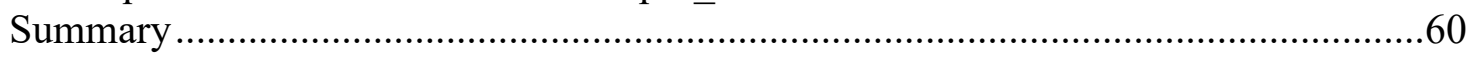

\section{CHAPTER 3. TARGETING ACYL CARRIER PROTEIN (ACP)-ENOYL REDUCTASE II (FABK): PRODUCTION, STABILIZATION, AND

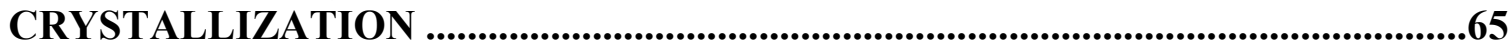

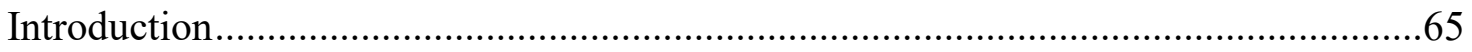

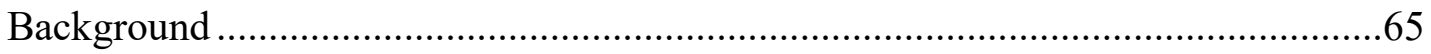

Bacterial Type II Fatty Acid Biosynthesis (FAS II) Pathway ………………............65

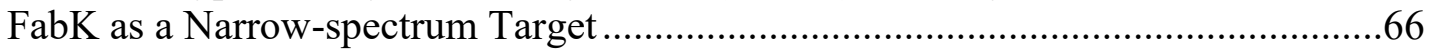

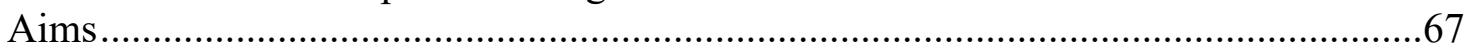

Protein Expression, Purification, and Stabilization of CdFabK .................................67

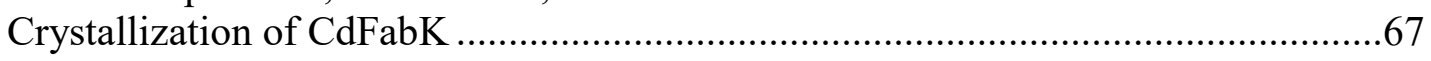

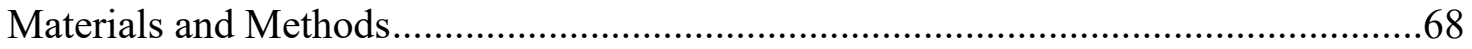

CdFabK Construct Cloning, Plasmid Production, and Expression..............................68

CdFabK_15b and CdFabK_21d Purification........................................................68

CdFabK_21d_NATIVE Purification ..................................................................69

CdFabK Activity and Stability Assays .................................................................69

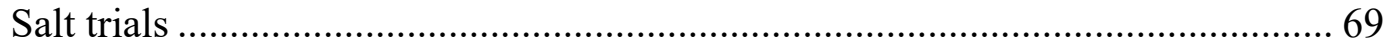




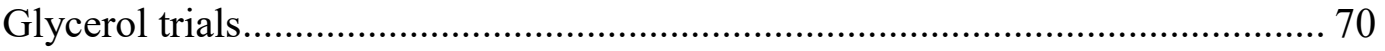

Buffer and $\mathrm{pH}$ trials .................................................................................. 70

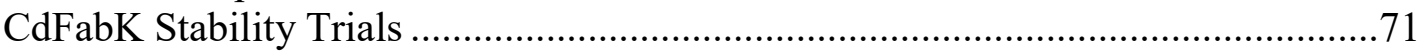

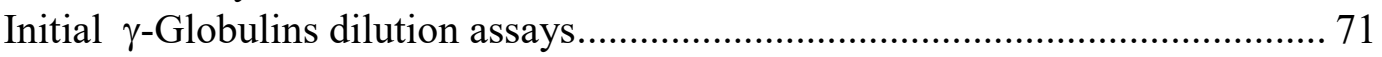

Optimizing $\gamma$-Globulins dilution assays .............................................................. 71

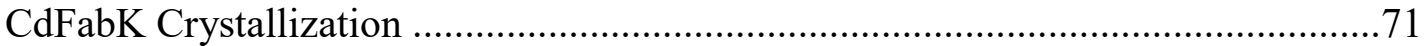

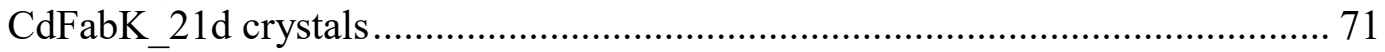

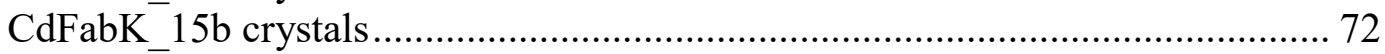

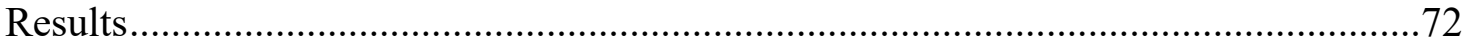

Cloning and Expression of Recombinant CdFabK …………..................................72

Purification of Recombinant CdFabK Constructs ...................................................73

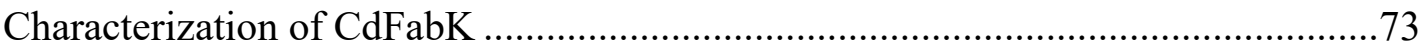

Crystallization of CdFabK_21d and CdFabK_15b................................................77

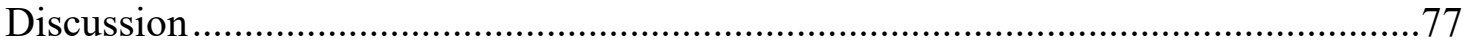

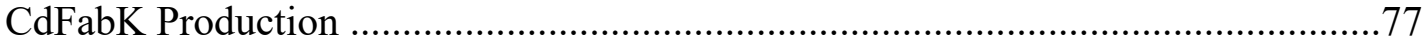

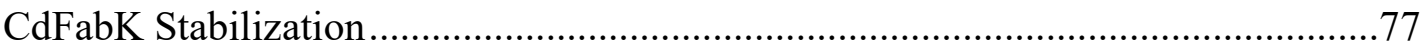

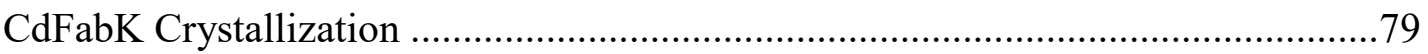

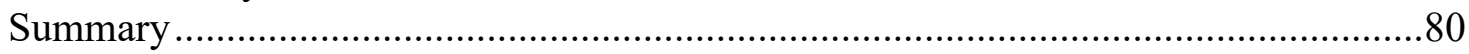

\section{CHAPTER 4. CDFABK ENZYMOLOGY AND MODALITY OF INHIBITION...81}

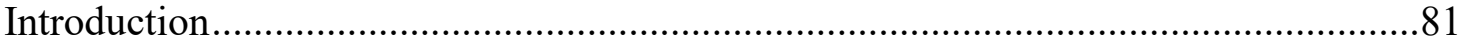

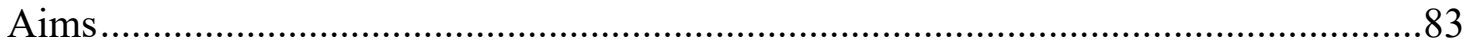

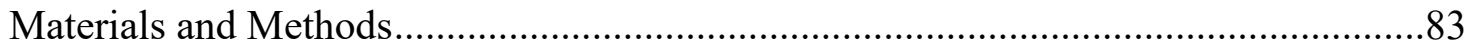

CdFabK_15b Cloning, Expression, Purification.......................................................83

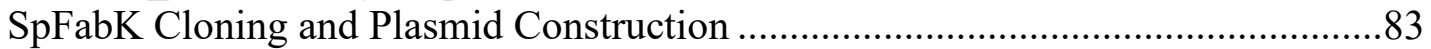

SpFabK_15b Purification.................................................................................... 84

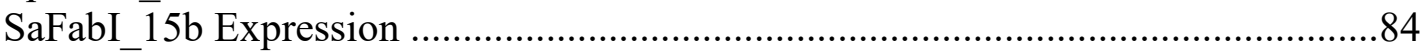

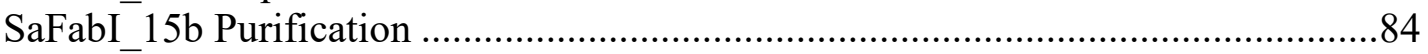

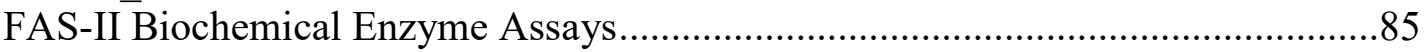

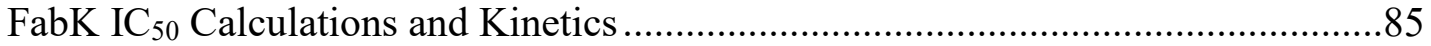

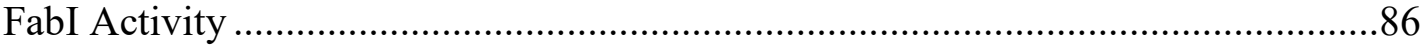

Generation of SpFabK and CdFabK Sequence Alignment........................................86

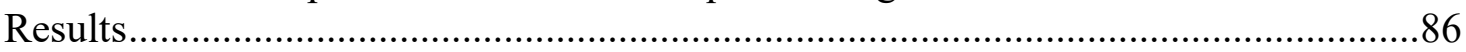

The CdFabK Enzyme Possesses Intrinsic NADH Oxidative Activity and Double

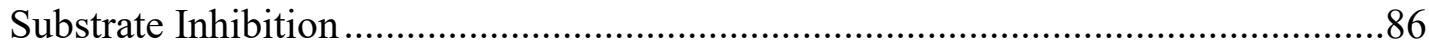

Phenylimidazole-Derived Compounds Selectively Inhibit the $C$. difficile FabK

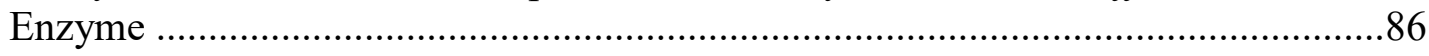

Phenylimidazole Compound $1 \mathrm{~b}$ is Competitive for NADH and Uncompetitive

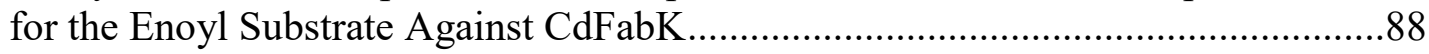

Benzothiazole Compound KH-70 Potently Inhibits CdFabK ....................................90

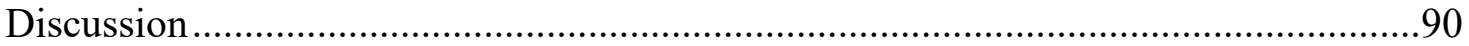

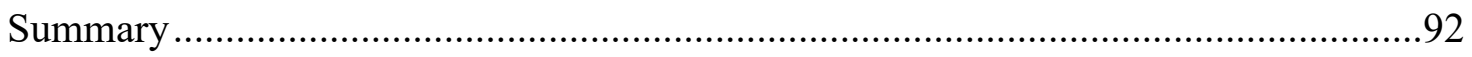

CHAPTER 5. DISCUSSION AND CONCLUSION_..................................................95

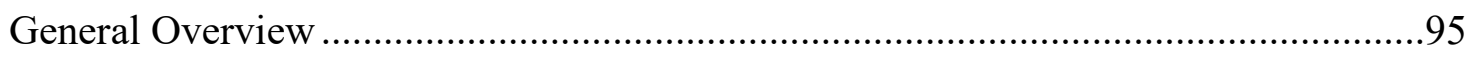

Results and Discussion of Methods and Techniques Employed in This Work .............95 


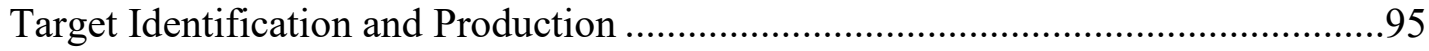

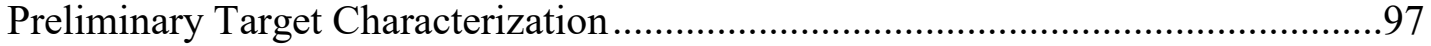

Compound Screening and Hit Selection ................................................................97

Target and Hit Validation and Characterization .....................................................98

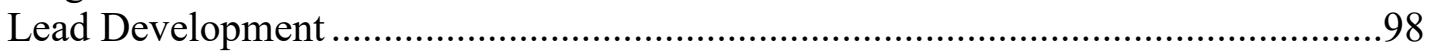

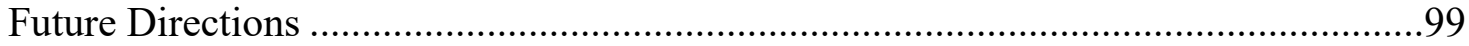

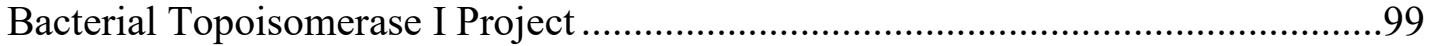

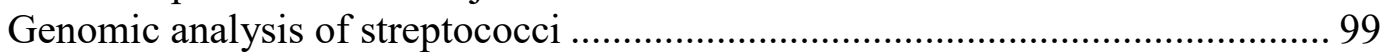

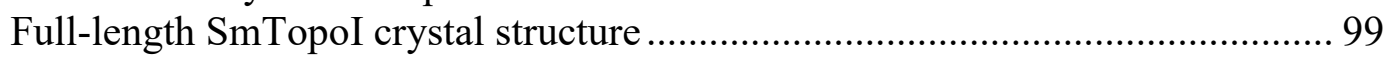

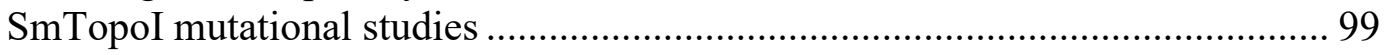

High throughput topoisomerase I assay development .................................. 100

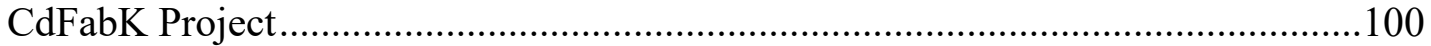

Crystallography ................................................................................... 100

Further hit validation and characterization ................................................... 101

Compound screening ......................................................................... 101

Orthogonal high throughput assay development ......................................... 101

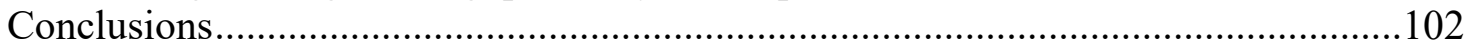

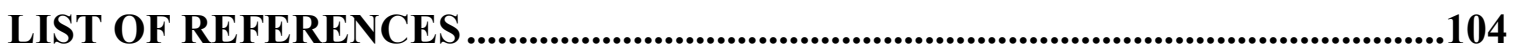

VITA 


\section{LIST OF TABLES}

Table 1-1. Overview of antibacterial agents in clinical trials or recently approved. ......6

Table 2-1. Overview of types and subtypes of select topoisomerases. .........................23

Table 2-2. Topoisomerases present in clinically relevant bacteria. ............................25

Table 2-3. Comparative summary of purification yields from SmTopoI constructs. ....39

Table 2-4. Summary of S. mutans topoisomerase I purification...............................45

Table 2-5. Data collection and refinement statistics for SmTopo_N65......................52

Table 4-1. Inhibitory activity of Enoyl-ACP isozymes by phenylimidazole

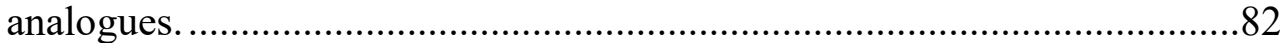




\section{LIST OF FIGURES}

Figure 1-1. Antibacterial eras and recent advances......................................................

Figure 1-2. Drug discovery skills and techniques used in the investigation of narrow spectrum targets in antibacterial drug discovery...........................................13

Figure 2-1. S. mutans topoisomerase I structural homology model. ..............................26

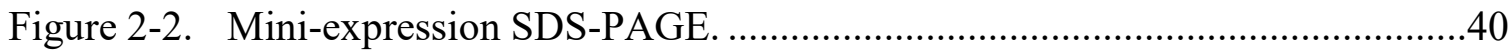

Figure 2-3. Catalase contaminant SDS PAGE. ……………………..........................

Figure 2-4. Chromatogram of nickel column linear elution from preliminary

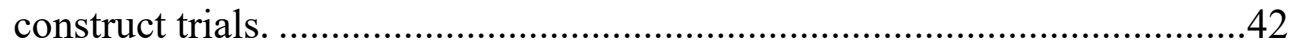

Figure 2-5. SDS-PAGE gels of SmTopI_16b purification results. ..................................44

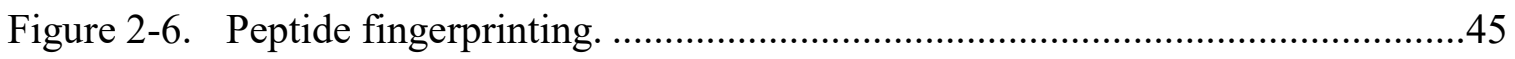

Figure 2-7. Gel-based DNA relaxation assay..............................................................

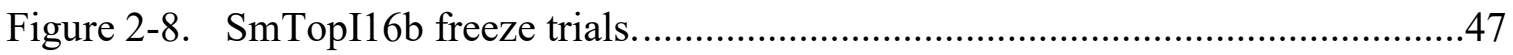

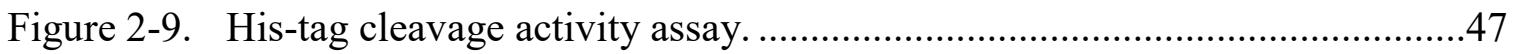

Figure 2-10. Non-Specific DNase Activity Assay. …………………………………......49

Figure 2-11. Thermal stability data. ………………………………………………....50

Figure 2-12 SmTopoI_N65 crystals. .........................................................................

Figure 2-13. Multiple sequence alignment of SmTopoI_N65 and structural homologs. ..53

Figure 2-14. Overall structure of SmTopoI_N65 ………………………………….....54

Figure 2-15. SmTopoI_N65 topology diagram............................................................56

Figure 2-16. Multiple sequence alignment of SmTopoI_N65 and EcTopoIII. ..................57

Figure 2-17. Structural alignment of SmTopoI_N65 and TmTopoI (PDB 2GUI) ............59

Figure 2-18. SmTopoI_N65 multiple sequence alignment with select topo I homologs of undetermined structure ..........................................................................61

Figure 3-1. SDS-PAGE gel of CdFabK_15b purification results...................................74

Figure 3-2. Enzyme activity studies of CdFabK_15b. .................................................74 
Figure $3-3$. Enzyme dilution and stability studies of CdFabK_15b...........................75

Figure 3-4. Extended enzyme dilution and stability studies of CdFabK_15b...............76

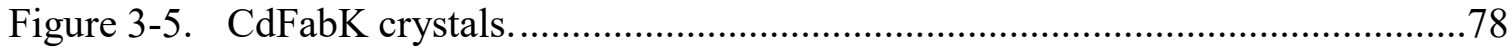

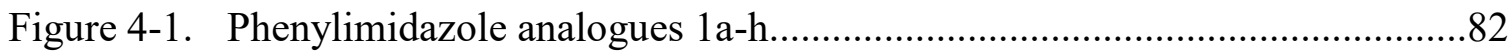

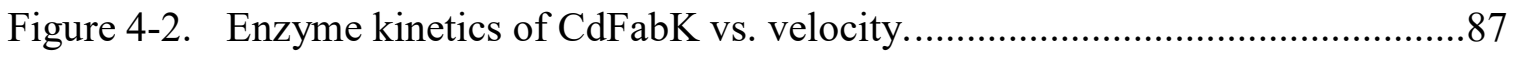

Figure 4-3. Characterization of inhibitory activity and mechanism of $1 \mathrm{~b}$ against CdFabK.

Figure 4-4. Structure and dose response curve against CdFabK for the novel hit KH-

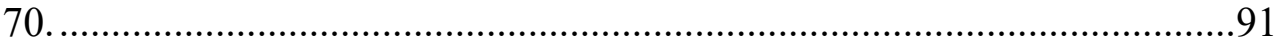

Figure 4-5. Sequence alignment comparing CdFabK and SpFabK .............................93 


\section{LIST OF ABBREVIATIONS}

ACP

BLI

CDI

DHFR

DSF

ESBL

FAS II

FPLC

GAIN

GARDP

GLASS

IACG

IDSA

IMAC

HIC

HTS

IPTG

ITC

LIC

MOA

MDR

MRSA

MS

MSSA

MWCO

$\mathrm{NADH}$

$\mathrm{NADPH}$

NBTI

ND4BB

NDM

NMR

PA

PCR

PDB

QIDP

SAR

SEC

SDR

SDS-PAGE

SOA

SPR

TopoI
Acyl carrier protein

B-lactamase inhibitor

Clostridiodes difficile infection

Dihydrofolate reductase

Differential scanning fluorimetry

Extended-Spectrum $\beta$-Lactamase

Bacterial Type II fatty acid synthesis pathway

Fast protein liquid chromatography

Generating Antibiotics Incentives Now

Global Antibiotic Research and Development Partnership

Global Antimicrobial Resistance Surveillance System

Interagency Coordination Group

Infectious Disease Society of America

Immobilized metal affinity chromatography

Hydrophobic interaction chromatography

High throughput screening

Isopropyl-ß-D-1-thiogalactopyranoside

Isothermal titration calorimetry

Ligation-independent cloning

Mechanism of action

Multi-drug resistant

Methicillin-resistant Staphylococcus aureus

Mass spectrometry

Methicillin-sensitive Staphylococcus aureus

Molecular weight cutoff

Nicotinamide adenine dinucleotide

Nicotinamide adenine dinucleotide phosphate

Novel bacterial topoisomerase inhibitor

New Drugs 4 Bad Bugs

New Delhi metallo- 3 -lactamase

Nuclear magnetic resonance

Pseudomonas aeruginosa

Polymerase chain reaction

Protein Data Bank

Qualified infectious disease product

Structure-activity relationship

Size-exclusion chromatography

Short-chain alcohol dehydrogenase/reductase

Sodium dodecyl sulfate polyacrylamide gel electrophoresis

Spectrum of activity

Surface plasmon resonance

Topoisomerase I 


\section{CHAPTER 1. INTRODUCTION ${ }^{1}$}

\section{Overview}

The current threat of antibacterial resistance looms large across the entire globe. Only a century ago, the threat posed by bacterial infections seemed all but defeated. As initial antibacterial breakthroughs were made, humanity's victory over such infections was generally regarded as a near guarantee. As resistance mechanisms surfaced, however, pathogenic bacterial infections crept their way back into the healthcare arena, regaining an insidious foothold that, in retrospect, now appears to have been underestimated. Resistances continued to evolve, outpacing novel and effective antibacterial drug development and cutting into the hard-won gains humanity had made. Eventually, easily treated infections again became dire and the world is once more on alert, demonstrating the significant need for a modern and concerted effort to develop novel antibacterial agents. A compelling modern argument is being made that such an effort should include, at least in part, an investigation of narrow-spectrum targets for the development of these much needed novel antibacterial agents. A brief survey of the historic eras, modern efforts, and possible future of antibacterial drug discovery may act as a road map for such a case. Furthermore, a discussion of the requirements such narrow spectrum investigations entail helps to accurately present the accompanying benefits and costs.

\section{Antibacterial Drug Discovery Eras: Past, Present, and Future}

\section{The Golden Era}

Before the advent of antibacterial drugs, any insult small or large that introduced the risk of infection was potentially fatal. From a sliver to surgery to simply being in close proximity to a person suffering from a communicable disease, the ever-looming threat of infectious disease was everywhere. However, in the early 1900s, an unprecedented breakthrough occurred. In 1928, Alexander Fleming made his famously "serendipitous" discovery of penicillin, and the "golden era" of antibacterial discovery was born (Figure 1-1). ${ }^{1}$ During this era, phenotypic whole-cell natural product screening predominated, bringing with it a prosperity in antibacterial drug discovery research yet to be rivaled. The majority of antibacterial agents arose from this era, including the penicillins, sulfonamides, vancomycin, streptomycin, chloramphenicol, tetracycline, and erythromycin. In fact, many of these antibiotics, or their derivatives, are still in use and relied upon today.

\footnotetext{
${ }^{1}$ Adapted from final submission by permission of The Royal Society of Chemistry. Jones, J. A., Virga, K. G., Gumina, G. \& Hevener, K. E. Recent Advances in the Rational Design and Optimization of Antibacterial Agents. Medchemcomm 7, 1694-1715, https://doi.org/10.1039/C6MD00232C (2016). ${ }^{1}$
} 


\section{Antibacterial Eras and Recent Advances}

1930s 1940s 1950s 1960s 1970s 1980s 1990s 2000s 2010s

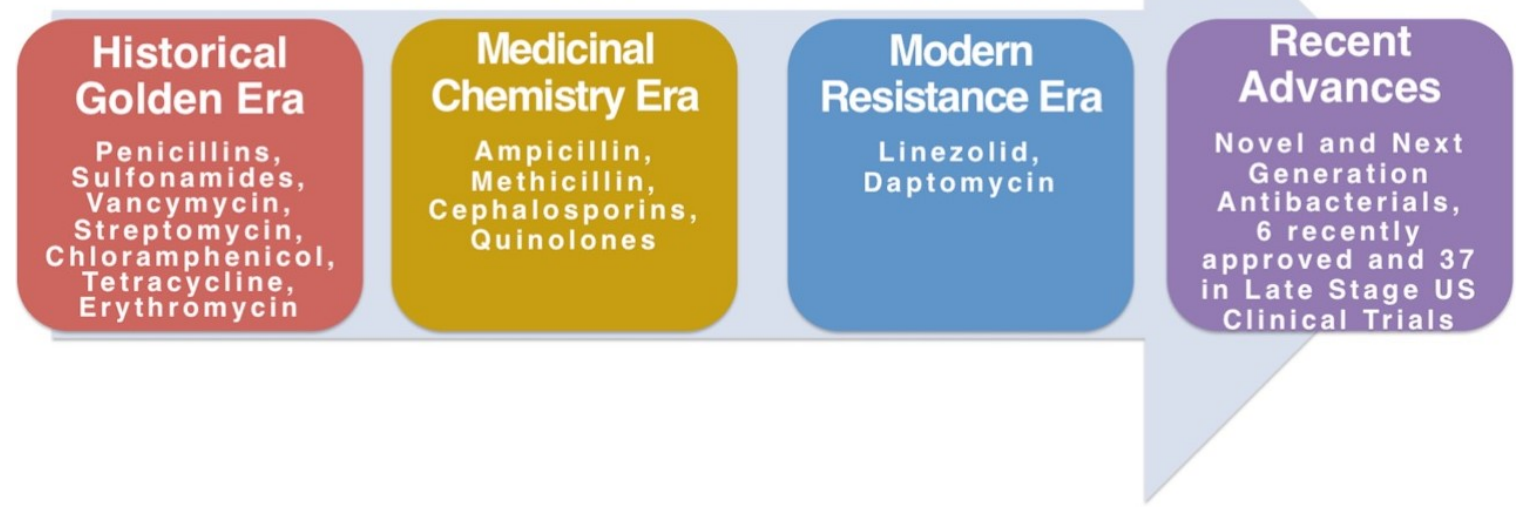

Figure 1-1. Antibacterial eras and recent advances.

Long past the historical "golden era" of antibacterial drug discovery, the modern "resistance era" is being countered by new legislation and advances in the rational design of antibacterial agents.

Reprinted from final submission by permission of The Royal Society of Chemistry. Jones, J. A., Virga, K. G., Gumina, G. \& Hevener, K. E. Recent Advances in the Rational Design and Optimization of Antibacterial Agents. Medchemcomm 7, 1694-1715, https://doi.org/10.1039/C6MD00232C (2016). ${ }^{1}$ 


\section{The Medicinal Chemistry Era}

An effective follow-up to this preeminent era occurred in the mid- to latetwentieth century in the form of the formidable "medicinal chemistry era." Born out of the golden era, the medicinal chemistry era forsook the phenotypic screening modalities that preceded it and instead shifted researchers' focus toward an elegant combination of synthetic modifications of previously discovered compounds, high-throughput screening, and prototypical library design. ${ }^{2}$ Antibacterial agents discovered or developed during this era include ampicillin, methicillin, the cephalosporins, and the quinolones. While early hope and expectations at the time were that this era would usher in a veritable end to the long reign of bacterial infections, history would more realistically highlight it as an incognizant precursor to the modern "resistance era" - an age marked by the menace of expanding bacterial resistance coupled with the unfavorable reality of an inversely diminishing antibacterial pipeline. ${ }^{3}$

\section{The Resistance Era}

In stark comparison to the gains made against bacterial infections during the historical golden and medicinal chemistry eras, the contemporary resistance era is overshadowed by a resurgence of infections for which modern antibacterials are less or altogether ineffective, such as the "ESKAPE" pathogens (Enterococcus faecium, Staphylococcus aureus, Klebsiella pneumoniae, Acinetobacter baumanii, Pseudomonas aeruginosa, and Enterobacteriaceae species). Moreover, the current drug discovery climate is noticeably less fruitful than that of its forerunners, due in part to the fact that it is geared around more arduous and time-consuming rational drug design modalities that highlight target-based high throughput screening (HTS) campaigns while implementing comparatively exhaustive and limited tools like combinatorial libraries. ${ }^{4}$ The few antibacterial agents developed during this era include linezolid and daptomycin. Apart from relatively slower process inherent to rational drug design and discovery, additional issues are associated with contemporary efforts that were, for the most part, innately avoided in the past by the very nature of the phenotypic whole-cell screening methods used in the golden era include difficulties with inadequate cell penetration, drug efflux, and in vivo instability, to name a few. Overall, the relatively reduced output of the modern drug discovery era combined with the accelerating occurrence of antibacterial drug resistance and the rapid rate at which infections spread with modern travel has merged to form an alarming modern public health concern with an even more concerning prognosis of the future.

\section{The Post-Antibiotic Era}

Looking ahead on the antibacterial timeline, the next major antibacterial drug discovery era is commonly forecasted as the "post-antibiotic era," which foretells the existence of a bleak period reminiscent of the time before the existence of antibacterial agents - a future in which human deaths attributed to resistant microorganisms rises from the current 700,000 per year to an excess of 10 million per year. ${ }^{2}$ The United Nations (UN) World Health Organization (WHO) Ad hoc Interagency Coordination Group on 
Antimicrobial Resistance (IACG) reported this year (2019) that by 2050, drug-resistant microbial diseases could cause economic damage on par to the 2008-2009 global financial crisis, and that by 2030, up to 24 million people could be forced into extreme poverty due simply to the effects of antimicrobial resistance. ${ }^{5}$ In order to adequately combat this threat, a multi-faceted approach has commenced. Both public and private entities across the globe have risen to meet the challenge of antimicrobial resistance in an effort to avert the post-antibiotic era, including new legislative and research efforts.

\section{Public and Private Responses to the Threat of Antimicrobial Resistance}

Within the last several years of the "resistance era," the United States has launched several initiatives aimed at curbing the threat of infectious diseases including legislation like the Generating Antibiotics Incentives Now Act (GAIN Act) aimed at fasttracking the development and approval of Qualified Infectious Disease Products (QIDPs). Likewise, Europe has recently implemented its own similar responses, such as the Innovative Medicines Initiative (IMI) ENABLE project under the "New Drugs 4 Bad Bugs" (ND4BB) program aimed specifically at battling Gram-negative bacteria. The WHO has also led multiple initiatives aimed at addressing antibacterial awareness, including the launch of World Antibiotic Awareness Week, the Global Antimicrobial Resistance Surveillance System (GLASS), the Global Antibiotic Research and Development Partnership (GARDP), as well as the formation of the aforementioned IACG. However, while momentum has increased, these governmental responses did not manifest themselves spontaneously. In large part, they were the result of long-fought battles by private citizens and associations working diligently to assess, interpret, and raise the alarm regarding the overall problem - parts of an overall war still being fought.

Appropriately combatting the modern threat of microbial disease centers around countering antibacterial resistance. Therefore, it is arguable that the individuals best suited to assess the threat are infectious disease-focused healthcare professionals themselves. Indeed, an early leader in this fight, the Infectious Disease Society of America (IDSA) has gone to great lengths to increase public awareness of the looming problem. As early as 2002, IDSA publicly conveyed worry for the lack of noteworthy research and development (R\&D) aimed at combatting multidrug resistant (MDR) microbial infections. ${ }^{6}$ By 2004, IDSA had issued a clarion call to government agencies for the enactment of formal regulatory and funding initiatives to aid in the fight against the forthcoming antibacterial resistance crisis. ${ }^{7}$ Moreover, in 2010, IDSA introduced its "10 x "20 Initiative" calling for R\&D sufficient to bring 10 novel and effective systemic antibacterial agents by the year $2020 .^{8}$ By 2013 , an update by the IDSA communicated some advancement had been made, but that it still appeared inadequate to properly confront the growing problem of resistance. ${ }^{9}$

That same year, the Centers for Disease Control and Prevention (CDC) appeared to take the regulatory lead when it released its report titled "Antibiotic resistance threats in the United States, 2013." 10 In the report, the CDC called out four "core actions" to prevent antibiotic resistance, which included preventing the spread of infections and, 
therefore, the spread of resistance; tracking antibiotic resistant infections; improving antibiotic prescribing via effective stewardship; and lastly, developing new diagnostic tests and new drugs. Because the natural evolution of bacteria includes antibiotic resistance, the CDC notes, the threat can be slowed with adequate measures, but never completely avoided or suspended entirely. This concept has been showcased recently by the existence and conservation of various elements of bacterial resistomes from isolated cave bacteria previously unexposed to modern antibacterial drugs. ${ }^{11}$ Because of this sobering fact, it is expected that a perpetual need for novel antibacterial drugs will always exist.

\section{Recent Advances in Antibacterial Drug Discovery, the Modern Era}

Attempts to address the modern challenge of antibacterial drug discovery have taken place via several different avenues. Since the 1980s, FDA approvals of new antibacterial agents steadily declined from sixteen approvals between 1983 and 1987 to only two approvals between 2011 and 2012, then rebounded between 2013 and 2017, increasing to seven approvals during that period. ${ }^{6}$ First glance suggests that private and public initiatives have begun to have a positive effect, resulting in a recent upswing in antibacterial drug discovery efforts. However, with the exception of an extremely small number of antibacterial agents possessing a novel mechanism of action (MOA), modern research efforts have primarily focused on the optimization of known antibacterial agents emphasizing rational design strategies geared around classic, conventional targets ${ }^{1,6}$ (Table 1-1). For the most part, the substantial bulk of these targets are well validated and have a long history of practical exploitation. ${ }^{4}$ In general, these modern antibacterial efforts have revolved around improving or regaining overall spectra of activity of the agents, as well as improving physicochemical properties, pharmacokinetics, adverse effects, off-site activities, and so on. In these efforts, modern antibacterial researchers have recently begun to "re-discover" natural products chemistry, complementing their endeavors with contemporary approaches like rational semi-synthetic modifications. ${ }^{12,13}$

Recent advances in the rational design and optimization of antibacterial agents include the development of various agents across multiple drug classes. Developmental status of these novel agents range from early-stage clinical trials to recent approval by the U.S. Food and Drug Administration (FDA) while, unfortunately, some hopeful products have recently been withdrawn altogether. ${ }^{1}$ A brief summary of recently developed (within the last decade), mostly small molecule antibacterial agents is as follows:

\section{Advanced generation cephalosporins}

Recent advances in the chemistry of the $\beta$-lactam antibiotics have resulted in the approval and late-stage development of several advanced (or $5^{\text {th }}$ ) generation cephalosporins that boast enhanced spectra of activity (SOA), including activity against some Extended-Spectrum B-Lactamase (ESBL) producers, methicillin-resistant Staphylococcus aureus (MRSA), and Pseudomonas aeruginosa (PA). There are currently 
Table 1-1. Overview of antibacterial agents in clinical trials or recently approved.

\begin{tabular}{|c|c|c|c|c|c|c|}
\hline \multirow[b]{2}{*}{ Class } & \multirow[b]{2}{*}{$\begin{array}{c}\text { Novel } \\
\text { Agents* }\end{array}$} & \multirow[b]{2}{*}{$\begin{array}{l}\text { Novel } \\
\text { MOAs }\end{array}$} & \multirow[b]{2}{*}{$\begin{array}{c}\text { Novel } \\
\text { Targets }\end{array}$} & \multicolumn{3}{|c|}{ Status } \\
\hline & & & & $\begin{array}{c}\text { Early Stage } \\
\text { (Phase 1) }\end{array}$ & $\begin{array}{l}\text { Late Stages } \\
\text { (Phase } 2 \text { to 3) }\end{array}$ & $\begin{array}{l}\text { Recently } \\
\text { Approved }\end{array}$ \\
\hline Advanced Generation Cephalosporins & 4 & 1 & 0 & $\mathbf{0}$ & 2 & 2 \\
\hline Cefiderocol & & No & No & & Phase 3 & \\
\hline Ceftaroline & & No & No & & & 2010 \\
\hline Ceftabiprole & & YES & No & & Phase 3 & \\
\hline Ceftolozane & & No & No & & & 2014 \\
\hline Next-Generation Beta-Lactamase Inhibitors & 8 & $\mathbf{0}$ & $\mathbf{0}$ & 4 & 2 & 2 \\
\hline AI101 & & No & No & & Phase 3 & \\
\hline Avibactam & & No & No & & & 2015 \\
\hline ETX2514 & & No & No & Phase 1 & & \\
\hline Nacubactam & & No & No & Phase 1 & & \\
\hline Relebactam & & No & No & & Phase 3 & \\
\hline Vaborbactam & & No & No & & & 2017 \\
\hline VNRX-5133 & & No & No & Phase 1 & & \\
\hline Zidebactam & & No & No & Phase 1 & & \\
\hline Next-Generation Oxazolidinones & 3 & $\mathbf{0}$ & $\mathbf{0}$ & 0 & 2 & 1 \\
\hline Contezolid (MRX-I) & & No & No & & Phase 2 & \\
\hline Radezolid & & No & No & & Phase 2 & \\
\hline Tedizolid & & No & No & & & 2014 \\
\hline Next-Generation Bacterial Topoisomerase Inhibitors & 7 & 2 & $\mathbf{0}$ & $\mathbf{0}$ & 5 & 2 \\
\hline Avarofloxacin & & No & No & & Phase 2 & \\
\hline Delafloxacin & & No & No & & & 2017 \\
\hline Finafloxacin & & No & No & & & 2014 \\
\hline Gepotidacin & & YES & No & & Phase 2 & \\
\hline Nemonoxacin & & No & No & & Phase 3 & \\
\hline Zabofloxacin & & No & No & & Phase 3 & \\
\hline Zoliflodacin & & YES & No & & Phase 2 & \\
\hline Next-Generation Tetracyclines & 3 & 0 & $\mathbf{0}$ & 1 & 0 & 2 \\
\hline Eravacycline approved 2018 & & No & No & & & 2018 \\
\hline Omadacycline approved 2018 & & No & No & & & 2018 \\
\hline TP6076 Phase 1 & & No & No & Phase 1 & & \\
\hline Next-Generation Macrolides & 3 & $\mathbf{0}$ & $\mathbf{0}$ & $\mathbf{0}$ & 3 & $\mathbf{0}$ \\
\hline Solithromycin & & No & No & & Phase 3 & \\
\hline Nafithromycin & & No & No & & Phase 2 & \\
\hline Cethromycin & & No & No & & Phase 3 & \\
\hline
\end{tabular}


Table 1-1. Continued.

\begin{tabular}{|c|c|c|c|c|c|c|}
\hline \multirow[b]{2}{*}{ Class } & \multirow[b]{2}{*}{$\begin{array}{c}\text { Novel } \\
\text { Agents* }\end{array}$} & \multirow[b]{2}{*}{$\begin{array}{l}\text { Novel } \\
\text { MOAs }\end{array}$} & \multirow[b]{2}{*}{$\begin{array}{l}\text { Novel } \\
\text { Targets }\end{array}$} & \multicolumn{3}{|c|}{ Status } \\
\hline & & & & $\begin{array}{l}\text { Early Stage } \\
\text { (Phase 1) }\end{array}$ & $\begin{array}{l}\text { Late Stages } \\
\text { (Phase } 2 \text { to 3) }\end{array}$ & $\begin{array}{c}\text { Recently } \\
\text { Approved }\end{array}$ \\
\hline $\begin{array}{l}\text { Next-Generation Aminoglycosides } \\
\text { Plazomicin }\end{array}$ & 1 & $\begin{array}{c}\mathbf{0} \\
\text { No }\end{array}$ & $\begin{array}{c}\mathbf{0} \\
\text { No }\end{array}$ & $\mathbf{0}$ & $\mathbf{0}$ & $\begin{array}{c}1 \\
2018\end{array}$ \\
\hline $\begin{array}{l}\text { Novel Bacterial Folate Synthesis Inhibitors } \\
\text { Iclaprim }\end{array}$ & 1 & $\begin{array}{c}\mathbf{0} \\
\text { No }\end{array}$ & $\begin{array}{c}\mathbf{0} \\
\text { No }\end{array}$ & $\mathbf{0}$ & $\begin{array}{l}1 \\
\text { NDA }\end{array}$ & $\mathbf{0}$ \\
\hline $\begin{array}{l}\text { Novel Inhibitors of the Bacterial FAS-II Pathway } \\
\text { Afabicin desphosphono (Debio-1452) } \\
\text { CG400549 }\end{array}$ & 2 & $\begin{array}{l}1 \\
\text { YES } \\
\text { No }\end{array}$ & $\mathbf{0}$ & $\mathbf{0}$ & $\begin{array}{c}2 \\
\text { Phase } 2 \\
\text { Phase } 2\end{array}$ & $\mathbf{0}$ \\
\hline Novel Lipoglycopeptides & 2 & $\mathbf{0}$ & $\mathbf{0}$ & $\mathbf{0}$ & 0 & 2 \\
\hline Dalbavancin & & No & No & & & 2014 \\
\hline Oritavancin & & No & No & & & 2014 \\
\hline $\begin{array}{l}\text { Novel Penems } \\
\text { Sulopenem Phase } 3 \\
\text { Tebipenem (SPR994) Phase } 1\end{array}$ & 2 & $\begin{array}{c}\mathbf{0} \\
\text { No } \\
\text { No }\end{array}$ & $\begin{array}{c}\text { 0 } \\
\text { No } \\
\text { No }\end{array}$ & $\begin{array}{c}1 \\
\text { Phase } 1\end{array}$ & $\begin{array}{c}1 \\
\text { Phase } 3\end{array}$ & 0 \\
\hline $\begin{array}{l}\text { Novel Monobactams } \\
\quad \text { LYS228 }\end{array}$ & 1 & $\begin{array}{c}\mathbf{0} \\
\text { No }\end{array}$ & $\begin{array}{c}\mathbf{0} \\
\text { No }\end{array}$ & 0 & $\begin{array}{c}1 \\
\text { Phase } 2\end{array}$ & $\mathbf{0}$ \\
\hline $\begin{array}{l}\text { Other Novel Agents } \\
\text { Fosfomycin disodium (epoxide, cell wall synthesis inhibitor) } \\
\text { Fusidic acid (fusidane, protein synthesis inhibitor) } \\
\text { Lefamulin (pleuromutulin, protein synthesis inhibitor) } \\
\text { Murepavadin (POL7080; peptide mimetic, LPS inhibitor) } \\
\text { SPR741 partner antibiotic (cationic potentiator) }\end{array}$ & 5 & $\begin{array}{l}1 \\
\text { No } \\
\text { No } \\
\text { No } \\
\text { YES } \\
\text { No }\end{array}$ & $\begin{array}{l}1 \\
\text { No } \\
\text { No } \\
\text { No } \\
\text { YES } \\
\text { No }\end{array}$ & $\begin{array}{c}1 \\
\text { Phase } 1\end{array}$ & $\begin{array}{l}\text { 4 } \\
\text { NDA } \\
\text { Phase } 3 \\
\text { NDA } \\
\text { Phase } 3\end{array}$ & $\mathbf{0}$ \\
\hline TOTAL & 42 & 5 & 1 & 7 & 21 & 12 \\
\hline
\end{tabular}

Does not include "novel" combination products comprised of multiple non-novel agents.

DHFR, dihydrofolate reductase; LPS, lipopolysaccharide; MOA, mechanism of action; NDA, new drug application; FAS-II, bacterial fatty acid biosynthesis pathway.

Data sources: Jones, J. A., Virga, K. G., Gumina, G. \& Hevener, K. E. Recent Advances in the Rational Design and Optimization of Antibacterial Agents. Medchemcomm 7, 1694-1715, https://doi.org/10.1039/C6MD00232C (2016). ${ }^{1}$

Talbot, G. H. et al. The Infectious Diseases Society of America's 10 x '20 Initiative (Ten New Systemic Antibacterial Agents FDAapproved by 2020): Is 20 x '20 a Possibility? Clin Infect Dis, https://doi.org/10.1093/cid/ciz089 (2019). ${ }^{6}$ 
two next generation cephalosporins that were recently approved and two in late-stage development (Phase 2 or Phase 3) in the U.S. ${ }^{6}$

\section{Next generation beta lactamase inhibitors}

The development of two new classes of $\beta$-lactamase inhibitors (BLIs) has resulted in a much-needed respite from the steadily increasing threat of multi-drug resistant (MDR) and pan-drug resistant bacterial infections. There are currently eight novel BLIs of note, with four recently approved or in late-stage development, and four more in early stage development (Phase 1). ${ }^{6}$

\section{Next generation oxazolidinones}

The oxazolidinone class of antibacterials is a fully synthetic class of agents and has been considered a major breakthrough in antimicrobial drug development with linezolid standing as the best-known representative. ${ }^{14}$ The recent emergence of linezolid resistance has driven the investigation and advancement of next-generation oxazolidinones with improved antibacterial activities and decreased adverse effect profiles. ${ }^{15-17}$ To that aim, one novel oxazolidinone has recently been approved, and two more are in late-stage development. ${ }^{6}$

\section{Next generation bacterial topoisomerase inhibitors}

Bacterial topoisomerases are extremely well validated as efficacious antibacterial drug targets, and the type II topoisomerase inhibitors known as quinolone antibiotics have proven to be one of the most practically effective antibacterial classes in recent history. Quinolones have shown astonishing popularity over the last several decades, perhaps contributing to the sharp rise in use, or overuse, and subsequent respective

resistance rates across the country. ${ }^{18}$ Nonetheless, bacterial topoisomerases have gained a great deal of attention within the realm of antibacterial drug development, and several inhibitors within this class have either been recently approved or are in late-stage clinical trials. Additionally, this is one of the few classic targets with new antibacterial agents being developed that showcase novel MOAs. One of these classes of new agents, known as novel bacterial topoisomerase inhibitors (NBTIs), consequently lack cross-resistance among pathogens possessing quinolone resistance. Additionally, the newer spiropyrimidinetrione class has recently arisen with yet another unique mechanism of topoisomerase II inhibition. Though discovered via whole-cell activity screens, spiropyrimidinetriones have since been rationally optimized. ${ }^{19}$ As one would expect, the unique MOIs of these newer classes imply accordingly unique structures, scaffolds, and SARs. Overall, two novel bacterial topoisomerase inhibitors have recently been approved, and five are in late-stage development. ${ }^{6}$

\section{Next generation tetracyclines}

Tigecycline (Tygacil@; Wyeth Pharmaceuticals Inc., a subsidiary of Pfizer Inc., initial U.S. approval in 2005) is the first semi-synthetic representative of a class of 
antimicrobials called glycylcyclines. ${ }^{20}$ The design and synthesis of glycylcyclines were intended to overcome the major problems of modern ribosomal protection and effluxpump-mediated tetracycline resistance. ${ }^{21,22}$ The success and utility of tigecycline, coupled with climbing resistance rates against other major antibacterial classes, have recently prompted the development of several more novel tetracycline analogues, including two recently approved agents and one in early stage development. ${ }^{6}$

\section{Next generation macrolides}

Macrolide antibiotics are natural product-based antibacterials composed of a large, macrocyclic lactone core, to which a variety of sugar substituents may be attached. Disadvantages of the macrolide antibiotics include limited Gram-negative activity, acid instability limiting the oral effectiveness of some compounds in the class, and interaction with human drug metabolizing enzymes in the cytochrome P-450 system that can result in significant drug interactions. ${ }^{23}$ Further, emerging resistance, typically due to alterations of the macrolide binding site on the ribosome, has limited the clinical utility of older compounds in the class and, therefore, novel modified ketolide antibacterials have been developed to overcome such issues. To date, three novel macrolides are currently in late-stage development.

\section{Next generation aminoglycosides}

Development of next-generation aminoglycosides has been somewhat limited. Nonetheless, semi-synthetic techniques have been implemented to develop a single novel aminoglycoside, plazomicin. It has shown activity against a broad range of Gram-positive and Gram-negative organisms, including ESBL-producing pathogens, when used in combination therapy with other antibacterial agents. ${ }^{24}$ Plazomicin is not active, however, against organisms harboring aminoglycoside resistance due to the expression of ribosomal methylases. ${ }^{6,25}$ It is the sole next-generation aminoglycoside in development, and was very recently approved. ${ }^{6}$

\section{Novel bacterial folate synthesis inhibitor}

Iclaprim (Motif Bio, PLC) is the sole next generation dihydrofolate reductase (DHFR) inhibitor in late-stage development. It was rationally designed using structural data and molecular modeling, and has potent Gram-positive bactericidal activity that includes activity against methicillin-sensitive $S$. aureus (MSSA), MRSA, and trimethoprim-resistant F98Y mutant strains. ${ }^{26,27}$ Activity against Gram-negative and atypical organisms is reported to be similar to that of trimethoprim. ${ }^{28}$ Iclaprim was granted qualified infectious disease product (QIDP) status and is in the NDA stage. ${ }^{6}$

\section{Novel inhibitors of the bacterial FAS-II pathway}

Two promising anti-staphylococcal FabI inhibitors are currently under development, both of which represent notable rational design methods. The first compound, CG400549, was designed to overcome the unfavorable pharmacokinetics of 
triclosan by replacing a metabolically unstable phenol group with a pyridone ring system and the small ether linker with a methylene group. ${ }^{29,30}$ CG400549 binds to the FabINADPH (reduced cofactor) binary complex, making the drug uncompetitive with respect to NADPH binding and competitive with respect to the binding of the FabI enoyl substrate. The other FabI inhibitor in development, afabicin (Debio-1450) was developed using a classically iterative, structure-guided strategy, and showcases a unique MOA where the agent binds to a binary complex of the enzyme and its oxidized cofactor, $\mathrm{NADP}^{+}$. 31-33 Modern structural information has guided recent efforts to expand the activity of CG400549 by iterative, structure-guided design strategies, to include Gramnegative pathogens and mycobacteria. ${ }^{29}$ Both GC400549 and afabicin are in late-stage development. ${ }^{6}$

\section{Novel lipoglycopeptides}

The lipoglycopeptides contain large lipophilic moieties linked to glycopeptides, making their molecular weight notably high and, therefore, inherently separate from "small molecule" antibacterials. ${ }^{34}$ Nonetheless, two lipoglycopeptides with notable Gram-positive activity, dalbavancin and oritavancin, were approved within the last five years. ${ }^{6}$

\section{Novel penems}

The carbapenems are $\beta$-lactam antibacterials developed several decades ago that showed exceptional activity against MDR bacteria. ${ }^{35}$ Their use has become more limited over the years, though, as resistance to the class was observed very soon after their clinical emergence. ${ }^{36}$ Recent development of novel penems has resulted in one agent in early stage development and one oral agent in late-stage development. ${ }^{6}$

\section{Novel monobactam}

Monobactams were discovered several decades ago, with the prototype aztreonam showcasing, as the class name would indicate, an unfused B-lactam ring. ${ }^{37}$ While aztreonam is susceptible to serine beta lactamases, the novel monobactam LYS228 shows greater stability against SBLs and is currently in late-stage development.

\section{Other novel agents}

While more difficult to classify, several other novel antibacterial agenta are currently under development. These include the cell wall synthesis inhibitor fosfomycin, currently undergoing NDA status; the protein synthesis inhibitor fusidane, known as fusidic acid, currently in late-stage development; the pleuromutlin protein synthesis inhibitor known as lefamulin, currently in NDA status; the cationic, Gram-negative outer membrane targeting potentiator known as SPR741, currently in early-stage development; and Murepavadin (POL7080, Polyphor, Ltd.), which is a synthetic beta hairpin peptidomimetic with anti-pseudomonal activity currently in late-stage development and is, notably, the only novel antibacterial agent with a truly novel target. ${ }^{6,38}$ It is worth 
mentioning, however, that as a peptide, this agent is not a small molecule antibacterial, per se.

\section{The Narrow Spectrum Era}

Current techniques and methods being implemented in the rational design and optimization of antibacterial agents suggest a subtle change in the recent state of affairs regarding the struggle against bacterial infections. As described above, recent advances in various techniques have helped gain small amounts of ground in the modern struggle against resistant bacterial infections - a conflict where prolonged trends have mostly demonstrated losses. Promising standouts include specific chiral configurations of new quinolones that grant improved activity under acidic conditions, fully-synthetic techniques used in the next-generation tetracyclines that allow for novel side-chains that grant activity against resistant pathogens, and semi-synthetic techniques used in the nextgeneration macrolides that allow for the modification of functional groups that improve binding and, again, activity against resistant pathogens. The recent advances in the rational design and optimization of antibacterial agents are both encouraging in the fact that the downward trend in FDA approvals has been, if not reversed, at least momentarily slowed. Nonetheless, results are also somewhat discouraging in the fact that up until this point, all recent approvals lack novel targets.

With newly implemented incentivization and modern advances in research methods, it is hoped that the threat of antibacterial resistance will continue to be met with appropriate countermeasures. The number of novel antibacterial agents that were recently approved or under late-stage development is highly encouraging, as is recent U.S. and European legislation facilitating the rapid development and approval of new antibacterials. By 2016 alone, there were almost 60 antimicrobial drugs granted QIDP status in the U.S., six of which had already attained U.S. approval, and 37 that were in late-phase clinical trials. In the last ten years, twelve new antibacterial agents were approved and, by 2019 , three agents were in NDA status. ${ }^{6}$ These results stand as evidence for the initial success of these new initiatives and their ability to drive the advancement of antibacterial development. As mentioned above, however, a thorough analysis of the antibacterial pipeline showcases a trend that is still somewhat troubling. While there has been a noteworthy increase in antibacterial drug discovery and development, the astonishingly low number of compounds under development with original targets highlights the urgent need for the characterization and validation of such.

This need for novel, validated targets has been emphasized by numerous reports from public health agencies across the world indicating the fact that resistance to antibacterial agents that exploit traditional targets continues to surge. Just within the last several years, the CDC reported a fatality within the United States resulting from a strain of New Delhi metallo-ß-lactamase (NDM) possessing Klebsiella pneumoniae found to be resistant to 26 antibiotics, including every aminoglycoside and polymyxin agent tested, and possessing what is assumed to be a novel resistance mechanism to the commonly regarded "antibiotic of last resort," colistin. ${ }^{39}$ Despite encouraging progress being made 
with optimization strategies, relying on classic antibacterial targets alone is simply not enough. It has been argued that ample attention should be turned toward the future development of selective antibacterial targets, a move that is expected to bolster the antibacterial pipeline as well as slow the development of antibacterial resistance. It is reasonable to believe that antibacterial agents geared toward such selective targets would have substantial additional benefits - such as the possibility of developing effective microbiome-sparing agents and the requirement for rational, more responsible use of antibacterial agents that would in turn contribute to greater antibacterial stewardship. This is giving way to what some predict will be the next phase of antibacterial discovery and development- the "narrow spectrum-era." While indeed an exciting prospect, characterizing and validating novel narrow-spectrum targets would of course require an understandably substantial amount of intellectual resources, innovation, and effort previously unseen - including fully rational drug design based around heretofore novel and unconventional targets, the employment of a broad arsenal of drug discovery skills and techniques (Figure 1-2), and a certain degree of anticipated success.

\section{Requisite Antibacterial Drug Discovery Skills and Techniques}

\section{Target identification and production techniques}

Techniques involved with preliminary target identification include a thorough literature review in order to survey the successes, failures, difficulties, and considerations of researchers who may have already pursued similar research paths; genomic bioinformatics techniques for data mining the existence of genes of interest for rational targets in pathogens of interest, as well as comparative genomic techniques for assessing the absence or existence of respective selective or otherwise rational targets in other bacteria; other bioinformatic techniques that allow one to assess certain parameters of the target that will later prove invaluable during target production, characterization, and compound screening - parameters such as target size, stability, isoelectric points, extinction coefficient, solubility, and lipophilicity.

Once a satisfactory rational target has been selected that confers a particular degree of anticipated success, techniques centered around target production become of immediate importance. First, cloning techniques are required to produce the gene of interest that codes for the particular target enzyme. First, the gene must be acquired, must commonly done by polymerase chain reaction (PCR) amplification. Different cloning techniques must then be used to transfer the gene of interest into a suitable expression vector. Such cloning techniques include classic restriction enzyme cloning, TOPO cloning, TA cloning, Gibson cloning, Gateway cloning, ligation-independent cloning (LIC), and others. Decisions regarding codon optimization for anticipated expression organisms must also be considered at this point. Alternatively, the gene and an appropriate vector may also simply be purchased. As with all research, a cost analysis of time, money, manpower, and perhaps learning opportunity must be taken into account when selecting the most suitable technique. Additionally, decisions on later expression 


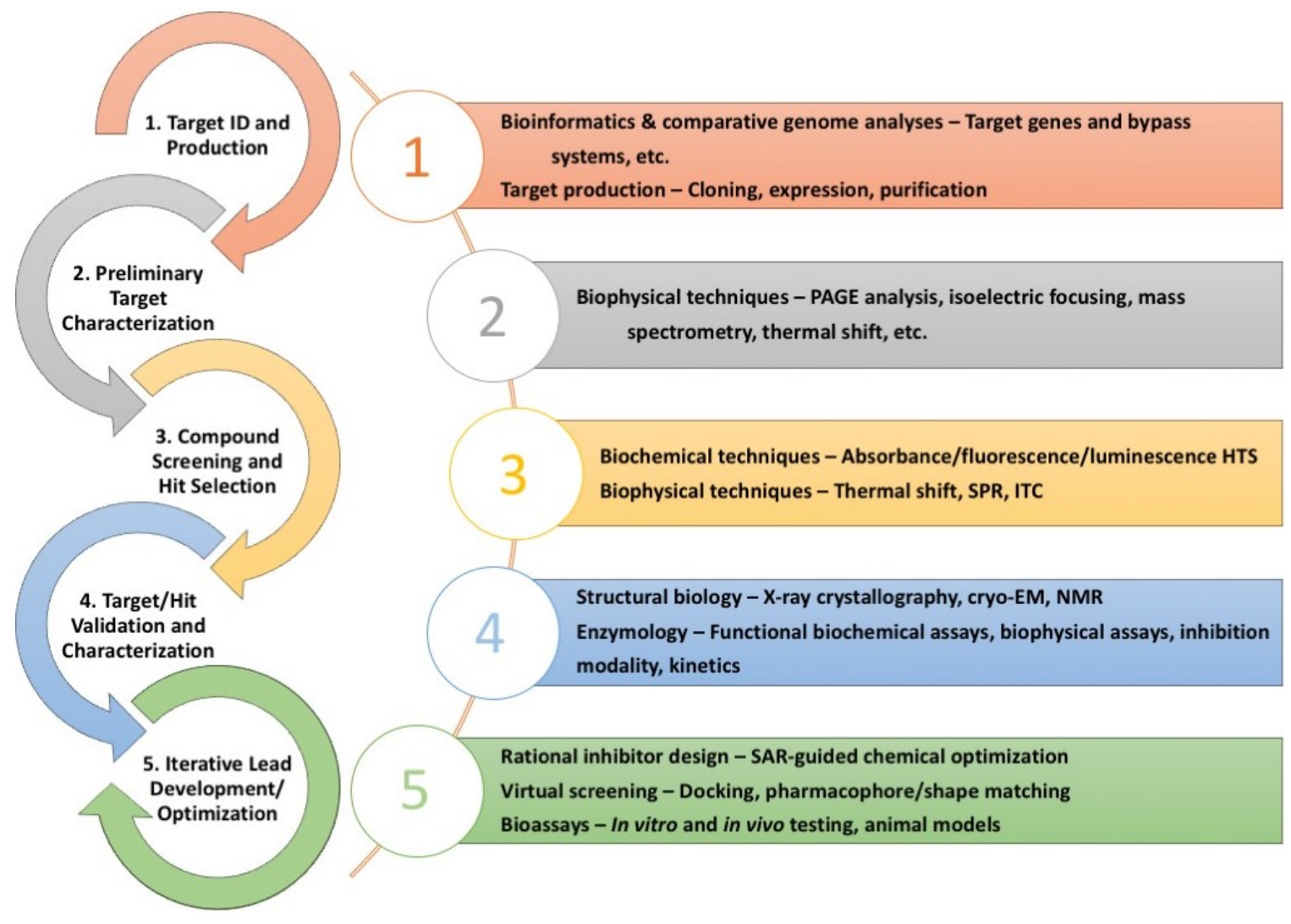

Figure 1-2. Drug discovery skills and techniques used in the investigation of narrow spectrum targets in antibacterial drug discovery. 
and purification techniques must be considered here, as an appropriate vector must be selected that carries the appropriate induction methods to produce the target enzyme, as well as the appropriate tags desired for solubility, recognition, purification, etc. Once the gene of interest is produced and has been inserted into a satisfactory vector, it must be transformed into a production host, such as bacteria (most commonly Escherichia coli), yeast, insect, or mammalian cell lines.

Next, protein production techniques become imminently necessary. This includes growth and induction techniques, such as classic isopropyl-ß-D-1-thiogalactopyranoside (IPTG) induction, tetracycline induction, or autoinduction, among others. Target protein can then be harvested and purified via a number of various popular techniques, ammonium sulfate precipitation, immobilized metal affinity chromatography (IMAC), ion exchange chromatography, hydrophobic interaction chromatography (HIC), and sizeexclusion chromatography (SEC) among some of the most popular. Numerous other techniques are available, including more costly, but also highly specific antibody directed purification systems. A researcher can also use resins and slurries that apply his or her purification modality of choice under gravity filtration, or choose pre-packed columns and fast protein liquid chromatography (FPLC) systems for higher throughput and convenience. and expertise in protein biochemistry and purification make it possible to tailor the purification protocol to the characteristics and needs specific to a particular rational target. Upon determination of a rational antibacterial target and its subsequent procurement, it should be immediately and fully engaged. While multiple steps exist during such engagement, it is somewhat difficult to list a definitive order for proper execution of such and, therefore, a certain degree of adaptability is required. Nonetheless, the techniques essential for further pursuit follow, but are not in a definitive order and, instead, all have the potential to contribute a portion of all knowledge required for successful rational antibacterial drug discovery and development.

\section{Preliminary target characterization}

While every protein is different, some targets may be inherently stable and immediately easy to work with, while others prove recalcitrant to many conditions and procedures from the moment they are purified. Such intractability may prove not only inconvenient, but altogether discordant with the timeline a research team is under. Therefore, it is of the utmost benefit to characterize the target protein, and any constructs created, as soon as possible in order to move forward as efficiently as possible. As such, a number of techniques exist to aid in such hasty pursuits.

General techniques. Several miscellaneous techniques exist for the preliminary characterization of a purified target protein that may be of some service to a research team. While much information can be determined about the protein of interest from the aforementioned application of bioinformatics and literature searches, techniques exist to corroborate calculated protein parameters or, often times, determine a characteristic more precisely. Examples include, but are not limited to, isoelectric focusing to determine a precise isoelectric point that could prove highly useful during stability assessments; denatured or native polyacrylamide gel electrophoresis (PAGE) for determining sample 
purity, analyzing degradation, or evaluating dimerization; analytical gel filtration chromatography, or analytical SEC, for analyzing purity, size, dimerization, and other global characteristics of a target protein, such as whether or not it is retaining a bound cofactor or not; and mass spectrometry (MS), which offers an enormous amount of knowledge about the target, from identification via peptide fingerprinting, to de novo sequencing of an unknown target, to general structural inferences, to protein-protein interaction analyses via cross-linking MS. ${ }^{40,41}$ While only a few examples of general preliminary techniques, these highlight the importance of possessing a thorough working knowledge of protein biochemistry and the tools available for one's use in thoroughly analyzing and characterizing a target protein in early-stage antibacterial drug discovery.

Thermal shift assay. Another popular and very useful early-stage target characterization technique resides in the thermal shift assay, or differential scanning fluorimetry (DSF). This technique should be employed as early as possible as it may allow for subsequent analyses to occur that, without stable target protein, may not be feasible. Mastery of this technique is actually dually beneficial, as it can be used to identify ligands that bind to the purified target enzyme, thereby stabilizing the protein and increasing its melting temperature-which is determined by a hydrophobic fluorescent dye binding to the similarly hydrophobic innards of the target protein that are exposed upon melting and resulting in a measurable increase in fluorescence-or it can simply be used to identify salt, polyol, buffer, $\mathrm{pH}$, or additive conditions that stabilize the protein, making further work notably easier. ${ }^{42}$

\section{Compound screening and hit selection techniques}

After purified stable, homogenous target protein is acquired, one of the next rational drug discovery steps is compound screening and selection of hit compounds for the sake of assessing how "druggable" the target is - meaning how likely is it that the target is accessible to a small molecule that can elicit a measurable biological response of biological and, potentially, clinical import. Once again, an immediately initial technique worthy of noting with respect to compound screening and hit selection is simply a thorough literature review.

Active compound literature review. During early screening, it may be possible to find previous hits or compounds discovered or developed previously against related or otherwise similar targets by other research groups that may also be active against a novel rational target of interest. Even if such a compound does not show similar activity against the new target, it may prove useful as a chemical probe with utility later on in target and hit validation and characterization, or as a scaffold for iterative lead development and structure-activity relationship (SAR) analyses. Whether or not such an asset may be exhumed from the literature for any given rational drug discovery campaign, a battery of other useful drug discovery techniques prove to be absolute necessities.

Biophysical techniques. For truly novel antibacterial targets, previous inhibitors or chemical probes simply may not be known, or may not even exist. What is more, highthroughput screening (HTS) assays may not be developed, either. Also, knowledge of 
conditions necessary for the stabilization of the targeted protein may not be immediately available. Nonetheless, a reasonable starting point in the search for useful chemical probes may be found in a more medium throughput method, or even a low throughput method under the most dire of circumstances.

As mentioned above, the thermal shift assay technique is of significant utility during early stages of antibacterial drug discovery for the sake of preliminary protein characterization. While extremely useful for identifying stabilizing conditions for the novel target, the utility of the thermal shift assay in finding ligands that bind the enzyme is also substantial, but must be pursued cautiously. The reason for this is that just because a compound is discovered that binds the enzyme as a ligand, and therefore shifts the melting temperature higher, does not guarantee it has any activity, such as agonism or antagonism. As such, the discovery of such a compound would unquestionably call for further analysis and highlights the need for the use of a known functional assay or, if the existence for such does not exist, the requisite development of such.

Lower throughput techniques used for the study of ligand-protein binding interactions include surface plasmon resonance (SPR; low to medium throughput) and isothermal titration calorimetry (ITC; lowest throughput of methods discussed). SPR requires the first protein binding component be immobilized on a sensor chip as the other test-compound binding component is free in solution and passed over the first component while subtle differences in refractive index are detected at the surface of the sensor. ${ }^{43}$ ITC, on the other hand, simply uses heat as a signal to quantitatively measure binding affinity $\left(\mathrm{K}_{\mathrm{a}}\right)$, stoichiometry $(n)$, and enthalpy change $(\Delta \mathrm{H})$, after which free energy change $(\Delta \mathrm{G})$ and entropy change $(\Delta \mathrm{S})$ can be calculated. ${ }^{44}$ While lower throughput, both of these techniques benefit from the fact that neither requires labeling of any sort.

Biochemical techniques. HTS methods are desirable in order to increase efficiency in the search for hits against a rational target. Biochemical assays are naturally more amenable to supporting higher throughput screening than their biophysical counterparts. In contrast to biophysical assays, biochemical assays allow not only for the measurement of the affinity of test compounds for purified target protein, but also for the measurement of some biologically meaningful effect related to the overall function of the target enzyme. ${ }^{45}$ Moreover, biochemical assays can often be read in small volumes compliant high throughput plates and plate readers that make large numbers of repetitive assays possible, thereby introducing the possibility of screening entire libraries in very little amounts of time and covering as much chemical space as possible in the search for active compounds. Often, assays exist or can be developed that rely upon the measurement of some easily recorded change in absorbance, fluorescence, or luminescence via the exploitation of reactions involving components such as metabolic cofactors, fluorophores, and quenchers. Such assays not only allow for high throughput chemical library screening, but also allow for efficient target and hit validation. 


\section{Target and hit validation and characterization techniques}

Once hit compounds are determined, a more thorough analysis of the target enzyme and the hit compound, as well as the interactive relationship between the two, can commence. A number of techniques exist to this end, and while it may be nearly impossible for a single lab or researcher to have a mastery of all of the following techniques, as well as access to the necessary respective instrumentations, the more techniques that can be employed, the more thorough understanding and knowledge pertaining to the target will be afforded. At minimum, however, a well-balanced and complementary combination of the following techniques are necessary.

Structural biology techniques. Various structural biology techniques exist that may be used for further biophysical characterization of a target protein and any significant interactions the target protein may make with various ligands. As would be expected, each technique carries with it its own benefits and liabilities, including administrative attributes like ease of use, access, and cost, as well as actual benefits and liabilities associated with the actual nature of the techniques and the application and results that can be expected from each.

X-ray crystallography. Considered the most powerful structure-guided drug discovery tool, X-ray crystallography offers a multitude of knowledge about the target protein down to the atomic level, with some crystal structures even determined and deposited in the protein data bank (PDB) with resolution below $1 \AA{ }^{46}$ While structures at this high of a resolution are not overwhelmingly common, numerous structures exist in the PDB near the $2 \AA$ resolution level, which allows not only overall structural features to be observed, but also finer features like peptide side chains and atomic interactions critical for structure-guided drug discovery. Cons to X-ray crystallography include the need to crystallize the target protein which, depending on the protein, may take substantial amounts of time (or simply never occur), may not yield high-quality diffracting crystals, and may not necessarily represent a biologically relevant conformation of the target protein; the need for access to a high-power X-ray source; as well as the substantial learning curve involved in procuring pure, homogenous protein, attaining high resolution diffracting crystals, data collection, data processing, and refinement. Nonetheless, the benefits of X-ray crystallography continue to outweigh the costs as it remains the indisputable golden standard for protein structure analysis.

Cryo-electron microscopy. Quickly becoming one of the most popular and powerful structural biology techniques available, cryo-electron microscopy (cryo-EM) has recently undergone substantial hardware, software, and methodology improvements that allow for near-atomic resolution biomacromolecular structure determination. Benefits of cryo-EM include the fact that the target protein does not need to be crystalized, which saves a substantial amount of time for the researcher and also allows structural analysis of the protein in an arguably more "biologically relevant," or "near native" state. ${ }^{47}$ Limitations nonetheless include the requirement for pure, stable, and homogeneous protein; access to expensive equipment; substantial expertise; lower resolution structure determination relative to X-ray crystallography; and a high target 
protein molecular weight cutoff limit relative to other major structural biology techniques. ${ }^{48}$ Though these last two issues represent major historical barriers to cryo-EM, which have traditionally included difficulty crossing the $2 \AA$ resolution barrier and difficulty determining the structure of target proteins smaller than $\sim 200$ kilodaltons $(\mathrm{kDa})$, recent reports exist of instances where the technique indeed produced results that broke each of these proposed barriers. ${ }^{49}$ As such, this technique is understandably gaining momentum, offering some utility in rational drug discovery efforts, with the potential for even greater utility to come.

Nuclear magnetic resonance spectrometry. Perhaps the final main structural biology technique worth discussing, nuclear magnetic resonance (NMR) spectrometry adds a significant amount of knowledge that complements the aforementioned techniques very well. While limitations indeed exist, such as a requirement for soluble protein, access to equipment and expertise, and a less practical general maximum target protein size cutoff limit of roughly $25 \mathrm{kDa}, \mathrm{NMR}$ is an extremely powerful modern tool used in drug discovery efforts. Unlike the other techniques mentioned, NMR offers the ability to characterize highly dynamic and disordered target proteins, the ability to analyze target enzyme activity in real-time, and the ability to effectively map specific binding site characteristics, among others. ${ }^{50-52}$

Enzymology techniques. Thorough target protein characterization and validation requires a variety of different enzymology techniques. Functional biochemical assays are an absolute requirement for proper analyses of compound potency and the evaluation of mode of inhibition. A return to biophysical assays like thermal shift, ITC, and SPR is necessary for a proper assessment of binding kinetics.

\section{Iterative lead development techniques}

As the realistic terminal goal of any rational drug discovery campaign is the eventual development of lead compounds that can be carried on to clinical development, a fair amount of iterative rational compound optimization must occur. As a culmination of all pre-clinical development up to this point, it is understandable that SAR-guided chemical optimization should include all techniques heretofore discussed, as well as a few additional techniques.

SAR-guided chemical optimization. Thorough analysis of structural data, ideally with a bound ligand, affords the researcher the opportunity to visualize the target enzyme and its corresponding active or allosteric site along with a previously discovered or developed ligand. This will allow for rational modification to the compound that allows the researcher to manipulate the compound to maximize characteristics like potency via improved or added non-covalent molecular interactions, solubility via rational addition of solubilizing groups at key positions, and so on. This allows for medicinal chemistry trials to commence, where newly synthesized rationally modified compounds can be tested via in vitro or in vivo functional assays. 
Virtual screening. Additional methods can also take place once structural data is obtained, including virtual screening, where significantly large computational libraries can be prepared and screened against the target protein via techniques like docking, pharmacophore matching, and shape matching. Any possibly improved and optimized compounds that result can then be synthesized or purchased and, again, tested against the target protein for potency assessment.

Any gains or losses that result after classic "manual" SAR-guided optimization or virtual screening regarding potency and physicochemical attributes can be rationally weighed and evaluated. Then, the products can be rationally analyzed and further modified in an iterative fashion until a satisfactory product results that has, ideally, balanced an increased potency with satisfactory physicochemical properties that will not hamper the compound with respect to toxicology, metabolism, pharmacokinetics, or pharmacodynamics during future clinical trials and development.

While it is unlikely that a researcher or research team would have access to expertise and equipment needed for every technique discussed above, a working combination is required and must be tailored to the specific rational target. Pros and cons of each technique must be considered, and a respectively appropriate research plan should commence based around which techniques are needed for project success and which techniques are available. While some techniques are absolute necessities, such as bioinformatics and protein production and purification, some but not all representatives from the other overarching technique categories can be mixed and matched. For example, depending on the specific research needs, while access to SPR may not exist, ITC may take its place; while cryo-EM may not be suited for a smaller protein target, X-ray crystallography may work well; and while biochemical assays utilizing absorbance may not be feasible, development of chemical probes or substrates that utilize specific fluorophores may be a possibility. As with most endeavors, the more knowledge and expertise one possesses, the more adaptability is afforded, and the more anticipated success exists. Consequently, it is arguable that access to enough knowledge, skills, and instrumentation would allow for any novel target to be engaged.

\section{Novel Narrow Spectrum Antibacterial Targets}

Whether the novel antibacterial target of interest is intended to be a broad spectrum target, a narrow spectrum target, or simply "anything we can get," knowledge about the target itself is of paramount importance. In fact, before the actual pursuit begins, it is understood that a substantial amount of resources - time, energy, money-is going to be invested into the target. That is why, as mentioned above, a certain degree of anticipated success must accompany any real antibacterial drug discovery and development campaign. But, how can a researcher anticipate success when so little is known about a previously un-elucidated target? The only way, in fact, is to harvest as much knowledge about the target of interest in advance. This is, of course, important during rational drug discovery and development, but absolutely crucial when pursuing narrow spectrum antibacterial targets. 
Pertinent questions include, but are not limited to, is the antibacterial target essential, or does its function at least dictate a significant degree of fitness to its host organism that could be exploited in some clinically significant manner? Perhaps it is an explicitly essential enzyme target. Or, perhaps it is instead a more cryptic target that confers some degree of fitness that can be taken advantage of and leveraged in high-stress biological situations, such as during immune response, concomitant antibacterial agent administration, or human microbiome supplementation and overcrowding. With respect to potential narrow spectrum targets, another pertinent question involves the existenceor rather the absence - of the target in other bacteria that represent the beneficial members of the human microbiome. For the sake of selectivity, multiple beneficial scenarios could exist. First, it would be ideal if the target existed only in the genomes of only a few pathogens, thereby sparing beneficial bacteria from collateral damage and eradication. Alternatively, the gene responsible for the target enzyme could exist in bacteria other than the target pathogen, but perhaps the non-targeted bacteria possess bypass mechanisms that render that particular target non-essential in the respective bacteria under physiological conditions.

Other pertinent preliminary questions of course involve the overall nature of the pathogen being targeted, such as site of infection, gram-stain classification, absence or presence of efflux pumps, and extent of pathogenicity. These types of questions may help guide anticipated physicochemical characteristics necessary for any future chemical probes or inhibitors, amongst others. Altogether, it is evident that the more preliminary knowledge a researcher has before commencing a rational antibacterial drug discovery campaign, the better. Once a requisite amount of preliminary knowledge is gained, a number of actual laboratory-based techniques are required for the successful discovery and development of rational antibacterial agents.

Again, as our understanding of the human microbiome evolves, the deficiencies in traditional, broad-spectrum antibacterial therapy are becoming increasingly apparent. Both the advent of multi-drug resistant bacterial infections and the devastating disturbance of our normal bacterial ecology testify of the alarming need for novel antibacterial agents capable of selectively targeting pathogens, drug-resistant or otherwise, while mitigating the impact on beneficial and commensal organisms. As such, the benefit of narrow spectrum targets, which may afford opportunities for the killing or inhibiting specific pathogens without significantly inhibiting the growth of collateral or beneficial bacteria, is substantial. Engaging enzyme targets that meet these requirements, however, hinges upon exploiting intrinsic differences between the enzymatic proteomes of pathogenic bacteria and commensal bacteria - differences can be notably difficult to discern, thereby making the identification of such narrow spectrum antibacterial targets appropriately difficult. ${ }^{53,54}$ Two such potential targets follow.

\section{Bacterial topoisomerase I}

Bacterial topoisomerase I represents a potentially promising narrow-spectrum target as studies have arisen demonstrating its essentiality in bacterial species lacking the 
only other type IA topoisomerase (topoisomerase III). With evidence supporting the bacterial essentiality of at least one type IA topoisomerase (topoisomerase I or topoisomerase III), an overlapping role between the two enzymes, and the fact that a select few pathogenic bacterial species solely possess topoisomerase I without topoisomerase III, the catalytic inhibition of bacterial topoisomerase I represents an attractive prospect for novel, selective antibacterial development. ${ }^{55-59}$ The streptococci are one of the few bacterial species that may possess topoisomerase I as their sole type IA topoisomerase and a representative, Streptococcus mutans, exists as a particularly promising candidate for the exploration and validation of topoisomerase I as a novel, selective antibacterial target. ${ }^{60-66}$

\section{Bacterial enoyl-acyl carrier protein (ACP) reductase II, FabK}

Bacterial enoyl-acyl carrier protein (ACP) reductase II, or FabK, is an enzyme within the bacterial fatty acid biosynthesis (FAS II) pathway that represents another highly promising narrow-spectrum target. Recent studies have demonstrated the essentiality of fabk gene that codes for enoyl-ACP reductase II (FabK) in C. difficile. ${ }^{67}$ FabK is one of several disparate enoyl-ACP reductase isozymes (FabI, FabK, FabL, and FabV) that exists across bacteria. ${ }^{68-70}$ Additionally, recent data has shown that different regulatory systems control whether or not a bacterium is capable of bypassing FAS II inhibition via the uptake of exogenous fatty acids. ${ }^{71,72}$ Because FabK is the sole, essential enoyl-ACP reductase expressed in $C$. difficile, combined with the fact that different bacteria may possess an assortment of other enoyl-ACP reductase isozymes and regulatory systems, FabK from $C$. difficile represents a potential antibacterial target with particularly significant narrow-spectrum promise.

Presented here are data investigating both the bacterial topoisomerase I and FabK enzymes as novel and potentially narrow spectrum antibacterial targets via validation and characterization studies using an array of drug discovery techniques, including structural investigations, biochemical assay development and application, and inhibitor screening and testing. 


\title{
CHAPTER 2. TARGETING BACTERIAL TOPOISOMERASE $I^{2}$
}

\author{
Introduction
}

\section{Topoisomerases Background}

\section{Biological role of topoisomerases}

Topoisomerases are ubiquitous enzymes that help manage the diverse topological complications DNA undergoes during various biological processes. In general, the overall mechanism by which all topoisomerases function is centered around the initiation of controlled breaks in the DNA via temporary phosphodiester bonds between the substrate and a conserved catalytic tyrosine residue on the enzyme. ${ }^{73-75}$ The topological complications managed include negative and positive supercoils, catenated DNA rings, and various entanglements. ${ }^{75}$ There are a number of different topoisomerases that exist across all forms of life, which are organized into particular types and subtypes and serve varying biological roles. These roles may or may not overlap to a certain degree as they are carried out to address the different aforementioned topological complications in particular ways (Table 2-1). ${ }^{55,75-78}$ Type I topoisomerases exist as monomers that cause single DNA strand breaks and elicit topological change one link at a time via either a rotational or strand passage mechanism, and type II topoisomerases are multimers that require adenosine triphosphate (ATP) hydrolysis as an energy source in order to initiate double stranded breaks and facilitate DNA strand passage; whereas subtype A topoisomerases result in a 5' phosphotyrosine DNA cleavage intermediate, and subtype B topoisomerases result in 3' phosphotyrosine intermediates. ${ }^{55,75,79}$ Accordingly, topoisomerases are indispensable in their biological duties and, therefore, represent highly attractive and efficacious chemotherapeutic targets. While many human topoisomerases exist as well-validated and exploited anti-cancer targets, only a select few bacterial topoisomerases are currently exploited as useful antibacterial targets.

\section{Different bacterial topoisomerase targets}

Up to four topoisomerases can be present in the prokaryotic genome, two from the type IIA family and two from the type IA family. Type IIA topoisomerases are heterotetramers encoded by multiple genes $(\operatorname{gyr} A / B$ and $\mathrm{par} C / E)$, and type IA topoisomerases are monomers encoded by single genes-top $A$ for topoisomerase I

\footnotetext{
${ }^{2}$ Adapted from final submission with permission from Elsevier Inc. Jones, J. A., Price, E., Miller, D. \& Hevener, K. E. A simplified protocol for high-yield expression and purification of bacterial topoisomerase I. Protein Expr Purif 124, 32-40, https://doi.org/10.1016/j.pep.2016.04.010 (2016). ${ }^{73}$

Adapted from final submission with open access permission from Elsevier Inc. Jones, J. A. \& Hevener, K. E. Crystal structure of the 65-kilodalton amino-terminal fragment of DNA topoisomerase I from the grampositive model organism Streptococcus mutans. Biochem Biophys Res Commun, https://doi.org/10.1016/j.bbrc.2019.06.034 (2019. In Press). ${ }^{74}$
} 
Table 2-1. Overview of types and subtypes of select topoisomerases.

\begin{tabular}{|c|c|c|c|c|c|c|c|}
\hline Type & Subtype & Enzyme & Source & Function & Link & Energy Source & Subunits \\
\hline \multirow{7}{*}{$\begin{array}{c}\text { I, SS } \\
\text { Break }\end{array}$} & \multirow{5}{*}{ A } & Topo I & Bacteria & Relax - SCs & \multirow{5}{*}{5 , } & Torsional strain & Monomer \\
\hline & & Topo III & Bacteria & Decatenation, relax - SCs & & Torsional strain & Monomer \\
\hline & & Topoisomerase III $\alpha$ & Mammalian & Relax - SCs & & Torsional strain & Monomer \\
\hline & & Topoisomerase IIIß & Mammalian & Uncertain & & Torsional strain & Monomer \\
\hline & & Reverse gyrase & Archaea, other & Cause + SCs & & ATP & Heterodimer \\
\hline & B & Topoisomerase I & Mammalian & Various, relax $+/-\mathrm{SCs}$ & \multirow{2}{*}{$3^{\prime}$} & Torsional strain & Monomer \\
\hline & $\mathrm{C}$ & Topoisomerase $\mathrm{V}$ & Archaea & Relax $+/-$ SCs & & Torsional strain & Monomer \\
\hline \multirow{5}{*}{$\begin{array}{l}\text { II, DS } \\
\text { Break }\end{array}$} & \multirow{4}{*}{ A } & Topo II/Gyrase & Bacteria & Introduce - SCs & \multirow{5}{*}{5 , } & \multirow{5}{*}{ ATP } & Heterotetramer \\
\hline & & Topoisomerase IV & Bacteria & Relax - SCs, decatenation & & & Heterotetramer \\
\hline & & Topoisomerase II $\alpha$ & Mammalian & Decatenation & & & Homodimer \\
\hline & & Topoisomerase IIß & Mammalian & Various & & & Homodimer \\
\hline & B & Topoisomerase VI & Archaea & Relax $+/-$ SCs, decatenation & & & Heterotetramer \\
\hline
\end{tabular}

DS, double stranded; SCs, supercoils; SS, single stranded

Data sources: Champoux, J. J. DNA topoisomerases: structure, function, and mechanism. Annu Rev Biochem 70, 369-413, https://doi.org/10.1146/annurev.biochem.70.1.369 (2001). ${ }^{75}$

Pommier, Y. Drugging topoisomerases: lessons and challenges. ACS Chem Biol 8, 82-95, https://doi.org/10.1021/cb300648v (2013). Slesarev, A. I. et al. DNA topoisomerase V is a relative of eukaryotic topoisomerase I from a hyperthermophilic prokaryote. Nature 364, 735-737, https://doi.org/10.1038/364735a0 (1993). ${ }^{55}$

Wendorff, T. J. \& Berger, J. M. Topoisomerase VI senses and exploits both DNA crossings and bends to facilitate strand passage. Elife 7, https://doi.org/10.7554/eLife.31724 (2018). ${ }^{77}$ 
topB for topoisomerase III. ${ }^{55,80}$ Only two bacterial topoisomerases- the type IIA topoisomerases known as gyrase and topo IV - have inhibitors that have been developed and marketed as antibacterial drugs. ${ }^{55}$ These type IIA topoisomerases stand as very wellestablished and well-validated antibacterial targets via the quinolone class of topoisomerase poisons, which form bactericidal topoisomerase-DNA-quinolone ternary complexes and result in broad-spectrum antibacterial activity. In contrast, the type IA topoisomerases - topoisomerase I and topoisomerase III-remain relatively unexplored from a drug discovery and development standpoint and are consequently without respectively approved inhibitors. ${ }^{55}$ Current efforts are being undertaken, however, to discover and develop novel bacterial topoisomerase IA inhibitors. ${ }^{81}$

As evolutionary paralogues, topoisomerase I and topoisomerase III are believed to have arisen via gene duplication and, therefore, reasonably exemplify a certain degree of functional overlap. ${ }^{82}$ Topoisomerase I exists in all bacteria to relax negative DNA supercoils, while topoisomerase III is only found in some bacteria and is primarily geared toward decatenation (Table 2-2) ${ }^{78}$ Nevertheless, it has been shown that $E$. coli strains possessing the genes for both topoisomerase I and topoisomerase III (top $A$ and top $B$, respectively), while comparatively less fit, are able to survive top $A$ gene deletion mutations, but strains lacking the top $B$ gene showed no such viability ${ }^{83}$ The scientific deduction made herein is that when topoisomerase I activity is negated in some way, the residual topoisomerase III enzyme, if present, is capable of relieving the resultant insult, thereby maintaining viability and fitness to some extent and acting as a sort of enzymatic levee. This concept introduces the hypothesis that, on top of the potential broad-spectrum fitness cost of topoisomerase I inhibition across all species of bacteria, specific topoisomerase I inhibitors could have the added effect of showing selectivity against organisms that possess the top $A$ gene in absence of the top $B$ gene (Figure 2-1).

\section{Type IA Topoisomerases in Streptococci}

Several clinically relevant bacteria exist that possess top $A$ without top $B$ and, therefore, express topoisomerase I as their sole type IA topoisomerase. Examples include Acinetobacter baumanii, Francisella tularensis, Helicobacter pylori, Legionella pneumophila, Mycoplasma pneumoniae, and Mycobacterium tuberculosis. ${ }^{78}$ The clinically relevant streptococci were also long thought to possess top $A$ without top $B$. However, recent provisional streptococcal topoisomerase III protein sequence depositions in the National Center for Biotechnology Information (NCBI) database have surfaced that now suggest otherwise. Further bioinformatic analysis, though, suggests at least the possibility that the streptococcal topo III protein sequences found in NCBI may be misannotated as there is no NCBI-annotated streoptococcal topB gene. ${ }^{84}$ Furthermore, a Basic Local Alignment Search Tool (BLAST) nucleotide-to-nucleotide "blastn" analysis of the E. coli topB gene (accession no. CP009685.1) against the genome of the Grampositive model organism Streptococcus mutans (taxid: 1309), returned no significant similarities. ${ }^{84,85}$ Similarly, a BLAST protein-to-nucleotide "tblastn" analysis of the provisionally annotated $S$. mutans topo III protein (accession no. SUN72613.1) from 
Table 2-2. Topoisomerases present in clinically relevant bacteria.

\begin{tabular}{|c|c|c|c|c|}
\hline Species & $\begin{array}{c}\text { Topoisomerase } \\
\text { I }\end{array}$ & $\begin{array}{c}\text { Topoisomerase } \\
\text { III }\end{array}$ & Gyrase & $\begin{array}{c}\text { Topoisomerase } \\
\text { IV }\end{array}$ \\
\hline S. mutans & $\mathrm{X}$ & $?$ & $\mathrm{X}$ & $\mathrm{X}$ \\
\hline A. baumannii & $X$ & - & $\mathrm{X}$ & $X$ \\
\hline F. tularensis & $\mathrm{X}$ & - & $\mathrm{X}$ & $X$ \\
\hline H. pylori & $\mathrm{X}$ & - & $\mathrm{X}$ & - \\
\hline L. pneumophila & $\mathrm{X}$ & - & $\mathrm{X}$ & $\mathrm{X}$ \\
\hline M. pneumoniae & $\mathrm{X}$ & - & $\mathrm{X}$ & $\mathrm{X}$ \\
\hline M. tuberculosis & $X$ & - & $X$ & - \\
\hline B. anthracis & $X$ & $X$ & $X$ & $\mathrm{X}$ \\
\hline C. difficile & $\mathrm{X}$ & $\mathrm{X}$ & $X$ & - \\
\hline E. coli & $\mathrm{X}$ & $X$ & $X$ & $X$ \\
\hline E. faecalis & $X$ & $\mathrm{X}$ & $X$ & $X$ \\
\hline H. influenzae & $X$ & $X$ & $X$ & $X$ \\
\hline K. pneumoniae & $\mathrm{X}$ & $X$ & $\mathrm{X}$ & $\mathrm{X}$ \\
\hline N. gonorrhoeae & $\mathrm{X}$ & $\mathrm{X}$ & $\mathrm{X}$ & $\mathrm{X}$ \\
\hline P. aeruginosa & $\mathrm{X}$ & $X$ & $X$ & $X$ \\
\hline S. aureus & $\mathrm{X}$ & $\mathrm{X}$ & $X$ & $X$ \\
\hline$Y \cdot$ pestis & $\mathrm{X}$ & $X$ & $X$ & $X$ \\
\hline
\end{tabular}

Data source: Forterre, P., Gribaldo, S., Gadelle, D. \& Serre, M. C. Origin and evolution of DNA topoisomerases. Biochimie 89, 427-446,

https://doi.org/10.1016/j.biochi.2006.12.009 (2007). ${ }^{78}$ 


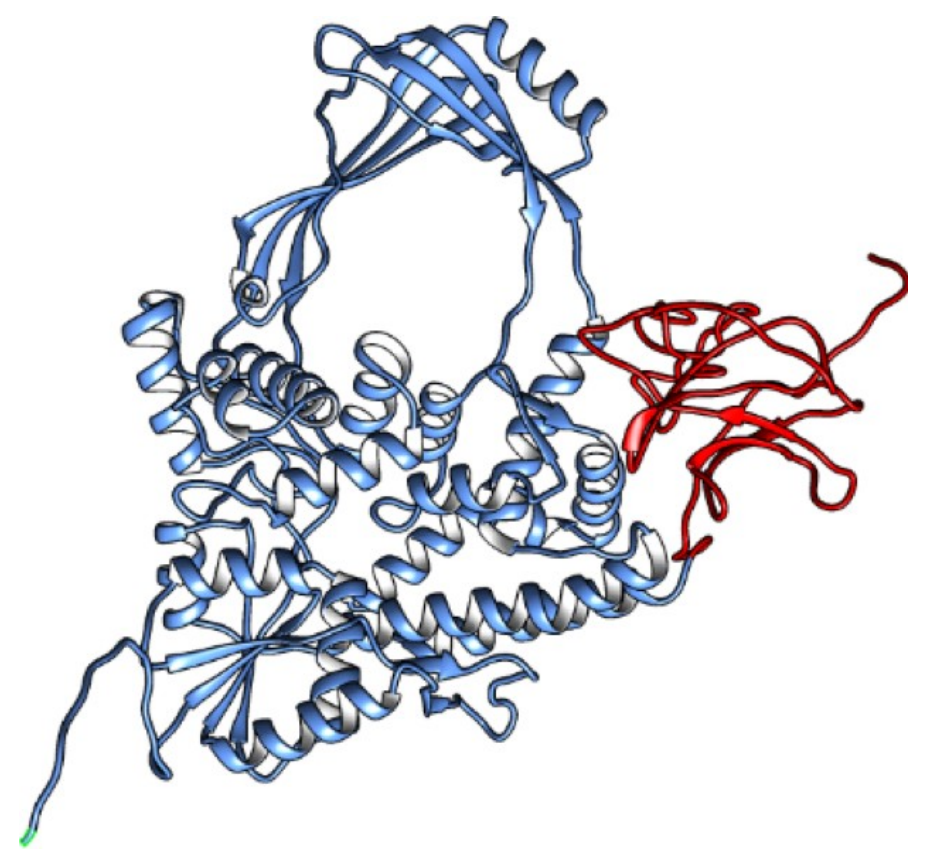

Figure 2-1. S. mutans topoisomerase I structural homology model.

Full structure of the SmTopoI enzyme (blue ribbons) with the N-term (green) and flexible C-term domain highlighted (red). 
NCBI against the $S$. mutans UA159 genome (taxid: 210007 ) only showed a $27.6 \%$ identity match with the $S$. mutans complete genome (accession no. AE014133.2), which may possibly represent a low-grade match to the nucleotide sequence for another DNAbinding enzyme from $S$. mutans such as, but not necessarily, top $A{ }^{84,85}$ An open proteinto-protein "blastp" search of the NCBI annotated $S$. mutans topo III protein (accession no. SUN72613.1), however, returned a substantial number of topo III protein sequence matches from other streptococci that range from 65.46 to $98.45 \% .{ }^{85}$ While an uncertainty indeed lingers around the presence or absence of topB in $S$. mutans, substantial utility still remains in studying the topo I enzyme from this useful organism.

If $S$. mutans does in fact possess topo I as its sole type IA topoisomerase, then it would serve as a particularly promising subject for the validation of topo I as an effective narrow-spectrum antibacterial drug target. In contrast, if it turns out $S$. mutans actually expresses topo III as well, topo I from S. mutans (SmTopoI) may, for the time being, still act as a useful surrogate antibacterial target for amassing information regarding the druggability of topo I that may later be translated to other bacteria that are confirmed to express topo I as their sole type IA topoisomerase. Therefore, either way, the investigation of SmTopoI serves as a promising head start for the characterization and validation of topoisomerase I as a selective antibacterial target. This research thereby endeavors to use $S$. mutans to address remaining gaps in knowledge relating to key structural evidence substantiating the druggability of topoisomerase I in general.

As a representative of the viridans group streptococci (VGS) and a Gram-positive model organism, $S$. mutans exists as an excellent candidate for the initial exploration and validation of topo I as a novel antibacterial target. ${ }^{60-66}$ VGS are represented by a plurality of heterogeneous streptococcal organisms that constitute a common component of the human microbiome found primarily within the oral cavity and upper respiratory tract, as well as in the gastrointestinal tract and female genitourinary tract. ${ }^{86,87}$ With historically low consideration for pathogenicity, VGS have been increasingly implicated in a wide array of problematic diseases such as endocarditis, pneumonia, cellulitis, bacteremia, and viridans streptococcal shock syndrome, among others. ${ }^{86,88}$ Moreover, VGS stand as the predominant group of microorganisms within the oropharynx, with a member of the group, $S$. mutans, representing the main culprit in dental cariogenesis. ${ }^{89}$ Considering the vast clinical and economic burdens now known to be caused by VGS, the necessity for effective antibacterials targeting these pathogens has escalated. While the majority of VGS infections are still susceptible to penicillin, the proliferation of drug-resistant VGS organisms, including those resistant to penicillins, macrolides, and lincosamide antibiotics, is becoming more of a concern. ${ }^{86,87}$ Additionally, as the beneficial bacteria that cohabitate the usual sites of VGS residence are concomitantly eradicated by the traditional use of these broad-spectrum antibacterials, significant collateral consequences such as candidiasis and Clostridioides difficile infections have increased over time. ${ }^{90-92}$ Therefore, novel targets for new VGS-focused antibacterials are in dire need. 


\section{Targeting SmTopoI}

As most bacterial topo I inhibitors published to date are general DNA-binding agents, they would be expected to show poor selectivity for individual type IA topoisomerases. ${ }^{56}$ With that in mind, there are two known direct mechanisms for topoisomerase inhibition. First is the formation of aforementioned cytotoxic "topoisomerase poisons" that form ternary enzyme-DNA-inhibitor complexes and prevent the rejoining of transiently cleaved DNA strands. The second mechanism is catalytic inhibition via competition at the allosteric ATP site, or direct competition at the active DNA site. As type IA topoisomerases act independently of ATP, any catalytic inhibition would likely need to be accomplished by specifically targeting the DNA active site. While the majority of published direct bacterial topoisomerase I inhibitors have been shown to be "topoisomerase poisons," scientific precedent does exist for catalytic bacterial topoisomerase I inhibition via direct DNA competition. ${ }^{93,94}$ As such, this research focuses on characterizing the DNA active site of SmTopoI as the first step in rationally providing evidence of the possibility of selective topoisomerase I druggability. Accordingly, a thorough structural understanding of the target enzyme is required to identify key determinants of substrate and inhibitor binding along the DNA active site. Such knowledge would support future efforts for the identification of novel chemical probes and support the druggability of bacterial topoisomerase I. To gain such an understanding, the structural determination of the enzyme at atomic resolution is necessary. Substantial initial hurdles exist, however, as topo I is a DNA binding enzyme and, as such, has been proven throughout the literature to be a highly difficult target enzyme to purify and crystallize. ${ }^{95-97}$

Unfortunately, additional difficulties also exist that are unique to targeting topo I. For example, unlike the ATP-dependent type IIA topoisomerases, the ATP-independent type IA topoisomerases catalyze an energetically favorable event and rely solely on the torsional strain of their DNA substrate to generate the energy required to carry out their biological responsibilities relaxing hyper-negative supercoils or decatenating interlinked DNA. ${ }^{55}$ This fact carries with it multiple liabilities. First, unlike the type IIA topoisomerases, there is no opportunity to measure the expenditure of an energy source when targeting type IA topoisomerases. As such, the only way to measure the activity of the topo I enzyme, and in turn measure the inhibition of its activity, is by measuring the degree of substrate DNA supercoiling that remains after incubation.

\section{Aims}

\section{Protein Expression and Purification of SmTopoI}

As mentioned above, inherent difficulties are involved with the purification of DNA-binding proteins such as bacterial topoisomerase I. The initial need to separate cellular DNA from the target enzyme introduces an early obstacle in the protocol. To overcome such an obstacle, previous methods including treating extracts to batch anion 
exchange, DNase treatments, and DNA precipitations using different compounds like streptomycin sulfate and polyethyleneimine have been employed. ${ }^{98}$ While each DNAseparation method has its own advantages and disadvantages, they all share a certain degree of costliness with respect to time and resources. Previous studies regarding the expression and purification of bacterial topoisomerase I have yielded varied results. While truncated N-terminal fragments of $E$. coli topoisomerase I have been well studied and characterized, the full-length enzyme is desirable for thorough characterization and inhibitor development. ${ }^{99,100}$ In one study, the purification and analysis of full-length topoisomerase I from the extremophile Thermotoga maritima was carried out (expressed in E. coli with IPTG induction; heated to precipitate non-thermophile proteins and DNA; purified using a heparin column followed by size exclusion chromatography) and yielded 2-3 mg/Liter. ${ }^{101,102}$ However, information gleaned from an extremophile would not necessarily be expected to provide data that could be reliably applied to clinically relevant bacteria. Another study detailed the purification of full-length topoisomerase I from Streptococcus pneumonia (expressed in E. coli with IPTG induction followed by dialysis, IMAC purification, and a second dialysis step), but did not report yield. ${ }^{93}$ Despite their noteworthy contributions to our understanding of bacterial topoisomerase I, these studies either failed to report the amount of protein yielded from their respective protocols, or the yield was relatively low, making biophysical and biochemical assay studies logistically difficult. A study developing the purification of topoisomerase I from $E$. coli yielded a significant amount of the enzyme, but would not be appropriate for expressing and purifying topoisomerase I from non-E.coli bacteria as the protocol lacked a method for separating non-native enzyme from native. ${ }^{103}$ More recent studies discussed the purification of full-length E. coli and Mycobacterium tuberculosis topoisomerase I used for the subsequent crystallization and characterization of the respective enzymes, but did not report the yields. ${ }^{95,96}$

Herein, the expression, rapid two-step purification, and preliminary characterization of full-length topo I from $S$. mutans with yields significantly greater than previously reported purifications of bacterial topoisomerase I are reported. Notably, the expression protocol requires significantly less effort than classical induction methods and the purification protocol bypasses traditionally cumbersome DNA-removal methods and can be carried out in a single day with an estimated purity of $95 \%$ or higher.

\section{Structural Determination of SmTopoI_N65}

As overall interest in type IA topoisomerases continues to increase, especially with regard to efforts aimed at discovering and developing novel antibacterial agentsparticularly topo I inhibitors - the need for a greater amount of structural topo I data becomes more apparent. ${ }^{56,57,93,97,104}$ Aiding in these efforts, several three-dimensional apo- and co-crystal structures of topo I from various bacterial species have been determined, including those from the gram-negative model organism E. coli (EcTopoI) ${ }^{96}$ the gram-negative extremophile Thermotoga maritima (TmTopoI), ${ }^{101}$ and the uniquely non-gram-classified Mycobacterium tuberculosis (MtTopoI). ${ }^{95}$ 
Though E. coli prevails as the predominant model organism in microbiological research, inherent differences exist between gram-negative and gram-positive bacteria. Accordingly, as Streptococcus mutans has gained utility as a gram-positive model organism, ${ }^{105}$ efforts were undertaken to obtain diffraction-quality full-length S. mutans topo I (SmTopoI) protein crystals. Though, similar to other topo I crystallization campaigns reported in the literature, such efforts were met with initial difficulty. ${ }^{95,96}$ Therefore, rational constructs of main body N-terminal fragment of SmTopoI were cloned and expressed to remove the flexible $\mathrm{C}$-terminal domain and improve chances of crystallization. This eventually led to a 65-kDa fragment (SmTopoI_N65) that yielded high diffraction-quality crystals used for structural characterization of SmTopoI. Herein the three-dimensional crystal structure of SmTopoI_N65, the first topo I structure determined from a gram-positive bacterium and its corresponding structural characterization are reported.

\section{Materials and Methods}

\section{Reagents, Chemicals, Biologicals, and Equipment}

Biological materials, equipment, and reagent-grade chemicals used were from the following sources: Streptococcus mutans genomic DNA, ATCC® 700610D-5 ${ }^{\mathrm{TM}}$ from ATCC ${ }^{\circledR}$ (Manassas, VA); ArcticExpress(DE3) Competent Cells, pUC18 plasmid, XL1Blue Supercompetent cells, and BL21(DE3)-Gold Competent cells from Agilent Technologies (Santa Clara, CA); Champion ${ }^{\mathrm{TM}}$ pET SUMO Protein Expression System, including One Shot ${ }^{\circledR}$ Mach $1^{\mathrm{TM}}-\mathrm{T} 1^{\mathrm{R}}$ and BL21(DE3) One Shot ${ }^{\circledR}$ Chemically Competent E. coli cells from invitrogen ${ }^{\mathrm{TM}}$ by Life Technologies ${ }^{\mathrm{TM}}$ (Carlsbad, CA); HyperLadder ${ }^{\mathrm{TM}}$ $1 \mathrm{~kb}$ Plus from Bioline USA Inc (Taunton, MA); $\mathrm{pET}-15 \mathrm{~b}$ vector, $\mathrm{pET}$-16b vector, and pET-21d vector from Novagen ${ }^{\circledR}$ EMD Millipore ${ }^{\circledR}$ (Billerica, MA); Quick Ligation ${ }^{\mathrm{TM}}$ Kit, BamHI, NdeI, and NcoI restriction enzymes from New England Biolabs, Inc. (Ipswich, MA); Difco ${ }^{\mathrm{TM}}$ LB, Miller and Terrific Broth from BD ${ }^{\mathrm{TM}}$ (Franklin Lakes, NJ); Thermo Scientific ${ }^{\mathrm{TM}}$ GelCode ${ }^{\mathrm{TM}}$ Blue Safe Protein Stain and Spectra ${ }^{\mathrm{TM}}$ Multicolor High Range Protein Ladder from Thermo Fisher Scientific (Waltham, MA); HiMark ${ }^{\mathrm{TM}}$ Prestained Protein Standard, Novex ${ }^{\mathrm{TM}}$ InVision ${ }^{\mathrm{TM}}$ His-Tag In-Gel Stain, Platinum ${ }^{\circledR}$ PCR SuperMix High Fidelity, NuPAGE® Novex ${ }^{\circledR} 3-8 \%$ Tris-Acetate Protein Gels, Novex ${ }^{\mathrm{TM}}$ NuPAGE ${ }^{\mathrm{TM}}$ LDS Sample Buffer (4X), and Novex ${ }^{\mathrm{TM}}$ NuPAGETM $^{\mathrm{TM}}$ Tris-Acetate SDS Running Buffer and Molecular Probes ${ }^{\mathrm{TM}} \mathrm{SYPRO}^{\mathrm{TM}}$ Orange Protein Gel Stain from Invitrogen $^{\mathrm{TM}}$ by Life Technologies ${ }^{\mathrm{TM}}$ (Carlsbad, CA); QIAGEN Plasmid Maxi Kit, QIAquick PCR Purification Kit, and QIAprep Spin Miniprep Kit from Qiagen (Valencia, CA); DTT, glycerol, IPTG, lysozyme, $\mathrm{NaCl}, \mathrm{MgCl}_{2}$, protease inhibitor tablets (EDTAfree), Tris, Tris base, and Triton X-100 from Fisher Scientific (Pittsburgh, PA);DNase I from Worthington Biochemical Corporation (Lakewood, NJ); ampicillin, kanamycin, and $\mathrm{KH}_{2} \mathrm{PO}_{4}$ from Acros Organics, part of Thermo Fisher Scientific (Waltham, MA); 30,000 MWCO Amicon ${ }^{\mathrm{TM}}$ Ultra-15 Centrifugal Filter Units and Imidazole from EMD Millipore ${ }^{\circledR}$ (Billerica, MA); $\mathrm{K}_{2} \mathrm{HPO}_{4}$ from Sigma Aldrich (St. Louis, MO); GelRed ${ }^{\mathrm{TM}}$ nucleic acid gel stain from Biotium, Inc. (Hayward, CA); primers synthesized by 
Integrated DNA Technologies (Coralville, IA); Hampton Solubility \& Stability Screen

$2^{\mathrm{TM}}$ from Hampton Research (Aliso Viejo, CA). ÄKTA Purifier FPLC, HisTrap-HP 5 mL column, and HiLoad 26/600 Superdex 200 PG size exclusion column from GE Healthcare Life Sciences (Pittsburgh, PA).

\section{Buffers}

The mini-expression lysis buffer is composed of $75 \mathrm{uL}$ of $1 \% \mathrm{n}$-octyl beta-Dthioglucopyranoside (OTG) in $20 \mathrm{mM}$ Tris $\mathrm{HCl}(\mathrm{pH} 7.5), 0.1 \mathrm{mg}$ Lysozyme, $0.1 \mathrm{mg}$ DNase I, and $0.5 \mathrm{uL}$ of $0.5 \mathrm{M} \mathrm{MgCl}_{2}$ for one cell pellet. The $2 \mathrm{x}$ LDS dye is composed of $5 \mathrm{~mL}$ Novex $^{\mathrm{TM}}$ NuPAGE ${ }^{\mathrm{TM}}$ LDS Sample Buffer (4X), $500 \mathrm{mM}$ DTT, and brought to 10 $\mathrm{mL}$ with $\mathrm{ddH}_{2} \mathrm{O}$. The standard lysis buffer is composed of $20 \mathrm{mM}$ sodium phosphate $(\mathrm{pH}$ 7.4), one Pierce ${ }^{\circledR}$ EDTA-free protease inhibitor tablet per $50 \mathrm{~mL}, 1 \mathrm{mM} \mathrm{DTT}, 0.5 \%$ Triton X, $0.5 \mathrm{M} \mathrm{NaCl}, 5 \%$ glycerol, $0.5 \mathrm{mg} / \mathrm{mL}$ lysozyme, $5 \mu \mathrm{g} / \mathrm{mL}$ DNase, and $5 \mathrm{mM}$ imidazole. Lysis buffer 2 is composed of standard lysis buffer without DNase I. Buffer A is composed of $20 \mathrm{mM}$ sodium phosphate ( $\mathrm{pH} 7.4), 0.5 \mathrm{M} \mathrm{NaCl}, 5 \mathrm{mM}$ imidazole, and $5 \%$ glycerol. Buffer B is composed of $20 \mathrm{mM}$ sodium phosphate $(\mathrm{pH} 7.4), 0.15 \mathrm{M} \mathrm{NaCl}$, $250 \mathrm{mM}$ imidazole, and 5\% glycerol. The size exclusion buffer is composed of $50 \mathrm{mM}$ sodium phosphate ( $\mathrm{pH} 7.4$ ) and $150 \mathrm{mM} \mathrm{NaCl}$. Buffer $\mathrm{C}$ is composed of buffer A with $1 \mathrm{M} \mathrm{NaCl}$. Buffer $\mathrm{D}$ is composed of buffer $\mathrm{B}$ with $0.5 \mathrm{M} \mathrm{NaCl}$ and $350 \mathrm{mM}$ imidazole. Size exclusion buffer 2 is composed of $20 \mathrm{mM}$ Tris ( $\mathrm{pH} 8.0$ ), $1 \mathrm{mM} \mathrm{MgCl}, 0.5 \mathrm{M} \mathrm{NaCl}$, and $1 \mathrm{mM}$ DTT. The gel assay buffer and assay stop buffer were prepared as previously reported. ${ }^{104}$ The gel assay buffer is composed of $10 \mathrm{mM}$ Tris $\mathrm{pH} 8.0,50 \mathrm{mM} \mathrm{NaCl}$, and 6 $\mathrm{mM} \mathrm{MgCl} 2$. The assay stop buffer is composed of 50mM EDTA, 50\% glycerol, and $0.5 \% \mathrm{w} / \mathrm{v}$ bromophenol blue.

\section{SmTopoI Cloning and Plasmid Construction}

Initially, the top $A$ gene was cloned from Streptococcus mutans UA159 genomic DNA for insertion into the $\mathrm{pET}-15 \mathrm{~b}$ vector based for the NdeI and BamHI restriction sites using the following primers:

TopA_Forward: 5'-TGT AGA CAT ATG ACA AGT AAA ACA ACG ACA ACA-3'

\section{TopA_Reverse: 5'-TCA TCA GGA TCC TTA TTT AAC AGC TTT TTC CTT-3'}

PCR was carried out via Platinum ${ }^{\circledR}$ PCR SuperMix High Fidelity protocol and denatured at $94{ }^{\circ} \mathrm{C}$ for 30 seconds, annealed at $58{ }^{\circ} \mathrm{C}$ for 40 seconds, and extended at $68{ }^{\circ} \mathrm{C}$ for two minutes for 30 cycles and then held at $4{ }^{\circ} \mathrm{C}$. Success was analyzed via $1 \%$ agarose gel electrophoresis based upon the confirmed presence of the $\sim 2 \mathrm{Kbp}$ SmTopA_pET PCR product. SmTopA_pET was then purified using QIAQuick ${ }^{\circledR}$ PCR Purification Kit per protocol. The $\mathrm{pET}-15 \mathrm{~b}$ vector was then digested with NdeI and BamHI restriction enzymes per New England Biolabs (NEB) protocol, purified, then mixed and ligated with the SmTopA_pET PCR product via NEB Quick Ligation ${ }^{\mathrm{TM}}$ Kit protocol. The ligation 
product (SmTopA15b) was then transformed into XL1-Blue Supercompetent cells per protocol. Upon successful transformation, the plasmid was isolated with the QIAprep Spin Miniprep Kit via protocol and the sequence was confirmed via Retrogen, Inc. (San Diego, CA). The plasmid was also transformed into BL21(DE3)-Gold Competent cells via protocol.

The top $A$ gene was sub-cloned from the $\mathrm{pET}-15 \mathrm{~b}$ vector in XL-1 Blue into the pET-16b vector using NdeI and BamHI restriction enzymes and NEB Quick Ligation ${ }^{\mathrm{TM}}$ Kit per manufacturer protocols.

For the pET 21d vector, the SmTopA gene was amplified via PCR around NcoI and BamHI restriction sites using the following primers:

\author{
NcoI_Site_For: TAT ACG ATG GGC AGC AGC CAT CAT C \\ NdeI_Site_For: GGC AGC CCC ATG GCA AGT AAA AC \\ BamHI_Site_Rev: TTC GGA TCC TTT TTA ACA GCT TTT TCC TTA TAG TCA \\ $\mathrm{CAC}$
}

PCR was carried out via Platinum ${ }^{\circledR}$ PCR SuperMix High Fidelity protocol and denatured at $94^{\circ} \mathrm{C}$ for 30 seconds, annealed at $58^{\circ} \mathrm{C}$ for 45 seconds, and extended at $68^{\circ} \mathrm{C}$ for two minutes and thirty seconds for 35 cycles and then held at $4{ }^{\circ} \mathrm{C}$.

For the pET-SUMO vector, the topA gene was cloned from PCR with "hanging $\mathrm{T}^{\text {" ends per Champion }}{ }^{\mathrm{TM}}$ pET SUMO Protein Expression System protocol using the following primers:

\title{
TopA_SUMO_For: AGC ACA AGT AAA ACA ACG ACA ACA GTA AAA AAG ACG
}

\section{TopA_SUMO_Rev: TTA TTT AAC AGC TTT TTC CTT ATA GTC ACA CTC CTT ATT GC}

PCR was carried out via Platinum ${ }^{\circledR}$ PCR SuperMix High Fidelity protocol and denatured at $94^{\circ} \mathrm{C}$ for 30 seconds, annealed at $60^{\circ} \mathrm{C}$ for 45 seconds, and extended at $68^{\circ} \mathrm{C}$ for two minutes and thirty seconds for 35 cycles and then held at $4{ }^{\circ} \mathrm{C}$.

All gene and respective plasmids were ligated and transformed per respective protocols per respective protocols into XL1-Blue Supercompetent cells and BL21(DE3)Gold Competent cells except the SmTopA_SUMO plasmid used with the Champion ${ }^{\mathrm{TM}}$ pET SUMO Protein Expression System, which utilized included One Shot ${ }^{\circledR}$ Mach1 $1^{\mathrm{TM}}$ $\mathrm{T}^{\mathrm{R}}$ and BL21(DE3) One Shot ${ }^{\circledR}$ Chemically Competent E. coli cells. Sequences were confirmed by sequencing via Retrogen, Inc. (San Diego, CA). 


\section{Mini-Expression of SmTopI15b}

A mini-expression was initially conducted in order to determine preliminary expression conditions, starting with a $5-\mathrm{mL} \mathrm{LB}$ and $100 \mathrm{ug} / \mathrm{mL}$ starter culture of SmTopI15b in BL21(DE3)-Gold grown at $32^{\circ} \mathrm{C}$ overnight. Upon the next day, the sample was diluted 100 -fold into three $5-\mathrm{mL}$ sterile $\mathrm{TB}$ and $100 \mathrm{ug} / \mathrm{mL}$ ampicillin samples for each of three different temperatures: $37^{\circ} \mathrm{C}, 25^{\circ} \mathrm{C}$, and $18^{\circ} \mathrm{C}$. Cultures were then grown for two hours, then one sample from each temperature was induced with $1 \mathrm{mM} \mathrm{IPTG}$, one with $0.5 \mathrm{mM}$ IPTG, and one remained un-induced. Samples were then grown an additional twenty-four hours, then centrifuged at 10,000 rpm for five minutes. The supernatant was then removed and each pellet was dissolved in Mini_Lysis_Buffer for five minutes. Cells were re-suspended and vortexed for 30 seconds to completely dissolve and the samples were centrifuged at 13,000 rpm for five minutes. The supernatants were then separated into clean $1.5 \mathrm{~mL}$ microcentrifuge tubes as the soluble fractions. Pellets were washed by re-suspending in $30 \mathrm{uL}$ of $1 \%$ OTG in $20 \mathrm{mM}$ Tris $(\mathrm{pH}$ 7.5) and centrifuging at 13,000 rpm for five minutes. The supernatant was then discarded. Pellets were once again re-suspended in $30 \mathrm{uL}$ of $1 \%$ OTG in $20 \mathrm{mM}$ Tris $(\mathrm{pH} \mathrm{7.5)}$ and mixed with $30 \mathrm{uL}$ of LDS_Sample_Buffer, then centrifuged again at 13,000 rpm for five minutes with the resulting supernatant containing the insoluble fraction. $10 \mathrm{uL}$ samples were then heated at $95^{\circ} \mathrm{C}$ for five minutes and loaded on SDS-PAGE gels for analysis. Mini-expression was repeated for 48-hour growths as well. (Data not shown.) Miniexpression was again repeated with $\mathrm{SmTopI} 16 \mathrm{~b}$ at $18^{\circ} \mathrm{C}$.

\section{Expression of SmTopoI constructs and cell lysis}

Expression and purification experiments were conducted in duplicate. SmTopI15b initial starter cultures were grown in BL21 cell lines at $32^{\circ} \mathrm{C}$ overnight in $5-\mathrm{mL}$ LB with $100 \mathrm{ug} / \mathrm{mL}$ ampicillin (pET constructs) or $50 \mathrm{ug} / \mathrm{mL}$ kanamycin (SUMO construct) and used to inoculate $500 \mathrm{~mL}$ TB with respective antibiotics. Cultures were grown to OD600 $\sim 0.6$, induced with $0.5 \mathrm{mM}$ IPTG, and expressed at $18^{\circ} \mathrm{C}$ for 24 hours. Auto-induction was carried out per Studier protocol with SmTopI16b and grown for 24 hours at $37^{\circ} \mathrm{C} .{ }^{106}$ All samples were then centrifuged at $15,000 \mathrm{~g}$ for 30 minutes at $4^{\circ} \mathrm{C}$ and the supernatants were discarded. Pellets were stored at $-80^{\circ} \mathrm{C}$.

Cell pellets for all constructs were thawed at room temperature and immediately lysed in the standard lysis buffer by stirring for 1 hour at $4^{\circ} \mathrm{C}$. Samples were then sonicated for 8 minutes, centrifuged at $18 \mathrm{~K} \mathrm{rpm}$ at $4^{\circ} \mathrm{C}$ for 15 minutes, filtered at 0.22 um, and collected. Auto-induction samples were lysed in the same fashion, except lysis buffer 2 was used, and then samples were sonicated and filtered as above.

\section{Purification of SmTopoI constructs}

Each initial construct sample was purified using a HisTrap-HP $5 \mathrm{~mL}$ column on an ÄKTA Purifier FPLC. Samples were loaded at $1 \mathrm{~mL} / \mathrm{min}$, washed with 20 column 
volumes (CVs) Buffer A at $2.5 \mathrm{~mL} / \mathrm{min}$, and eluted via linear gradient from $100 \%$ Buffer A to $100 \%$ Buffer B over $20 \mathrm{CVs}$ at $2.5 \mathrm{~mL} / \mathrm{min}$. Samples were then collected and concentrated using a 30,000 MWCO Amicon ${ }^{\mathrm{TM}}$ Ultra-15 Centrifugal Filter Unit. The SmTopI16b sample was collected and loaded onto a GE HiLoad 26/600 Superdex 200 PG size exclusion column and eluted with 1.5 CV of SE Buffer. The sample expressed via auto-induction (SmTopI16b_AI) was purified similarly, except it was loaded and washed with Buffer $\mathrm{C}$ for $40 \mathrm{CV}$ s and eluted with a step gradient of Buffer D using 7CV steps at $15 \%, 20 \%$, and $100 \%$ with the sample eluting in a sharp peak in the $100 \%$ step. The sample was then loaded onto a pre-equilibrated GE HiLoad 26/600 Superdex 200 PG size exclusion column and eluted with SE Buffer 2. All sample concentrations were attained via Bradford assay with BSA as the standard.

\section{Biochemical Assays}

Gel assays were used to confirm target protein activity using published methods. ${ }^{104}$ The pUC18 plasmid was isolated via QIAGEN Plasmid Maxi Kit per protocol. Enzyme assays were conducted in standard volumes of $20 \mu \mathrm{L}$ comprised of 25 , $50,75,100,150,200$, or $400 \mathrm{ng}$ SmTopI; 500 ng negatively supercoiled pUC18 plasmid; and a sufficient quantity of gel assay buffer to bring the reaction volume to $20 \mathrm{uL}$. One unit of commercially obtained E. coli topoisomerase I was substituted for SmTopI as a positive control. Assays were incubated at $37^{\circ} \mathrm{C}$ for 30 minutes and then terminated using $5 \mu \mathrm{L}$ of assay stop buffer per reaction. DNA relaxation activity was monitored on $0.7 \%$ agarose gels in Tris-Acetate-EDTA TAE buffer at $20 \mathrm{~V}$ for 10 hours and then stained with GelRed ${ }^{\mathrm{TM}}$ nucleic acid gel stain per protocol. Freeze trials were conducted similarly using $1 \mathrm{ug}$ (high concentration) or $400 \mathrm{ng}$ (low concentration) of enzyme in $50 \%$ glycerol or $0 \%$ glycerol and either snap-frozen in liquid nitrogen, frozen drop-wise in liquid nitrogen and stored at $-80^{\circ} \mathrm{C}$ overnight, or simply refrigerated. Non-specific DNAse activity assay was conducted using published methods and incubated for 6 hours. ${ }^{103}$

\section{Thermal Stability Assay}

Protein stability and solubility conditions were analyzed via the use of Hampton Research Solubility and Stability Screen $2^{\mathrm{TM}}$ per protocol to evaluate buffers and salt.

\section{Peptide fingerprinting}

Target protein identity was confirmed by standard in-gel tryptic digest and LCMS analysis. ${ }^{107-109}$ Peptide spectra matching and protein identification was achieved by database search using Sequest HT algorithm in Proteome Discoverer 1.4 (Thermo Scientific). 


\section{SmTopoI Protein Homology Modeling}

A homology model of the SmTopoI protein (Figure 2-1) was built using the $E$. coli $67-\mathrm{kDa}$ N-term fragment (1ECL) and T. maritima (2GAI) topoisomerase I crystal structures as templates, the Streptococcus mutans (UA159 strain) protein sequence, and the program MODELLER. ${ }^{110,111}$

\section{SmTopoI_N65 Cloning, Expression, Purification and Crystallization}

SmTopoI_N65 was cloned, expressed, and purified to over 95\% purity via PAGE analysis similarly to full-length SmTopoI described elsewhere. ${ }^{73,74}$ However, the target construct gene of interest, top $A_{-} N 65$, was amplified via PCR off of the top $A$-containing SmTopI_16b plasmid from above based around the NdeI and BamHI restriction sites using the following primers:

TopA_Forward: 5'-TGT AGA CAT ATG ACA AGT AAA ACA ACG ACA ACA G-3'

\section{TopA_N65_Rev: 5’-GGA TGG ATC CTT ACT GTT CCT CTG C-3’}

The gene was ligated into a pET-15b vector $\left(\right.$ Novagen ${ }^{\circledR}$ EMD Millipore ${ }^{\circledR}$, Billerica, MA) and the target plasmid was transformed into BL21(DE3)-Gold Competent cells (Agilent Technologies, Santa Clara, CA) per the manufacturer's specified protocol. The target enzyme was expressed via auto induction and purified per previous protocol, except Tris was substituted for all phosphate buffers. ${ }^{73,106}$ Briefly, cells were lysed for 1 hour at $4^{\circ} \mathrm{C}$ in Lysis Buffer composed of $20 \mathrm{mM}$ Tris-hydrochloride (HCl) $\mathrm{pH}$ 8.0, one Pierce ${ }^{\circledR}$ EDTA-free protease inhibitor tablet per $50 \mathrm{~mL}, 1 \mathrm{mM}$ DTT, $0.5 \%$ Triton X, 0.5 $\mathrm{M} \mathrm{NaCl}, 5 \%$ glycerol, $0.5 \mathrm{mg} / \mathrm{mL}$ lysozyme, and $5 \mathrm{mM}$ imidazole, then sonicated, centrifuged, and filtered at $0.22 \mu \mathrm{m}$. Hexa-his-tagged protein was purified using a HisTrap-HP $5 \mathrm{~mL}$ column on an ÄKTA Purifier FPLC (GE Healthcare Lifesciences, Pittsburgh, PA) via step-gradient nickel immobilized metal affinity chromatography (IMAC) in Buffer A, composed of $20 \mathrm{mM}$ Tris- $\mathrm{HCl} \mathrm{pH} \mathrm{8.0,1} \mathrm{M} \mathrm{NaCl}, 5 \mathrm{mM}$ imidazole, and 5\% glycerol, and Buffer B, composed of $20 \mathrm{mM}$ Tris- $\mathrm{HCl} \mathrm{pH} 8.0,0.5 \mathrm{M} \mathrm{NaCl}, 350$ $\mathrm{mM}$ imidazole, and 5\% glycerol. The sample was then purified on a GE HiLoad 26/600 Superdex 200 PG size exclusion chromatography (SEC) column in SEC Buffer, composed of $20 \mathrm{mM}$ Tris ( $\mathrm{pH} \mathrm{8.0),} 1 \mathrm{mM} \mathrm{MgCl}_{2}, 0.5 \mathrm{M} \mathrm{NaCl}$, and $1 \mathrm{mM}$ DTT. Target protein fractions were pooled, concentrated with a 10,000 MWCO Amicon ${ }^{\mathrm{TM}}$ Ultra-15 Centrifugal Filter Unit from EMD Millipore ${ }^{\circledR}$ (Billerica, MA) and used for crystallography.

SmTopoI_N65 was crystallized at $4.6 \mathrm{mg} \mathrm{mL}^{-1}$ in a $3 \mathrm{uL}: 3 \mathrm{uL} 1: 1$ ratio protein to condition using the hanging-drop vapor-diffusion method off of a coarse-matrix screen. The initial crystal growth condition was JCSG-plus (Molecular Dimensions, Maumee, $\mathrm{OH})$ condition $1-5(0.2 \mathrm{M}$ magnesium formate dihydrate, $20 \% \mathrm{w} / \mathrm{v}$ PEG 3350$)$ at $18^{\circ} \mathrm{C}$. A 1:1 mixture of Izit Crystal Dye (Hampton Research, Aliso Viejo, CA) and acid red dye was added to original crystal-containing drops and allowed to stain overnight for preliminary crystal assessment. Unstained crystals from the same well, but from a 
different drop, were collected for X-ray diffraction analysis. Initial crystals showed relatively weak diffraction (greater than $4 \AA$ ). Optimized crystals were then obtained via fine grid screen in $0.2 \mathrm{M}$ magnesium formate dihydrate, $25 \% \mathrm{w} / \mathrm{v}$ PEG 3350 at $4{ }^{\circ} \mathrm{C}$ and unstained crystals were collected for analysis. Crystals were grown in 48-well VDX plates with sealant (Hampton Research, Aliso Viejo, CA) and were visible within two weeks.

\section{SmTopoI_N65 Structure Determination and Refinement}

SmTopoI_N65 crystals were harvested, looped through 20\% w/v PEG 3350/30\% MPD in the crystallization condition for cryo-protection, and cooled to $100 \mathrm{~K}$ for data collection. A complete data set was collected at GM/CA-CAT beamline 23ID-D of sector 23 of the Advanced Photon Source (APS), Argonne National Laboratory, Lemont, Illinois, USA (Table 2-5). Data were processed and scaled, then the structure of SmTopoI_N65 was determined via molecular replacement using the previously determined 67-kDa N-terminal fragment of EcTopoI (PDB 1ECL) ${ }^{100}$ all using the $H K L$ 3000 suite. ${ }^{112}$ Initial automatic model building was performed within $H K L-3000$ using Buccaneer. ${ }^{12,113}$ Refinement was then conducted using phenix.refine and the model was further built out using AutoBuild from the PHENIX suite. ${ }^{114}$ Subsequent refinement and model building was conducted iteratively with phenix.refine and Coot. ${ }^{14,115}$ Late-stage automated re-refinement was conducted using PDB REDO. ${ }^{116}$ Validation of the structure was conducted within PHENIX using MolProbity. ${ }^{117}$ Figure 2-14 was produced with the UCSF Chimera package. ${ }^{118}$

\section{TopoI Sequence and Structure Alignments}

SmTopoI_N65, EcTopoI_N67, EcTopoIII (PDB 1D6M), and MtTopoI sequence alignment was generated with the ESPript 3 server (http://espript.ibcp.fr/) using a multiple protein sequence alignment produced with Clustal Omega, with secondary structure information extracted from the SmTopoI_N65 structure. ${ }^{119,120}$ Residues within $5 \AA$ of the active site Y316 were determined with UCSF Chimera and the structure distances measurement tool. ${ }^{118}$ Structural alignment of SmTopoI_N65 with EcTopoI_N67 (PDB 1ECL) and MtTopoI (PDB 5UJ1) conducted with UCSF Chimera MatchMaker structure comparison tool. ${ }^{100,101,118}$

\section{Generation of SmTopoI_N65 Topology Model}

The SmTopoI_N65 topology model was generated with PDBsum and SmTopoI_N65.pdb structure file. ${ }^{1 \overline{2} 1}$ 


\section{SmTopoI_N65 Multiple Sequence Alignment with Select Topo I Homologs of Undetermined Structure}

SmTopoI_N65, EcTopoI_N67, and MtTopoI sequence alignment was generated similarly as done described in the main manuscript with the ESPript 3 server (http://espript.ibcp.fr) using a multiple protein sequence alignment produced with Clustal Omega, with secondary structure information extracted from the SmTopoI_N65 structure. ${ }^{119,120}$ Residues within $5 \AA$ of the active site Y316 were determined with UCSF Chimera and the structure distances measurement tool. ${ }^{118}$

\section{Structural Alignment of SmTopoI_N65 and TmTopoI (PDB 2GUI)}

Structural alignment of SmTopoI_N65 with TmTopoI (PDB 2GUI) conducted with UCSF Chimera MatchMaker structure comparison tool. ${ }^{101,118}$ The unique loop extension near the active site of SmTopoI_N65 is highlighted with a red circle.

\section{SmTopoI_N65 Sequence Alignment with EcTopoIII (PDB 1D6M)}

SmTopoI_N65 and EcTopoIII (PDB 1D6M) sequence alignment figure was generated with ESPript 3 server (http://espript.ibcp.fr) using multiple protein sequence alignment from Clustal Omega and secondary structure information from SmTopoI_N65 structure. ${ }^{119,122}$

\section{Results}

\section{Cloning and Expression of Recombinant SmTopoI}

Considering the need to rapidly isolate SmTopoI from host E. coli topoisomerase I, cloning into a poly-histidine affinity tag system was chosen and implemented. The $S$. mutans topA gene was first cloned and inserted into the $\mathrm{pET} 15 \mathrm{~b}$ vector (SmTopA15b), introducing a hexa-Histidine (hexa-His) tag at the $\mathrm{N}$-terminus of the target enzyme. ${ }^{123}$ The target gene was also cloned or sub-cloned into several alternate vectors in order to analyze the effects of different constructs, including affinity tag location and size, as well as the usefulness of a protein fusion tag. BL21(DE3)-Gold Competent cells were used for the over-expression of target protein. The target gene was also transformed into the ArcticExpress Competent Cell system to evaluate the utility of expressing at lower temperatures $\left(12^{\circ} \mathrm{C}\right)$ in order to increase solubility, but was not found to significantly improve results in this instance (data not shown). ${ }^{124,125}$

Preliminary mini-expression results from the SmTopI15b construct led to a traditional IPTG induction and expression that was implemented across all constructs in duplicate. Expression products were then carried into initial IMAC nickel column 
purification trials using a standard linear imidazole elution protocol. While SmTopI16b from the N-term deca-His construct and SmTopI-SUMO both significantly outperformed the others, SmTopI16b was ultimately selected and carried forward (Table 2-3). Of note, as the SmTopI-SUMO enzyme approached the customary $100-\mathrm{kDa}$ E. coli expression cutoff, the additional $11 \mathrm{kDa}$ in size added by the SUMO fusion tag showed no apparent deleterious effect on the expression in this respective system, demonstrated by the average calculated amounts of expressed recombinant protein from the construct trials resulting in $88.9 \mathrm{pmol}$ for the $\sim 83 \mathrm{kDa}$ SmTopI16b and $100 \mathrm{pmol}$ for the $\sim 93 \mathrm{kDa}$ SmTopI-SUMO, respectively.

As further loss of target protein was anticipated with additional purification steps, different conditions were also employed in order to further increase target protein expression and convenience, including the implementation of auto-induction for protein expression. ${ }^{106}$ Upon transitioning from a classical IPTG-induction method to a standard auto-induction method, the yield of SmTopI16b increased dramatically, as did the convenience of the protocol (Table 2-3). ${ }^{106}$ A subsequent IPTG mini-expression profile of SmTopI16b was also conducted in order to determine favorable IPTG expression conditions, which was also compared to auto-induced expression and illustrated no apparent loss of solubility from adopting an auto-induction method (Figure 2-2).

\section{Purification of Recombinant SmTopoI}

Early exploratory nickel-nitrilotriacetic acid $\left(\mathrm{Ni}^{2+}-\mathrm{NTA}\right)$ column IMAC purification with the $\mathrm{pET} 15 \mathrm{~b} \mathrm{~N}$-term hexa-His tag system showed the affinity of the tagged target protein for the $\mathrm{Ni}^{2+}$-NTA column to be markedly weak, leading to both an unacceptable loss of target protein during wash steps and very early dissociation upon elution. Furthermore, it was discovered upon peptide fingerprinting analysis that what was initially presumed to be the $\sim 83 \mathrm{kDa}$ SmTopI $15 \mathrm{~b}$ target protein upon SDS-PAGE analysis was actually the $\sim 84 \mathrm{kDa}$ native $E$. coli catalase HPII protein co-eluting with the target protein (Figure 2-3).

Comparative analysis of chromatographic linear elution profiles from the SmTopoI IMAC purification trials showed a slight increase in the retention time for the deca-His tagged target protein relative to the hexa-His tags (Figure 2-4). Therefore, the precise point of elution of SmTopI16b from the linear gradient was observed to be at $25 \%$ elution buffer (about $90 \mathrm{mM}$ imidazole), and this aspect was used to develop a step elution protocol. Upon implementation of a 15\%, 20\%, 100\% step elution protocol, SmTopI16b eluted in a sharp peak at the $100 \%$ elution step and was successfully separated from contaminating proteins, including E. coli catalase HPII, as determined by SDS-PAGE analysis and His-staining (Figure 2-3). As such, the pET16b N-term decaHis tag system was deemed the most successful and carried forward for further experimentation.

While analyzing mini-expression data, it was determined that target protein solubility could likely be improved. A simple transition from $50 \mathrm{mM}$ Tris- $\mathrm{HCl} \mathrm{pH} 8.0$ to 
Table 2-3. Comparative summary of purification yields from SmTopoI constructs.

\begin{tabular}{|c|c|c|c|}
\hline $\begin{array}{l}\text { Vector } \\
\text { System }\end{array}$ & $\begin{array}{c}\text { System } \\
\text { Characteristics }\end{array}$ & Growth/Induction Method & $\begin{array}{c}\text { Overall Yield } \\
(\mathrm{mg} / 500 \mathrm{~mL})^{a}\end{array}$ \\
\hline pET-15b & N-term $6 \mathrm{X}$ His tag & $\begin{array}{c}500 \mathrm{~mL} \text { TB; } 0.5 \mathrm{mM} \text { IPTG; } \\
18^{\circ} \mathrm{C} \text { x } 24 \mathrm{~h}\end{array}$ & 4.94 (IMAC) \\
\hline pET-16b & N-term 10X His tag & $\begin{array}{c}500 \mathrm{~mL} \mathrm{~TB} ; 0.5 \mathrm{mM} \text { IPTG; } \\
18^{\circ} \mathrm{C} \times 24 \mathrm{~h}\end{array}$ & $\begin{array}{l}7.11(\mathrm{IMAC}) \\
6.24(\mathrm{SE})\end{array}$ \\
\hline pET-21d & C-term 6X His tag & $\begin{array}{c}500 \mathrm{~mL} \mathrm{~TB} ; 0.5 \mathrm{mM} \text { IPTG; } \\
18^{\circ} \mathrm{C} \times 24 \mathrm{~h}\end{array}$ & 4.11 (IMAC) \\
\hline SUMO & $\begin{array}{l}\text { N-term SUMO fusion } \\
\text { tag and N-term } 6 \text { X His } \\
\text { tag }\end{array}$ & $\begin{array}{c}500 \mathrm{~mL} \text { TB; } 0.5 \mathrm{mM} \text { IPTG; } \\
18^{\circ} \mathrm{C} \times 24 \mathrm{~h}\end{array}$ & 9.12 (IMAC) \\
\hline pET-16b & N-term 10X His tag & $\begin{array}{c}500 \text { mL TB; } 0.5 \text { mM IPTG; } \\
18^{\circ} \mathrm{C} \text { x } 24 \text { h; high salt }\end{array}$ & 8.30 (IMAC) \\
\hline pET-16b & N-term 10X His tag & $\begin{array}{l}500 \mathrm{~mL} \text { auto-induction media; } \\
37^{\circ} \mathrm{C} \times 24 \mathrm{~h} \text {; high salt }\end{array}$ & $\begin{array}{c}14.21(\mathrm{IMAC}) \\
11.66(\mathrm{SE})\end{array}$ \\
\hline
\end{tabular}

${ }^{a}$ Overall yields are an average of two $500 \mathrm{~mL}$ growths in respective media.

Reprinted from final submission with permission from Elsevier Inc. Jones, J. A., Price, E., Miller, D. \& Hevener, K. E. A simplified protocol for high-yield expression and purification of bacterial topoisomerase I. Protein Expr Purif 124, 32-40, https://doi.org/10.1016/j.pep.2016.04.010 (2016). ${ }^{73}$ 


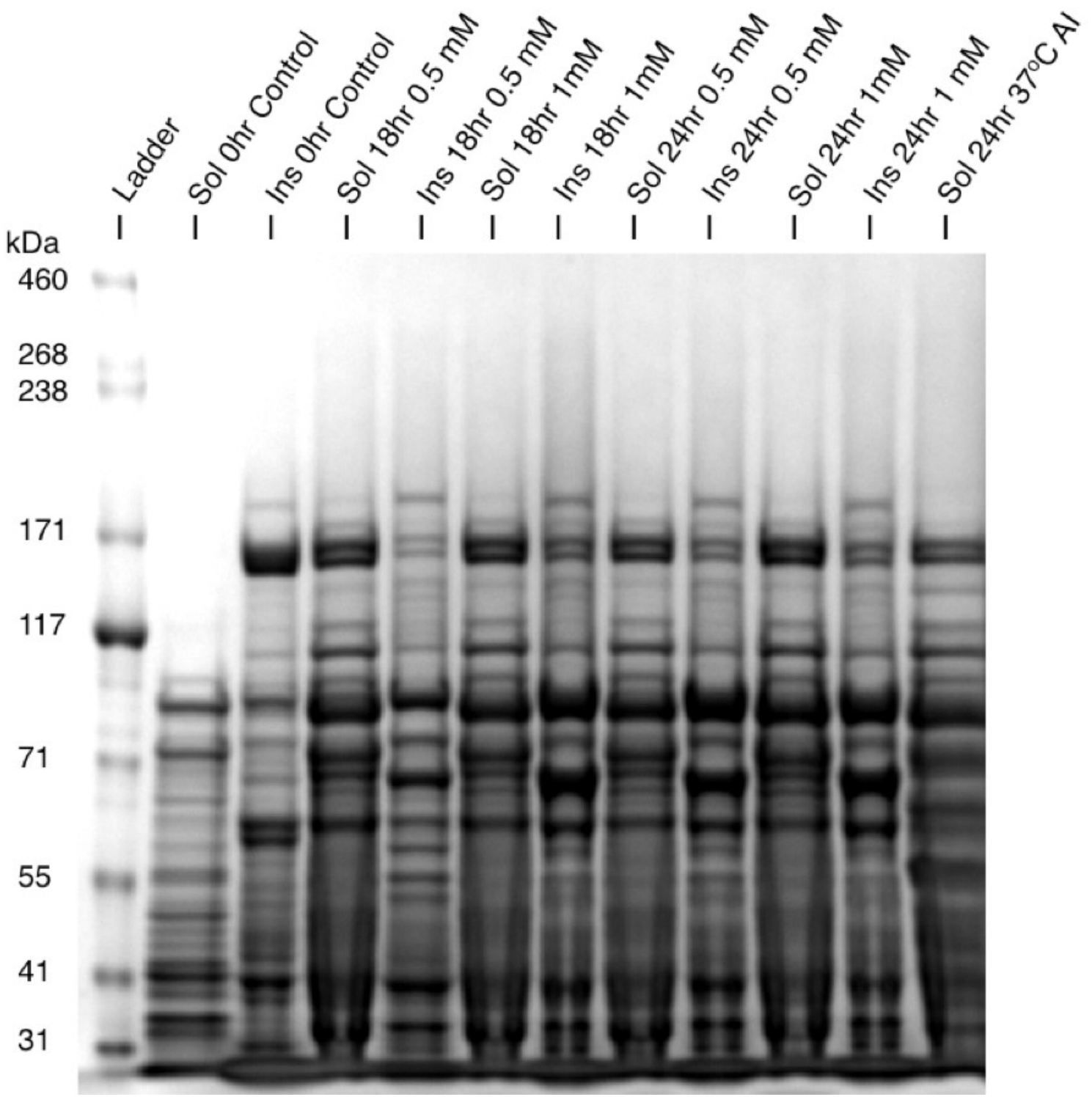

Figure 2-2. Mini-expression SDS-PAGE.

Mini-expression of SmTopI16b comparing $0.5 \mathrm{mM}$ and $1 \mathrm{mM}$ IPTG induction at $18^{\circ} \mathrm{C}$ over different times as well as auto-induction at $37^{\circ} \mathrm{C}$ over 24 hours (Sol, soluble fraction; Ins, insoluble fraction; AI, auto-induction). Insoluble auto-induction fraction not shown as it repeatedly resulted in an unacceptably high amount of smearing, presumed to be due to substantial pellet size and an excess of cellular debris.

Reprinted from final submission with permission of Elsevier Inc. Jones, J. A., Price, E., Miller, D. \& Hevener, K. E. A simplified protocol for high-yield expression and purification of bacterial topoisomerase I. Protein Expr Purif 124, 32-40, https://dx.doi.org/10.1016\%2Fj.pep.2016.04.010 (2016). ${ }^{73}$ 


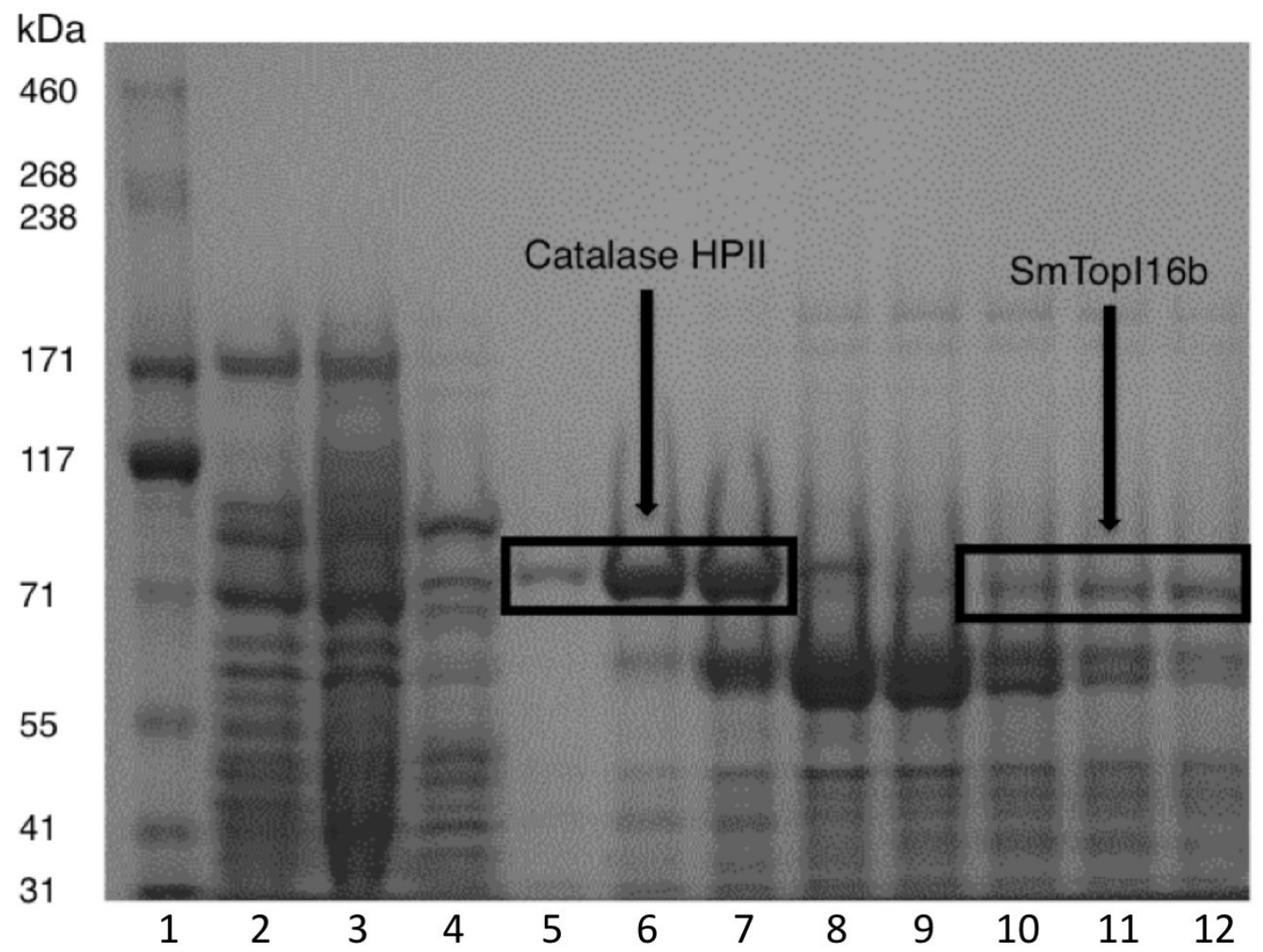

Figure 2-3. Catalase contaminant SDS PAGE.

SDS-PAGE showing initial difficulty presented by native catalase HPII contaminant at $\sim 84 \mathrm{kDa}$ and target enzyme at $\sim 83 \mathrm{kDa}$ during purification of $\mathrm{N}$-term hexa-His-tagged SmTopI_15b construct. Lane 1, ladder; 2-4, nickel column flow-through; 5-7, early nickel elution peak; 8-9, middle nickel elution peak; 10-12, tail end shoulder of nickel elution peak.

Reprinted from final submission with permission of Elsevier Inc. Jones, J. A., Price, E., Miller, D. \& Hevener, K. E. A simplified protocol for high-yield expression and purification of bacterial topoisomerase I. Protein Expr Purif 124, 32-40, https://dx.doi.org/10.1016\%2Fj.pep.2016.04.010 (2016). ${ }^{73}$ 

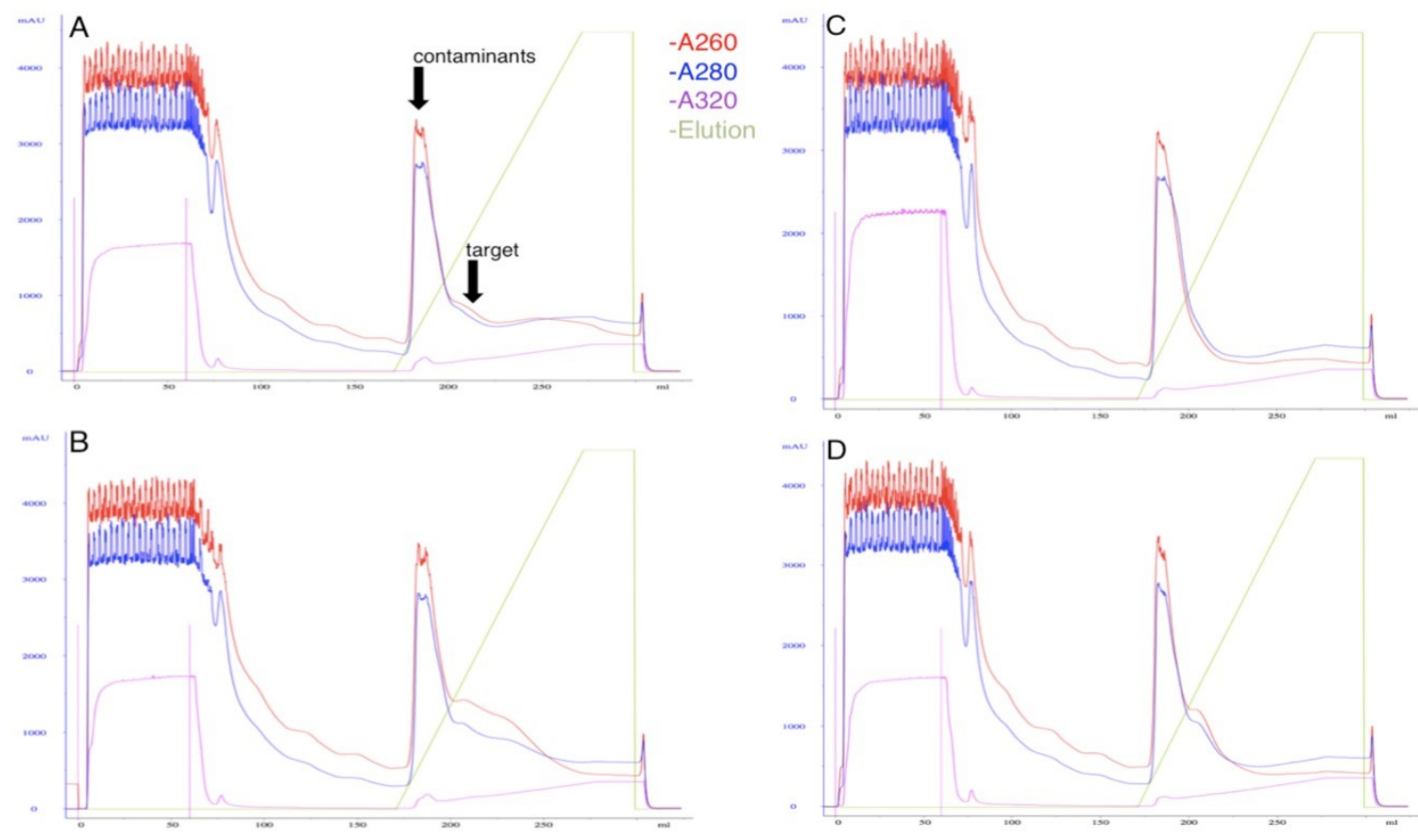

Figure 2-4. Chromatogram of nickel column linear elution from preliminary construct trials.

Direct vertical comparison. (A) N-term hexa-His-tagged SmTopI_15b construct. (B) Nterm deca-His-tagged SmTopI_16b construct. (C) C-term hexa-His-tagged SmTopI_21d construct. (D) N-term SUMO and hexa- his-tagged SUMO construct.

Reprinted from final submission with permission of Elsevier Inc. Jones, J. A., Price, E., Miller, D. \& Hevener, K. E. A simplified protocol for high-yield expression and purification of bacterial topoisomerase I. Protein Expr Purif 124, 32-40, https://dx.doi.org/10.1016\%2Fj.pep.2016.04.010. (2016). ${ }^{73}$ 
$20 \mathrm{mM}$ phosphate buffer $\mathrm{pH} 7.4$ in the lysis and nickel column buffers showed an apparent increase in yield and increasing the ionic strength of the nickel column binding and wash buffers (from $150 \mathrm{mM}$ to $1 \mathrm{M} \mathrm{NaCl}$ ) and the nickel column elution buffer (from $150 \mathrm{mM}$ to $500 \mathrm{mM} \mathrm{NaCl}$ ) greatly increased the overall purification yield of SmTopI16b (Table 2-3 and Figure 2-5). A long wash step over $40 \mathrm{CVs}$ with the higher ionic strength binding buffer also resulted in satisfactory removal of DNA from the target protein. Absorbance at $260 \mathrm{~nm}$ and $280 \mathrm{~nm}$ was monitored, ensuring the maximal removal of DNA. As stated above, transitioning to an auto-induction expression protocol also significantly improved yield and convenience.

Following successful IMAC purification, SmTopI16b was identified upon SDSPAGE analysis as an overexpressed band (Figure 2-5a). Target protein identity was confirmed initially using mass spectrometry peptide fingerprinting (Figure 2-6), secondarily upon His-epitope tag antibody directed western blot (data not shown), and again using His-tag staining (Figure 2-5b). Protein concentrations and yield were calculated using the standard Bradford protein assay with BSA as the protein standard, which is compatible with the IMAC elution buffer. ${ }^{126}$

Following optimization of the IMAC purification step, analyses with several alternative secondary purification methods, including the use of a heparin column, cation and anion exchange columns, a Cibacron Blue column, and a size exclusion (SE) column were conducted. Ultimately, transitioning straight from IMAC to a second, polishing size exclusion step was chosen due to simplicity and the fact that it showed the overall greatest yield. The target protein size of $\sim 83 \mathrm{kDa}$ was judged to be distinct enough from any of the remaining $\sim 97 \mathrm{kDa}$ native $E$. coli topoisomerase I to separate them using size exclusion chromatography. After increasing the ionic strength of the size exclusion buffer (from $150 \mathrm{mM} \mathrm{NaCl}$ to $500 \mathrm{mM} \mathrm{NaCl}$ ), the target protein solubility was found to be sufficient for purification without the need for an intermediate buffer exchange or dialysis step of the $\mathrm{Ni}^{2+}$-NTA purification product, which again afforded an additional gain in speed and convenience. Following the size exclusion chromatography polishing step, the target protein was collected and again analyzed via SDS-PAGE, indicating purity deemed greater than $95 \%$. Yield was again determined from duplicate trials by Bradford protein assay with BSA as a standard, which indicated over $23 \mathrm{mg} / \mathrm{L}$ of target protein (Table 2-3 and Table 2-4).

\section{Characterization of SmTopoI}

In order to track protein activity, a gel-based DNA-relaxation assay was employed using published methods (Figure 2-7). ${ }^{104}$ The activity of SmTopI16b was maintained over three months in SE Buffer 2 refrigerated at $4^{\circ} \mathrm{C}$, as well as after overnight freeze trials at $-80^{\circ} \mathrm{C}$ including enzyme with and without $50 \%$ glycerol using both snap-freezing and drop-wise freezing methods in liquid nitrogen (Figure 2-8). Protein activity after cleavage of the deca-His tag was also analyzed, showing no apparent increase in activity (Figure 2-9). Furthermore, the absence of DNase activity was determined via the 


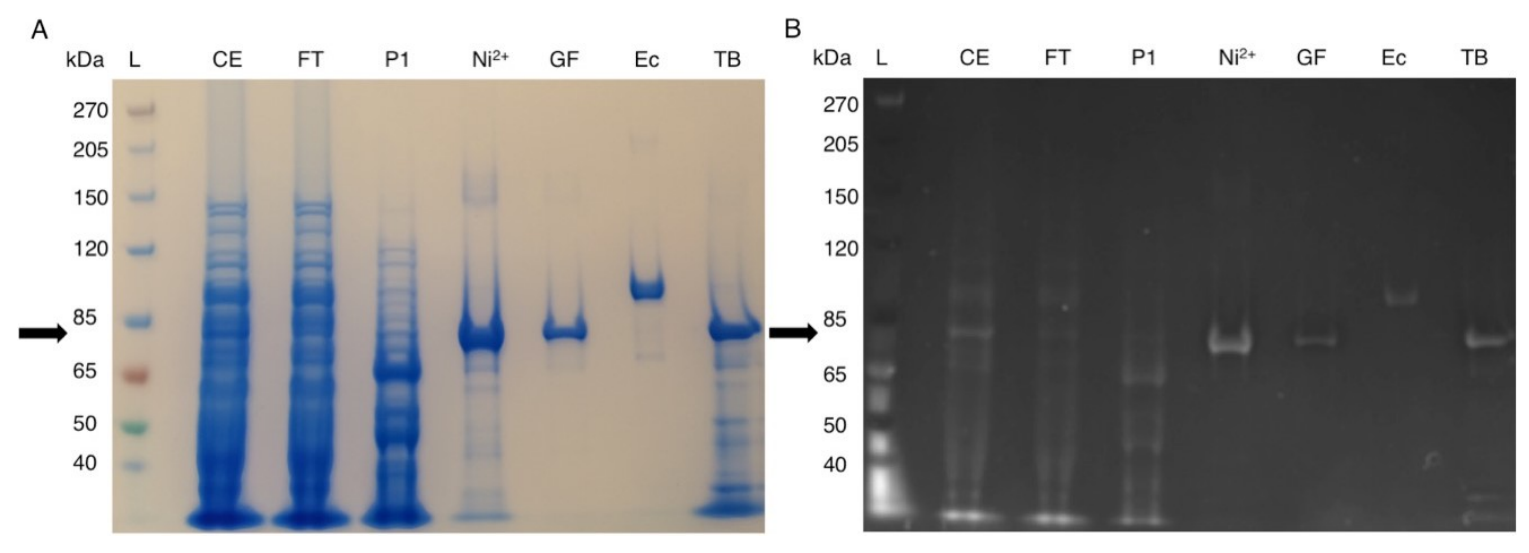

Figure 2-5. SDS-PAGE gels of SmTopI_16b purification results.

(A) SDS-PAGE after blue-staining. (B) SDS-PAGE after His-staining in order to verify target protein bands. L, ladder; CE, clarified extract from auto-induction growth; FT, nickel column flow-through; P1, nickel column first peak from first step elution using high salt buffers; Ni2+, target fraction from nickel column; SE, target fraction from size exclusion; Ec; commercially available E. coli topoisomerase I; TB, target fraction from nickel column from TB growth with high salt buffers.

Reprinted from final submission with permission of Elsevier Inc. Jones, J. A., Price, E., Miller, D. \& Hevener, K. E. A simplified protocol for high-yield expression and purification of bacterial topoisomerase I. Protein Expr Purif 124, 32-40, https://dx.doi.org/10.1016\%2Fj.pep.2016.04.010. (2016). ${ }^{73}$ 

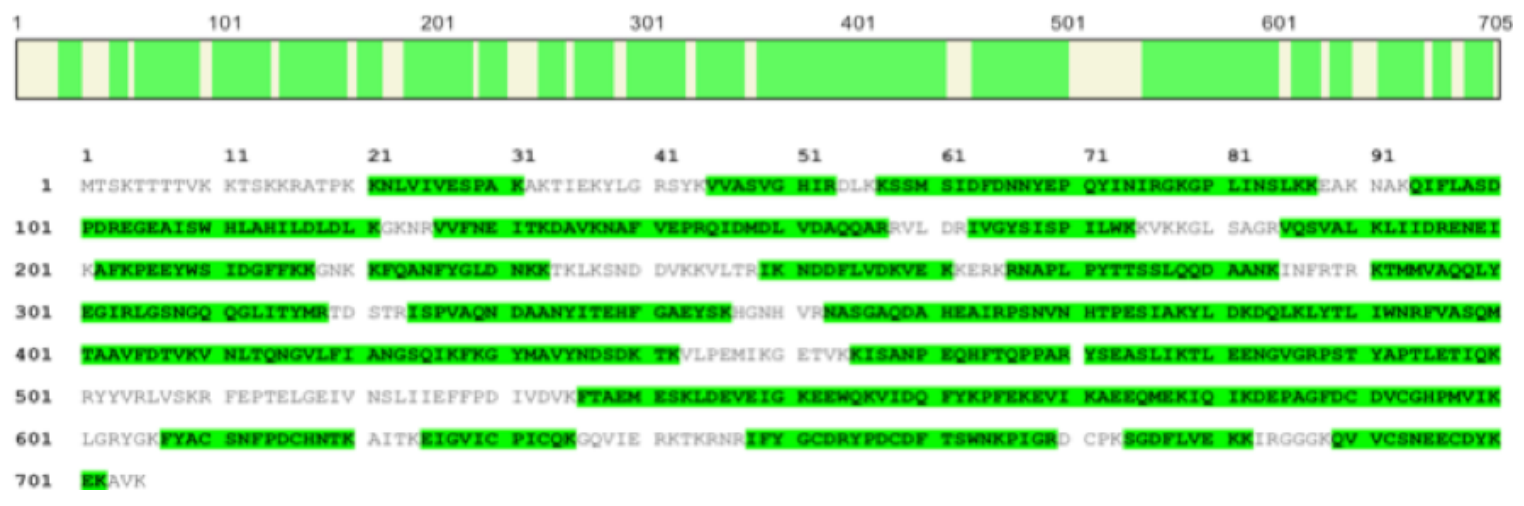

\section{Figure 2-6. Peptide fingerprinting.}

Peptide fingerprinting analysis showing $74.20 \%$ sequence coverage for Topoisomerase I from Streptococcus mutans serotype c (strain ATCC 700610/UA159).

Reprinted from final submission with permission of Elsevier Inc. Jones, J. A., Price, E., Miller, D. \& Hevener, K. E. A simplified protocol for high-yield expression and purification of bacterial topoisomerase I. Protein Expr Purif 124, 32-40, https://dx.doi.org/10.1016\%2Fj.pep.2016.04.010. (2016). ${ }^{73}$

Table 2-4. Summary of S. mutans topoisomerase I purification.

\begin{tabular}{lcccccc}
\hline Step & $\begin{array}{c}\text { Total } \\
\text { Protein } \\
(\mathbf{m g})^{\mathbf{a}}\end{array}$ & $\begin{array}{c}\text { Target } \\
\text { Protein } \\
(\mathbf{m g})^{\mathbf{b}}\end{array}$ & $\begin{array}{c}\text { Total } \\
\text { Activity } \\
\text { (units) }^{\mathbf{c}}\end{array}$ & $\begin{array}{c}\text { Specific } \\
\text { Activity } \\
\text { (units/mg) }\end{array}$ & $\begin{array}{c}\text { Yield } \\
(\%)\end{array}$ & $\begin{array}{c}\text { Purity } \\
\text { (\%) }^{\mathbf{d}}\end{array}$ \\
\hline $\begin{array}{l}\text { Clarified } \\
\text { extract }\end{array}$ & 1445 & 107.3 & N/A & N/A & 100 & 7.4 \\
$\begin{array}{l}\text { Nickel } \\
\text { column }\end{array}$ & 28.4 & 25.3 & N/A & N/A & 24 & 89 \\
$\begin{array}{l}\text { Size } \\
\text { exclusion } \\
\text { column }\end{array}$ & 23.3 & 22.6 & 58,250 & 2,575 & 21 & 97 \\
\hline
\end{tabular}

${ }^{a}$ Protein concentration determined by Bradford assay using BSA as the standard protein.

${ }^{b}$ Amount of target protein determined based off of SDS-PAGE gel analysis.

${ }^{c}$ Activity measured as described under Materials and Methods section.

${ }^{\mathrm{d}}$ Purity determined based off of SDS-PAGE gel analysis.

Reprinted from final submission with permission from Elsevier Inc. Jones, J. A., Price, E., Miller, D. \& Hevener, K. E. A simplified protocol for high-yield expression and purification of bacterial topoisomerase I. Protein Expr Purif 124, 32-40, https://doi/org.10.1016/j.pep.2016.04.010 (2016). ${ }^{73}$ 


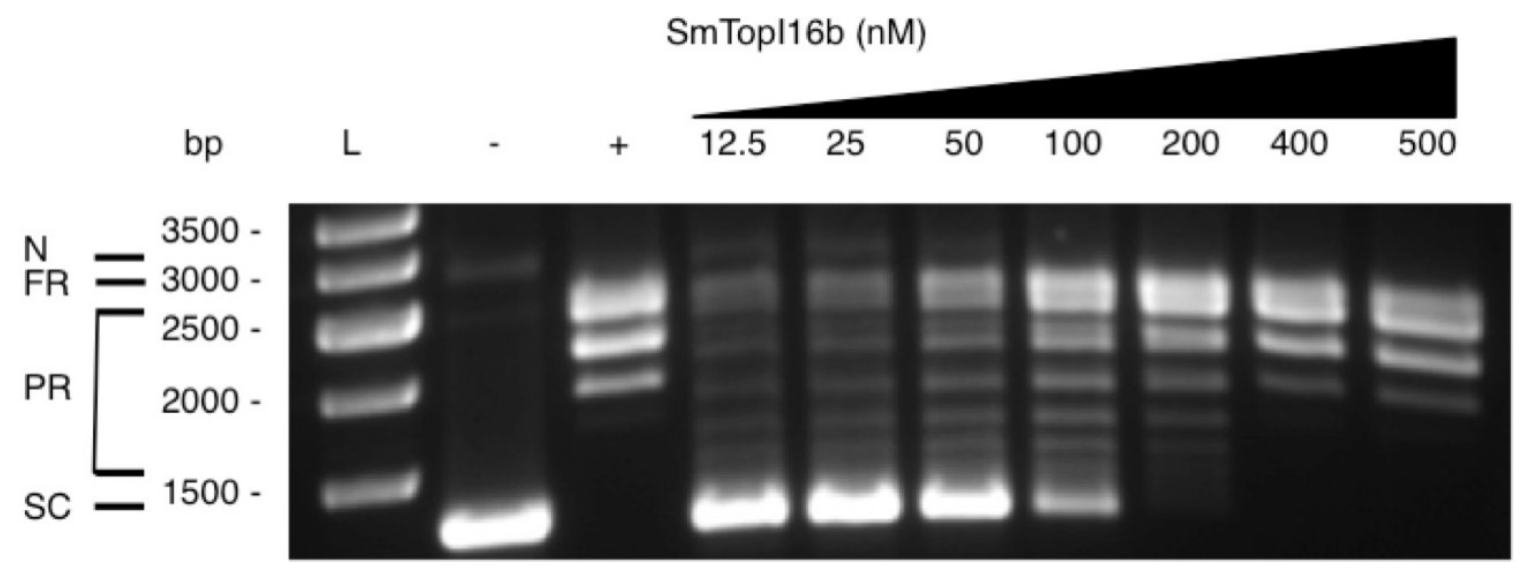

Figure 2-7. Gel-based DNA relaxation assay.

Lane 1, ladder; lane 2, supercoiled pUC18 plasmid (DNA substrate as negative control); lane 3, commercially available E. coli topoisomerase I (positive control); lanes 4-10, SmTopI16b enzyme in increasing concentration (nM). N, nicked; FR, fully relaxed; PR, partially relaxed; SC, supercoiled.

Reprinted from final submission with permission of Elsevier Inc. Jones, J. A., Price, E., Miller, D. \& Hevener, K. E. A simplified protocol for high-yield expression and purification of bacterial topoisomerase I. Protein Expr Purif 124, 32-40, https://dx.doi.org/10.1016\%2Fj.pep.2016.04.010. (2016). ${ }^{73}$ 


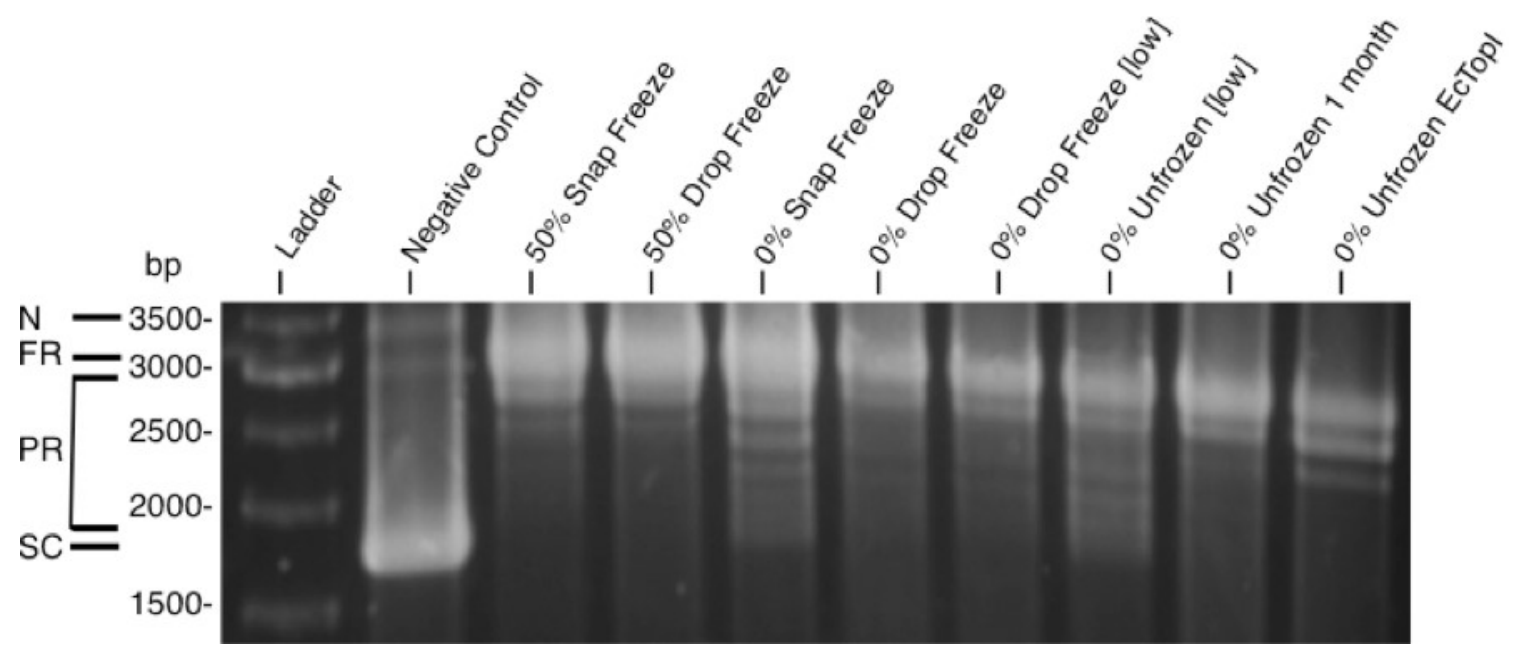

Figure 2-8. SmTopI16b freeze trials.

Agarose gel showing activity of target enzyme frozen via different methods ( 1 ug enzyme used, except 400ng used for [low] samples and 1 unit for EcTopoI positive control). Reprinted from final submission with permission of Elsevier Inc. Jones, J. A., Price, E., Miller, D. \& Hevener, K. E. A simplified protocol for high-yield expression and purification of bacterial topoisomerase I. Protein Expr Purif 124, 32-40, https://dx.doi.org/10.1016\%2Fj.pep.2016.04.010. (2016). ${ }^{73}$

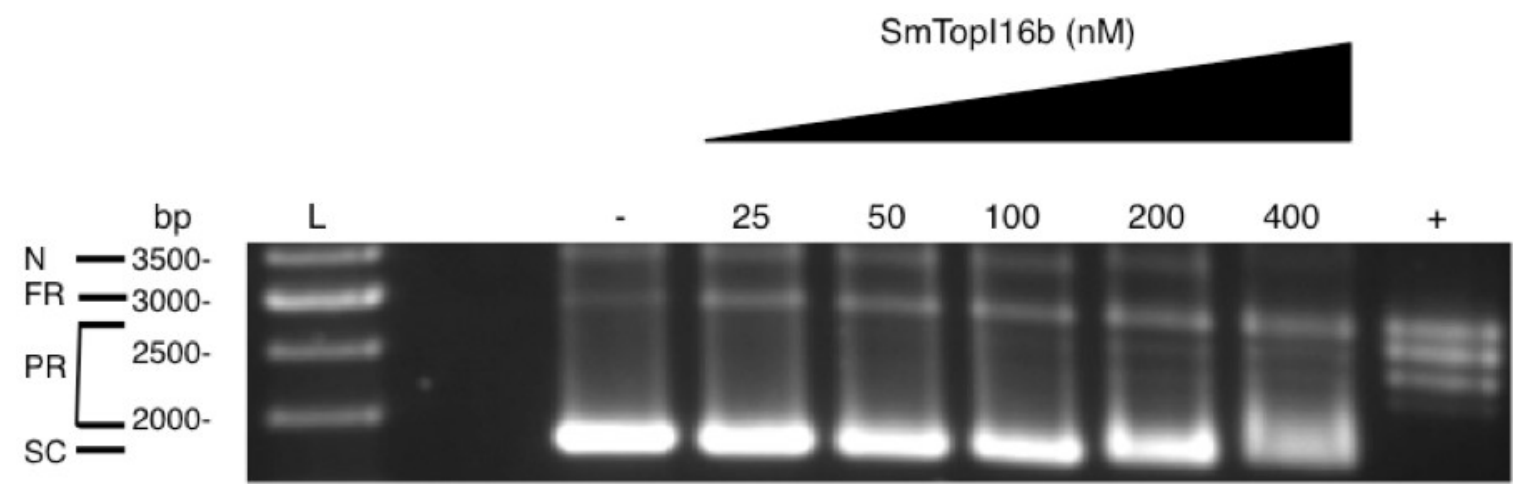

Figure 2-9. His-tag cleavage activity assay.

Agarose gel showing activity of SmTopI16b after cleavage of deca-his tag via factor Xa. Reprinted from final submission with permission of Elsevier Inc. Jones, J. A., Price, E., Miller, D. \& Hevener, K. E. A simplified protocol for high-yield expression and purification of bacterial topoisomerase I. Protein Expr Purif 124, 32-40, https://dx.doi.org/10.1016\%2Fj.pep.2016.04.010. (2016). ${ }^{73}$ 
implementation of a non-specific DNase activity assay using published methods (Figure 2-10). ${ }^{103}$

To characterize the stability of SmTopI16b, a number of methods were employed. Hampton Research Solubility \& Stability Screen $2^{\mathrm{TM}}$ was used to assess conditions that mitigate target protein precipitation and increase its overall stability. ${ }^{127}$ Optimal buffer conditions as determined via Differential Scanning Fluorimetry (DSF, or "thermal shift") from the screen were found to be $0.05 \mathrm{M}$ sodium citrate tribasic dihydrate $(\mathrm{pH} \mathrm{5.0)}$ with 4.0 M sodium chloride as showcased by an optimal melting temperature $(\mathrm{Tm})$ of $48^{\circ} \mathrm{C}$ (Figure 2-11). An apparent trend was seen showing optimal buffer conditions included relatively low $\mathrm{pH}$ and high salt content. Protein solubility was also analyzed via overnight dialysis trials into lower-salt-containing buffers across a wide range of $\mathrm{pH}$ values. Results showed considerable visual precipitation regardless of buffer and $\mathrm{pH}$ when ionic strength was decreased below $200 \mathrm{mM} \mathrm{NaCl}$ with protein concentration at $1 \mathrm{mg} / \mathrm{mL}$, indicating the necessity of a higher ionic strength buffer for the solubility of this particular enzyme and construct (data not shown).

\section{Crystallization of SmTopoI_N65}

As reported previously, soluble full-length SmTopoI did not initially express well and, even after expression was optimized, its solubility required extremely high salt conditions not generally conducive to crystallization. ${ }^{73}$ As such, full-length SmTopoI proved recalcitrant to crystallization. Eventually, the C-terminal domain was rationally removed for subsequent crystallization trials. Iteratively shorter $\mathrm{N}$-terminal fragment SmTopoI constructs corresponding to the original $67-\mathrm{kDa} \mathrm{N}$-terminal EcTopoI (EcTopoI_N67) crystal structure (PDB 1ECL) ${ }^{99,100}$ were cloned, expressed, purified, and screened for crystals. After multiple fragments were screened, this eventually proved successful as diffraction-quality crystals of the $65-\mathrm{kDa} 575$ residue SmTopoI fragment were attained, however the diffraction resolution was relatively low (greater than $4 \AA$ ). Upon fine grid screening and implementation of temperature trials, high-resolution quality crystals were grown (Figure 2-12), and the structure was determined (PDB 6OZW).

\section{Overall Structure of SmTopoI_N65}

The three-dimensional crystal structure of SmTopoI_N65 was determined to 2.06 $\AA$ via molecular replacement using the EcTopoI_N67 crystal structure (PDB 1ECL) as a model, ${ }^{99,100}$ followed by iterative cycles of model building and refinement (Table 2-5). A sequence alignment between SmTopoI_N65 and EcTopoI_N67, which share a 42.9\% sequence identity, is shown (Figure 2-13). The root mean square deviation (RMSD) between the two structures is $1.83 \AA$. Analysis of the crystal structure and Matthews Coefficient indicate that there is one monomeric SmTopoI_N65 molecule per asymmetric unit (Figure 2-14). ${ }^{128-130}$ The monomeric state of SmTopoI N65 was further corroborated by PISA (Protein, Interface, Structures, and Assemblies) analysis. ${ }^{131}$ 


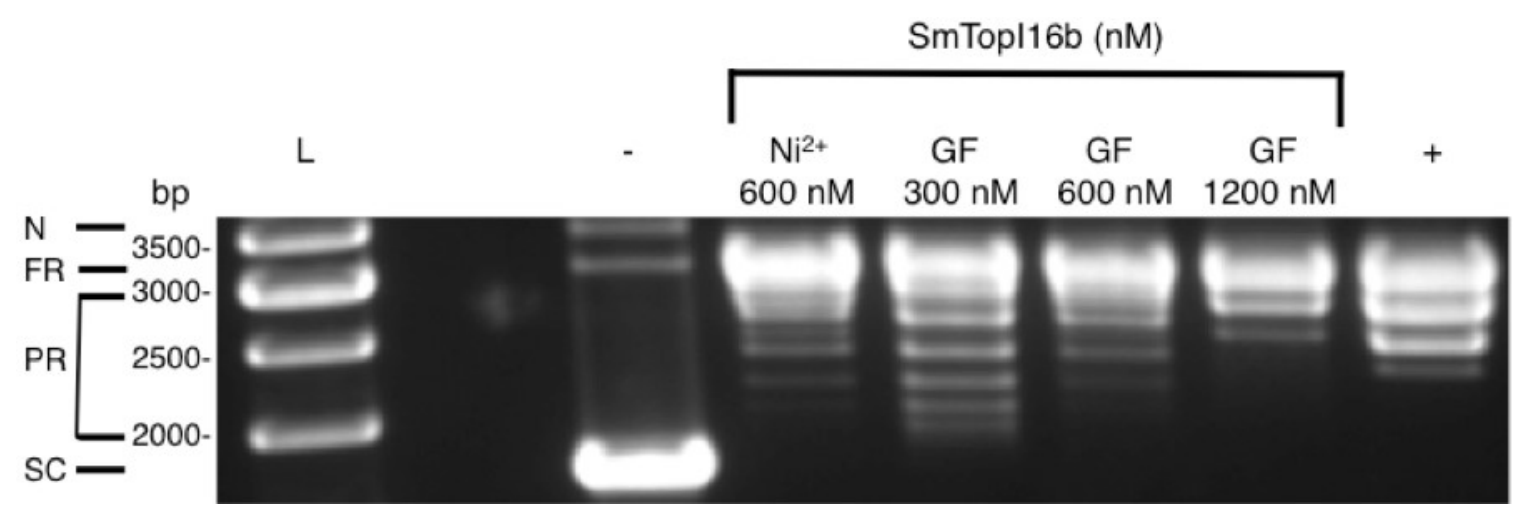

Figure 2-10. Non-Specific DNase Activity Assay.

Agarose gel confirming a lack of DNase activity after 6 hours incubation.

Reprinted from final submission with permission of Elsevier Inc. Jones, J. A., Price, E., Miller, D. \& Hevener, K. E. A simplified protocol for high-yield expression and purification of bacterial topoisomerase I. Protein Expr Purif 124, 32-40, https://dx.doi.org/10.1016\%2Fj.pep.2016.04.010. (2016). ${ }^{73}$ 


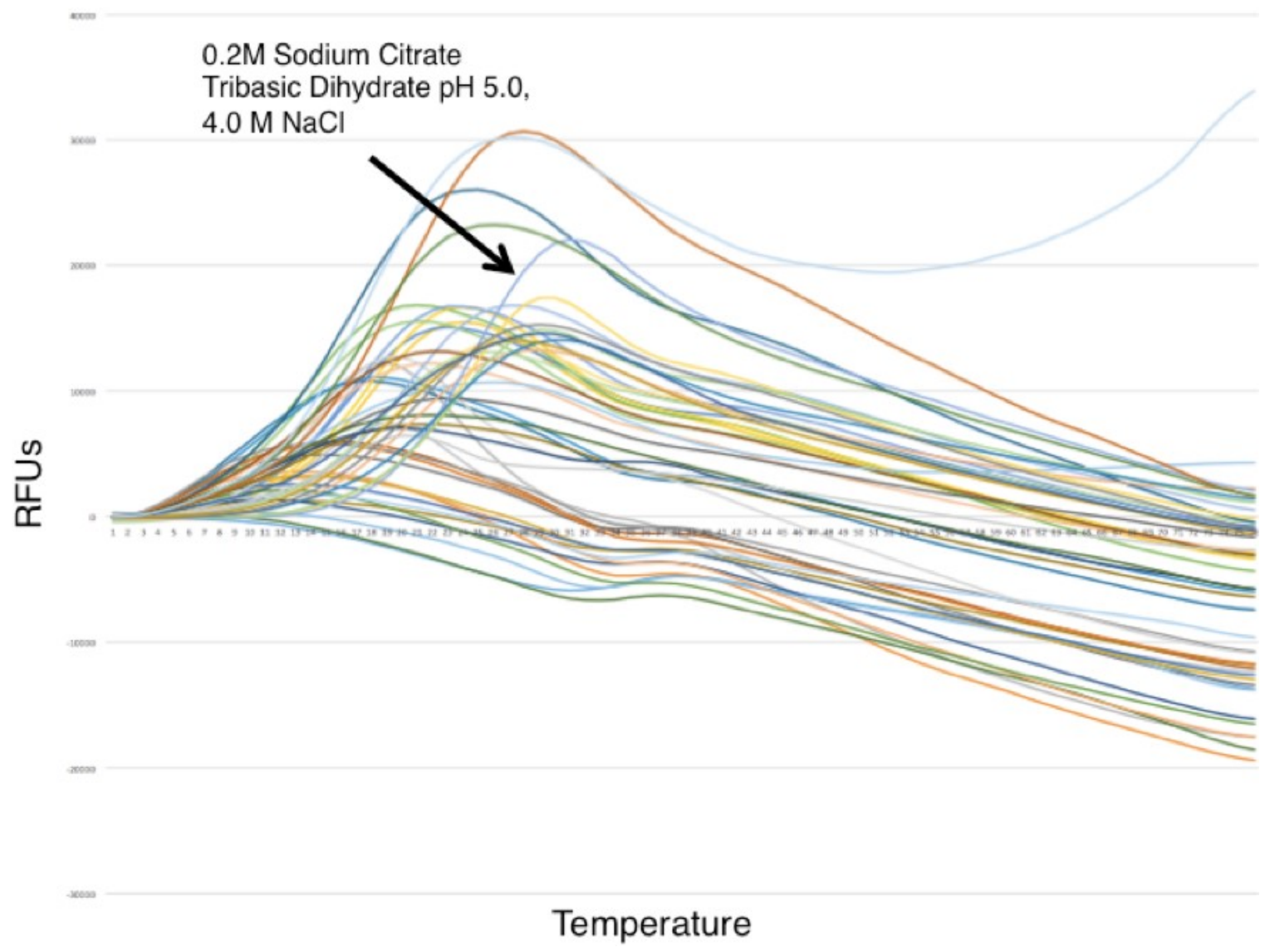

Figure 2-11. Thermal stability data.

Data showing differing thermal stability profiles of SmTopI16b with different salts and buffers.

Reprinted from final submission with permission of Elsevier Inc. Jones, J. A., Price, E., Miller, D. \& Hevener, K. E. A simplified protocol for high-yield expression and purification of bacterial topoisomerase I. Protein Expr Purif 124, 32-40, https://dx.doi.org/10.1016\%2Fj.pep.2016.04.010. (2016). ${ }^{73}$ 

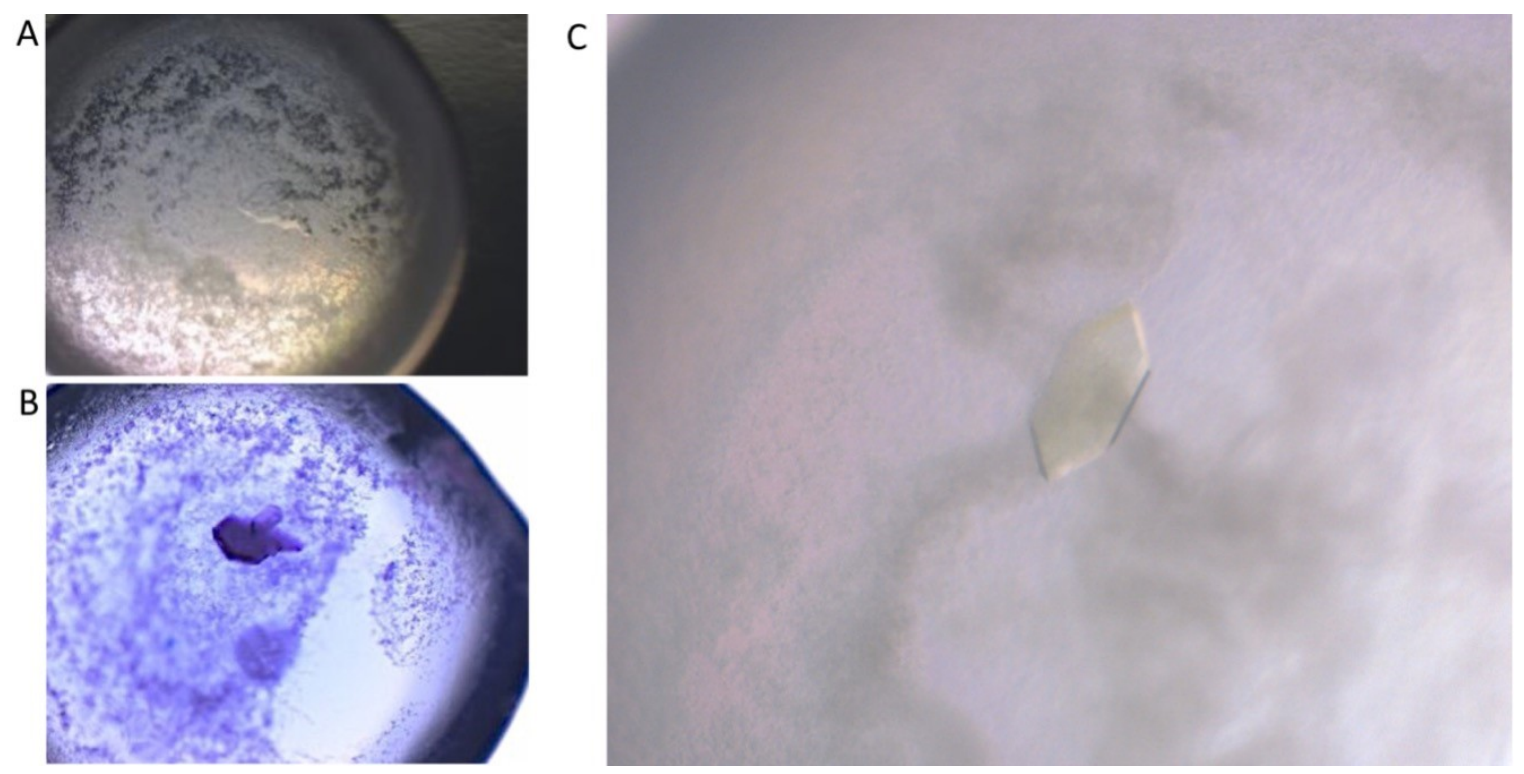

\section{Figure 2-12 SmTopoI_N65 crystals.}

A. Original SmTopoI_N65 crystals grown at $18^{\circ} \mathrm{C}$ from coarse JCSG plus screen, condition 1-5 (0.2 M magnesium formate dihydrate and 20\% PEG 3350). B. Same crystal as in (A), but stained with a 1:1 mixture of Izit Crystal Dye (Hampton Research, Aliso Viejo, CA) and acid red dye. C. Optimized SmTopoI_N65 crystal grown in 0.2 M magnesium formate dihydrate and $25 \%$ PEG 3350 at $4{ }^{\circ} \mathrm{C}$ used for structure determination.

Reprinted from final submission with open access permission, Elsevier, Inc. Jones, J. A. \& Hevener, K. E. Crystal structure of the 65-kilodalton amino-terminal fragment of DNA topoisomerase I from the gram-positive model organism Streptococcus mutans. Biochem Biophys Res Commun, https://doi.org/10.1016/j.bbrc.2019.06.034 (2019. In Press). ${ }^{74}$ 
Table 2-5. Data collection and refinement statistics for SmTopo_N65.

\begin{tabular}{|c|c|}
\hline Data Collection & Results \\
\hline Wavelength $(\AA)$ & 1.033 \\
\hline Space Group & P 21212 \\
\hline \multicolumn{2}{|l|}{ Unit cell parameters } \\
\hline$a, b, c(\AA)$ & 88.5898 .3772 .71 \\
\hline$\alpha, \beta, \gamma\left({ }^{\circ}\right)$ & 909090 \\
\hline Resolution (Å) & $50.00-2.06(2.10-2.06)^{\mathrm{a}}$ \\
\hline $\mathrm{CC}_{1 / 2}$ & $0.999(0.690)$ \\
\hline $\mathbf{R}_{\text {meas }}$ & $0.084(1.047)$ \\
\hline $\mathbf{R}_{\text {pim }}$ & $0.032(0.441)$ \\
\hline Total no. of reflections & 254919 \\
\hline No. of unique reflections & $32714(1394)$ \\
\hline Completeness $(\%)$ & $82.17(35.50)$ \\
\hline Redundancy & $6.5(5.1)$ \\
\hline Mean I/sigma & $21.8(1.5)$ \\
\hline \multicolumn{2}{|l|}{ Refinement } \\
\hline Number of reflections used & $32713(1395)$ \\
\hline Resolution (Å) & $49.18-2.063(2.137-2.063)$ \\
\hline $\mathbf{R}_{\text {work }}$ & $0.2003(0.2310)$ \\
\hline $\mathbf{R}_{\text {free }}$ & $0.2677(0.3617)$ \\
\hline No. of non-H atoms & 4700 \\
\hline Macromolecules & 4339 \\
\hline Ligands & 48 \\
\hline Solvent & 313 \\
\hline Protein residues & 538 \\
\hline RMS (bonds) & 0.008 \\
\hline RMS (angles) & 0.92 \\
\hline Ramachandran favored (\%) & 96.24 \\
\hline Ramachandran allowed (\%) & 3.20 \\
\hline Ramachandran outliers (\%) & 0.56 \\
\hline Rotamer outliers (\%) & 0.42 \\
\hline Clash score & 4.98 \\
\hline Average B-factor & 33.18 \\
\hline Macromolecules & 33.10 \\
\hline Ligands & 45.88 \\
\hline Solvent & 32.35 \\
\hline
\end{tabular}

${ }^{\text {a }}$ Statistics for the highest-resolution shell are shown in parentheses. $\mathrm{CC}_{1 / 2}$ is the Pearson's correlation coefficient of random half sets of data. Adapted from final submission with open access permission from Elsevier Inc. Jones, J. A. \& Hevener, K. E. Crystal structure of the 65-kilodalton amino-terminal fragment of DNA topoisomerase I from the gram-positive model organism Streptococcus mutans. Biochem Biophys Res Commun, https://doi/org.10.1016/j.bbrc.2019.06.034 (2019. In Press). ${ }^{74}$ 


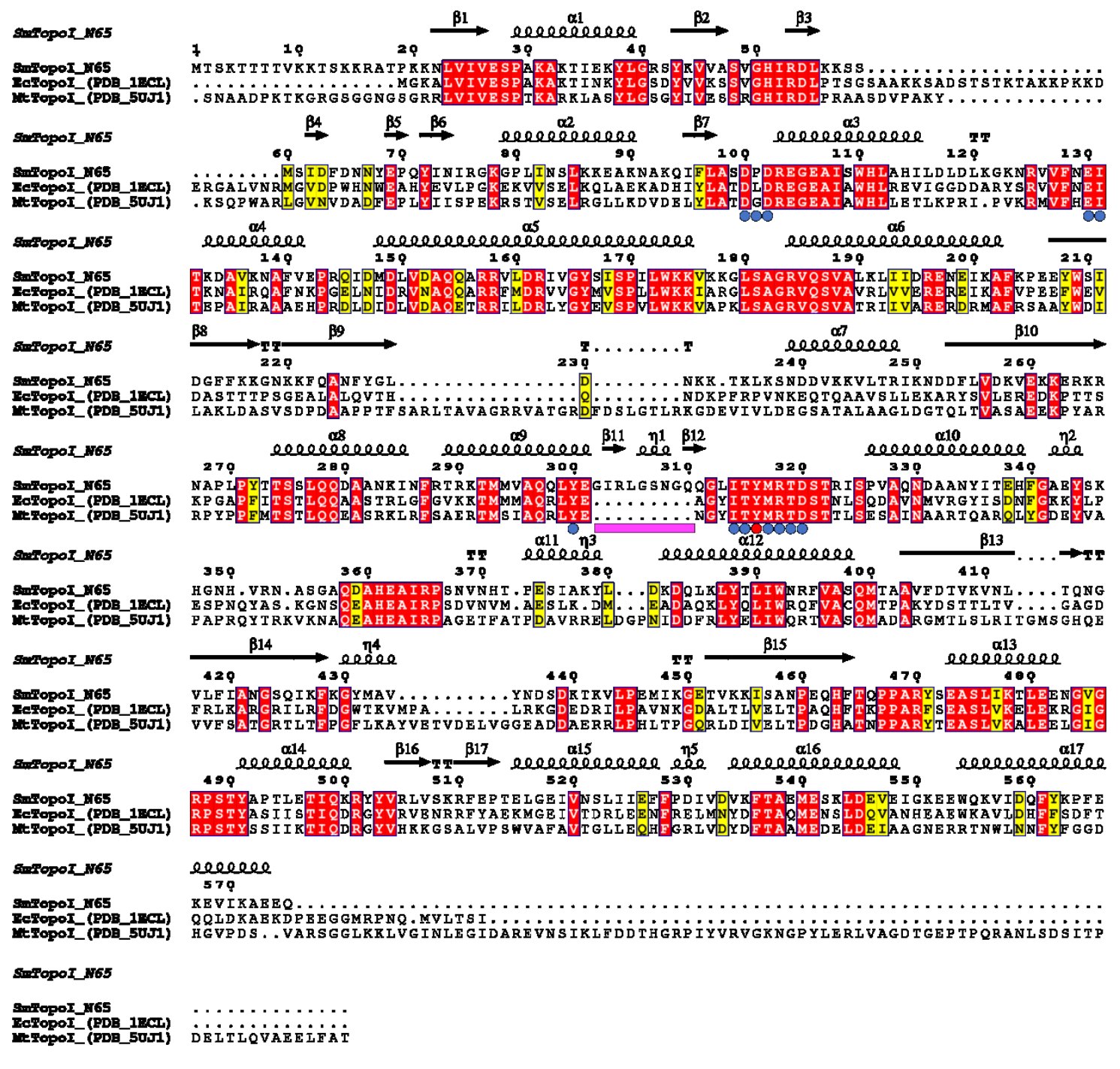

Figure 2-13. Multiple sequence alignment of SmTopoI_N65 and structural homologs.

Comparison of SmTopoI_N65, EcTopoI_N67, and MtTopoI via multiple sequence alignment shows high degree of sequence identity and similarity, including the highly conserved active site. Identical residues are highlighted in red, similar residues are highlighted in yellow, catalytic tyrosine is indicated with a red dot, active site residues within $5 \AA$ from catalytic tyrosine are indicated with blue dots, and the nine-residue loop extension seen in SmTopoI_N65 is indicated with a magenta bar. ${ }^{98,99,118}$ Reprinted from final submission with open access permission, Elsevier, Inc. Jones, J. A. \& Hevener, K. E. Crystal structure of the 65-kilodalton amino-terminal fragment of DNA topoisomerase I from the gram-positive model organism Streptococcus mutans. Biochem Biophys Res Commun, https://doi.org/10.1016/j.bbrc.2019.06.034 (2019. In Press). ${ }^{74}$ 

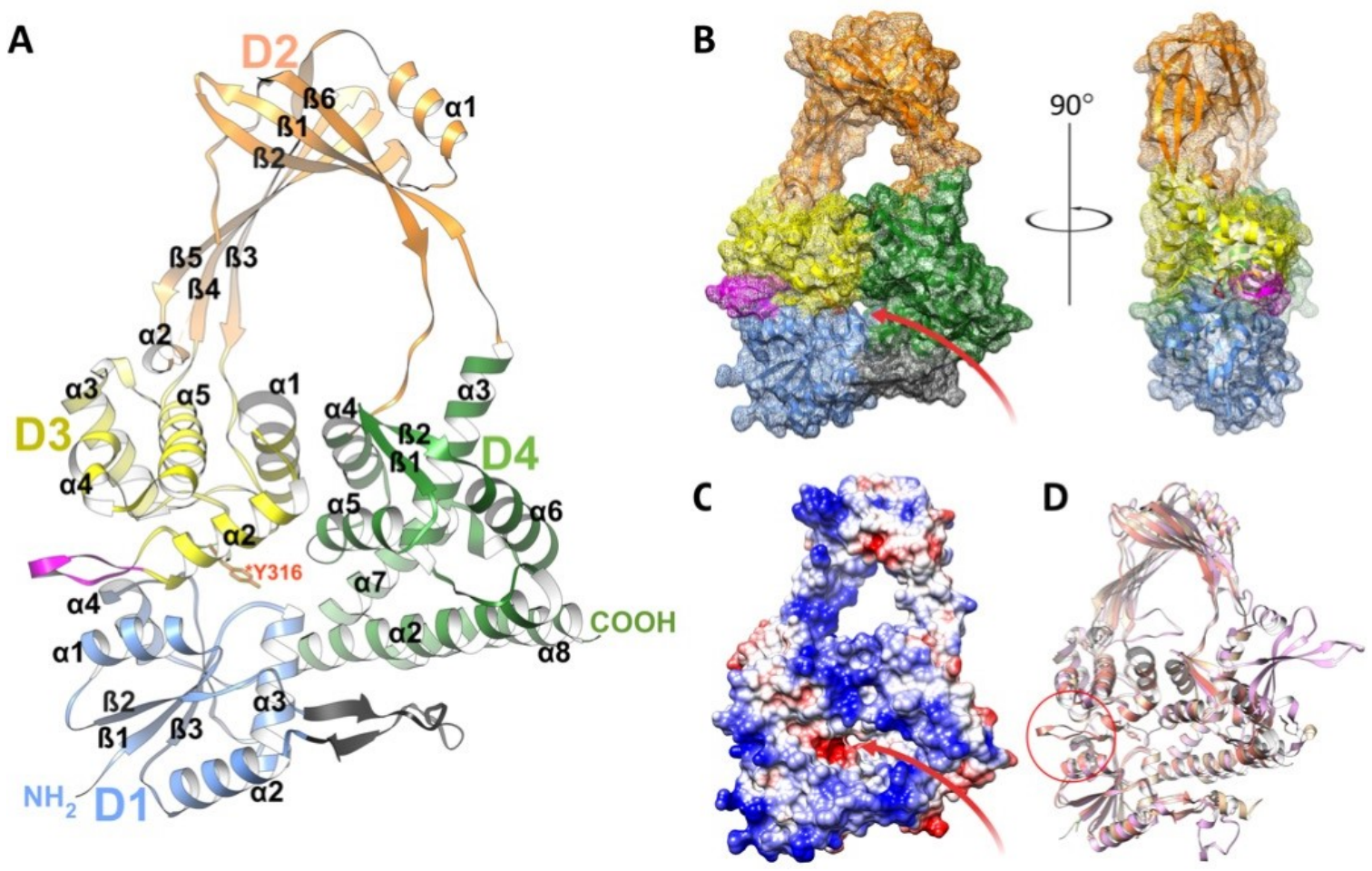

Figure 2-14. Overall structure of SmTopoI_N65

A. Flat ribbon diagram of the overall structure of SmTopoI_N65 showcasing the four Nterminal domains that comprise the main body. D1, D2, D3, and D4 are colored in blue, orange, yellow, and green, respectively. Sub-domain secondary structures are also numbered respectively. The unique loop extension before and immediately adjacent to the active site in D3 between $\alpha 2$ and $\alpha 3$ is colored magenta. The small outcropping from D1 that is considered a part of D4 as in the EcTopoI and MtTopoI structures is colored charcoal. The catalytic Y316 residue in D3 is drawn in stick and colored red. B. Mesh surface diagram of SmTopoI_N65 with the same color and orientation as in A, but turned on $\mathrm{x}$ axis by $-10^{\circ}$ for improved view of DNA binding site and groove, shown as a red arrow, with accompanying $90^{\circ}$ turn along the y axis for profile view. C. Coulombic surface coloring showing electrostatic potential with standard red/negative and blue/positive coloring in the same orientation as B. The DNA binding site and groove is also shown with a red arrow. D. Structural alignment of SmTopoI_N65 with EcTopoI_N67 (1ECL) and MtTopoI. ${ }^{118}$

Reprinted from final submission with open access permission, Elsevier Inc. Jones, J. A. \& Hevener, K. E. Crystal structure of the 65-kilodalton amino-terminal fragment of DNA topoisomerase I from the gram-positive model organism Streptococcus mutans. Biochem Biophys Res Commun, https://doi.org/10.1016/j.bbrc.2019.06.034 (2019. In Press). ${ }^{74}$ 
Electron density of the hexa-histidine tag is not visible in the model, nor is density for the first leading $18 \mathrm{~N}$-terminal residue peptide chain (M1 to T18), which exists as an additional N-term extension compared to EcTopoI, similar to the first 16 residues seen in MtTopoI (PDB 5UJ1). ${ }^{95}$ This unmodeled N-term peptide continues to be of unknown function. It carries substantial positive charge and is relatively polar, with five positively charged lysines and one positively charged arginine, as well as six polar uncharged threonines and two polar uncharged serines. The SmTopoI_N65 model begins at P19, with K21 of SmTopoI_N65 aligning with K3 of EcTopoI_N67. This represents the beginning of the highly conserved topoisomerase-primase (TOPRIM) domain, which ends at N129 and N140 of SmTopoI_N65 and EcTopoI_N67, respectively. ${ }^{132}$

The SmTopoI_N65 model includes the first four domains of the overall structure, which comprise the main body core and show an intricate topology (Figure 2-14 and Figure 2-15). ${ }^{133}$ Domains D1, D3, and D4 form the base of the body, and D2 forms the bulk of the toroid arch that represents the hinge domain. D1 is comprised of four alpha helices and three beta strands. D2 is connected to D4 via a bridge of two flexible loops and is comprised overall of two alpha helices and six beta strands. D3 is comprised of five alpha helices, with a likelihood of $\alpha 4$ actually forming a $3{ }_{10}$ helix. The catalytic Y316 residue is also located within D3 on a loop between $\alpha 2$ and $\alpha 3$ at the interface of D1, D3, and D4. The remainder of the highly conserved active site surrounds the catalytic tyrosine residue.

While the SmTopoI_N65 fragment shares 42.9\% sequence identity with the EcTopoI_N67 sequence and 25.19\% sequence identity with the EcTopoIII sequence (PDB 1D6M, Figure 2-16), full-length SmTopoI shares 41.4\% identity with full-length EcTopoI and 25.3\% with full-length $E$. coli topo III, 37.1\% identity with full-length MtTopoI, and $42.8 \%$ identity with full-length topo I from the thermophile T. maritima (TmTopoI). ${ }^{120}$ Of note, full-length SmTopoI shares $61.9 \%$ identity with the topo I enzyme from Staphylococcus aureus (SaTopoI), for which there is currently no determined three-dimensional structure.

\section{Discussion}

\section{Expression and Purification of SmTopoI}

An active full-length bacterial topoisomerase I enzyme has been purified to a relatively high yield and purity from the clinically relevant bacteria $S$. mutans. A systematic analysis of different protein constructs was necessary as an unacceptable amount of the prototype hexa-his tagged protein showed weak affinity for the nickel column and was therefore lost to flow-through and overly contaminated with early coeluting proteins. In order to analyze the possibility of the obstruction of the N-term His tag in the protein's folded state, the target gene was cloned into a pET21d vector (SmTopA21d), placing a hexa-His tag at the C-terminus. As increasing the length of histidine tags has been shown to increase affinity to $\mathrm{Ni}^{2+}$-NTA, allowing for the use of 


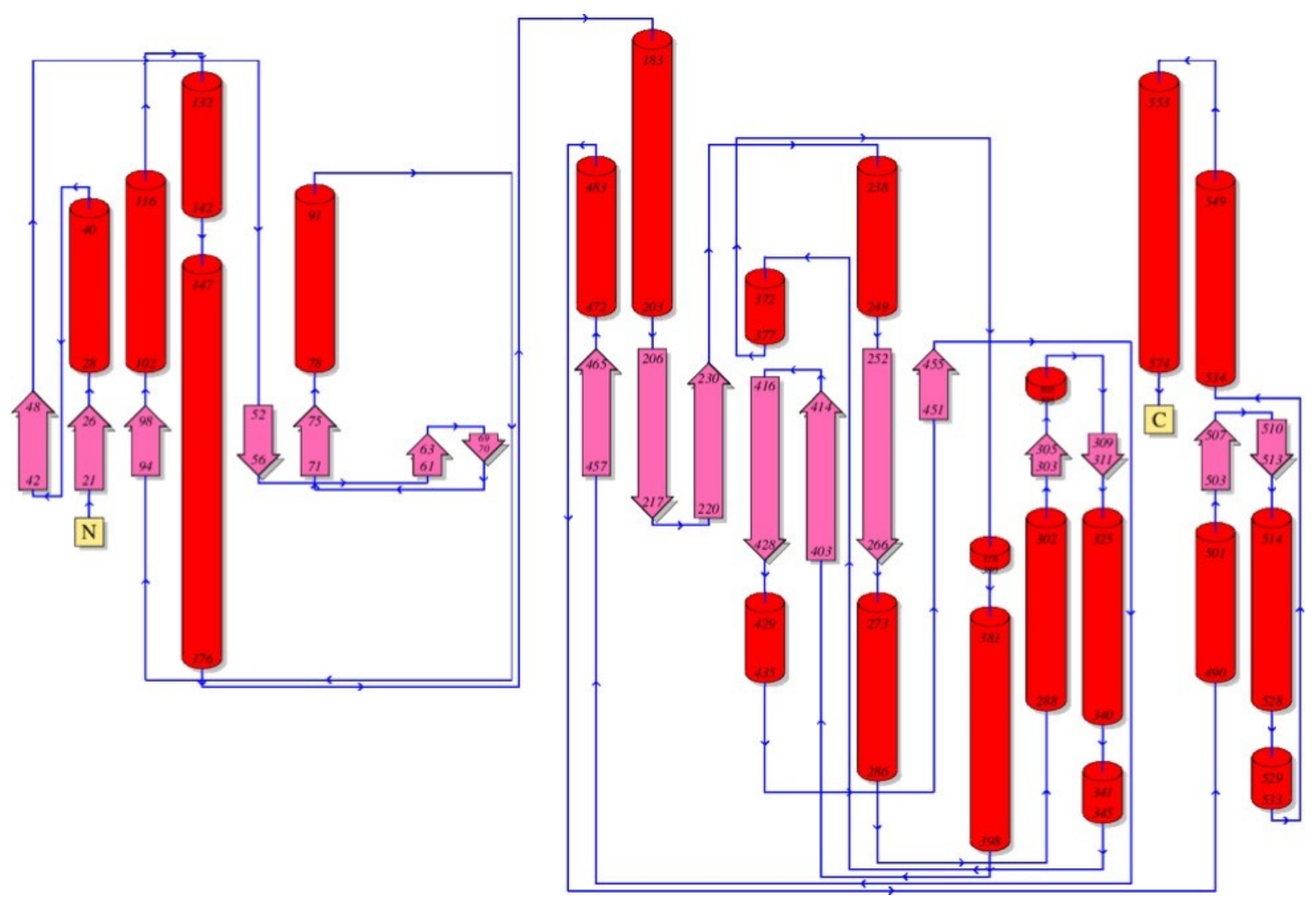

Figure 2-15. SmTopoI_N65 topology diagram.

Topology diagram of SmTopoI_N65 created with PDBsum showing beta strands as pink arrows and alpha helices as red cylinders. ${ }^{121}$

Reprinted from final submission with open access permission, Elsevier Inc. Jones, J. A. \& Hevener, K. E. Crystal structure of the 65-kilodalton amino-terminal fragment of DNA topoisomerase I from the gram-positive model organism Streptococcus mutans. Biochem Biophys Res Commun, https://doi.org/10.1016/j.bbrc.2019.06.034 (2019. In Press). ${ }^{74}$ 


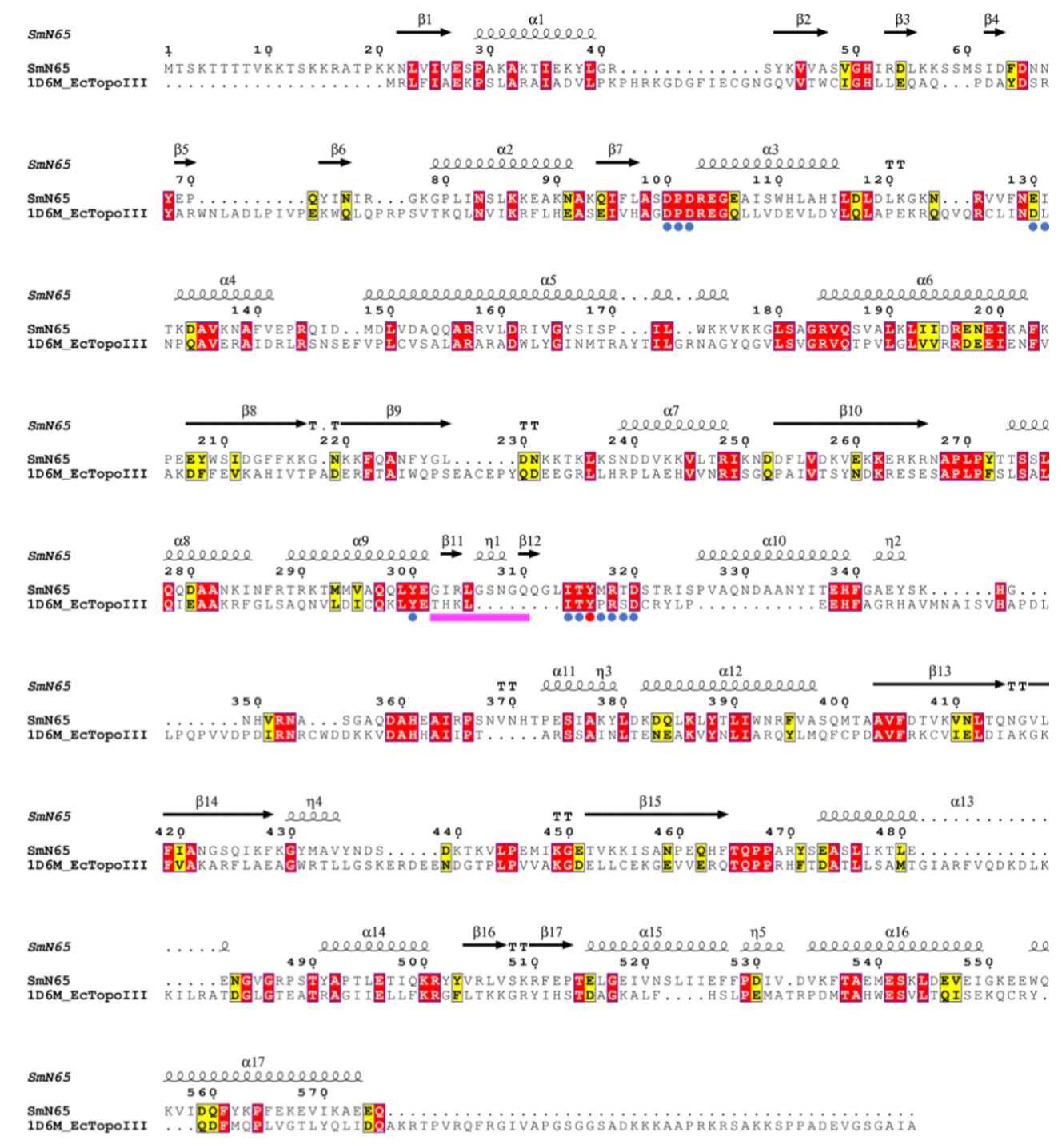

Figure 2-16. Multiple sequence alignment of SmTopoI_N65 and EcTopoIII.

Comparison of SmTopoI_N65, EcTopoIII (PDB 1D6M) via multiple sequence alignment shows moderate sequence identity and similarity, with high sequence identity between the highly conserved active sites. Identical residues are highlighted in red, similar residues are highlighted in yellow, catalytic tyrosine is indicated with a red dot, active site residues within $5 \AA$ from catalytic tyrosine are indicated with blue dots, and the nineresidue loop extension seen in SmTopoI_N65 is indicated with a magenta bar. ${ }^{119,122}$ Reprinted from final submission with open access permission, Elsevier Inc. Jones, J. A. $\&$ Hevener, K. E. Crystal structure of the 65-kilodalton amino-terminal fragment of DNA topoisomerase I from the gram-positive model organism Streptococcus mutans. Biochem Biophys Res Commun, https://doi.org/10.1016/j.bbrc.2019.06.034 (2019. In Press). ${ }^{74}$ 
more rigorous wash steps, and generally resulting in improved removal of non-specific binding contaminant proteins, this concept was also explored by sub-cloning the target gene from the $\mathrm{pET} 15 \mathrm{~b}$ vector into a $\mathrm{pET} 16 \mathrm{~b}$ vector, placing and extended deca-His tag at the N-terminus. ${ }^{134,135}$ Finally, as the small ubiquitin-related modifier (SUMO) fusion system has been reported to increase both target protein solubility and target protein expression levels, the target was cloned into the pET SUMO vector system (SmTopASUMO) in order to analyze the usefulness of an N-term SUMO fusion protein along with an $\mathrm{N}$-term hexa-His tag. ${ }^{136,137}$

Of the various constructs examined, the product of the deca-his-tagged $\mathrm{pET} 16 \mathrm{~b}$ construct (SmTopI16b) resulted in enough of an apparent increase in affinity for the nickel column to be successfully exploited via a step-wise elution to satisfactorily isolate the tagged target protein from the co-eluting contaminant proteins that plagued the hexahis-tagged pET15b construct. Furthermore, enzyme activity was maintained with the deca-his-tagged construct, bypassing the immediate need for cleaving the affinity tag for downstream applications - a luxury not necessarily expected with the larger SUMO fusion tag construct (which also showed promising results in the purification trials).

Because the separation of DNA from DNA-binding proteins like topoisomerase I is essential, yet particularly onerous, a protocol implementing a rapid and efficient means of addressing this obstacle was highly desired. As an alternative to more traditional DNA removal methods, the ionic strength of the buffers used in the IMAC purification step were increased and the $\mathrm{Ni}^{2+}$-NTA-bound target protein was exposed to a high salt wash over an extended period of time in order to successfully disrupt the largely electrostatic nature of DNA-protein binding. ${ }^{138}$ Therefore, in order to further optimize SmTopI16b yield, higher ionic strength buffers were employed to both increase the solubility of the target protein as well as to aid in the expedited removal of DNA during purification steps. Additionally, auto-induction was successfully adopted in order to increase yield and greatly decrease the effort required during the expression and purification protocol. As mini-expression analysis showed no apparent gain or loss in target solubility by adopting auto-induction, it was deduced that the overall increase in target protein yield seen between auto-induction and IPTG-induction likely resulted from greater overall growth.

\section{Unique structural features of SmTopoI_N65}

The SmTopoI_N65 model shows only 3 beta strands in D1, whereas EcTopoI and MtTopoI each contain four D1 beta strands. Within $5 \AA$ from the catalytic center of Y316, only one dissimilar residue is observed between SmTopoI_N65 and EcTopoI_N67, found between the two aspartates on the DXD TOPRIM motif - that is, P101 on SmTopoI_N65, versus L112 on EcTopoI_N67. Interestingly, immediately prior to the conserved active site, SmTopo_N65 displays a unique nine residue loop extension (Figure 2-13 and Figure 2-14) not present in any of the topo I structures previously determined, except for a similar but non-identical extension seen in topo I from the extremophile Thermotoga maritima (PDB 2GAI; Figure 2-17). The SmTopoI_N65 loop extension is mainly polar, with polar uncharged residues S307, N308, Q310, and Q311. 


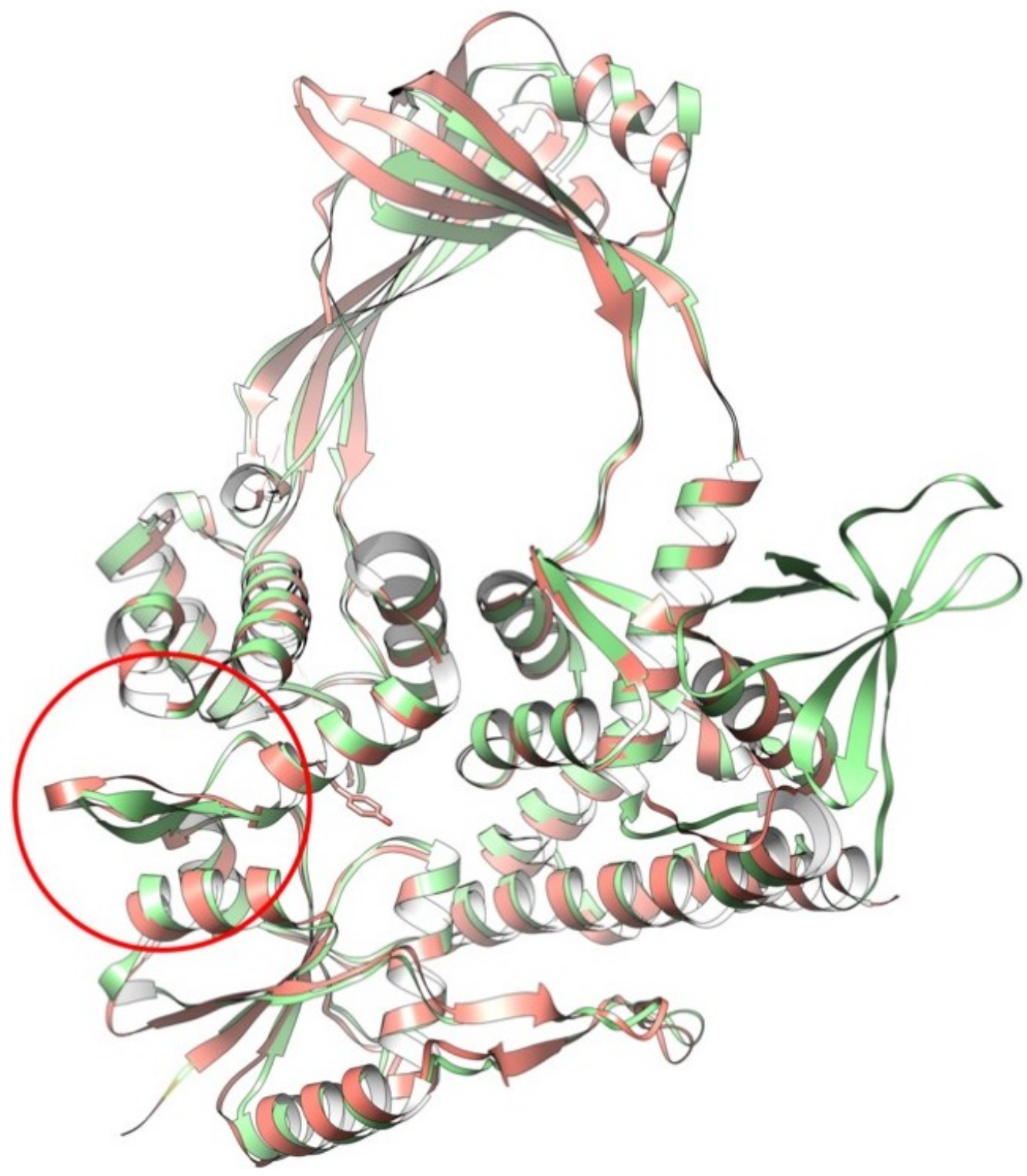

Figure 2-17. Structural alignment of SmTopoI_N65 and TmTopoI (PDB 2GUI)

Structural alignment of SmTopoI_N65 (red) with topo I from the thermophile Thermotoga maritima, TmTopoI (PDB 2GUI; green) shown in flat ribbon format conducted with UCSF Chimera MatchMaker structure comparison tool. ${ }^{95,113}$ The unique loop extension near the active site is highlighted with a red circle and the catalytic Y316 residue on SmTopoI_N65 if shown in stick format.

Reprinted from final submission with open access permission, Elsevier, Inc. ones, J. A. \& Hevener, K. E. Crystal structure of the 65-kilodalton amino-terminal fragment of DNA topoisomerase I from the gram-positive model organism Streptococcus mutans. Biochem Biophys Res Commun, https://doi.org/10.1016/j.bbrc.2019.06.034 (2019. In Press). ${ }^{74}$ 
Further sequence alignment analysis of SmTopoI_N65 against several additional select bacteria shows an intriguing lack of this nine-residue extension in several prominent gut microbes, such as E. coli, Bacteroides ovatus, Bacteroides fragilis, and Bifidobacterium breve, while a similar extension exists not only in the thermophile T. maritima, but also in Staphylococcus aureus, Clostridioides difficile, and Neisseria gonorrhoeae (Figure 2-18). Lastly, as seen in MtTopoI, but dissimilar to EcTopoI_N67, SmTopoI_N65 lacks an equivalent $\alpha 1$ helix in D4.

\section{Summary}

As a DNA binding enzyme, SmTopoI presents inherent difficulties during expression and purification. Nonetheless, an effective, rapid, and simplified protocol was developed for the high-yield expression and purification of bacterial topoisomerase I at over $23 \mathrm{mg} / \mathrm{L}$ estimated at greater than 95\% yield via SDS-PAGE analysis. With such a substantial yield of active and highly pure full-length SmTopoI enzyme, the enzyme can be used for effective biochemical and biophysical techniques that will allow for further target characterization and validation studies, as well as future compound screening campaigns and kinetic studies. Due to a number of circumstances, the full-length enzyme is unfortunately not well-suited for a variety of structural studies. As the enzyme is roughly $83 \mathrm{kDa}$, it is both too small for cryo-electron microscopy and too large for NMR. Additionally, what is presumed to be the highly dynamic nature of the full-length enzyme and the requisite high levels of salt necessary to maintain stability, it is also not wellsuited for X-ray crystallography.

Via rational construction of a shortened construct of SmTopoI, useful structural information has nonetheless been attained from SmTopoI_N65 crystal structure determination. Because of the inherently conserved nature of topo I, SmTopoI_N65 exhibits a substantial degree of sequence identity and similarity with other previously determined topo I structures, including a highly conserved active site and similar overall structure. Therefore, any novel distinction among topo I homologues is likely of significant interest and, as such, the SmTopoI_N65 nine-residue extension located immediately $\mathrm{N}$-term-adjacent to the active site is notably unique as this extension is not seen in topo I structures from either E. coli or M. tuberculosis. What may have gone previously overlooked as an insignificant structural variation in topo I from an extremophile inconsequential to human health (TmTopoI), the presence of this loop extension in S. mutans - a bacterium noted for both its utility in research as a Grampositive model organism and its significance in dental and cardiovascular health - stands as a structural dissimilarity that certainly draws attention and may warrant further analysis. Its proximity to the enzyme active site is indeed intriguing, and important questions regarding any underlying significance, including possible implications related to enzymatic efficiency, target DNA sequence selectivity or stabilization, or even the possibility of rationally designing selective catalytic topo I inhibitors that exploit partial engagement with the extension, remain unanswered. 
Figure 2-18. SmTopoI_N65 multiple sequence alignment with select topo I homologs of undetermined structure.

Comparison of SmTopoI_N65 with homologs from various select bacteria, including E. coli, M. tuberculosis, T. maritima, S. aureus, C. difficile, N. gonorrhoeae, B. ovatus, B. fragilis, and B. breve via multiple sequence alignment. Sequence alignment was generated with the ESPript 3 server (http://espript.ibcp.fr/) using a multiple protein sequence alignment produced with Clustal Omega, with secondary structure information extracted from the SmTopoI_N65 structure. ${ }^{89,93,95,114,115}$ Alignment shows high degree of sequence identity and similarity, including the highly conserved active site. Identical residues are highlighted in red, similar residues are highlighted in yellow, catalytic tyrosine is indicated with a red dot, active site residues within $5 \AA$ from catalytic tyrosine are indicated with blue dots, and the nine residue loop extension seen in SmTopoI_N65 is indicated with a magenta bar. ${ }^{119,122}$

Reprinted from final submission with open access permission, Elsevier, Inc. Jones, J. A. \& Hevener, K. E. Crystal structure of the 65-kilodalton amino-terminal fragment of DNA topoisomerase I from the gram-positive model organism Streptococcus mutans. Biochem Biophys Res Commun, https://doi.org/10.1016/j.bbrc.2019.06.034 (2019. In Press). ${ }^{74}$ 

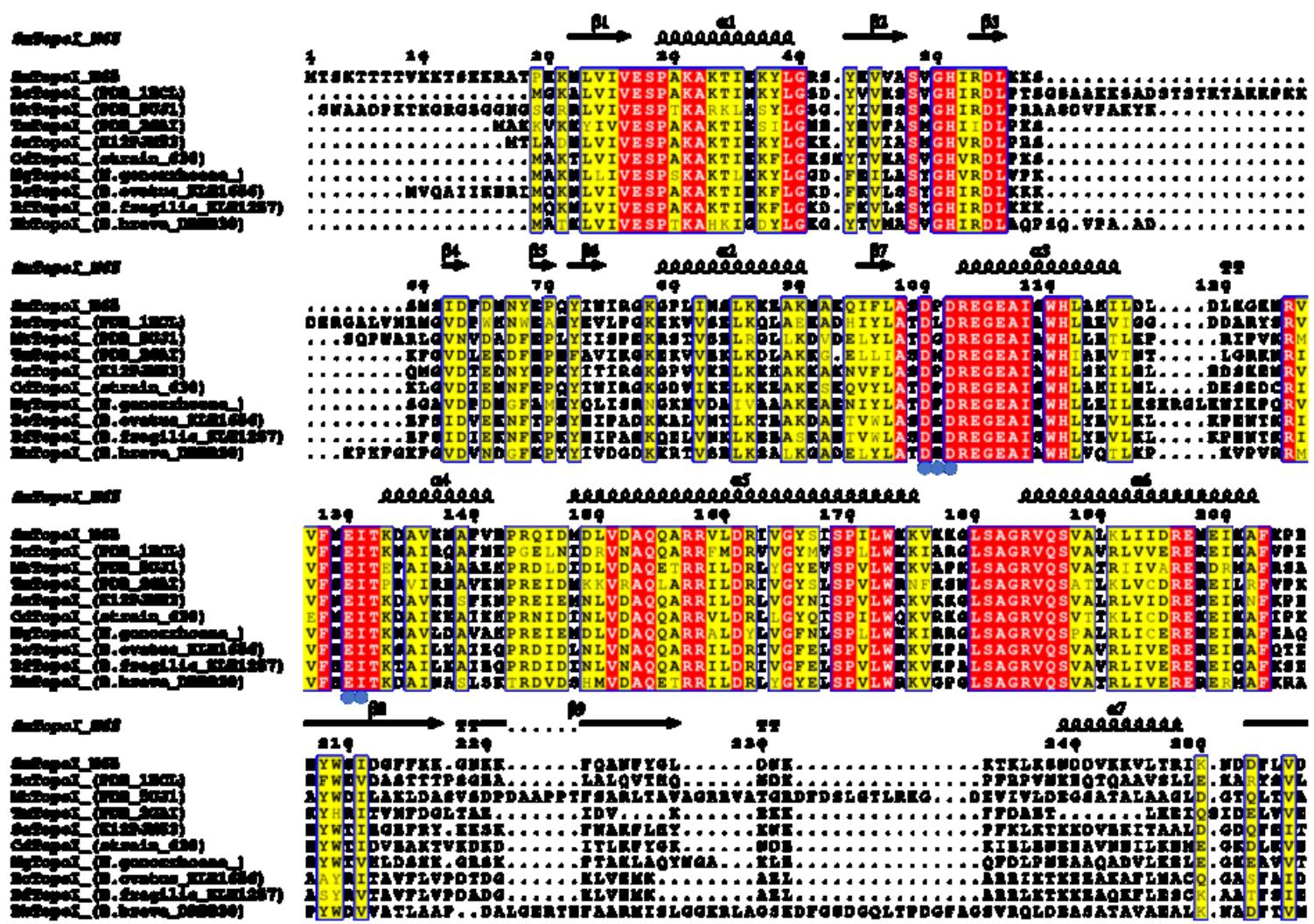

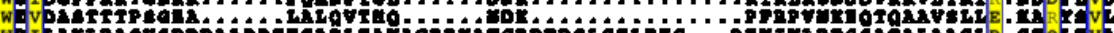

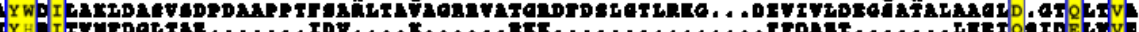

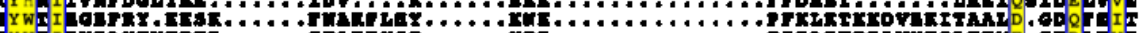

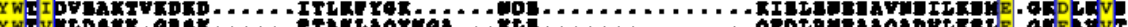
Yw

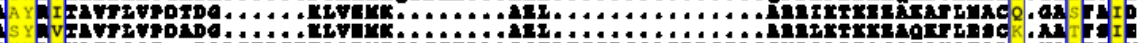

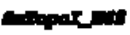
C w

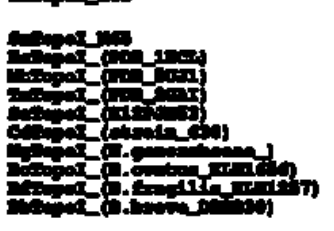

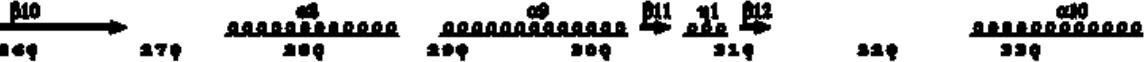

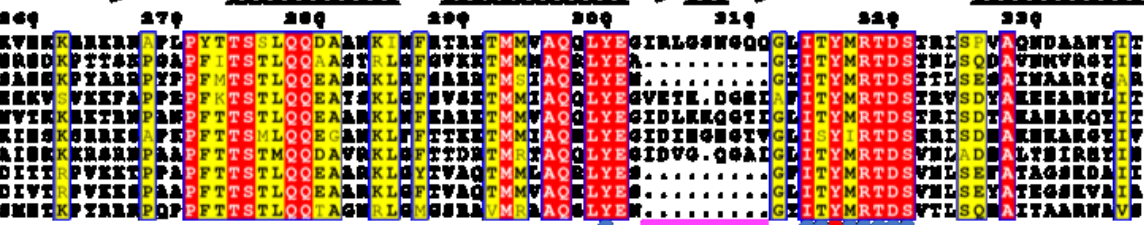
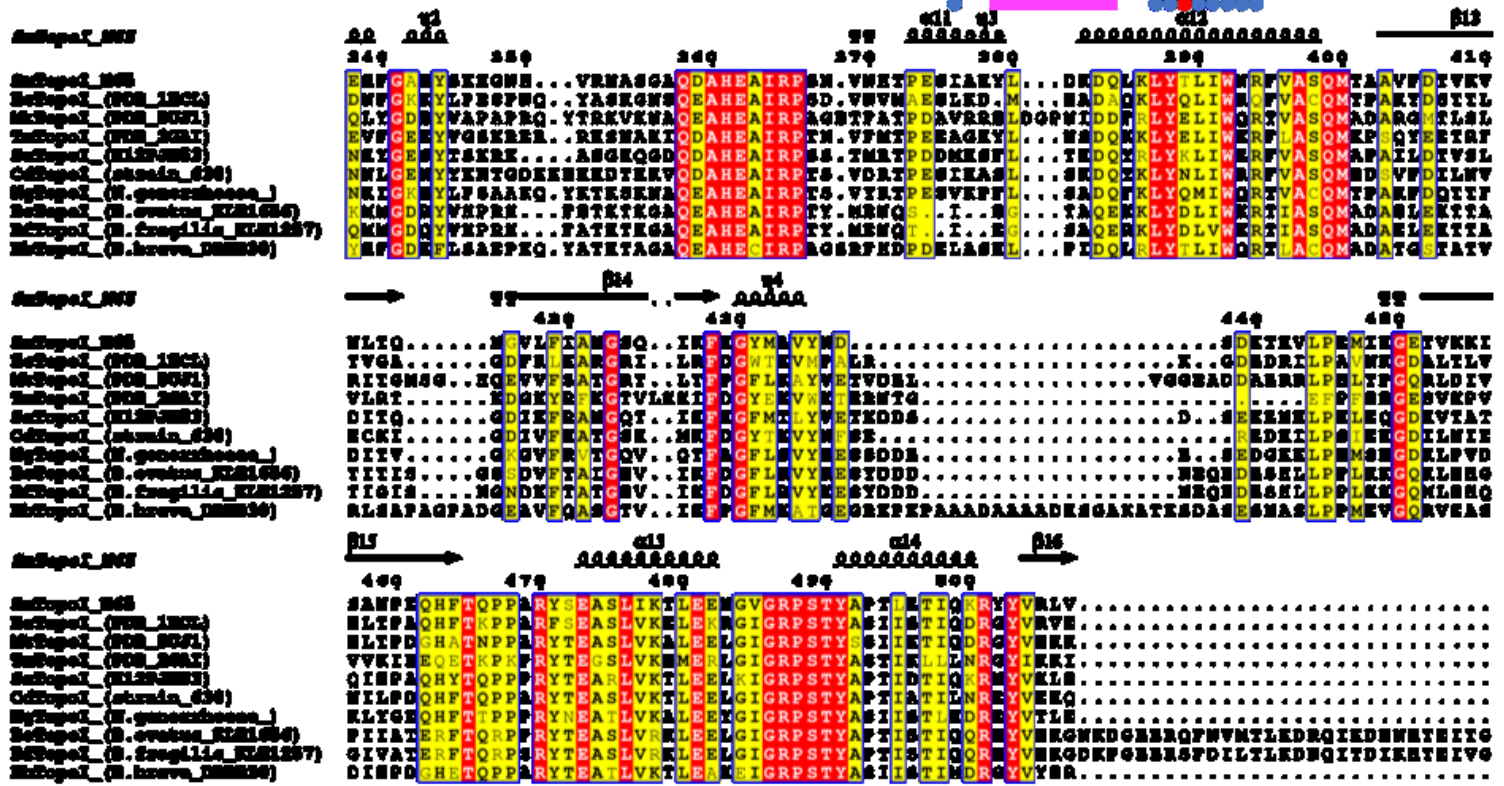

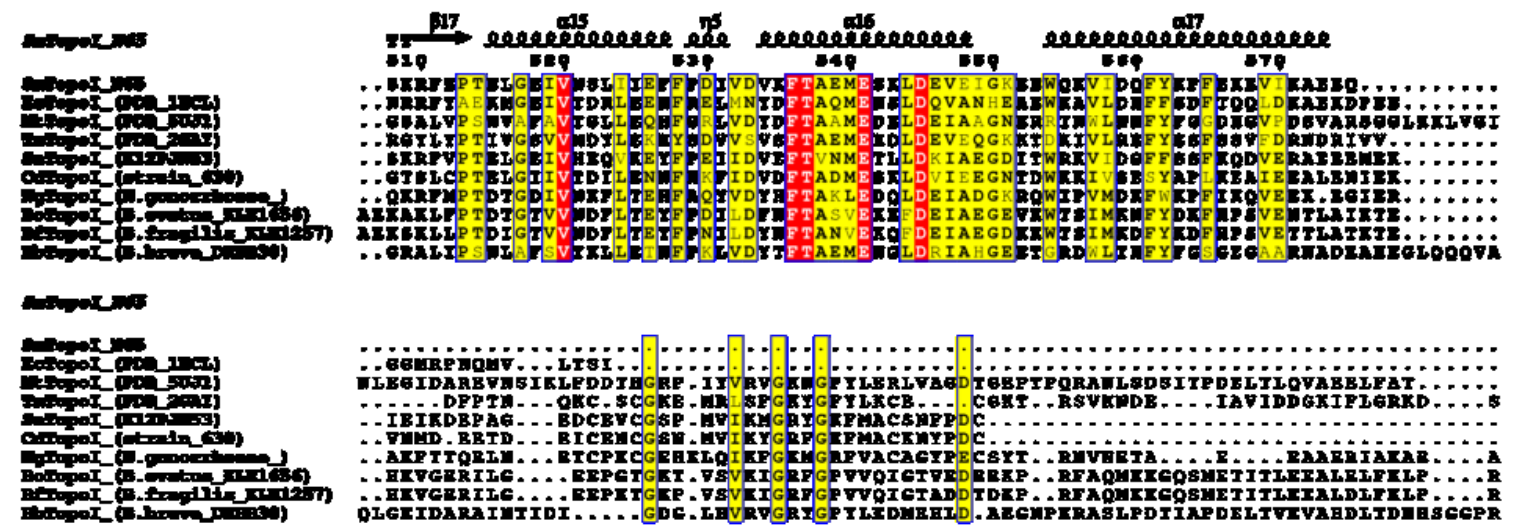

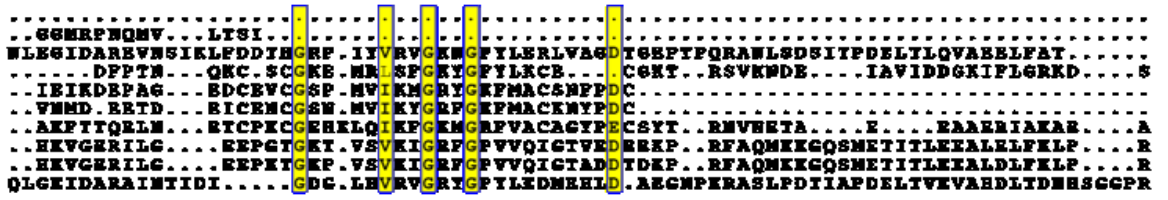
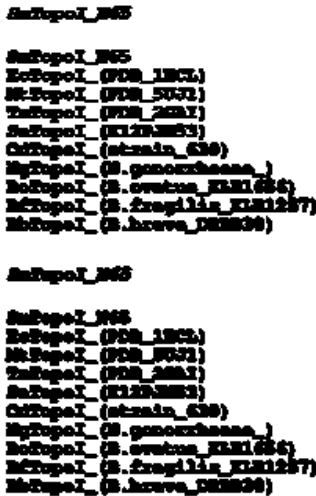

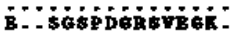

:

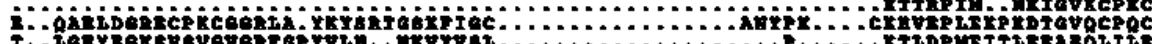

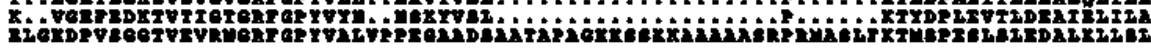

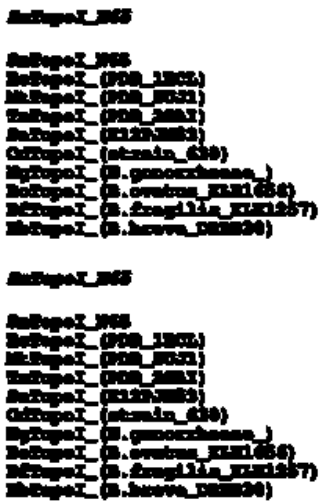

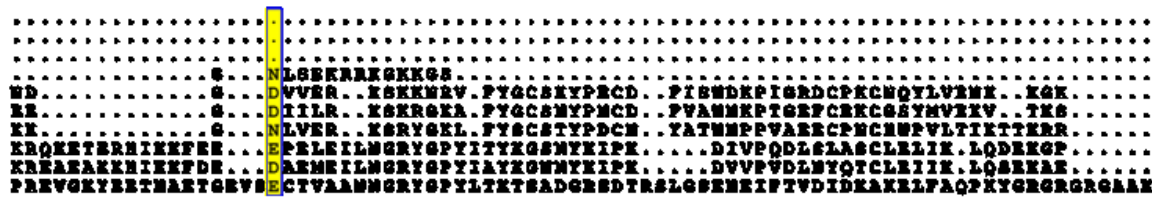

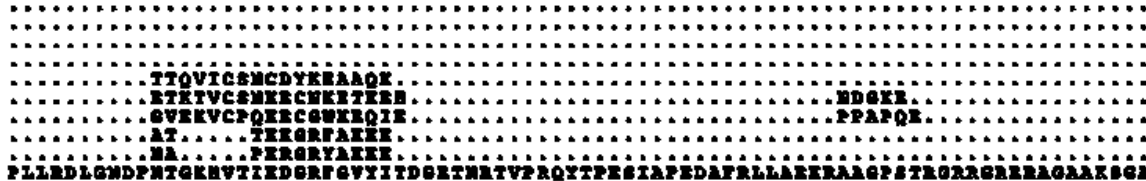

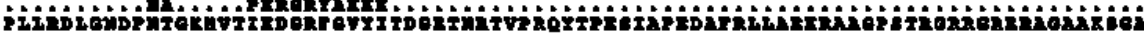

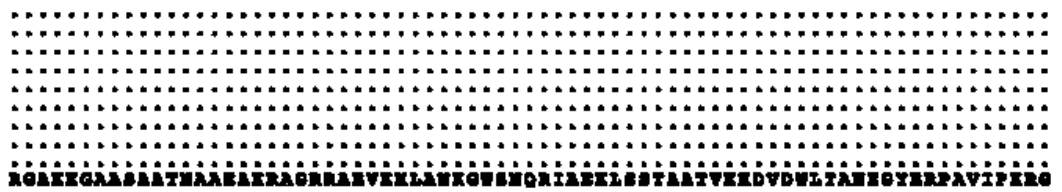

Figure 2-18. Continued. 
Employing X-ray crystallographic methods, this work identified conditions producing crystals that diffracted to $2.06 \AA$ resolution, resulting in the first determined topo I structure from a Gram-positive bacterium, SmTopoI_N65. This topo I fragment structure offers important insight into both the similarities and differences between bacterial topo I from several different relevant organisms. Continued efforts to characterize SmTopoI are still warranted, including but not limited to the structural determination of full-length SmTopoI, co-crystal structures with bound oligonucleotides, enzymatic and kinetic studies, as well as various studies focused on the relatively unique active-site-adjacent nine-residue loop extension highlighted in this work. Such studies regarding the latter topic may include studies deleting, extending, or mutating the loop itself, as well as side-by-side comparison of structural and enzymatic studies of topo I enzymes from various bacterial organisms, including those with and without the loop extension. Nonetheless, the results of these studies have improved our knowledge of topo I and will facilitate future rational research efforts targeting this important enzyme. 


\title{
CHAPTER 3. TARGETING ACYL CARRIER PROTEIN (ACP)-ENOYL REDUCTASE II (FABK): PRODUCTION, STABILIZATION, AND CRYSTALLIZATION ${ }^{3}$
}

\author{
Introduction
}

\section{Background}

Clostridioides difficile (formerly Clostridium difficile) is a gram-positive, sporeforming, anaerobic pathogen that is the leading cause of health care-associated diarrhea and gastroenteritis-associated deaths in the U.S. ${ }^{139-142}$ Clinical consequences of $C$. difficile infection (CDI) range from asymptomatic or mild diarrhea to recalcitrant diarrhea and fatal pseudomembranous colitis. ${ }^{143} \mathrm{CDI}$ is caused by the eradication of key gut flora, such as Bifidobacterium sp. and Bacteroides sp., which normally suppress $C$. difficile overgrowth. ${ }^{144}$ This typically occurs via the use of broad-spectrum antibiotics (e.g., clindamycin, fluoroquinolones, and beta-lactams). ${ }^{144-146}$ The first-line CDI treatment, vancomycin, exhibits activity against beneficial gut flora and results in their eradication, contributing to high rates of recurrence. ${ }^{147,148}$ Further contributing to relapse is the pathogen's ability to produce treatment-resistant spores that survive in the intestinal tract. ${ }^{149,150}$ The emergence of epidemic strains, namely BI/NAP1/027 (North American pulsed-field gel electrophoresis type 1, ribotype 027 ), correlated with an increase in incidence and severity of CDI. ${ }^{147}$ While the alternative first-line drug fidaxomicin is narrow-spectrum and reduces recurrence, it has failed to show evidence of doing so in NAP1-mediated infections. ${ }^{151,152}$ Fecal microbiota transplantation (FMT) shows promise, but long-term safety data is still lacking, including non-infectious microbiota-related metabolic diseases like obesity. ${ }^{153,154}$ While new agents are currently in clinical trials, recent failures highlight the pressing need for novel anti-CDI agents to maintain the discovery pipeline. ${ }^{146,155}$ These issues emphasize that $C$. difficile remains a high-priority candidate for the development of novel narrow-spectrum antibacterial agents.

\section{Bacterial Type II Fatty Acid Biosynthesis (FAS II) Pathway}

During cyclic fatty acid elongation within the bacterial type II fatty-acid synthesis (FAS II) pathway, enoyl-acyl carrier protein (ACP) reductase enzymes catalyze the critical, rate-limiting terminal reduction step. The FAS-II system provides fatty acid precursory components vital to bacterial organisms for various biological processes like membrane phospholipid production and sporulation. ${ }^{156}$ Strong scientific precedent substantiates these enzymes as antimicrobial drug targets, exemplified by isoniazid, a

\footnotetext{
${ }^{3}$ Portions of chapter from final submission adapted with permission from Jones, J. A.* et al. Smallmolecule inhibition of the C. difficile FAS-II enzyme, FabK, results in selective activity. ACS Chem Biol, https://doi.org/10.1021/acschembio.9b00293 (2019. In Press). (*Co-first author.) Copyright (2019) American Chemical Society. ${ }^{139}$
} 
marketed inhibitor of tubercular InhA (enoyl-ACP reductase); triclosan, a well-known antibacterial FabI inhibitor; and the anti-staphylococcal FabI inhibitor afabicin (Debio1450) currently in clinical trials. ${ }^{31,157-159}$ While the existence of four distinct enoyl reductase isozymes (FabI, FabK, FabL, and FabV) in bacteria precludes the development of broad-spectrum antibacterials targeting them, it affords an ideal opportunity for the rational design of narrow-spectrum agents. ${ }^{68-70}$ Bioinformatics analyses of sequenced $C$. difficile genomes indicate it expresses the FabK isozyme as its sole enoyl-ACP reductase. ${ }^{67,160}$

FabK, a member of the triosephosphate isomerase (TIM) barrel family of proteins, is a less abundant isozyme among the four enoyl-ACP reductases, and lacks sequence similarity to FabI, FabL, and FabV, which are members of the short-chain alcohol dehydrogenase/reductase (SDR) superfamily. ${ }^{69,161}$ Like the other isozymes, FabK requires either NADH or NADPH as a cofactor for activity, but unlike its SDR counterparts, FabK is a flavoenzyme that utilizes flavin mononucleotide (FMN) as an active site prosthetic group. FabK is also unique in that it uses a bi-bi double displacement (ping-pong) enzymatic mechanism for reducing its enoyl substrate. ${ }^{69,161-168}$ The SDR enzymes, however, possess a classical Rossman fold for binding their $\mathrm{NAD}(\mathrm{P}) \mathrm{H}$ cofactor and rely on an ordered sequential enzyme mechanism for reducing their enoyl substrates. ${ }^{169,170}$ While there has been recent debate about the essentiality of the FAS-II pathway in Gram-positive bacteria, ${ }^{171-173}$ several groups have shown that possession of the FapR regulatory system over FabT, as seen in S. aureus, prevents full bypass of FAS-II inhibition by exogenous fatty acid uptake. ${ }^{71,72}$ Indeed, it was recently showed that $C$. difficile, which also possesses FapR, behaves in a similar manner as exogenous fatty acids were unable to rescue the pathogen from FAS-II inhibition. ${ }^{67} \mathrm{C}$. difficile also has unique branched fatty acid requirements, further limiting the likelihood of escape from FAS-II inhibition. ${ }^{174,175}$ Recent work also demonstrated the ability of FAS-II inhibition to decrease spore formation by nearly $90 \%$, which in principle could reduce potential for spore survival, associated endogenous recurrence, and spore dissemination. ${ }^{67}$

\section{FabK as a Narrow-spectrum Target}

CDI pharmacotherapy has shown that maximizing therapeutic success includes preventing collateral damage to the GI microbiome. Important gut species such as Gramnegative Bacteroides sp. ${ }^{176}$ express FabI with or without FabK, while Bifidobacterium sp. lack FabK entirely. ${ }^{67}$ Importantly, published FabK inhibitors are inactive against FabI and FabI-bearing strains. ${ }^{164,167}$ Similarly, FabK has demonstrated resistance to FabI inhibitors like triclosan, which are consequently inactive against strains carrying FabK. ${ }^{69,162,177,178}$ Lastly, the ability of bacterial strains carrying two enoyl reductases to survive the deletion of one isozyme suggests that both enzymes would need to be dually inactivated to inhibit the growth of such organisms. ${ }^{68}$ This data justifies the investigation of FabK as a narrow-spectrum target for selective anti-difficile therapy. In an effort to initially characterize the enzyme target, the following research includes $\mathrm{CdFabK}$ 
expression and purification details, preliminary $\mathrm{CdFabK}$ characterization, and continuing $\mathrm{CdFabK}$ protein crystallography work.

\begin{abstract}
Aims
Protein Expression, Purification, and Stabilization of CdFabK

In order to carry out the desired enzyme target characterization and validation, a high yield of high purity $\mathrm{CdFabK}$ was required. Because previous attempts had resulted in low yields of inactive enzyme, a number of different purification methods were tested to maximize the chances of success. As perhaps the most popular initial protein purification is IMAC, this was attempted with various hexa-histidine tag placements, including N-terminal and C-terminal tags. Additionally, native $\mathrm{CdFabK}$ was expressed and purified as tag placement has been reported to impede both enzyme activity and crystallization. ${ }^{179}$

While high quantity and purity of produced target enzyme is indeed of great importance, the desire to develop assays for the screening of inhibitory compounds and to characterize any potential compounds required the need for active and stable compounds. As such, iterative and systematic trials were necessary for determining optimized storage, assay, and crystallization conditions. Herein, the high-yield expression, rapid two-step purification, and stable enzyme activity conditions of FabK from C.difficile is reported. Production methods and can be carried out in a single day with an estimated purity of $95 \%$ or higher.
\end{abstract}

\title{
Crystallization of CdFabK
}

As the three-dimensional structure of CdFabK has not been previously determined, a high resolution structure of $\mathrm{CdFabK}$ is required in order to conduct structural characterization and structure-guided drug discovery efforts. As stated earlier, the CdFabK enzyme is too small for cryo-EM studies and too large for NMR structural studies. Therefore, X-ray crystallography is, by default, necessary for such requisite structural determination and analyses. In order to crystallize CdFabK, a variety of coarse crystallization screens for apo- and co-crystal CdFabK structures were carried out, and subsequent fine screening trials were also conducted. Herein, the conditions for the production of various moderate resolution $\mathrm{CdFabK}$ crystals is reported. 


\section{Materials and Methods}

\section{CdFabK Construct Cloning, Plasmid Production, and Expression}

The fabk gene from $C$. difficile strain 630 was commercially optimized, synthesized, and cloned into the pET15b vector by GenScript (Piscataway, NJ) carrying an N-terminal hexa-histidine tag. ${ }^{139}$ The gene was also cloned into the $\mathrm{pET} 21 \mathrm{~d}$ vector with two different constructs, one carrying a C-term hexa-histidine tag, and the other carrying no tag and encoding only the native enzyme as the gene of interest. The respective plasmid products $(\mathrm{CdFabK}$ 15b, CdFabK_21d, and CdFabK_21d_NATIVE, respectively) were then transformed into XL1-Blue Supercompetent cells (Agilent Technologies, Santa Clara, CA) per protocol. Upon successful transformation, the plasmids were then isolated with the QIAprep Spin Miniprep Kit via protocol and the sequences were confirmed via the University of Tennessee Health Science Center (UTHSC) Molecular Research Center (MRC) sequencing services (Memphis, TN). After sequence confirmation, the plasmids were also transformed into BL21(DE3)-Gold Competent cells (Agilent Technologies, Santa Clara, CA) via protocol.

The proteins were overexpressed in E. coli BL21(DE3)-Gold Competent cells (Agilent Technologies, Santa Clara, CA) cultivated at $37^{\circ} \mathrm{C}$ in TB medium with 100 $\mu \mathrm{g} / \mathrm{mL}$ ampicillin by transferring $1 \%(\mathrm{v} / \mathrm{v})$ overnight culture into fresh medium. Cells were allowed to grow until they reached an $\mathrm{OD}_{600}$ of $\sim 0.6$ and the protein expression was induced with $0.1 \mathrm{mM}$ IPTG supplemented with $0.5 \mathrm{mM}$ flavin mononucleotide (FMN). Growth temperature was decreased to $18^{\circ} \mathrm{C}$ and cells were allowed to grow further for 18 hours at $18^{\circ} \mathrm{C}$. After induction and growth, the cells were harvested by centrifugation at $18000 \mathrm{x} g$ for 15 minutes. Pellets were stored at $-80^{\circ} \mathrm{C}$ until use.

\section{CdFabK_15b and CdFabK_21d Purification}

Pellets were resuspended in CFabK Buffer A (20 mM HEPES buffer $\mathrm{pH} 8.0$ with $300 \mathrm{mM} \mathrm{NH}_{4} \mathrm{Cl}, 1 \mathrm{mM}$ DTT, $18 \%$ glycerol, $10 \mu \mathrm{g} / \mathrm{mL}$ benzamidine, and $10 \mathrm{mM}$ imidazole). Lysis components were then added, including $0.5 \mathrm{mg} / \mathrm{mL}$ lysozyme, 10 $\mu \mathrm{g} / \mathrm{mL}$ DNaseI, $5 \mathrm{mM} \mathrm{MgCl} 2,0.5 \%$ Triton-X 100, $25 \mathrm{mM}$ sucrose, and 1 Pierce ${ }^{\circledR}$ EDTA-free Protease inhibitor tablet per $50 \mathrm{~mL}$. Cells were lysed at $4{ }^{\circ} \mathrm{C}$ with gentle stirring, then sonicated at $50 \%$ amplitude on ice for eight minutes with eight seconds on and 24 seconds off. Lysates were centrifuged at 39,000 $\times$ g for 15 minutes and the supernatants were filtered through a 0.22 polyethersulfone (PES) syringe filter (EMD Millipore, Billerica, MA).

His-tagged proteins were purified via immobilized metal affinity chromatography (IMAC) by loading the filtered lysate on a HisTrap HP column (GE Life Sciences) in CdFabK Buffer A, washed over 20 column volumes with CdFabK Buffer A, and eluted via linear gradient to $100 \%$ CdFabK Buffer B (CdFabK Buffer A with $500 \mathrm{mM}$ imidazole) over 20 column volumes. Peak eluted proteins were pooled and further 
purified by SEC on a HiLoad 26/600 Superdex 200 PG size exclusion column (GE Healthcare Life Sciences, Pittsburgh, PA) in CdFabK SEC Buffer (50 mM HEPES pH $8.0,300 \mathrm{mM} \mathrm{NH}_{4} \mathrm{Cl}, 18 \%$ glycerol, and $3 \mathrm{mM}$ DTT) via isocratic elution. After purification, proteins were concentrated via 10,000 MWCO Amicon ${ }^{\mathrm{TM}}$ Ultra-15 Centrifugal Filter Units and brought to $100 \mu \mathrm{M}$ with $38.5 \%$ glycerol, snap frozen in liquid nitrogen, and stored at $-80^{\circ} \mathrm{C}$ until future use. Proteins have been confirmed active up to six months after storage under these conditions. Enzyme was confirmed via SDSPAGE gel analysis (Mini-PROTEAN ${ }^{\circledR}$ TGX Stain-Free ${ }^{\text {TM }}$ Pre-Cast Gel and Precision Plus Protein ${ }^{\mathrm{TM}}$ Unstained Standard, Bio-Rad Laboratories, Hercules, CA) and the enzyme activity was analyzed in an Implen NP80 nanophotometer as described below (West Lake Village, CA). Enzyme concentration was determined via Pierce ${ }^{\mathrm{TM}}$ 660nm Protein Assay Reagent (ThermoFisher Scientific, Waltham, MA) per protocol.

\section{CdFabK_21d_NATIVE Purification}

Pellets were resuspended in Anion Buffer A (20 mM BIS TRIS Propane buffer pH 9.5, $1 \mathrm{mM} \mathrm{DTT}$, and $18 \%$ glycerol). Lysis components were then added, including $0.5 \mathrm{mg} / \mathrm{mL}$ lysozyme, $10 \mu \mathrm{g} / \mathrm{mL}$ DNaseI, $0.5 \%$ Triton-X 100, and 1 Pierce ${ }^{\circledR}$ EDTA-free Protease inhibitor tablet per $50 \mathrm{~mL}$. Cells were lysed at $4{ }^{\circ} \mathrm{C}$ with gentle stirring, then sonicated at 50\% amplitude for eight minutes with eight seconds on and 24 seconds off. Lysates were centrifuged at $39,000 \times \mathrm{g}$ for 15 minutes and the supernatants were filtered through a 0.22 PES syringe filter (EMD Millipore, Billerica, MA).

Native CdFabK protein (CdFabK_NATIVE) was purified via anion exchange chromatography by loading the filtered lysate on HiTrap Q HP GE Column (GE Life Sciences) in Anion Buffer A, subsequently washed with 20 column volumes with Anion Buffer A, and then eluted via linear gradient to $100 \%$ Anion Buffer B (Anion Buffer A with $500 \mathrm{mM} \mathrm{NH}_{4} \mathrm{Cl}$ ) over 20 column volumes. The $450 \mathrm{~nm}$ wavelength was followed to discern fractions containing FabK cofactor, flavin mononucleotide (FMN), which was followed to track the presence of the CdFabK_NATIVE protein. Fractions were collected and pooled, enzyme was confirmed via PAGE gel analysis, and the enzyme activity was analyzed in an Implen NP80 nanophotometer as described below (West Lake Village, CA). Enzyme concentration was determined via Pierce ${ }^{\mathrm{TM}}$ 660nm Protein Assay Reagent (ThermoFisher Scientific (Waltham, MA, USA) per protocol.

\section{CdFabK Activity and Stability Assays}

\section{Salt trials}

All activity and stability assays were carried out at $25^{\circ} \mathrm{C}$. Trials were conducted in the presence of $50 \mathrm{nM} \mathrm{CdFabK}$ _15b N-term hexa-His tagged target enzyme, 5\% DMSO, $150 \mu \mathrm{M}$ Cro-CoA, and $150 \mu \mathrm{M}$ NADH in an overall volume of $100 \mu \mathrm{L}$ in the following various assay buffers: CdFabK Salt Assay Buffer 1 (HEPES pH 7.5, $0 \mathrm{mM} \mathrm{NH}_{4} \mathrm{Cl}$, and 4\% glycerol), CdFabK Salt Assay Buffer 2 (HEPES pH 7.5, $100 \mathrm{mM} \mathrm{NH}_{4} \mathrm{Cl}$, and 4\% 
glycerol), CdFabK Salt Assay Buffer 3 (HEPES pH 7.5, $300 \mathrm{mM} \mathrm{NH}_{4} \mathrm{Cl}$, and 4\% glycerol), and CdFabK Salt Assay Buffer 4 (HEPES pH 7.5, $500 \mathrm{mM} \mathrm{NH}_{4} \mathrm{Cl}$, and 4\% glycerol). DMSO and enzyme were incubated together for 5 minutes before the Cro-CoA substrate was added and the reaction was started immediately afterward via the addition of NADH. NADH absorbance ( $340 \mathrm{~nm}$ ) was measured in a Biotek Synergy H1 microplate reader (Winooski, VT) in a final volume of $100 \mu \mathrm{L}$ in Greiner Bio-One ${ }^{\mathrm{TM}}$ 384-Well $\mu$ Clear $^{\mathrm{TM}}$ Bottom Polystyrene Microplates (Monroe, NC) via 20 second intervals for 7 minutes to evaluate the rate of reaction.

\section{Glycerol trials}

Trials were conducted in the presence of $50 \mathrm{nM} \mathrm{CdFabK} \_15 \mathrm{~b}$ N-term hexa-His tagged target enzyme, 5\% DMSO, $150 \mu \mathrm{M}$ Cro-CoA, and $150 \mu \mathrm{M}$ NADH in an overall volume of $100 \mu \mathrm{L}$ in the following various assay buffers: CdFabK Glycerol Assay Buffer 1 (HEPES pH 7.5, $500 \mathrm{mM} \mathrm{NH}_{4} \mathrm{Cl}$, and 0\% glycerol), CdFabK Glycerol Assay Buffer 2 (HEPES pH 7.5, $500 \mathrm{mM} \mathrm{NH}_{4} \mathrm{Cl}$, and 4\% glycerol), CdFabK Glycerol Assay Buffer 3 (HEPES pH 7.5, $500 \mathrm{mM} \mathrm{NH}_{4} \mathrm{Cl}$, and 10\% glycerol), and CdFabK Glycerol Assay Buffer 4 (HEPES pH 7.5, $500 \mathrm{mM} \mathrm{NH}_{4} \mathrm{Cl}$, and $15 \%$ glycerol). DMSO and enzyme were incubated together for 5 minutes before the Cro-CoA substrate was added and the reaction was started immediately afterward via the addition of NADH. NADH absorbance $(340 \mathrm{~nm})$ was measured in a Biotek Synergy H1 microplate reader (Winooski, VT) in a final volume of $100 \mu \mathrm{L}$ in Greiner Bio-One ${ }^{\mathrm{TM}} 384-\mathrm{Well} \mu \mathrm{Clear}^{\mathrm{TM}}$ Bottom Polystyrene Microplates (Monroe, NC) via 20 second intervals for 7 minutes to evaluate the rate of reaction.

\section{Buffer and pH trials}

Trials were conducted in the presence of $50 \mathrm{nM} \mathrm{CdFabK} 15 \mathrm{~b}$ N-term hexa-His tagged target enzyme, 5\% DMSO, $150 \mu \mathrm{M}$ Cro-CoA, and $150 \mu \mathrm{M}$ NADH in an overall volume of $100 \mu \mathrm{L}$ in the following various assay buffers: CdFabK pH Assay Buffer 1 (100 mM MES pH 5.5, $500 \mathrm{mM} \mathrm{NH}_{4} \mathrm{Cl}$, and 10\% glycerol), CdFabK pH Assay Buffer 2 (100 mM MES pH 6.5, $500 \mathrm{mM} \mathrm{NH}_{4} \mathrm{Cl}$, and 10\% glycerol), CdFabK pH Assay Buffer 3 (100 mM HEPES pH 7.5, $500 \mathrm{mM} \mathrm{NH}_{4} \mathrm{Cl}$, and 10\% glycerol), CdFabK pH Assay Buffer 4 (100 mM HEPES pH 8.5, $500 \mathrm{mM} \mathrm{NH}_{4} \mathrm{Cl}$, and 10\% glycerol), and CdFabK pH Assay Buffer 5 (100 mM CHES pH 9.5, $500 \mathrm{mM} \mathrm{NH}_{4} \mathrm{Cl}$, and 10\% glycerol). DMSO and enzyme were incubated together for 5 minutes before the Cro-CoA substrate was added and the reaction was started immediately afterward via the addition of NADH. NADH absorbance $(340 \mathrm{~nm})$ was measured in a Biotek Synergy H1 microplate reader (Winooski, VT) in a final volume of $100 \mu \mathrm{L}$ in Greiner Bio-One ${ }^{\mathrm{TM}}$ 384-Well $\mu \mathrm{Clear}^{\mathrm{TM}}$ Bottom Polystyrene Microplates (Monroe, NC) via 20 second intervals for 7 minutes to evaluate the rate of reaction. 


\section{CdFabK Stability Trials}

\section{Initial $\gamma$-Globulins dilution assays}

Stability assays were carried out at $25^{\circ} \mathrm{C}$ in the presence of $50 \mathrm{nM} \mathrm{CdFabK} \_15 \mathrm{~b}$ $\mathrm{N}$-term hexa-His tagged target enzyme, 10\% DMSO, $150 \mu \mathrm{M}$ Cro-CoA, and $150 \mu \mathrm{M}$ $\mathrm{NADH}$ in an overall volume of $100 \mu \mathrm{L}$, in CdFabK Assay Buffer (HEPES pH 8.0, 500 $\mathrm{mM} \mathrm{NH}_{4} \mathrm{Cl}$, and $10 \%$ glycerol). The enzyme was diluted from the $100 \mu \mathrm{M}$ storage stock concentration to $1 \mu \mathrm{M}$ working stock concentration in CdFabK Assay Buffer with varying amounts of $\gamma$-Globulins (Sigma Aldrich, St. Louis, MO)—ranging from $0 \mathrm{mg} / \mathrm{mL}$ $(0 \mathrm{mg} / \mathrm{mL}$ overall assay concentration) to $2.5 \mathrm{mg} / \mathrm{mL}(0.125 \mathrm{mg} / \mathrm{mL}$ overall assay concentration), and then allowed to incubate at $25^{\circ} \mathrm{C}$ for 0,30 , and 60 minutes. Enzyme from each time point and $\gamma$-Globulin dilution concentration was then incubated with DMSO as a positive control for 10 minutes before the Cro-CoA substrate was added and the reaction was started immediately afterward via the addition of $50 \mu \mathrm{L} 300 \mu \mathrm{M}$ NADH. NADH fluorescence $(340 \mathrm{~nm} / 460 \mathrm{~nm})$ was measured in a Biotek Synergy H1 microplate reader (Winooski, VT) in a final volume of $100 \mu \mathrm{L}$ in Greiner Bio-One ${ }^{\mathrm{TM}} 384-$ Well $\mu$ Clear $^{\mathrm{TM}}$ Bottom Polystyrene Microplates (Monroe, NC) via 20 second intervals for 10 minutes to evaluate the rate of reaction.

\section{Optimizing $\gamma$-Globulins dilution assays}

Maximal CdFabK dilution assays were carried out at $25^{\circ} \mathrm{C}$ in the presence of twofold dilutions of $50 \mathrm{nM} \mathrm{CdFabK}$ 15b N-term hexa-His tagged target enzyme down to $6.25 \mathrm{nM} \mathrm{CdFabK} \_15 \mathrm{~b}$. Assays were carried out in 10\% DMSO, $300 \mu \mathrm{M}$ Cro-CoA, and $125 \mu \mathrm{M}$ NADH in an overall volume of $100 \mu \mathrm{L}$, in CdFabK Assay Buffer (HEPES pH 8.0, $500 \mathrm{mM} \mathrm{NH}_{4} \mathrm{Cl}$, and $10 \%$ glycerol). The enzyme was diluted from the $100 \mu \mathrm{M}$ storage stock concentration to $1 \mu \mathrm{M}, 0.5 \mu \mathrm{M}, 0.25 \mu \mathrm{M}$, and $0.125 \mu \mathrm{M}$ working stock concentrations in CdFabK Assay Buffer with an increased $5 \mathrm{mg} / \mathrm{mL} \quad \gamma$-Globulins $(0.25$ $\mathrm{mg} / \mathrm{mL}$ overall assay concentration), and then allowed to incubate at $25^{\circ} \mathrm{C}$ for 30 minutes. Enzyme was then incubated in assay buffer with DMSO as a positive control for 10 minutes before the Cro-CoA substrate was added and the reaction was started immediately afterward via the addition of $50 \mu \mathrm{L} 250 \mu \mathrm{M}$ NADH. NADH fluorescence $(340 \mathrm{~nm} / 460 \mathrm{~nm}$ ) was measured in a Biotek Synergy H1 microplate reader (Winooski, VT) in a final volume of $100 \mu \mathrm{L}$ in Greiner Bio-One ${ }^{\mathrm{TM}}$ 384-Well $\mu$ Clear ${ }^{\mathrm{TM}}$ Bottom Polystyrene Microplates (Monroe, NC) via 15 second intervals for 30 minutes to evaluate the rate of reaction and to monitor for any eventual loss of enzyme activity.

\section{CdFabK Crystallization}

\section{CdFabK_21d crystals}

C-term hexa-histidine tagged CdFabK_21d was crystallized at $5 \mathrm{mg} \mathrm{mL}^{-1}$ in a $3 \mathrm{uL}: 3 \mathrm{uL}$ 1:1 ratio protein to condition using the hanging-drop vapor-diffusion method off 
of a coarse-matrix screen. The initial crystal growth condition was Morpheus (Molecular Dimensions, Maumee, $\mathrm{OH})$ condition 1-48 (0.12 M alcohols mix of 0.2 M 1,6hexanediol, 0.2 M 1-butanediol, 0.2 M 1,2-propanediol, 0.2 M 2-propanol, 0.2 M 1,4butanediol, and 0.2 M 1,3-propanediol; $0.1 \mathrm{M}$ buffer mix of Tris base and BICINE, $\mathrm{pH}$ 8.5; and $37.5 \% \mathrm{v} / \mathrm{v}$ precipitant mix of $25 \% \mathrm{v} / \mathrm{v} 2$-methyl-2,4-pentanediol, $25 \%$ polyethylene glycol 1000 , and $25 \%$ polyethylene glycol 3500 ) at $18^{\circ} \mathrm{C}$. Crystals were collected for X-ray diffraction analysis. Crystals were grown in 48-well VDX plates with sealant (Hampton Research, Aliso Viejo, CA) and were visible within one week.

\section{CdFabK_15b crystals}

$\mathrm{N}$-term hexa-histidine tagged CdFabK $15 \mathrm{~b}$ was crystallized at $4.8 \mathrm{mg} \mathrm{mL}^{-1}$ in a $7 \mathrm{uL}: 7 \mathrm{uL} \mathrm{1:1} \mathrm{ratio} \mathrm{protein} \mathrm{to} \mathrm{condition} \mathrm{using} \mathrm{the} \mathrm{hanging-drop} \mathrm{vapor-diffusion} \mathrm{method} \mathrm{off}$ of a coarse-matrix screen. The initial crystal growth condition was Morpheus (Molecular Dimensions, Maumee, $\mathrm{OH})$ condition 2-48 (0.1 M amino acids mix of 0.2 M DLglutamic acid monohydrate, 0.2 M DL-alanine, 0.2 M glycine, 0.2 M DL-lysine monohydrochloride, and 0.2 M DL-serine; $0.1 \mathrm{M}$ buffer mix of Tris base and BICINE, $\mathrm{pH} 8.5$; and $37.5 \% \mathrm{v} / \mathrm{v}$ precipitant mix of $25 \% \mathrm{v} / \mathrm{v} 2$-methyl-2,4-pentanediol, $25 \%$ polyethylene glycol 1000 , and $25 \%$ polyethylene glycol 3500 ) at $18^{\circ} \mathrm{C}$. Crystals were collected for X-ray diffraction analysis. Optimized crystals were then obtained via cocrystallization in the same Morpheus $2-48$ condition at $4.8 \mathrm{mg} \mathrm{ml}^{-1}$ in $7 \mathrm{uL}: 7 \mathrm{uL} \mathrm{1:1} \mathrm{ratio}$ of protein to a mix of $93 \%$ crystallization condition and $7 \% 10 \mathrm{mM}$ phenylimidazole compound 1g (see Chapter 4) in DMSO. Crystals were obtained using the hanging-drop vapor-diffusion method. Crystals were grown in 48-well VDX plates with sealant (Hampton Research, Aliso Viejo, CA) and were visible within five days.

\section{Results}

\section{Cloning and Expression of Recombinant CdFabK}

Substantial amounts of pure, active target $\mathrm{CdFabK}$ protein are needed for the various characterization and validation studies required for effective CdFabK-directed drug discovery and development efforts. Again, cloning into various histidine affinity tag systems was chosen and implemented. For the sake of speed, the $C$. difficile fabK gene was commercially optimized, cloned, and inserted into the $\mathrm{pET} 15 \mathrm{~b}$ vector (CdFabK_15b), introducing the popular hexa-Histidine (hexa-His) tag at the N-terminus of the target enzyme for effective first-round purification. ${ }^{123}$ For thorough analysis, the target gene was previously cloned into several alternative vectors to study the effects of affinity tag placement at either the amino or carboxy terminus, as well as native enzyme without any tag. BL21(DE3)-Gold Competent cells were used for the over-expression of target protein. Yellow pellets were noticed upon growth and expression of all three constructs. 


\section{Purification of Recombinant CdFabK Constructs}

As a traditional first step purification method for many target enzymes, $\mathrm{Ni}^{2+}$-NTA IMAC purification of CdFabK with the pET21d C-term hexa-His tag system and the pET15b N-term hexa-His tag system both resulted in high yields (greater than $25 \mathrm{mg} / \mathrm{L}$ ) of initially active enzyme when high levels of glycerol, such as $18 \%$, were used throughout the purification steps. As the C-term hexa-His tagged enzyme (CdFabK_21d) proved to lose activity rapidly, the N-term hexa-His tagged construct (CdFabK_15b) became the construct of choice. Following successful IMAC purification, further purification of CdFabK_15b was carried out via size exclusion chromatography and resulted in highly pure enzyme of at least $95 \%$ purity via SDS-PAGE analysis (Figure 3-1) and high overall yields greater than $10 \mathrm{mg} / \mathrm{L}$ determined via $660 \mathrm{~nm}$ protein concentration assay with BSA as a standard. Target enzyme solubility was determined satisfactory for immediate pooling and purifying via SEC without needing to buffer exchange or dialyze, leading to a highly rapid and simple purification protocol that can be conducted in one to two days. Under current storage conditions at $-80^{\circ} \mathrm{C}$ (Materials and Methods), enzyme has been determined active for over six months.

The native CdFabK target enzyme was also purified via preliminary anion exchange chromatography, but the purity in comparison to the $\mathrm{N}$-term hexa-His tagged construct was quite poor and further protocol development for purifying the native target enzyme was suspended for the time being.

\section{Characterization of CdFabK}

As the overall stability of CdFabK_21d had been determined previously to be very poor, CdFabK_15b was expressed, purified, and tested for comparative stability. Via systematic stability and activity trials, effective assay conditions were determined for $\mathrm{CdFabK}$ _ $15 \mathrm{~b}$ with a trend of relatively high $\mathrm{NH} 4 \mathrm{Cl}$, relatively high glycerol, and slightly basic $\mathrm{pH}$ conditions combining for optimal conditions of HEPES pH 8.0, $500 \mathrm{mM}$ $\mathrm{NH}_{4} \mathrm{Cl}$, and 10\% glycerol (Figure 3-2). Even with the optimized assay conditions, however, the target enzyme lost activity soon after being diluted to concentrations needed for making a working enzyme stock solution for compound screening. The addition of just $2.5 \mathrm{mg} / \mathrm{mL} \gamma$-Globulins to the dilution buffer was determined to greatly increase the duration of activity of the diluted enzyme at an overall concentration of $50 \mathrm{nM}$ from just a few minutes up to at least one hour (Figure 3-3). Additionally, increasing the $\gamma$ Globulins even further to $5 \mathrm{mg} / \mathrm{mL}$ and doubling the substrate concentration to account for extended assay duration to $300 \mu \mathrm{M}$ allowed for further dilutions of enzyme concentration down to single-digit nanomolar $(6.25 \mathrm{nM})$ with the capability of sustained activity for at least 30 minutes (Figure 3-4). 


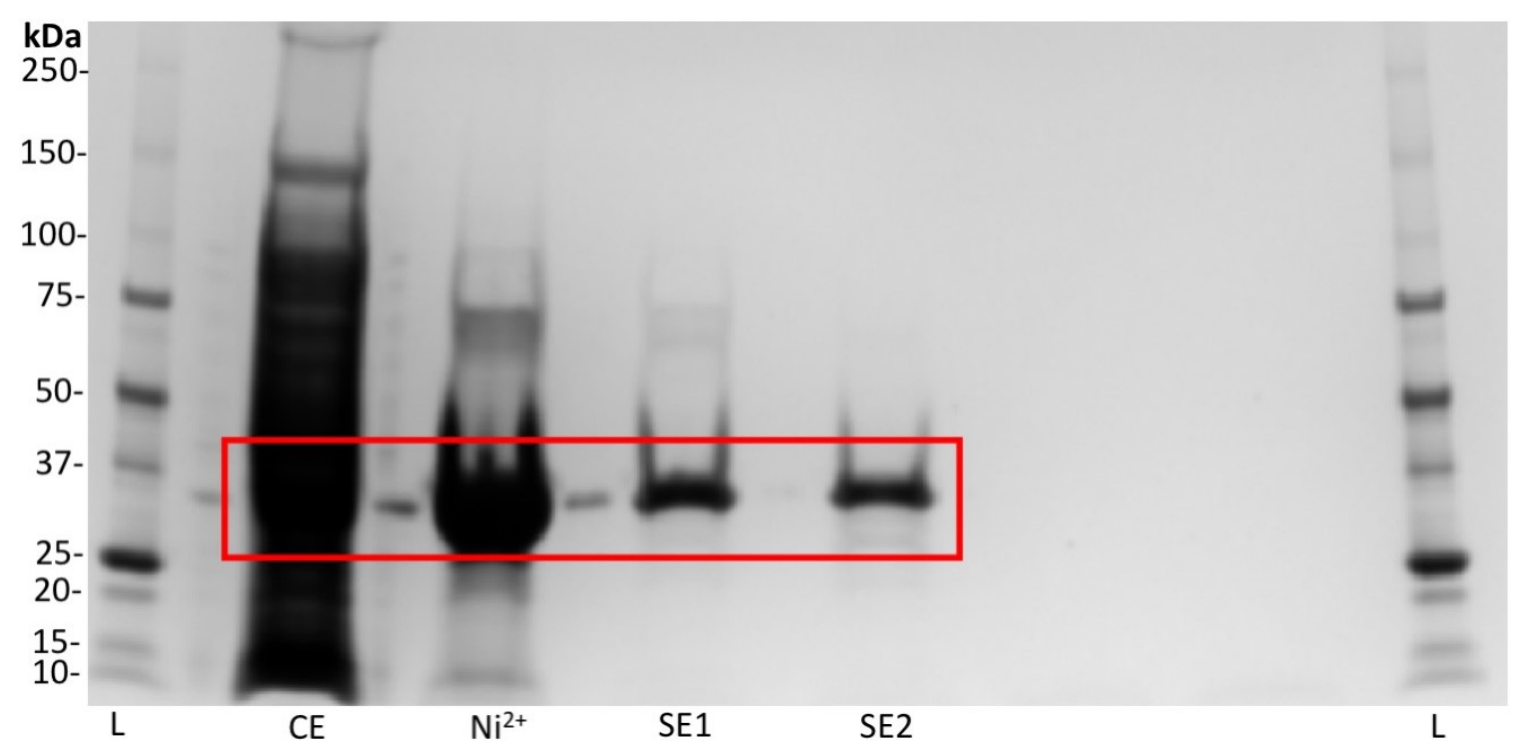

Figure 3-1. SDS-PAGE gel of CdFabK_15b purification results.

(A) Stain-free SDS-PAGE. L, ladder; CE, clarified extract from IPTG-induction growth; $\mathrm{Ni}^{2+}$, target fraction from nickel column; SE1, target fraction from beginning of size exclusion peak; SE2, target fraction from middle of size exclusion peak.
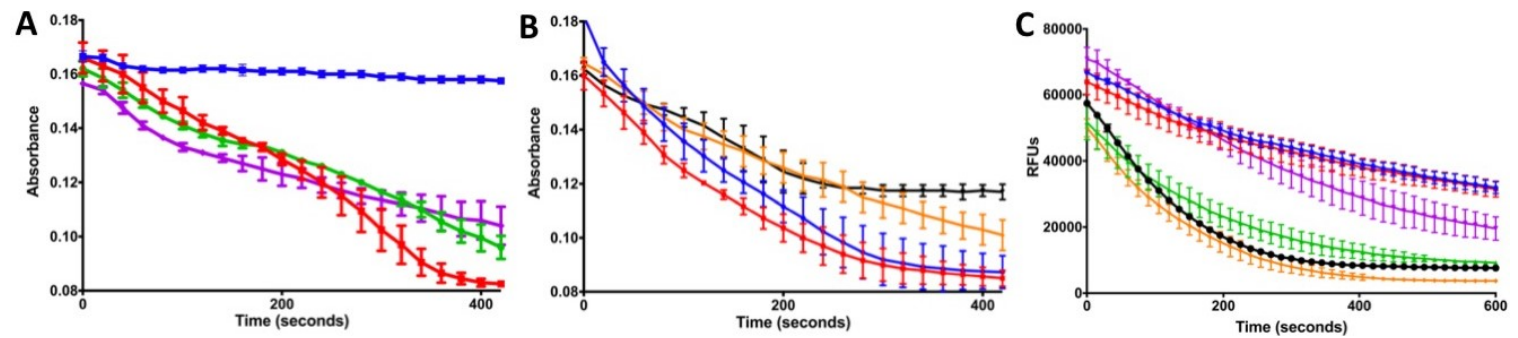

Figure 3-2. Enzyme activity studies of CdFabK_15b.

(A) CdFabK_15b stability trials measuring enzyme activity in different $\mathrm{NH}_{4} \mathrm{Cl}$ salt concentrations, $0 \mathrm{mM}$ (blue line with squares), $100 \mathrm{mM}$ (green line with dots), $300 \mathrm{mM}$ (purple line with diamonds), and $500 \mathrm{mM}$ (red line with squares). (B) CdFabK_15b stability trials measuring enzyme activity in different glycerol concentrations, $0 \%$ (black line with triangles), $4 \%$ (orange line with diamonds), $10 \%$ (red line with squares), and $15 \%$ (blue line with dots). (C) CdFabK_15b stability trials measuring enzyme activity in different buffers and at different $\mathrm{pH}$ values, MES pH 5.5 (blue line with dots), MES pH 6.5 (red line with squares), HEPES pH 7.5 (green line with triangles), HEPES pH 8.0 (black line with dots), HEPES pH 8.5 (orange line with diamonds), and CHES pH 9.5 (purple line with upside down triangles). All reactions were carried out in the presence of a fixed concentration of NADH $(150 \mu \mathrm{M})$ and Cro-CoA $(150 \mu \mathrm{M})$. 

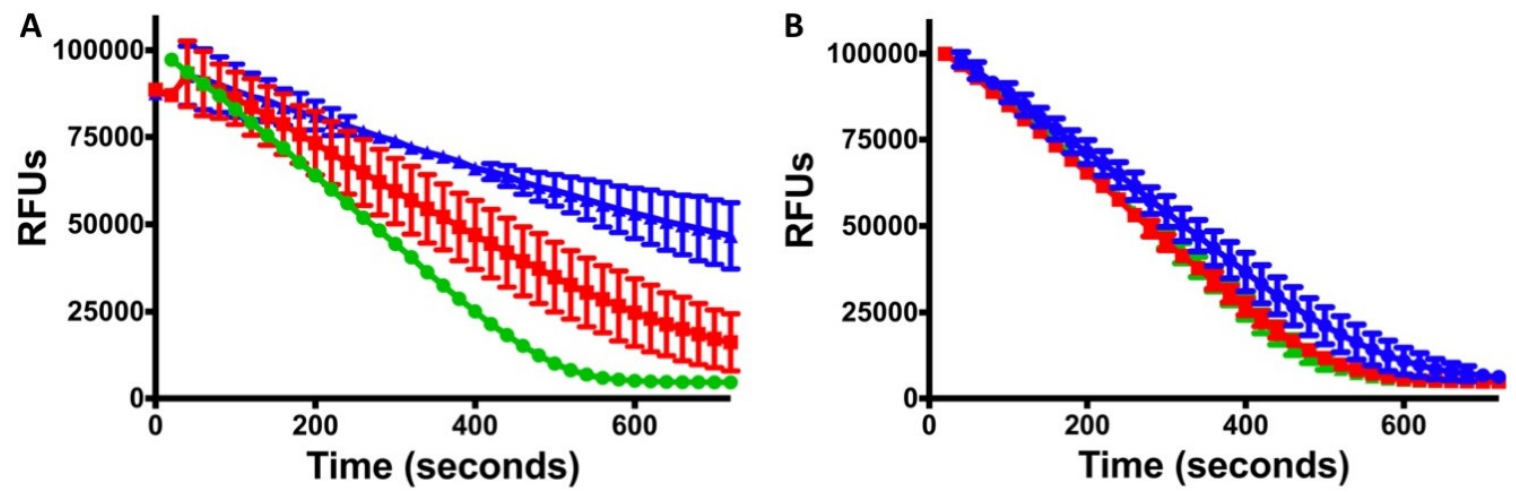

Figure 3-3. Enzyme dilution and stability studies of CdFabK_15b.

(A) CdFabK_15b stability trials measuring enzyme activity while diluted to $50 \mathrm{nM}$ at different incubation times 0 minutes (green line with circles), 30 minutes (red line with squares), and 60 minutes (blue line with triangles). (B) CdFabK_15b stability trials measuring enzyme activity while diluted to $50 \mathrm{nM}$ at different incubation times 0 minutes (green line with circles), 30 minutes (red line with squares), and 60 minutes (blue line with triangles) after having been diluted into a working stock including $2.5 \mathrm{mg} / \mathrm{mL}$ of $\gamma$ Globulins and an overall assay concentration of $0.125 \mathrm{mg} / \mathrm{mL} \gamma$-Globulins. All reactions were carried out in the presence of a fixed concentration of NADH $(150 \mu \mathrm{M})$ and CroCoA $(150 \mu \mathrm{M})$. 


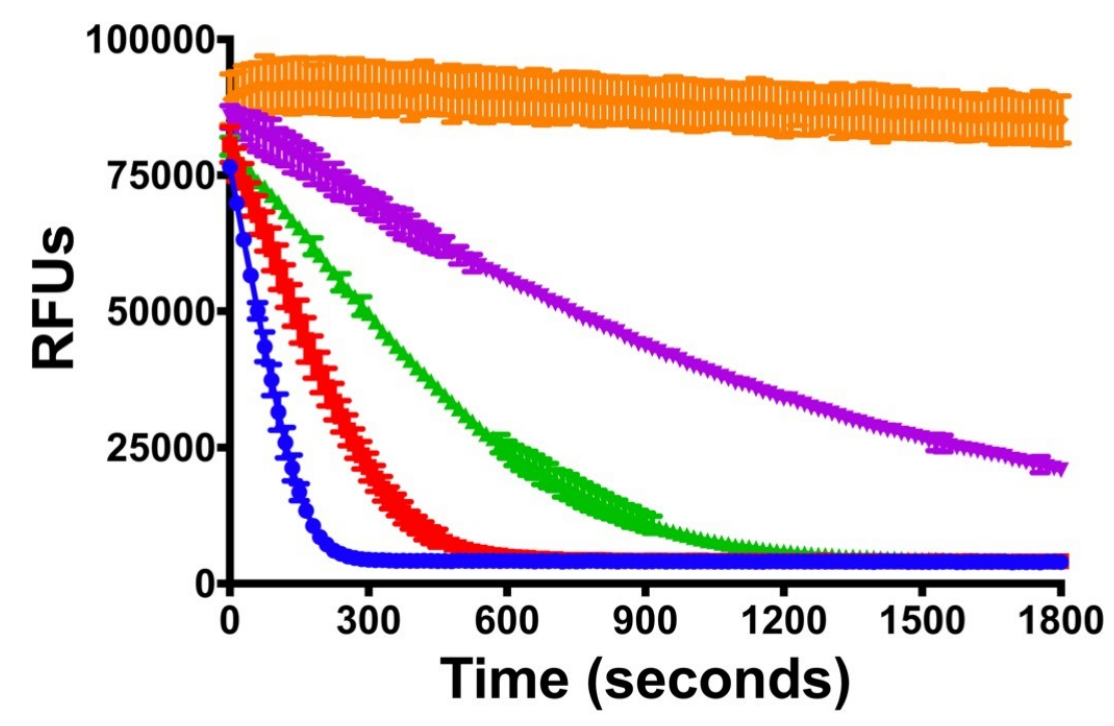

Figure 3-4. Extended enzyme dilution and stability studies of CdFabK_15b.

CdFabK_15b stability trials measuring enzyme activity at different two fold dilutions 50 $\mathrm{nM}$ (blue line with dots), $25 \mathrm{nM}$ (red line with squares), $12.5 \mathrm{nM}$ (green line with triangles), and $6.25 \mathrm{nM}$ (purple line with upside down triangles), and NADH control without enzyme (orange line with diamonds) after thirty minutes of incubation and a reaction runtime of thirty minutes. $\mathrm{CdFabK} \_15 \mathrm{~b}$ was diluted into a working stock including $5 \mathrm{mg} / \mathrm{mL}$ of $\gamma$-Globulins and an overall assay concentration of $0.25 \mathrm{mg} / \mathrm{mL} \gamma$ Globulins. All reactions were carried out in the presence of a fixed concentration of NADH $(150 \mu \mathrm{M})$ and Cro-CoA $(300 \mu \mathrm{M})$. 


\section{Crystallization of CdFabK_21d and CdFabK_15b}

Crystallization conditions were determined for both CdFabK_21d and CdFabK_15b (Figure 3-5). Protein crystals of CdFabK_21d were discovered first and diffracted to roughly $4 \AA$. CdFabK_15b crystallization conditions were also determined, and moderately optimized (as discussed in Materials and Methods) with the resulting crystals diffracting to roughly $3.5 \AA$.

\section{Discussion}

\section{CdFabK Production}

CdFabK was produced with two different hexa-Histidine tags, each to high homogeneity and purity (greater than 95\% via SDS-PAGE analysis) and over $10 \mathrm{mg}$ per liter of culture, however the C-term His-tagged construct proved highly unstable, despite significant efforts to optimize storage and activity assay conditions (data now shown). Therefore, the N-term His-tagged construct was expressed, purified via two-step purification, and studied further. Upon systematic stability and activity trial analysis, the salt, glycerol, and buffer and $\mathrm{pH}$ conditions were optimized for this specific construct as discussed below. While stored at $-80^{\circ} \mathrm{C}$, the activity of the enzyme remains well past six months, which is an important factor considering the desire to make large amounts of a single batch of enzyme, store it, and use it in the future for reliable and uniform and comparable high throughput compound screening.

\section{CdFabK Stabilization}

Upon systematic optimization of activity buffer conditions, optimal conditions were found to be $100 \mathrm{mM}$ HEPES $\mathrm{pH} 8.0,500 \mathrm{mM} \mathrm{NH}_{4} \mathrm{Cl}$, and $10 \%$ glycerol. This takes into account many considerations as the eventual goal of $\mathrm{CdFabK}$ production includes future assay development for compound screening and inhibitor studies. As such, certain factors of concern include, of course, target enzyme stability and activity during both benchtop experiments as well as after long-term storage. ${ }^{180}$ During analysis of the activity of the CdFabK_15b enzyme under diluted conditions generally conducive to creating working enzyme stock concentrations needed for compound screening, it was discovered that any significant dilution of CdFabK_15b below $\sim 100 \mu \mathrm{M}$ resulted in relatively rapid inactivation. Thus the addition of inert proteins were analyzed and the addition of $\gamma$ Globulins to the assay buffer for the dilution of the enzyme storage stock to an enzyme working stock was determined to greatly increase the overall stability of the enzyme with respect to the duration of activity. This allowed for substantial dilutions that would afford the opportunity to conduct assays in the presence of reasonable concentrations of DMSO and at low enough enzyme concentrations needed to screen inhibitors. 
A

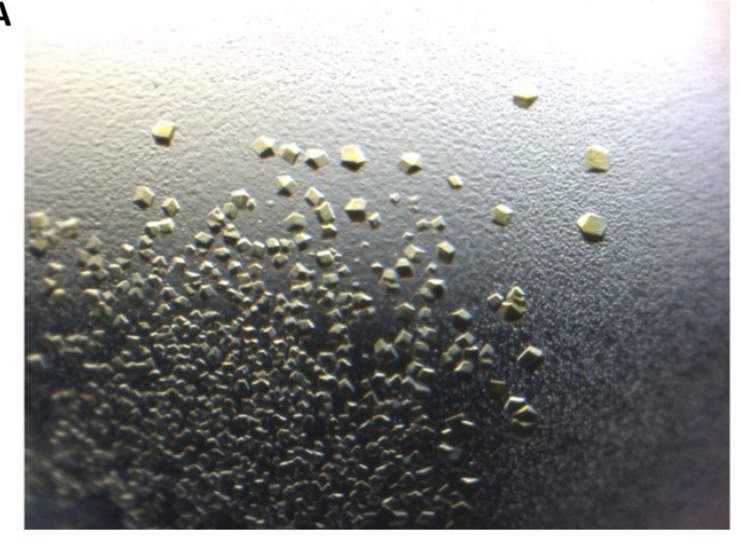

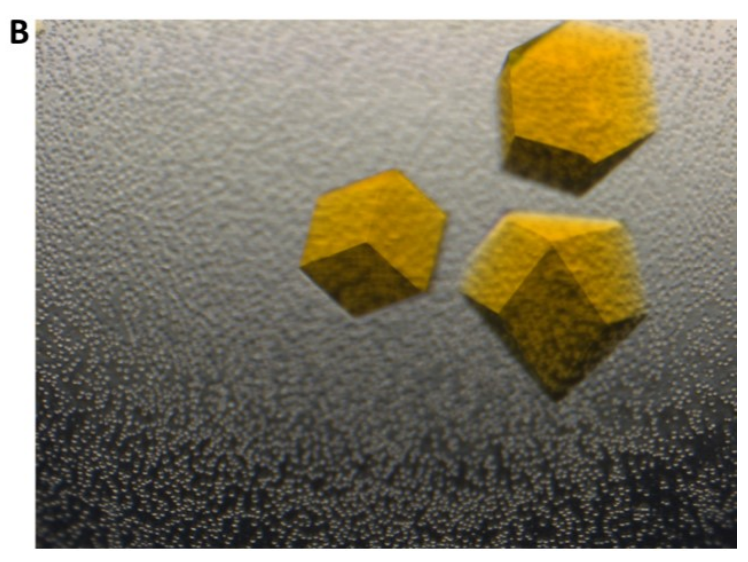

Figure 3-5. CdFabK crystals.

A. Original CdFabK_21d C-term hexa-His tagged crystals grown at $18^{\circ} \mathrm{C}$ from coarse Morpheus screen, condition 1-48 (0.12 M alcohols mix of 0.2 M 1,6-hexanediol, 0.2 M 1butanediol, 0.2 M 1,2-propanediol, 0.2 M 2-propanol, 0.2 M 1,4-butanediol, and 0.2 M 1,3-propanediol; 0.1 M buffer mix of Tris base and BICINE, $\mathrm{pH} \mathrm{8.5;} \mathrm{and} \mathrm{37.5 \%} \mathrm{v/v}$ precipitant mix of $25 \% \mathrm{v} / \mathrm{v} 2$-methyl-2,4-pentanediol, $25 \%$ polyethylene glycol 1000 , and $25 \%$ polyethylene glycol 3500). B. Optimized CdFabK_15b crystal grown at $18^{\circ} \mathrm{C}$ from coarse Morpheus screen, condition 2-48 (0.1 M amino acids mix of 0.2 M DL-glutamic acid monohydrate, 0.2 M DL-alanine, 0.2 M glycine, 0.2 M DL-lysine monohydrochloride, and 0.2 M DL-serine; $0.1 \mathrm{M}$ buffer mix of Tris base and BICINE, $\mathrm{pH} 8.5$; and $37.5 \% \mathrm{v} / \mathrm{v}$ precipitant mix of $25 \% \mathrm{v} / \mathrm{v} 2$-methyl-2,4-pentanediol, $25 \%$ polyethylene glycol 1000 , and $25 \%$ polyethylene glycol 3500 ) with $1: 1$ protein to $7 \% 10$ $\mathrm{mM}$ phenylimidazole compound $\mathbf{1 g}$ (see Chapter 4) and DMSO in Morpheus crystallization condition 2-48. 


\section{CdFabK Crystallization}

CdFabK required certain conditions for activity and stability, namely high salt, at least $300 \mathrm{mM} \mathrm{NH}_{4} \mathrm{Cl}$, and high glycerol, $18 \%$, not traditionally conducive to crystallization. As different methods were being considered for the optimization of crystallization standards, coarse crystallization trials were nonetheless attempted in the meantime. To some degree of surprise, crystallization conditions for both the C-term and $\mathrm{N}$-term His-tagged constructs were discovered, despite the high salt and high glycerol storage buffer conditions. While certainly a noteworthy accomplishment, the crystals continually resulted in lower, relatively weak diffraction (higher than $3.5 \AA$ ) and were therefore of little use in structure-guided drug development efforts.

Fine screening crystallization attempts were made to the best of in-lab abilities, but initial efforts to do so were met with difficulty. The crystallization conditions were very complex, including combinations of multiple precipitants like MPD, PEG 1000, and PEG 3350 for both CdFabK construct hit conditions, and different complex ligand combinations that include a mix of six different alcohols for the initial C-term hit condition, and a complex ligand mix of five different amino acids for the $\mathrm{N}$-term hit condition. Therefore, the realistic opportunity for fine screening was somewhat limited from the beginning and did not yield further results. As the crystals found off of the coarse screens were not shown to diffract near $2 \AA$, three-dimensional crystal structures have not been determined. Instead, additional crystallization and crystal optimization trials have continued.

Such trials have included apo- and co-crystallography efforts with CdFabK_15b and analogues of a known phenylimidazole FabK inhibitor compound. ${ }^{163}$ Additionally, CdFabK_15b has been screened against multiple other coarse crystal screens at various temperatures. Also, protein to crystallization condition ratios have been altered, as have drop sizes, and hanging vs. sitting drop methods have been employed. An Additive Screen (Hampton Research, Aliso Viejo, CA) has also been attempted, and different cryo conditions have been analyzed, including various PEG compounds, glycerol contents, sugars, oils, and so on. Various post-crystallization techniques reported in the literature, such as annealing and denaturing methods, have been extensively attempted as well. ${ }^{181}$ Thus far, diffraction has not improved, and further consideration is undergoing.

Though generally reported in the literature to have little effect on crystal structure quality and resolution, another consideration has been that the His-tag may interfere with tight crystal packing and resulting resolution. ${ }^{182}$ As such, attempts were made to cleave the His-tag off of CdFabK_15b via thrombin cleavage, but enzyme activity was completely lost and not recovered after doing so (data not shown). It is surmised that the reason for this is the extensive degree of dilution for extended amounts of time required for the thrombin cleavage reaction to occur. Additionally, preliminary attempts have been made to purify native, untagged $\mathrm{CdFabK}$ for eventual crystallization trials but, similar to overall CdFabK crystallization studies, progress is ongoing. 


\section{Summary}

Recent studies have shown that the fabk gene in $C$. difficile, which codes for the CdFabK enzyme, is essential, even in the presence of exogenous fatty acids. ${ }^{67}$ This alone supports $\mathrm{CdFabK}$ as a potentially promising novel antibacterial target. However, as those studies also show that a known inhibitor of $\mathrm{CdFabK}$ inhibits the growth of $C$. difficile without substantially inhibiting the growth of several selected representatives of the human microbiome, the promise and potential of $\mathrm{CdFabK}$ as a narrow-spectrum antibacterial target is also notably suggested. ${ }^{67}$ In order to further investigate CdFabK as a narrow spectrum target for antibacterial drug discovery, it was immediately necessary to produce pure, active, and stable target protein for further characterization and validation.

CdFabK initially proved to be a relatively unstable enzyme in that traditional purification buffers resulted in low-yield and inactive target protein. Via iterative trials, substantial yields of highly pure and active CdFabK are now rapidly obtainable.

Moreover, conditions for reproducible and reliable assaying of the target enzyme have been determined, including assay buffer conditions and long-term storage conditions. While the activity of the enzyme can now be reliably measured at a satisfactory $50 \mathrm{nM}$ conentration, the potential for measuring even lower concentrations-potentially into low double-digit nanomolar to single-digit nanomolar concentrations - appears possible. As this would be necessary for the testing of any potent $\mathrm{CdFabK}$ inhibitors discovered or developed, this is indeed a substantial and necessary achievement. Immediate future research, therefore, should include compound screening against $\mathrm{CdFabK}$, as well as enzymology and characterization of known FabK inhibitors (discussed further in Chapter 4).

Aside from producing pure, stable, and active enzyme for future compound screening, solving the three-dimensional CdFabK structure via X-ray crystallography is extremely important for future structure-guided drug discovery efforts. Unfortunately, the crystallography results reported herein did not include crystallization conditions or crystals that yielded the resolution needed to determine the structure of CdFabK at atomic or near-atomic resolution (near $2 \AA$ or lower), which would be necessary to observe structural characteristics in high detail and molecular interactions for potential ligands. Accordingly, future research should include continued crystallography efforts, both aimed at further optimizing diffraction of current crystals from known crystallization conditions, as well as determining new crystallization conditions and potentially constructing FabK mutants for additional crystallography studies (discussed further in Chapter 5). 


\section{CHAPTER 4. CDFABK ENZYMOLOGY AND MODALITY OF INHIBITION ${ }^{4}$}

\section{Introduction}

FabK from S. pneumoniae (SpFabK) was shown by Takahata et al. to be specifically inhibited by a compound (AG-205) they found during a high throughput screening campaign, which led to the development of the more potent phenylimidazolederived class of inhibitors via iterative medicinal chemistry modifications (e.g., replacing the amide linker with urea functionality, exploring various substitutions at the solvent exposed head region, un-fusing the benzimidazole and benzothiazole ring systems, and evaluating various substitutions at the tail region). ${ }^{166,168,183-185}$ Despite substantial gains in potency, further development was ceased, possibly due to the unfortunate fact that Streptococci are able to bypass FAS-II inhibition as they carry the FabT regulatory system. ${ }^{171}$ Because $C$. difficile harbors the FapR regulatory system, it presents an opportunity to renew the development of phenylimidazole FabK inhibitors. ${ }^{186}$ In an effort to characterize the enzyme target, essential kinetic evaluations of CdFabK, including substrate and cofactor $K_{\mathrm{m}}$ determinations, inhibition modality, and substrate inhibition effects are herein performed. Data is also reported showing phenylimidazoles display potent on-target biochemical activity against CdFabK (Table 4-1) and are amenable to modifications that improve physiochemical properties (Figure 4-1) while maintaining biochemical and anti-C. difficile activity.

As discussed above, the type II bacterial fatty acid synthesis pathway, FAS II, provides important fatty acid precursors for membrane phospholipids essential to bacterial cells and integral to sporulation. The FAS II pathway is distinctly separate from the mammalian FAS I pathway, which is composed of a single, multifunctional synthase, thus the FAS II enzymes represent novel and selective antibacterial targets that remain relatively unexploited. The structure and function of the enzymes in the bacterial FAS II pathway have been previously reviewed. ${ }^{169,187-189}$ As a metabolic pathway there is some concern about the ability of bacteria to bypass FAS II inhibition using exogenous fatty acids and this has been the subject of intense debate. ${ }^{171-173}$

Recent work, however, has demonstrated that certain bacteria, including $S$. aureus, remain susceptible to FAS II inhibition even in the presence of exogenous fatty acids due to differences in their regulation of genetic expression and feedback regulatory systems. ${ }^{71,190}$ As a rate-limiting enzyme in the FAS-II pathway, FabI, enoyl-[acyl-carrier protein (ACP)] reductase, represents a particularly attractive drug target and a number of inhibitors of this enzyme have been characterized. ${ }^{30,31,177,191-199}$ Triclosan, a stereotypical FabI inhibitor with a diphenyl ether scaffold, has been marketed for a number of years

\footnotetext{
${ }^{4}$ Portions of chapter from final submission adapted with permission from Jones, J. A.* et al. Smallmolecule inhibition of the C. difficile FAS-II enzyme, FabK, results in selective activity. ACS Chem Biol, https://doi.org/10.1021/acschembio.9b00293 (2019. In Press). (*Co-first author.) Copyright (2019) American Chemical Society. ${ }^{139}$
} 
Table 4-1. Inhibitory activity of Enoyl-ACP isozymes by phenylimidazole analogues.

\begin{tabular}{lcc}
\hline Compound & $\begin{array}{c}\text { CdFabK IC50 [95\% CI]; } \\
\text { \% Inhibition at 10 } \boldsymbol{M M}\end{array}$ & SpFabK IC50 [95\% CI] \\
\hline Triclosan & $>100 \mu \mathrm{M}$ & $>100 \mu \mathrm{M}$ \\
1a & $>10 \mu \mathrm{M} ; 38.51 \%$ & $0.730 \mu \mathrm{M}[0.434,1.456]$ \\
1b & $3.31 \mu \mathrm{M} ; 95 \% \mathrm{CI}[2.68,4.19]$ & $0.067 \mu \mathrm{M}[0.054,0.084]$ \\
1c & $>10 \mu \mathrm{M} ; 49.86 \%$ & $0.242 \mu \mathrm{M}[0.214,0.274]$ \\
1d & $>10 \mu \mathrm{M} ; 44.80 \%$ & $0.198 \mu \mathrm{M}[0.178,0.222]$ \\
1e & $2.86 \mu \mathrm{M}[2.02,4.35]$ & $0.078 \mu \mathrm{M}[0.066,0.093]$ \\
$\mathbf{1 f}$ & $4.63 \mu \mathrm{M}[3.12,8.02]$ & $0.163 \mu \mathrm{M}[0.124,0.214]$ \\
$\mathbf{1 g}$ & $7.35 \mu \mathrm{M}[5.35,11.65]$ & $0.085 \mu \mathrm{M}[0.072,0.101]$ \\
$\mathbf{1 h}$ & $>10 \mu \mathrm{M} ; 35.58 \%$ & $0.538 \mu \mathrm{M}[0.433,0.675]$ \\
AG-205 & $4.15 \mu \mathrm{M}[3.324,5.597]$ & $5.32 \mu \mathrm{M}[4.378,6.809]$ \\
\hline
\end{tabular}

CI, Confidence Interval

Reprinted from final submission with permission from Jones, J. A.* et al. Small-molecule inhibition of the C. difficile FAS-II enzyme, FabK, results in selective activity. ACS Chem Biol, https://doi.org/10.1021/acschembio.9b00293 (2019. In Press). (*Co-first author.) Copyright (2019) American Chemical Society. ${ }^{139}$

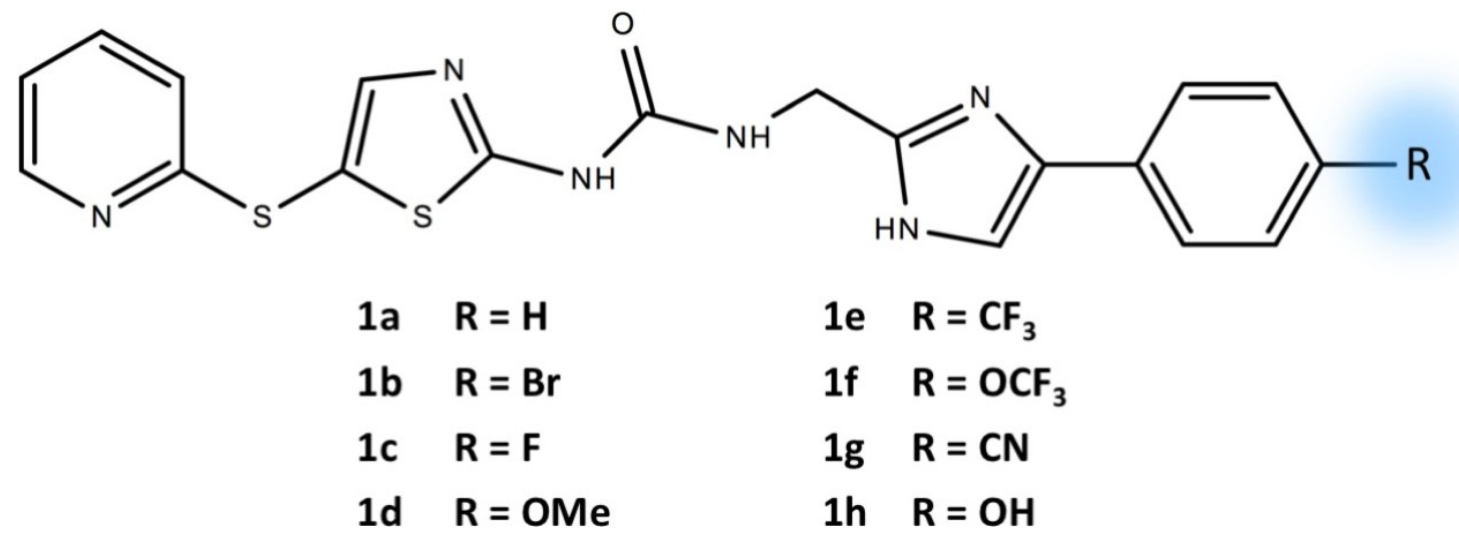

Figure 4-1. Phenylimidazole analogues 1a-h. 
and is used in a variety of over-the-counter household products as a sterilizing agent, however it has low utility for systemic use because of poor bioavailability. ${ }^{200,201}$

\begin{abstract}
Aims
While the fabk gene has been well-validated as an essential gene in $C$. difficile within the literature, the necessity to further validate the CdFabK enzyme as a druggable, selective antibacterial target still exists. ${ }^{67} \mathrm{In}$ an effort to do so, the following work details essential kinetic evaluations of $\mathrm{CdFabK}$, including substrate and cofactor $K_{\mathrm{m}}$ determinations; substrate inhibition effects; and inhibition studies, including modality data of phenylimidazole compounds and preliminary inhibition data of novel benzothiazole compounds against $\mathrm{CdFabK}$.
\end{abstract}

\title{
Materials and Methods
}

\section{CdFabK_15b Cloning, Expression, Purification}

The N-term hexa-histidine tagged CdFabK enzyme, CdFabK_15b, was produced and purified as detailed previously in Chapter 3 and used for all CdFabK enzymology, kinetic, and inhibitor studies discussed in this chapter.

\section{SpFabK Cloning and Plasmid Construction}

The fabk gene from S. pneumo strain R6 was cloned and amplified using standard PCR protocol and restriction enzyme techniques. Primers were designed around the NdeI and BamHI restriction sites using the following primers:

$$
\text { SpFabKfwd: 5'-GGAGGCATATGAAAACGCGTATTAC-3' }
$$

$$
\text { SpFabKrev: 5'-AAGGATCCTTAGTCATTTCTTACAACTCC -3' }
$$

The target gene was cloned into the pET15b vector and transformed into BL21-Gold (DE3) cells (Agilent Technologies, Santa Clara, CA). The protein was overexpressed in E. coli BL21-Gold (DE3) cells via cultivation at $37^{\circ} \mathrm{C}$ in TB medium with $100 \mu \mathrm{g} / \mathrm{mL}$ ampicillin by first transferring $1 \%(\mathrm{v} / \mathrm{v})$ overnight culture into fresh medium. Cells were allowed to grow until $\mathrm{OD}_{600}$ of $\sim 0.6$ and the protein expression was induced with 0.1 mM IPTG. Cells were allowed to grow further for 18 hours at $18^{\circ} \mathrm{C}$ and the cells were harvested by centrifugation at $18000 \mathrm{x} g$ for 15 minutes. After induction and growth, the cells were harvested by centrifugation at $18000 \mathrm{x}$ g for 15 minutes. Pellets were stored at $-80^{\circ} \mathrm{C}$ until use. 


\section{SpFabK_15b Purification}

The cell pellet was resuspended in $50 \mathrm{~mL}$ SpFabK Lysis Buffer (CdFabK Buffer A with $0.5 \mathrm{mg} / \mathrm{mL}$ lysozyme, $1 \mathrm{mg} / 100 \mathrm{~mL}$ DNase, $5 \mathrm{mM} \mathrm{MgCl} 2,0.5 \%$ Triton-X, and 1 Pierce EDTA-free Protease Inhibitor tablet per $50 \mathrm{~mL}$ ) and lysed at $4^{\circ} \mathrm{C}$ with gentle stirring, then sonicated on ice at $50 \%$ amplitude for eight minutes, with eight seconds on and 24 seconds off. The cell lysates were centrifuged at $39000 \mathrm{x}$ g for $15 \mathrm{~min}$ and the supernatant was passed through a $0.22 \mu \mathrm{M}$ PES syringe filter (EMD Millipore, Billerica, $\mathrm{MA})$. N-term hexa-histidine tagged SpFabK (SpFabK_15b) was purified via immobilized metal affinity chromatography (IMAC) by loading the filtered lysate on a HisTrap HP column (GE Life Sciences) in SpFabK Buffer A, washed over 20 column volumes with SpFabK Buffer A, and eluted via linear gradient to $100 \%$ SpFabK Buffer B (SpFabK Buffer A with $500 \mathrm{mM}$ imidazole) over 20 column volumes. After IMAC purification, fractions were pooled and further purified by size exclusion chromatography (SEC) on a HiLoad 26/600 Superdex 200 PG size exclusion column (GE Healthcare Life Sciences, Pittsburgh, PA) in SpFabK SEC Buffer (50 mM Tris pH 7.4, $100 \mathrm{mM} \mathrm{NH}_{4} \mathrm{Cl}$, and $3 \mathrm{mM}$ DTT) via isocratic elution. The enzyme was confirmed via PAGE gel analysis and the enzyme activity was analyzed in an Implen NP80 nanophotometer as described below (West Lake Village, CA). The protein was concentrated to $75 \mu \mathrm{M}$ with $35 \%$ overall glycerol and stored at $-80^{\circ} \mathrm{C}$ until use.

\section{SaFabI_15b Expression}

FabI from S. aureus cloned into the pET15b vector (SaFabI_15b) was transformed into BL21-Gold (DE3) cells (Agilent Technologies, Santa Clara, CA) previously (data not shown), and SaFabI_15b was produced in high yield and purity as reported elsewhere in the literature with minor modifications. ${ }^{194}$ Briefly, SaFabI_15b was overexpressed at $37^{\circ} \mathrm{C}$ in $\mathrm{TB}$ medium with $100 \mu \mathrm{g} / \mathrm{mL}$ ampicillin by transferring $1 \%$ $(\mathrm{v} / \mathrm{v})$ overnight culture into fresh medium. Cells were allowed to grow until they reached an $\mathrm{OD}_{600}$ of $\sim 0.6$ and the protein expression was induced with $0.5 \mathrm{mM}$ IPTG grown further for 4 hours at $37^{\circ} \mathrm{C}$. After induction and growth, the cells were harvested by centrifugation at $18000 \mathrm{xg}$ for 15 minutes. Pellets were stored at $-80^{\circ} \mathrm{C}$ until use.

\section{SaFabI_15b Purification}

The cell pellet was resuspended in $50 \mathrm{~mL}$ SaFabI Buffer A ( $500 \mathrm{mM}$ Tris $\mathrm{pH} 8.0$, $500 \mathrm{mM} \mathrm{NaCl}$, and $10 \mathrm{mM}$ imidazole) with $0.5 \mathrm{mg} / \mathrm{mL}$ lysozyme, $1 \mathrm{mg} / 100 \mathrm{~mL}$ DNase, 5 $\mathrm{mM} \mathrm{MgCl} 2,0.5 \%$ Triton-X, and 1 Pierce EDTA-free Protease Inhibitor tablet per $50 \mathrm{~mL}$, and lysed at $4{ }^{\circ} \mathrm{C}$ with gentle stirring, then sonicated on ice at $50 \%$ amplitude for eight minutes, with eight seconds on and 24 seconds off. The cell lysate was centrifuged at $39000 \mathrm{x}$ g for 15 minutes and the supernatant was passed through a $0.22 \mu \mathrm{M}$ PES filter (EMD Millipore, Billerica, MA). The target protein purification was performed by affinity chromatography on HisTrap HP column (GE Lifesciences) with SaFabI Buffer A as binding and wash buffer and SaFabI Buffer B (SaFabI Buffer A with $500 \mathrm{mM}$ 
imidazole) as elution buffer. Elution peaks were pooled and buffer exchanged in a 10,000 MWCO Amicon ${ }^{\mathrm{TM}}$ Ultra-15 Centrifugal Filter Unit from EMD Millipore ${ }^{\mathbb{B}}$ (Billerica, MA) into SaFabI Storage Buffer (50 mM MES pH 5.5, $100 \mathrm{mM} \mathrm{NaCl}, 100$ mM EDTA).

SaFabI_15b was confirmed via PAGE gel analysis and the enzyme activity was confirmed in an Implen NP80 nanophotometer as described below (West Lake Village, CA).

\section{FAS-II Biochemical Enzyme Assays}

All compounds were dissolved in DMSO at concentrations of $10 \mathrm{mM}$, then further diluted in pure DMSO to the required concentrations. Both CdFabK and SpFabK assays were conducted via the following protocol: Reactions were carried out at $25^{\circ} \mathrm{C}$ in FabK Assay Buffer (100 mM HEPES pH 8.0, $500 \mathrm{mM} \mathrm{NH}_{4} \mathrm{Cl}, 10 \%$ glycerol, and 0.125 $\mathrm{mg} / \mathrm{mL} \gamma$-Globulins; $10 \%$ DMSO) with $150 \mu \mathrm{M}$ Cro-CoA and $150 \mu \mathrm{M} \mathrm{NADH.}$ Compounds were incubated in three-fold dilutions (ranging from $100 \mu \mathrm{M}$ to $5 \mathrm{nM}$ for $\mathrm{CdFabK}$ and $33 \mu \mathrm{M}$ to $1.7 \mathrm{nM}$ for $\mathrm{SpFabK}$ ) in the presence of $50 \mathrm{nM}$ target enzyme. Compounds and enzyme were incubated together for 10 minutes before the Cro-CoA substrate was added and the reaction was started immediately afterward via the addition of NADH. NADH fluorescence ( $340 \mathrm{~nm} / 460 \mathrm{~nm}$ ) was measured in a Biotek Synergy H1 microplate reader (Winooski, VT) in a final volume of $100 \mu \mathrm{L}$ in Greiner Bio-One ${ }^{\mathrm{TM}}$ 384-Well $\mu$ Clear $^{\mathrm{TM}}$ Bottom Polystyrene Microplates (Monroe, NC) via 20 second intervals for 10 minutes to evaluate the rate of reaction.

SaFabI assays were conducted at $25^{\circ} \mathrm{C}$ in FabI Assay Buffer (50 mM MES pH $5.5,150 \mathrm{mM} \mathrm{NaCl}, 10 \mathrm{mM}$ EDTA, and 2\% DMSO) with $300 \mu \mathrm{M}$ NADPH and $1 \mathrm{mM}$ Cro-CoA. Compounds were incubated at $10 \mu \mathrm{M}$ with $500 \mathrm{nM}$ SaFabI for 20 minutes. Reactions were started via the addition of NADPH. NADPH absorbance was measured $(340 \mathrm{~nm})$ every 10 seconds for 10 minutes in a final volume of $100 \mu \mathrm{L}$ to evaluate the rate of reaction.

\section{FabK IC50 Calculations and Kinetics}

Starting at three minutes, linear slopes were measured for three additional minutes and used to determine the reaction rates. Measurements were conducted in duplicate and $\mathrm{IC}_{50}$ s were calculated via GraphPad Prism 7.0d (La Jolla, CA) using four-parameter logistic (Hill) curve analysis using the equation $\mathrm{Y}=$ Bottom $+($ Top Bottom $) /\left[1+10^{\wedge}((\operatorname{LogIC} 50-\mathrm{X}) *\right.$ HillSlope $\left.)\right]$, where $\mathrm{X}$ is logarithm of dose and $\mathrm{Y}$ is response. Kinetics with respect to cofactor and substrates were also assessed via GraphPad Prism 7.0d comparing both standard Michaelis-Menten and substrate inhibition models. 


\section{FabI Activity}

Starting at two minutes, linear slopes were measured for three minutes and used to determine the reaction rates. Measurements were conducted in duplicate and percent inhibitions were calculated against un-inhibited enzyme.

\section{Generation of SpFabK and CdFabK Sequence Alignment}

SpFabK and CdFabK alignment figure generated with ESPript 3 server (http://espript.ibcp.fr) using multiple protein sequence alignment from Clustal Omega and secondary structure information from SpFabK structure (PDB 2Z6I). ${ }^{119,122}$

\section{Results}

\section{The CdFabK Enzyme Possesses Intrinsic NADH Oxidative Activity and Double Substrate Inhibition}

The CdFabK enzyme displayed NADH oxidative activity in the absence of enoyl substrate (Figure 4-2). Apparent $K_{\mathrm{m}}\left(K_{\mathrm{m}}{ }^{\text {app }}\right)$ values were determined via 1.5-fold serial dilutions of the NADH cofactor while holding the enoyl substrate, crotonyl coenzyme A (Cro-CoA), constant at $150 \mu \mathrm{M}$; likewise, 1.5-fold serial dilutions of Cro-CoA were tested while holding the NADH cofactor constant at $150 \mu \mathrm{M}$; and an alternative enoyl substrate, octenoyl coenzyme A (Oct-CoA), was tested similarly via 1.5-fold dilutions while holding NADH constant at $150 \mu \mathrm{M}$. Initially, $K_{\mathrm{m}}{ }^{\text {app }}$ values were determined via standard Michaelis-Menten non-linear regression fits. However, upon further analysis, the data for cofactor and substrates all fit a substrate inhibition model $\left(\mathrm{R}^{2}=0.9854\right.$; 0.7607; and 0.8479, respectively) better than the poorly fit standard Michaelis-Menten models. As such, respective $K_{\mathrm{m}}{ }^{\text {app }}$ values for NADH, Cro-CoA, and octenoyl coenzyme A were determined to be $138.1 \mu \mathrm{M}, 327.0 \mu \mathrm{M}$, and $420.8 \mu \mathrm{M}$, as opposed to $23.0 \mu \mathrm{M}$, $176.0 \mu \mathrm{M}$, and $65.5 \mu \mathrm{M}$ observed with the standard Michaelis-Menten model, respectively.

\section{Phenylimidazole-Derived Compounds Selectively Inhibit the $C$. difficile FabK Enzyme}

Phenylimidazole compounds have been shown to inhibit SpFabK (PDB 2Z6J) and lack inhibitory activity against FabI from E. coli. ${ }^{183,184}$ To evaluate activity against $\mathrm{CdFabK}$, phenylimidazole compounds were screened against purified enzyme to determine percent inhibition at $10 \mu \mathrm{M}$. Compounds yielding over $50 \%$ inhibition were further screened to determine on-target $50 \%$ inhibitory concentrations $\left(\mathrm{IC}_{50}\right)$. Compounds were also screened at $10 \mu \mathrm{M}$ against purified FabI from Staphylococcus aureus (SaFabI) to directly compare activity profiles. 

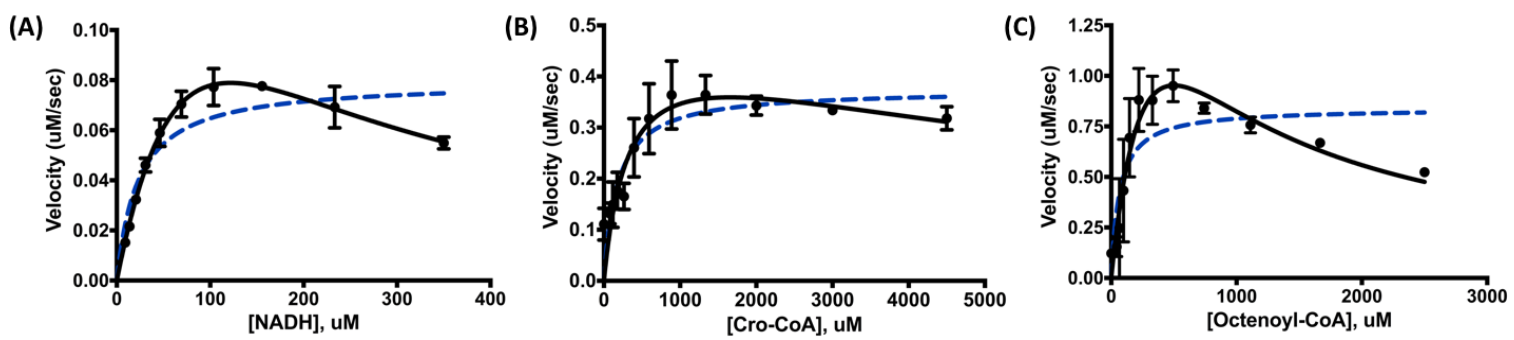

Figure 4-2. Enzyme kinetics of CdFabK vs. velocity.

(A) Non-linear regression curve fit of velocity of $\mathrm{CdFabK}$ with varying concentrations of NADH (1.5-fold dilutions from $350 \mu \mathrm{M}$ to $9.1 \mu \mathrm{M})$ at a fixed concentration of Cro-CoA $(325 \mu \mathrm{M})$ demonstrating NADH substrate inhibition. Standard Michaelis-Menten fit (dashed blue line) and substrate inhibition line (solid black line with dots) shown for comparison. (B) Non-linear regression curve fit of velocity of $\mathrm{CdFabK}$ with varying concentrations of enoyl substrate Cro-CoA (1.5-fold dilutions from $4500 \mu \mathrm{M}$ to $78 \mu \mathrm{M}$ and $0 \mu \mathrm{M})$ at a fixed concentration of NADH $(125 \mu \mathrm{M})$ demonstrating Cro-CoA substrate inhibition. (C) Non-linear regression curve fit of velocity of CdFabK with varying concentrations of alternative enoyl substrate octenoyl-CoA (1.5-fold dilutions from $2500 \mu \mathrm{M}$ to $43 \mu \mathrm{M}$ and $0 \mu \mathrm{M})$ at a fixed concentration of NADH (125 $\mu \mathrm{M})$ demonstrating octenoyl-CoA substrate inhibition.

Reprinted from final submission with permission from Jones, J. A.* et al. Small-molecule inhibition of the C. difficile FAS-II enzyme, FabK, results in selective activity. $A C S$ Chem Biol, https://doi.org/10.1021/acschembio.9b00293. (2019. In Press). (*Co-first author.) Copyright (2019) American Chemical Society. ${ }^{139}$ 
We confirmed the phenylimidazole compounds inhibit CdFabK in a dosedependent manner. All compounds except 1a, 1c, 1d, and $\mathbf{1 h}$ showed greater than $50 \%$ inhibition of $\mathrm{CdFabK}$ at $10 \mu \mathrm{M}$. $\mathrm{IC}_{50 \mathrm{~S}}$ ranged from $7.35 \mu \mathrm{M}$ with $\mathbf{1 g}$ to $2.86 \mu \mathrm{M}$ with $\mathbf{1 e}$, with the series prototype, $\mathbf{1 b}$, showing an $\mathrm{IC}_{50}$ of $3.31 \mu \mathrm{M}$. Resulting Hill coefficients from $\mathrm{IC}_{50}$ logistic curve fits for $\mathbf{1 b}, \mathbf{1 e}$, and $\mathbf{1 g}$ were 0.901 (95\% Confidence Interval (CI) $=0.761-1.064), 0.8594(95 \% \mathrm{CI}=0.6449-1.134)$, and $1.014(95 \% \mathrm{CI}=0.7417-1.365)$, respectively. These values are not significantly different from unity (Hill slope of 1.000), suggesting these compounds display normal inhibitory behavior and do not bind cooperatively, aggregate, form micelles, or demonstrate problematic insolubility. It also indicates the enzyme's active sites function independently, despite CdFabK being a functional dimer. ${ }^{163,202}$

All compounds showed $\mathrm{IC}_{50} \mathrm{~S}$ against $\mathrm{SpFabK}$ in the sub-micromolar range, with 1 b resulting in an $\mathrm{IC}_{50}$ of $67 \mathrm{nM}$, roughly 49 -fold better as compared to CdFabK. Likewise, $1 \mathrm{e}$ and $\mathbf{1 g}$ resulted in respective $\mathrm{IC}_{50} \mathrm{~S}$ of 78 and $85 \mathrm{nM}$ against $\mathrm{SpFabK}$, illustrating roughly 87 - and 37 -fold better respective $\mathrm{IC}_{50} \mathrm{~S}$ as compared to $\mathrm{CdFabK}$. While activity of $\mathbf{1 b}$ against SpFabK was more potent than against CdFabK, it was still roughly 30 times less potent against $\mathrm{SpFabK}$ in our hands than reported elsewhere. ${ }^{184} \mathrm{As}$ the monomer enzyme concentration used here $(50 \mathrm{nM})$ was lower than that used in previous reports (about $60 \mathrm{nM}$ ), the reason for this discrepancy is not readily apparent. FabI was confirmed to be resistant to the phenylimidazole compounds as both $\mathbf{1 b}$ and AG-205 showed little inhibitory effect against SaFabI with percent inhibition values well below $50 \%$ (22.40 and $18.22 \%$, respectively) at $10 \mu \mathrm{M}$.

The target specificity profile of CdFabK was examined via activity in the presence of triclosan - a well-known, potent, slow-binding inhibitor of the FabI isozyme. ${ }^{203}$ To verify our own methods, triclosan was confirmed to be a potent inhibitor of SaFabI, with nearly $90 \%$ inhibition at $10 \mu \mathrm{M}$. Like SpFabK, CdFabK proved to be resistant to triclosan at $100 \mu \mathrm{M}$ and $\mathrm{IC}_{50}$ values were not determined.

\section{Phenylimidazole Compound $1 \mathrm{~b}$ is Competitive for NADH and Uncompetitive for the Enoyl Substrate Against CdFabK}

To elucidate the modality of inhibition of the phenylimidazole compounds against $\mathrm{CdFabK}$, we analyzed the inhibitory activity of $\mathbf{1 b}$ against purified enzyme in the presence of varying concentrations of NADH while holding Cro-CoA constant, and likewise in the presence of varying concentrations of Cro-CoA while holding NADH constant. Both non-linear fits and Lineweaver-Burk plots were analyzed. The best nonlinear fits for each substrate suggest that $\mathbf{1 b}$ acts as a competitive inhibitor of $\mathrm{CdFabK}$ with respect to NADH $\left(\mathrm{R}^{2}=0.91\right)$ and an uncompetitive inhibitor with respect to CroCoA $\left(\mathrm{R}^{2}=0.90\right)$. The Lineweaver-Burk plots for each substrate corroborate these findings (Figure 4-3). 

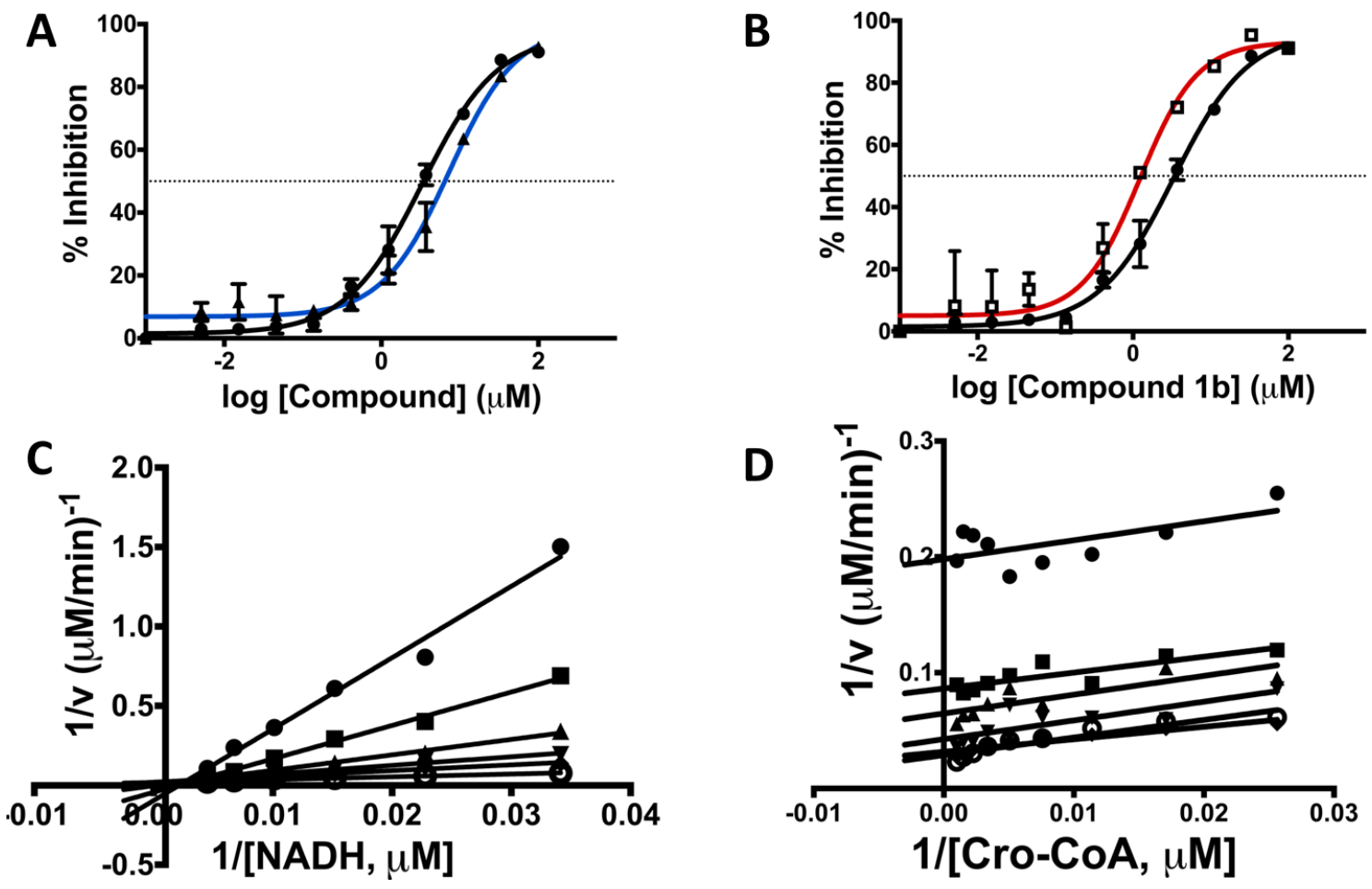

Figure 4-3. Characterization of inhibitory activity and mechanism of $1 \mathrm{~b}$ against CdFabK.

(A) Sigmoidal plots demonstrating dose response curves for the inhibition of CdFabK by two different phenylimidazole analogues, $1 \mathrm{~b}$ (black line with dots) and $1 \mathrm{~g}$ (blue line with triangles). (B) Sigmoidal plots as dose response curves for the inhibition of CdFabK by $1 \mathrm{~b}$ with different concentrations of Cro-CoA, $150 \mu \mathrm{M}$ Cro-CoA (black line with dots) and $325 \mu \mathrm{M}$ Cro-CoA (red line with squares). (C) Lineweaver-Burk plot showing competitive inhibition of CdFabK binding NADH by $1 \mathrm{~b}$. CdFabK was incubated with varying concentrations of $1 \mathrm{~b}$ and $\mathrm{NADH}$ at a fixed concentration of Cro-CoA $(325 \mu \mathrm{M})$. Concentrations of $1 \mathrm{~b}$ were $33 \mu \mathrm{M}$ (closed circle), $11 \mu \mathrm{M}$ (square), $3.67 \mu \mathrm{M}$ (triangle), $1.22 \mu \mathrm{M}$ (upside-down triangle), $0.4 \mu \mathrm{M}$ (diamond), and $0 \mu \mathrm{M}$ (open circle). (D) Lineweaver-Burk plot showing uncompetitive inhibition of CdFabK binding Cro-CoA by 1b. CdFabK was incubated with varying concentrations of $1 \mathrm{~b}$ and Cro-CoA at a fixed concentration of NADH $(150 \mu \mathrm{M})$. Concentrations of $1 \mathrm{~b}$ were $33 \mu \mathrm{M}$ (closed circle), 11 $\mu \mathrm{M}$ (square), $3.67 \mu \mathrm{M}$ (triangle), $1.22 \mu \mathrm{M}$ (upside-down triangle), $0.4 \mu \mathrm{M}$ (diamond), and $0 \mu \mathrm{M}$ (open circle).

Reprinted from final submission with permission from Jones, J. A.* et al. Small-molecule inhibition of the C. difficile FAS-II enzyme, FabK, results in selective activity. ACS Chem Biol, https://doi.org/10.1021/acschembio.9b00293. (2019, In Press). (*Co-first author.) Copyright (2019) American Chemical Society. ${ }^{139}$ 


\section{Benzothiazole Compound KH-70 Potently Inhibits CdFabK}

Benzothiazole compounds have also been shown to inhibit SpFabK. ${ }^{167}$ Considering the lack of a significant difference between the observable potencies of phenylimidazoles and the prototype benzothiazole compound AG-205 against CdFabK, benzothiazole analogues were further considered. A hit determined from a virtual screening shape matching campaign conducted by the principal investigator of the laboratory resulted in a benzothiazole analogue, KH-70, that was later screened under the same optimized Km conditions as $\mathbf{1 b}$ (Materials and Methods). KH-70 proved to be the most potent inhibitor of CdFabK to date, with an $\mathrm{IC}_{50}$ of $0.3545 \mu \mathrm{M}(95 \% \mathrm{CI} 0.2509$ to 0.4923) (Figure 4-4)

\section{Discussion}

We report here the kinetic evaluation of CdFabK, the biochemical activity of a series of phenylimidazole analogues, and microbiological data suggesting these compounds' selective antibacterial activity against $C$. difficile over several other prominent gut organisms. The compounds display promising, low micromolar $\mathrm{CdFabK}$ inhibitory activity without significantly affecting the in vitro growth of important gut organisms. CdFabK biochemical activity assays confirmed the inhibitory activity of all phenylimidazole analogues against $\mathrm{CdFabK}$, with several showing potent, low micromolar activity. This data further demonstrates the enzyme's druggability and the potential of the phenylimidazole compounds as leads for developing a novel series of narrow-spectrum anti- $C$. difficile drug candidates. As the imidazole and thiazole-urea moieties in the first-generation inhibitor $\mathbf{1 b}$ were predicted in modeling studies to establish three hydrogen bond interactions, and thus play a significant role in FabK binding, initial structure activity relationship (SAR) and physicochemical exploration during druggability validation was focused on the 4-bromo-phenyl region of the molecule (Figure 4-1). The calculated solubility of $\mathbf{1 b}$ is poor (cLogP 4.56). Therefore, in this work, a new expanded set of substituents with different lipophilic and electronic properties in the tail region were tested to further probe existing SAR as well as enhance solubility. Specifically, six new compounds $\mathbf{1 c - 1 h}$, along with two known compounds 1ab, were evaluated and, while chemical modifications did not substantially increase potency, $\mathrm{IC}_{50}$ values against $\mathrm{CdFabK}$ indicate inhibitory activity was not abolished despite crucial solubility enhancement via modification at this region. This is illustrated by comparing $\mathbf{1 b}$ (4-bromophenyl, cLogP 4.56 and $\mathrm{IC}_{50} 3.31 \mu \mathrm{M}$ ) and $\mathbf{1 g}$ (4cyanophenyl, significantly improved cLogP 3.15, similar IC $507.35 \mu \mathrm{M}$ ). Because the target enzyme's natural, in vivo function involves the binding of relatively lipophilic fatty acid precursor substrates, this was a noteworthy discovery that will serve as an important structural basis for future inhibitor design and synthesis. While 1e and 1f showed MIC values similar in potency to $\mathbf{1 b}$ and $\mathbf{1 g}$, increased cLogP values (4.6 and 4.8, respectively) for both compounds preclude them from further assessment.

A high degree of primary sequence identity and active site similarity exists between $\mathrm{CdFabK}$ and $\mathrm{SpFabK}$ (58\% overall identity, 74\% overall similarity, and 1\% gaps 
A<smiles>CS(=O)(=O)c1ccc2nc(NC(=O)Cc3csc(-n4cccc4)n3)sc2c1</smiles>

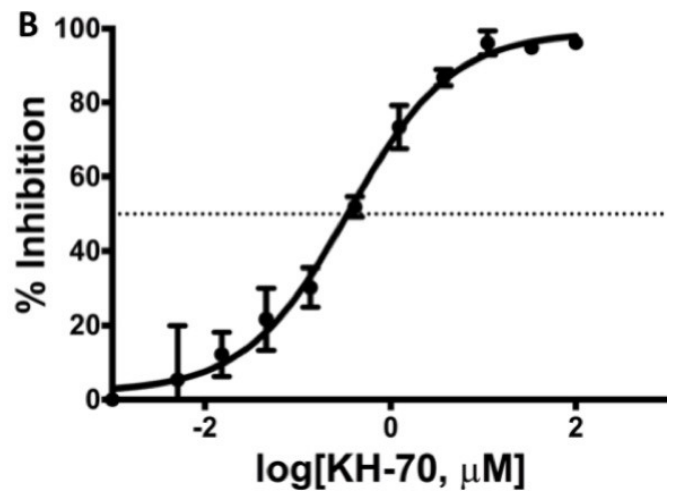

Figure 4-4. Structure and dose response curve against CdFabK for the novel hit KH-70.

(A) Structure of benzothiazole hit KH-70. (B) Sigmoidal plot demonstrating dose response curve for the inhibition of $\mathrm{CdFabK}$ by $\mathrm{KH}-70$. 
with 2 missing loops on SpFabK, PDB 2Z6J100\% and 91\% active site identity within $3 \AA$ and $4 \AA$ from bound inhibitors, respectively; Figure 4-5). Despite this, the phenylimidazole compounds showed greater activity against SpFabK than CdFabK (submicromolar vs. low micromolar, respectively). The compounds, along with triclosan, were tested against both FabK and FabI to determine comparative FAS-II inhibition profiles. The compounds potently inhibited FabK but lacked activity against FabI, while triclosan potently inhibited FabI but lacked activity against FabK. The reason for the potency disparity of phenylimidazoles against the two similar FabK enzymes is not readily apparent. The mode of inhibition for the phenylimidazoles was reported to be competitive inhibition of SpFabK binding NADH and uncompetitive of SpFabK binding Cro-CoA, ${ }^{163}$ and confirmed here to be the same against CdFabK (Figure 4-2) and therefore not the cause for potency dissimilarity. Evaluation at incorrect $K_{\mathrm{m}}{ }^{\text {app }}$ values of substrate and cofactor was another potential cause for dissimilar potencies, therefore a substrate inhibition model was considered, increasing the $K_{\mathrm{m}}{ }^{\text {app }}$ of Cro-CoA to $327 \mu \mathrm{M}$, up from $176 \mu \mathrm{M}$ seen with a standard Michaelis-Menten model. After re-testing $\mathbf{1 b}$ against CdFabK with $325 \mu \mathrm{M}$ Cro-CoA, the $\mathrm{IC}_{50}$ dropped from $3.31 \mu \mathrm{M}$ to $1.27 \mu \mathrm{M}$. While this resulted in an $\mathrm{IC}_{50}$ closer to that observed against $\mathrm{SpFabK}$, it still represents a roughly 19-fold increase and, therefore, also fails to fully explain the discrepancy. A final consideration is the possibility that the unique requirement of $C$. difficile for branched chain fatty acids may impact the substrate requirements of CdFabK, making a branched enoyl substrate more appropriate than Cro-CoA for compound screening, or even introducing the possibility of additional medicinal chemistry modifications to be made to the phenylimidazole compounds suited specifically to CdFabK.

Interestingly, a return to the benzothiazole scaffold of AG-205 resulted in the very recent discovery of a related compound, $\mathbf{K H - 7 0}$, that has proven to be the most potent $\mathrm{CdFabK}$ inhibitor to date. In comparison to the phenylimidazole analogues and AG-205, KH-70 possesses a shortened linker group and a sulfone moiety, either of which may help improve the observed potency (Figure 4-4). Further consideration of benzothiazole scaffolds is certainly warranted, and further $\mathbf{K H - 7 0}$ hit characterization and validation are ongoing. Without such studies, current rationale for the increased potency of KH-70 against $\mathrm{CdFabK}$ remains, to some extent, speculation. As such, necessary SAR studies are a primary goal for the future of this project.

\section{Summary}

With the continued use of broad-spectrum antibiotics, the increasingly diminishing spectrum of useful anti-CDI antibacterial agents, and the increased incidence of new pathogenic strains, the need for novel antibacterial agents targeting $C$. difficile continues to outpace their discovery. As demonstrated in these studies, a notably attractive target for antibacterial development resides in the bacterial FAS II pathway, which is structurally dissimilar from the mammalian fatty-acid synthesis (FAS I) pathway. ${ }^{204}$ FAS II also contains a number of dissimilar, independent enzymes and enzyme homologues collectively responsible for the synthesis of important bacterial fatty

acid precursors and, therefore, appears inherently well-suited for selective targeting. ${ }^{186,205}$ 


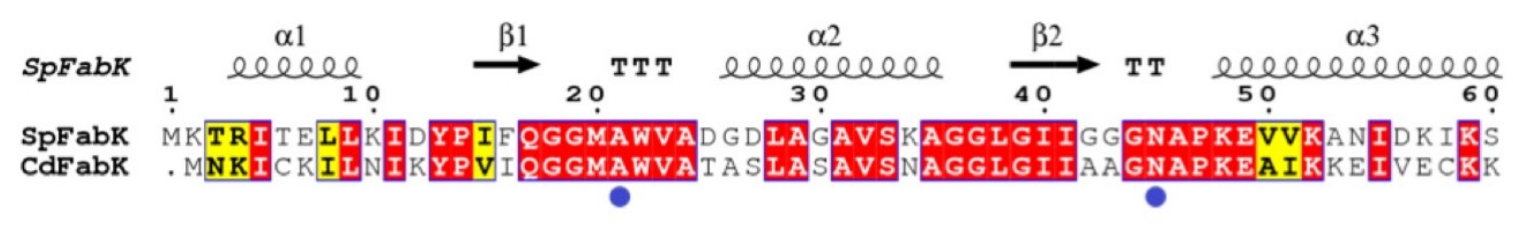

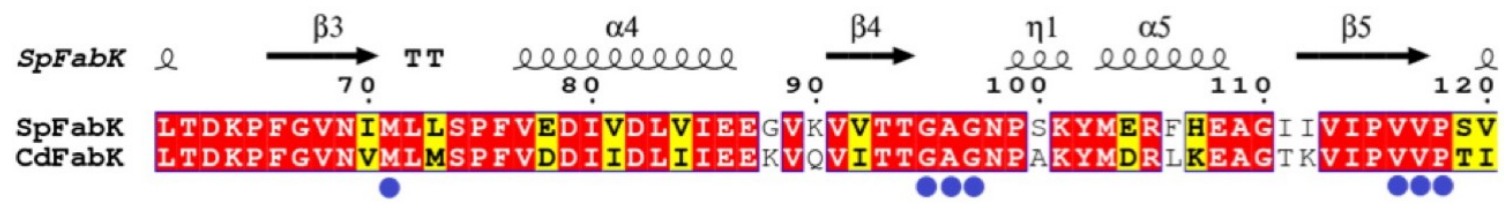

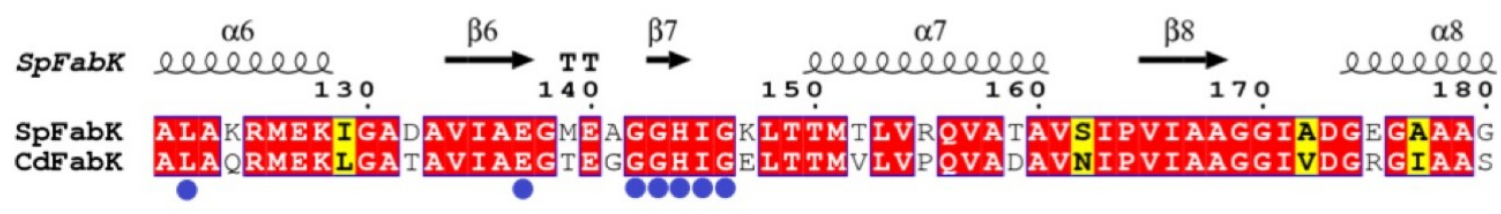

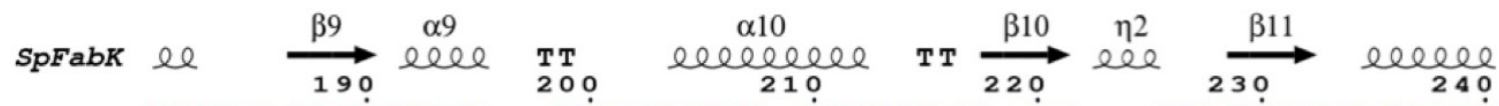
SPFabK FMLGAEAVQVGTRFVVAKES NAHP NYKEKILKARDIDT I ISAQHFGHAVRAIKNOLTRDF CdFabK EALGASAVQVGTREIC SEECSVHSNYKNLVLKAKDRDA IVTGRSTGHPVRTIKNKLSKEE

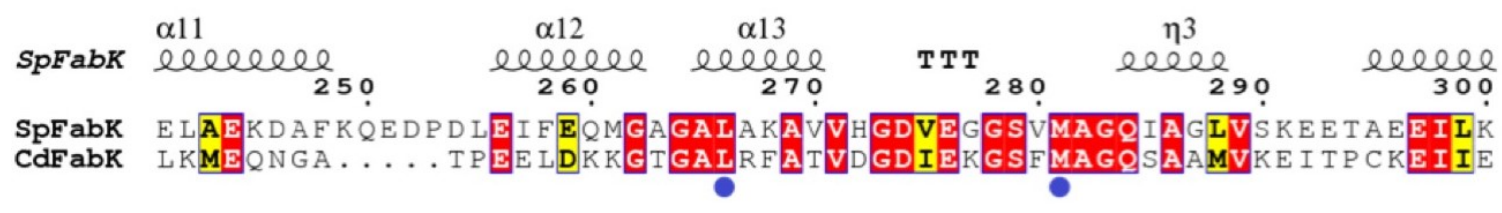

$\alpha 14$

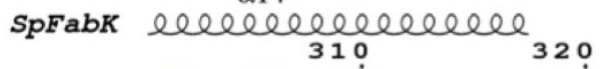

SPFabK DIYYGAAKK宁QEEASRWTGVVRND

CdFabK AMVNQAREIMPAIEL...........

\section{Figure 4-5. Sequence alignment comparing CdFabK and SpFabK.}

Identical residues are highlighted in red, similar residues are highlighted in yellow, and active site residues, within $4 \AA$ from phenylimidazole binding site, are indicated with a blue dot. ${ }^{119,122}$

Reprinted from final submission with permission from Jones, J. A.* et al. Small-molecule inhibition of the C. difficile FAS-II enzyme, FabK, results in selective activity. ACS Chem Biol, https://doi.org/10.1021/acschembio.9b00293. (2019. In Press). (*Co-first author.) Copyright (2019) American Chemical Society. ${ }^{139}$ 
Additionally, the pathway has now been well-validated as being essential in both Gramnegative bacteria and a number of Gram-positive bacteria, even in the presence of exogenous fatty acids.

Via direct comparison here of the phenylimidazole compounds' $\mathrm{IC}_{50}$ values against different purified enoyl-ACP reductase enzymes from different organisms, the selective druggability of CdFabK can be confirmed. Furthermore, analysis of in vitro MIC values of phenylimidazole compounds against various key gut microbes elsewhere in the literature has further illustrated this. ${ }^{67}$ As such, the selective inhibition of CdFabK represents a particularly promising pathway for future narrow-spectrum anti-difficile development. In vivo efficacy, toxicity, and dysbiosis studies in animal models of phenylimidazoles and derivatives will be indispensable for continued development and, therefore, are anticipated for future studies. Furthermore, as more information pertaining to the human microbiome continues to surface, an ongoing analysis of the distribution of current and novel FAS-II isozymes across the increasing number of known gut microbes will be of critical importance. Such analyses will guide an evolving understanding of the extent of antibacterial specificity and overall promise that targeting FabK truly offers. 


\section{CHAPTER 5. DISCUSSION AND CONCLUSION}

\section{General Overview}

Though the skillset required to address modern rational antibacterial drug development may be somewhat daunting at first glance, so is the rising threat of antibacterial resistance. It seems only fitting, then, that as the threat of resistance rises, so too must an equal measure of intellect and innovation to meet it. As even homologues of the same enzyme from different bacterial species may possess vastly different characteristics, there is no pre-determined roadmap for addressing the needs one may encounter when investigating novel antibacterial targets. Therefore, a wide and thorough understanding of the various methods and techniques needed for such endeavors is a necessity. Similarly, while even the best equipped laboratories likely do not possess every single piece of instrumentation available for drug discovery research, a wide knowledge base, experience, and familiarity with various methods and techniques offers the adaptability and expertise necessary to overcome even the most difficult projects. Indeed, understanding the advantages and disadvantages of different techniques, the overlap, and the subtle differences one may offer as opposed to another all combines to benefit the researcher.

While certainly not completely exhaustive, an effective combination of methods and techniques were used in this work to investigate narrow spectrum antibacterial drug targets. As can be observed, all of this work builds upon itself. From rational target identification, to target production and preliminary characterization, to compound screening and hit selection, to target and hit validation and characterization, and finally to lead development and optimization, the techniques employed in this work are representative of the necessary methodology required for a rational and early pre-clinical investigation of antibacterial drug targets. While the actual near-end stage of discovering and developing validated hits is indeed exciting and stands out from the rest of the work, it is the early, more tedious research that precedes those discoveries that serves as the actual foundation for later breakthroughs. For example, the arduous, repetitive science that led to the production of stabilized CdFabK enzyme and balanced assay conditions was an absolute prerequisite for the subsequent phenylimidazole CdFabK inhibitor validation and preliminary biochemical screening of virtual screening hits. A brief synopsis illustrating these very points is discussed below.

\section{Results and Discussion of Methods and Techniques Employed in This Work}

\section{Target Identification and Production}

Embarking on the investigation of arguably any novel and rational antibacterial drug target begins simply with a thorough analysis of the data at hand. Whether it is genomic data, microbiological data, or a basic literature search, the investigation of 
rational antibacterial targets requires just what the name implies, "rationalization." The work conducted herein marked the beginning of two different projects previously conceptualized by the principal investigator of the laboratory based off of rational navigation of literature and genomic data.

As seen in Chapter 2, at the time of inception, the bacterial topoisomerase I project was undertaken based on the data available at the time, which indicated that streptococci possessed the gene responsible for topoisomerase I, top $A$, and lacked the gene responsible for topoisomerase III, top $B$. After this work had commenced, however, protein annotations had arisen that introduced at least some level of doubt in both directions. As the lab in which this work was carried out does not conduct actual bacterial genetics research, a substantial amount of bioinformatic analysis was invested into ascertaining whether or not streptococci do in fact possess the top $B$ gene and, in turn, express topoisomerase III. This included genomic analyses, gene searches, protein searches, various BLAST searches, and various literature searches. At the time this work was written, this question still remained.

Significant preliminary work has been conducted in order to further characterize and validate bacterial topoisomerase I, including the development of a simplified and rapid protocol for the high-yield production of pure and active full-length topoisomerase I from the gram-positive model organism, S. mutans. Different affinity, fusion, and solubility tags were utilized, as well as different expression systems and purification modalities. The effectiveness of the protocol results in substantial target production that can later be used for further investigational studies and, therefore, still represents a valuable contribution. Whether or not streptococci produce topoisomerase III, the ability to effectively produce the bacterial topoisomerase I target in bulk amounts opens up a variety of studies that can be carried out, including but not limited to bacterial topoisomerase I enzymology, assay development, compound screening, and further structural studies. As several other notable pathogens express topoisomerase I as their sole type IA topoisomerase, high-yield production of target SmTopoI not only permits the continued investigation of the respective enzyme, but also affords the opportunity to further characterize and validate the target and potentially translate the findings to those pathogens where it is more certain bacterial topoisomerase I will represent a narrowspectrum antibacterial drug target. Furthermore, as topoisomerases are traditionally such effective chemotherapeutic targets, the option of pursuing bacterial topoisomerase I as a broad-spectrum antibacterial drug target also still exists.

With respect to the FabK project, effective collaborations ensued that answered genetic essentiality questions, highlighting the importance and benefit of such partnerships. ${ }^{67}$ Nonetheless, thorough bioinformatic analyses were also conducted with respect to the $f a b K$ gene and ongoing evaluations are being undertaken to survey the FAS II-related proteome of not only pathogenic bacteria, but also that of the ever-expanding bacteria that comprise the human microbiome. ${ }^{206-208}$ Such a continuous bioinformatic analysis has allowed for an ongoing and contemporaneous survey of the bacterial battle field, as it were, and in turn acts as a type of real-time reconnaissance of the effects FabK inhibition might have as pre-clinical research continues to progress. 
CdFabK enzyme target production evolved substantially throughout this work. Different affinity tag placements were combined with multiple purification techniques, along with systematic stability and activity studies in order to determine optimal salt, glycerol, and buffer and $\mathrm{pH}$ conditions. Ultimately, what was originally an inactive and later a relatively unstable enzyme, can now be produced and stored long-term as active enzyme.

\section{Preliminary Target Characterization}

Initial difficulty was met when attempting to produce and characterize SmTopoI. As stated earlier, the DNA-binding nature of the target introduced foundational difficulties with procuring stable enzyme. Stabilizing conditions were elucidated via implementation of the thermal shift assay, which indicated high salt and acidic stabilizing conditions for full-length SmTopoI. Simple target enzyme parameter analysis, coupled with in silico homology modeling and extensive literature searches led to rational shortened construct design that later resulted in highly diffracting SmTopoI_N65 protein crystals. $^{209}$

As mentioned above, CdFabK stability and activity was optimized via systematic salt, glycerol, and buffer and $\mathrm{pH}$ trials utilizing biochemical assays. This eventually permitted further target characterization that ultimately furnished effective assay conditions for inhibitor screening. Additionally, optimization of CdFabK stability under prolonged dilution and exposure to DMSO was of particular significance as many recent studies have underscored the importance of screening under extended enzyme-compound incubation times in order to discover slow-binding inhibitors. ${ }^{210-213}$

\section{Compound Screening and Hit Selection}

As previously discussed, an initial method for screening compounds is at times an intellectual one, first involving a thorough literature review in order to survey the chemical characteristics of any known potential inhibitors or chemical probes of interest. With respect to bacterial topoisomerase I, there is still no clinically marketed catalytic topoisomerase I inhibitor to date, nor is there a standard, specific bacterial topoisomerase I catalytic inhibitor reported in the literature for laboratory use. Regarding FabK, prototype phenylimidazole and benzothiazole compounds used in previous studies throughout the literature were found upon prior literature searches conducted by the principal investigator of the laboratory. ${ }^{163-166}$ These previously known SpFabK inhibitors served as the launching point for preliminary hit screening against $\mathrm{CdFabK}$ at $10 \mu \mathrm{M}$ as discussed earlier in this work. Moreover, these inhibitors and additional phenylimidazole analogues were similarly screened and hits were considered to be any compound that resulted in over $50 \% \mathrm{CdFabK}$ inhibition at the pre-determined $10 \mu \mathrm{M}$ concentration cutoff point. 


\section{Target and Hit Validation and Characterization}

From the attained SmTopoI_N65 crystals, the three-dimensional structure of SmTopoI_N65 was determined to $2.06 \AA$ resolution, and structural characterization was able to be conducted. This in turn resulted in a number of findings that significantly added to the overall structural understanding and characterization of SmTopoI. This includes overall similarities and differences between SmTopoI and topoisomerase I homologues from other bacteria. Of particular note, these structural studies revealed a relatively unique active site-adjacent loop that may be worth assessing in greater detail in the future.

A thorough assessment of those phenylimidazole analogues and benzothiazoles that met the $50 \% \mathrm{CdFabK}$ inhibition at $10 \mu \mathrm{M}$ hit identification threshold indeed led to further hit validation and $\mathrm{IC}_{50}$ knowledge that can be used in later lead optimization and structure-guided lead development. Furthermore, phenylimidazole inhibitor modality studies further validated the phenylimidazoles as CdFabK inhibitors and led to further inhibitor characterization. CdFabK enzymology studies led to apparent $K_{\mathrm{m}}$ determinations for the NADH cofactor and Cro-CoA enoyl substrate, which in turn led to optimal balanced assay conditions. This will allow for more effective compound screening in the future as it will increase the chances of discovering both competitive and uncompetitive inhibitors. Additional hit characterization was carried out via selectivity analysis, which confirmed selective inhibition of FabK over an alternative bacterial enoyl reductase, FabI.

\section{Lead Development}

With respect to $\mathrm{CdFabK}$ inhibitors, the phenylimidazole analogue $\mathbf{1 g}$ showed noteworthy merit as it displayed potency slightly lower yet very similar to the phenylimidazole prototype $\mathbf{1 b}$, but also showed increased solubility. Previous hit characterization allowed for the direct comparison of the $\mathrm{IC}_{50}$ values of the benzothiazole prototype, AG-205, against CdFabK and SpFabK. Such a comparison indeed had interesting and noteworthy implications. Namely, the medicinal chemistry modifications made to AG-205 to arrive at phenylimidazole $\mathbf{1 b}$ that led to substantially improved inhibitory potency against SpFabK (low micromolar to low nanomolar, respectively) does not appear to translate to increased potency against CdFabK. ${ }^{164,166}$ As such, a rational return to the original AG-205 benzothiazole scaffold was undertaken for an SAR reset to be conducted more thoroughly in the future for effective lead development. In the meantime, the alternative benzothiazole compound KH-70 recently exhibited the highest observed inhibitory potency against $\mathrm{CdFabK}$ at the time of this work, suggesting at least some degree of merit in this lead development strategy. 


\section{Future Directions}

The research presented here showcases the investigation of two very different narrow spectrum targets in antibacterial drug discovery. Both projects presented unique obstacles and successes and, therefore, required the implementation of various subjective techniques and methods. While various aspects of each project progressed to different stages, work remains regarding both projects.

\section{Bacterial Topoisomerase I Project}

\section{Genomic analysis of streptococci}

The lingering uncertainty regarding whether or not streptococci possess the top $B$ gene, and therefore whether or not streptococci indeed fit the original narrow spectrum hypothesis, represents a quandary that must be resolved. As such, determining whether or not streptococci possesses the top $B$ gene and, therefore, whether or not they express topoisomerase III should be a top priority for future work. Further bioinformatic analyses would offer more information, but certainty is likely to only be found in benchtop bacterial genetic work. As such work is outside the scope of the principal investigator's laboratory as it equipped at this time, a gifted collaborator with expertise in bacterial genetics and, possibly, a research emphasis on streptococci would be of significant worth to the future progress of this project.

\section{Full-length SmTopoI crystal structure}

Useful insights were indeed gleaned from the determination of the threedimensional structure of the shortened $65-\mathrm{kDa}$ amino terminal fragment of SmTopoI, SmTopoI_N65. However, as SmTopoI_N65 lacks the entire carboxy terminal domain, a full-length structure of SmTopoI would contribute substantial structural information that could potentially in turn be leveraged into effective structure-guided drug development efforts. As it was already exhaustively determined to be unlikely, or at the very least exceptionally difficult, to obtain full-length apo SmTopoI crystals, it is likely that cocrystallography trials involving short oligonucleotide substrates with or without additive compounds would be of immediate benefit with respect to this particular aim. As SAR studies are an eventual long-term goal, this is a logical next step for this project.

\section{SmTopoI mutational studies}

Knowledge gleaned from the determination of the SmTopoI_N65 crystal structure includes the relatively unique nine amino acid loop extension immediately adjacent to the active site. As this feature has not been discussed or elucidated any further in the literature, a deeper understanding may be warranted. In order to probe any significance of the loop, mutational studies could be undertaken to delete the loop and compare mutant SmTopoI to wild-type SmTopoI. Any changes in enzyme kinetics or nucleotide binding 
specificity would be a significant discovery and, whether any notable change occurred or not, would further the understanding of the loop extension. Additionally, it may help ascertain whether or not the loop would be worth targeting or engaging for any future rational antibacterial drug discovery efforts.

\section{High throughput topoisomerase I assay development}

Currently, the only well-validated assay for measuring the activity of bacterial topoisomerase I is an agarose gel-based DNA relaxation assay. This technique, while effective, is extremely low-throughput and is not immediately digitized for consistent comparisons. As such, an immediate need for successful antibacterial drug discovery efforts directed at topoisomerase I includes the development of a high throughput assay. This would allow for effective and efficient screening of large compound libraries as well as efficient enzymatic studies. As bacterial topoisomerase I acts independently of ATP and instead relies on the torsional strain of negatively supercoiled DNA, the only measurable entity for assaying is the actual extent of DNA supercoiling itself, which presents an inherently difficult task. Nonetheless, such a development would be a revolutionary advancement with respect to targeting bacterial topoisomerase I and is highly needed.

\section{CdFabK Project}

\section{Crystallography}

Though diffracting CdFabK protein crystals have been acquired, the optimal crystals thus far have only diffracted to roughly $3.5 \AA$. In order to serve any real utility in structure-guided drug discovery efforts, high quality crystals that diffract near $2.0 \AA$ or better are necessary to analyze atomic level interactions and peptide sidechains. To this end, numerous crystallography methods have been attempted up to this point to no avail. Countless alternative crystallization methods remain to be tried, though, ranging from immediately accessible techniques to those that are more arduous and time consuming. Methods that could be attempted immediately include but are not limited to cocrystallizing different additive screens, co-crystallization trials with newly found inhibitor like KH-70, producing the target enzyme in alternative buffers, increased purification steps to remove any remaining contaminant proteins that may be interfering with crystal packing and quality, and various digestion methods. ${ }^{214}$

More time consuming and taxing methods should also be explored, include various target enzyme mutational or deletional studies for the purpose of construct optimization, including modifying solvent exposed hydrophobic or flexible peptide regions that may result in non-specific aggregation and compromise ordered crystal lattice formation and organization. ${ }^{215,216}$ To this end, the determination of the CdFabK structure from the current best diffracting crystals may only result in the discernment of overall structure, but it may also afford insight into the crystallographic unit cell and crystal packing modalities, which may help drive the rationalization strategy behind any 
future mutational studies. While all of these options are worthwhile endeavors, for the mere sake of expediting the process, co-crystallography with newly discovered potent CdFabK inhibitors would likely be an ideal place to start the next phase of a crystallization campaign.

\section{Further hit validation and characterization}

As the benzothiazole analogue $\mathrm{KH}-70$ was an extremely recent hit, further hit validation and characterization need to be conducted. Similarly, additional SAR studies should be undertaken, focusing on benzothiazole analogue SAR by synthetic medicinal chemistry, or SAR by commerce (see below).

\section{Compound screening}

The optimized CdFabK assay conditions should be leveraged as practicably and aggressively as possible for the discovery of novel CdFabK inhibitors. Small in-house libraries can be immediately screened. Likewise, virtual screening campaigns have already commenced by others in the laboratory, and high-priority in silico hits can be screened and verified in vitro and further validated. The utility of discovering inhibitors with both novel and similar scaffolds would be substantial. Novel scaffolds would introduce a new SAR opportunity and help further characterize the CdFabK target, and analogues of known inhibitor scaffolds would further elucidate current SAR studies. Related to known scaffolds, analogues of current inhibitors could be searched for online as a time- and cost-effective means of conducting SAR by commerce, effectively furthering iterative lead development and SAR expansion on the phenylimidazole and benzothiazole compounds. ${ }^{217,218}$

\section{Orthogonal high throughput assay development}

The current CdFabK assay methods involve monitoring the fluorescence or absorbance of NADH during the enzymatic reaction. As many small molecules found in various compound screening libraries are in fact fluorescent or colored, substantial amounts of false positives can arise when using fluorescent- or absorbance-based assays. ${ }^{219}$ An orthogonal assay capable of counter-screening and further validating any potential hits discovered upon initial fluorescence- or absorbance-based assays (or vice versa) would be of substantial value. Any such assay should possess a number of ideal characteristics. First, it should be orthogonal to fluorescence- and absorbance-based assays, as discussed, such as a chemiluminescent assay. It should also be plate-based in order to accommodate high throughput implementation. Additionally, the ability to be run in low-volume would be beneficial as it would decrease resource consumption and assist in the high throughput requirement. Low cost would be ideal, but not a necessity as high throughput screens are generally accepted as rather costly endeavors. And lastly, the assay should be capable of producing a significantly high screening window coefficient, or Z' factor, under moderate controls in order to reliably report any discovered hits at the predetermined thresholds. ${ }^{220,221}$ 


\section{Conclusions}

Despite the recent increase in the number of novel antibacterial agents in the pipeline, there is still a noticeably small number of agents that possess novel mechanisms of action, and an even more noticeable absence of small-molecule compounds directed at novel targets. In fact, the only antibacterial agent currently in the pipeline that is directed at a novel target is the anti-pseudomonal synthetic beta hairpin peptidomimetic known as murepavadin (POL7080, Polyphor, Ltd.) currently in late stage (Phase III) development. ${ }^{6,38}$ While absolutely a welcome contribution to the fight against antimicrobial resistance, murepavadin does not meet the criteria of being a "small molecule," and instead falls into the category of novel biologic agents increasing in popularity across most areas of drug discovery. ${ }^{222}$ Again, while any novel antibacterial agent is desirable, including any biologic, the desirable benefits of small-molecule antibacterial agents should not be overlooked. This is especially the case when considering antibacterials. Due to their inherent purpose, antibacterial agents in particular need to exhibit significant stability, cost-effectiveness, and ease of widespread administration. Rationale for this is due in part to the necessity for antibacterial stockpiling, dispersal in potentially harsh climates where many major infectious diseases are focused, and the need for rapid response to outbreaks or bioterror events. In general, biologics are more expensive than small-molecule drugs, less stable, more likely to cause adverse immune responses, and more difficult to administer. ${ }^{223}$ These points, on top of the aforementioned fact that there are currently no novel small-molecule antibacterial agents with novel targets in the current antibacterial drug discovery pipeline, highlight the continuing and apparent need that still exists to fill this void.

Obvious merit exists for the development of narrow-spectrum antibacterial agents, and calls for such are only gaining momentum. ${ }^{2,54}$ As discussed throughout this work, significant advantages are associated with narrow spectrum antibacterial agents - mainly the counter to the disadvantages seen with broad-spectrum antibacterial agents. In general, the major benefits of narrow-spectrum agents include an attenuation of microbial selection for wide-spread multidrug resistance pertaining not only to the pathogens necessitating pharmacotherapy, but also commensal bacteria capable of harboring and spreading resistance mechanisms, as well as the related mitigation of collateral damage to the human microbiome. Though narrow-spectrum antibacterial drug discovery appears to hold great potential value, it is not without significant price. As showcased in this work, a substantial amount of rationalization and investment - intellectual or otherwise - is required in the pursuit of narrow-spectrum targets in antibacterial drug discovery. This includes target identification and production, preliminary target characterization, compound screening and hit selection, further target and hit validation and characterization, iterative lead development, followed by continued narrow-spectrum activity and dysbiosis analyses on top of all of that. While indeed difficult, different research groups are coming up with innovative modalities of identifying and pursuing narrow-spectrum targets. Modern methods include targeting enzymes that are unique and essential to specific pathogens of interest (as seen in this work), pursuing bacteriocins and synthetic peptides that only target particular pathogens and, interestingly, a return to the 
whole-cell phenotypic screening modalities reminiscent of those used throughout the golden era of antibacterial drug discovery. ${ }^{2,38,54,67}$

After surveying the global issue of antibacterial resistance and the concerted efforts being undertaken to combat it, it is clear that a multi-faceted approach is required to effectively respond to this growing threat. As such, it would be inaccurate to argue that the only answer to the issues currently presented lies in narrow-spectrum antibacterials. Instead, it represents a single component of a plurality of different approaches needed. As long as infectious disease diagnostics are not instantaneous, broad-spectrum antibacterial agents will always be necessary for empiric therapy. In an ideal situation, after an accurate diagnosis is made, antibacterial de-escalation could be implemented and a patient could be transitioned to a narrow-spectrum antibacterial therapy, thereby effectively treating an infection and mitigating both the damage done to the patient's microbiome, and mitigating the promotion of antibacterial drug resistance. For this ideal situation to occur, however, substantial progress still needs to be made toward the development of narrow-spectrum antibacterial agents, and such progress begins with earnest investment in the investigation of narrow-spectrum targets in antibacterial drug discovery. This work highlights such investment and, in turn, progress with respect to the investigation of both bacterial topoisomerase I and bacterial enoyl-acyl carrier protein (ACP) reductase II, or FabK, as narrow-spectrum targets in antibacterial drug discovery. 


\section{LIST OF REFERENCES}

1 Jones, J. A., Virga, K. G., Gumina, G. \& Hevener, K. E. Recent Advances in the Rational Design and Optimization of Antibacterial Agents. Medchemcomm 7, 1694-1715, doi:10.1039/C6MD00232C (2016).

2 Brown, E. D. \& Wright, G. D. Antibacterial drug discovery in the resistance era. Nature 529, 336-343, doi:10.1038/nature17042 (2016).

3 Cooper, M. A. \& Shlaes, D. Fix the antibiotics pipeline. Nature 472, 32, doi:10.1038/472032a (2011).

4 Payne, D. J., Gwynn, M. N., Holmes, D. J. \& Pompliano, D. L. Drugs for bad bugs: confronting the challenges of antibacterial discovery. Nat Rev Drug Discov 6, 29-40, doi:10.1038/nrd2201 (2007).

5 IACG. No Time to Wait: Securing the future from drug-resistant infections. World Health Organization (WHO) Report. (2019).

6 Talbot, G. H. et al. The Infectious Diseases Society of America's 10 x '20 Initiative (Ten New Systemic Antibacterial Agents FDA-approved by 2020): Is 20 x '20 a Possibility? Clin Infect Dis, doi:10.1093/cid/ciz089 (2019).

7 Boucher, H. W. et al. Bad bugs, no drugs: no ESKAPE! An update from the Infectious Diseases Society of America. Clin Infect Dis 48, 1-12, doi:10.1086/595011 (2009).

8 Infectious Diseases Society of, A. The $10 \times$ '20 Initiative: pursuing a global commitment to develop 10 new antibacterial drugs by 2020. Clin Infect Dis $\mathbf{5 0}$, 1081-1083, doi:10.1086/652237 (2010).

9 Boucher, H. W. et al. $10 \times$ ' 20 Progress--development of new drugs active against gram-negative bacilli: an update from the Infectious Diseases Society of America. Clin Infect Dis 56, 1685-1694, doi:10.1093/cid/cit152 (2013).

10 CDC. Antibiotic resistance threats in the United States, 2013. Centers for Disease Control and Prevention (CDC). (2013).

11 Pawlowski, A. C. et al. A diverse intrinsic antibiotic resistome from a cave bacterium. Nat Commun 7, 13803, doi:10.1038/ncomms13803 (2016).

12 Lewis, K. Platforms for antibiotic discovery. Nat Rev Drug Discov 12, 371-387, doi:10.1038/nrd3975 (2013).

13 Ling, L. L. et al. A new antibiotic kills pathogens without detectable resistance. Nature 517, 455-459, doi:10.1038/nature14098 (2015).

14 Moellering, R. C. Linezolid: the first oxazolidinone antimicrobial. Annals of internal medicine 138, 135-142 (2003).

15 Stryjewski, M. E. \& Corey, G. R. Methicillin-resistant Staphylococcus aureus: an evolving pathogen. Clin Infect Dis 58 Suppl 1, S10-19, doi:10.1093/cid/cit613 (2014).

16 Locke, J. B. et al. Linezolid-resistant Staphylococcus aureus strain 1128105, the first known clinical isolate possessing the cfr multidrug resistance gene. Antimicrob Agents Chemother 58, 6592-6598, doi:10.1128/AAC.03493-14 (2014). 
17 Morales, G. et al. Resistance to linezolid is mediated by the cfr gene in the first report of an outbreak of linezolid-resistant Staphylococcus aureus. Clin Infect Dis 50, 821-825, doi:10.1086/650574 (2010).

18 Redgrave, L. S., Sutton, S. B., Webber, M. A. \& Piddock, L. J. Fluoroquinolone resistance: mechanisms, impact on bacteria, and role in evolutionary success. Trends Microbiol 22, 438-445, doi:10.1016/j.tim.2014.04.007 (2014).

19 Miller, A. A. et al. Discovery and characterization of QPT-1, the progenitor of a new class of bacterial topoisomerase inhibitors. Antimicrob Agents Chemother 52, 2806-2812, doi:10.1128/AAC.00247-08 (2008).

20 Sum, P. E. \& Petersen, P. Synthesis and structure-activity relationship of novel glycylcycline derivatives leading to the discovery of GAR-936. Bioorg Med Chem Lett 9, 1459-1462 (1999).

21 Bergeron, J. et al. Glycylcyclines bind to the high-affinity tetracycline ribosomal binding site and evade Tet(M)- and Tet(O)-mediated ribosomal protection. Antimicrob Agents Chemother 40, 2226-2228 (1996).

22 Petersen, P. J., Jacobus, N. V., Weiss, W. J., Sum, P. E. \& Testa, R. T. In vitro and in vivo antibacterial activities of a novel glycylcycline, the 9-tbutylglycylamido derivative of minocycline (GAR-936). Antimicrobial agents and chemotherapy 43, 738-744 (1999).

23 Sivapalasingam, S. \& Steigbigel, N. H. in Mandell, Douglas, and Bennett's Principles and Practice of Infectious Diseases Vol. 1 (eds J. E. Bennett, R. Dolin, \& M. J. Blaser) Ch. 29, 358-376 (Saunders - Elsevier, Inc., 2014).

24 Zhanel, G. G. et al. Comparison of the next-generation aminoglycoside plazomicin to gentamicin, tobramycin and amikacin. Expert Rev Anti Infect Ther 10, 459-473, doi:10.1586/eri.12.25 (2012).

25 Martins, A. F. et al. Antimicrobial activity of plazomicin against Enterobacteriaceae-producing carbapenemases from 50 Brazilian medical centers. Diagn Microbiol Infect Dis 90, 228-232, doi:10.1016/j.diagmicrobio.2017.11.004 (2018).

26 Oefner, C. et al. Increased hydrophobic interactions of iclaprim with Staphylococcus aureus dihydrofolate reductase are responsible for the increase in affinity and antibacterial activity. $J$ Antimicrob Chemother 63, 687-698, doi:10.1093/jac/dkp024 (2009).

27 Schneider, P., Hawser, S. \& Islam, K. Iclaprim, a novel diaminopyrimidine with potent activity on trimethoprim sensitive and resistant bacteria. Bioorg Med Chem Lett 13, 4217-4221 (2003).

28 Sincak, C. A. \& Schmidt, J. M. Iclaprim, a novel diaminopyrimidine for the treatment of resistant gram-positive infections. Ann Pharmacother 43, 1107-1114, doi:10.1345/aph.1L167 (2009).

29 Schiebel, J. et al. Rational design of broad spectrum antibacterial activity based on a clinically relevant enoyl-acyl carrier protein (ACP) reductase inhibitor. $J$ Biol Chem 289, 15987-16005, doi:10.1074/jbc.M113.532804 (2014).

30 Gerusz, V. et al. From triclosan toward the clinic: discovery of nonbiocidal, potent FabI inhibitors for the treatment of resistant bacteria. J Med Chem 55, 9914-9928, doi:10.1021/jm301113w (2012). 
31 Karlowsky, J. A., Kaplan, N., Hafkin, B., Hoban, D. J. \& Zhanel, G. G. AFN1252, a FabI inhibitor, demonstrates a Staphylococcus-specific spectrum of activity. Antimicrob Agents Chemother 53, 3544-3548, doi:10.1128/AAC.0040009 (2009).

32 Karlowsky, J. A. et al. In vitro activity of API-1252, a novel FabI inhibitor, against clinical isolates of Staphylococcus aureus and Staphylococcus epidermidis. Antimicrob Agents Chemother 51, 1580-1581, doi:10.1128/AAC.01254-06 (2007).

33 Kaplan, N. et al. Mode of action, in vitro activity, and in vivo efficacy of AFN1252, a selective antistaphylococcal FabI inhibitor. Antimicrob Agents Chemother 56, 5865-5874, doi:10.1128/AAC.01411-12 (2012).

34 Kahne, D., Leimkuhler, C., Lu, W. \& Walsh, C. Glycopeptide and lipoglycopeptide antibiotics. Chem Rev 105, 425-448, doi:10.1021/cr030103a (2005).

35 Birnbaum, J., Kahan, F. M., Kropp, H. \& MacDonald, J. S. Carbapenems, a new class of beta-lactam antibiotics. Discovery and development of imipenem/cilastatin. Am J Med 78, 3-21 (1985).

36 Lutgring, J. D. Carbapenem-resistant Enterobacteriaceae: An emerging bacterial threat. Semin Diagn Pathol, doi:10.1053/j.semdp.2019.04.011 (2019).

37 Sykes, R. B., Wells, J. S., Parker, W. L., Koster, W. H. \& Cimarusti, C. M. Aztreonam: discovery and development of the monobactams. $N J$ Med Spec No, 8-15 (1986).

38 Robinson, J. A. Folded Synthetic Peptides and Other Molecules Targeting Outer Membrane Protein Complexes in Gram-Negative Bacteria. Front Chem 7, 45, doi:10.3389/fchem.2019.00045 (2019).

39 Chen, L., Todd, R., Kiehlbauch, J., Walters, M. \& Kallen, A. Notes from the Field: Pan-Resistant New Delhi Metallo-Beta-Lactamase-Producing Klebsiella pneumoniae - Washoe County, Nevada, 2016. MMWR Morb Mortal Wkly Rep 66, 33, doi:10.15585/mmwr.mm6601a7 (2017).

40 Thomas, S. E. et al. Structural Biology and the Design of New Therapeutics: From HIV and Cancer to Mycobacterial Infections: A Paper Dedicated to John Kendrew. J Mol Biol 429, 2677-2693, doi:10.1016/j.jmb.2017.06.014 (2017).

41 Smith, D. L., Deng, Y. \& Zhang, Z. Probing the non-covalent structure of proteins by amide hydrogen exchange and mass spectrometry. J Mass Spectrom 32, 135146, doi:10.1002/(SICI)1096-9888(199702)32:2<135::AID-JMS486>3.0.CO;2-M (1997).

42 Huynh, K. \& Partch, C. L. Analysis of protein stability and ligand interactions by thermal shift assay. Curr Protoc Protein Sci 79, 2829 21-14, doi:10.1002/0471140864.ps2809s79 (2015).

43 Patching, S. G. Surface plasmon resonance spectroscopy for characterisation of membrane protein-ligand interactions and its potential for drug discovery. Biochimica et biophysica acta 1838, 43-55, doi:10.1016/j.bbamem.2013.04.028 (2014).

44 Damian, L. Isothermal titration calorimetry for studying protein-ligand interactions. Methods Mol Biol 1008, 103-118, doi:10.1007/978-1-62703-398-5_4 (2013). 
45 Hughes, J. P., Rees, S., Kalindjian, S. B. \& Philpott, K. L. Principles of early drug discovery. Br J Pharmacol 162, 1239-1249, doi:10.1111/j.14765381.2010.01127.x (2011).

46 Hirano, Y., Kimura, S. \& Tamada, T. High-resolution crystal structures of the solubilized domain of porcine cytochrome b5. Acta Crystallogr D Biol Crystallogr 71, 1572-1581, doi:10.1107/S1399004715009438 (2015).

47 Murata, K. \& Wolf, M. Cryo-electron microscopy for structural analysis of dynamic biological macromolecules. Biochim Biophys Acta Gen Subj 1862, 324334, doi:10.1016/j.bbagen.2017.07.020 (2018).

48 Scapin, G., Potter, C. S. \& Carragher, B. Cryo-EM for Small Molecules Discovery, Design, Understanding, and Application. Cell Chem Biol 25, 13181325, doi:10.1016/j.chembiol.2018.07.006 (2018).

49 Merk, A. et al. Breaking Cryo-EM Resolution Barriers to Facilitate Drug Discovery. Cell 165, 1698-1707, doi:10.1016/j.cell.2016.05.040 (2016).

50 Wishart, D. NMR spectroscopy and protein structure determination: applications to drug discovery and development. Curr Pharm Biotechnol 6, 105-120 (2005).

51 Frueh, D. P., Goodrich, A. C., Mishra, S. H. \& Nichols, S. R. NMR methods for structural studies of large monomeric and multimeric proteins. Curr Opin Struct Biol 23, 734-739, doi:10.1016/j.sbi.2013.06.016 (2013).

52 Sugiki, T., Kobayashi, N. \& Fujiwara, T. Modern Technologies of Solution Nuclear Magnetic Resonance Spectroscopy for Three-dimensional Structure Determination of Proteins Open Avenues for Life Scientists. Comput Struct Biotechnol J 15, 328-339, doi:10.1016/j.csbj.2017.04.001 (2017).

53 Zang, P., Gong, A., Zhang, P. \& Yu, J. Targeting druggable enzymome by exploiting natural medicines: An in silico-in vitro integrated approach to combating multidrug resistance in bacterial infection. Pharm Biol 54, 604-618, doi:10.3109/13880209.2015.1068338 (2016).

54 Melander, R. J., Zurawski, D. V. \& Melander, C. Narrow-Spectrum Antibacterial Agents. Medchemcomm 9, 12-21, doi:10.1039/C7MD00528H (2018).

55 Pommier, Y. Drugging topoisomerases: lessons and challenges. ACS Chem Biol 8, 82-95, doi:10.1021/cb300648v (2013).

56 Tse-Dinh, Y. C. Bacterial topoisomerase I as a target for discovery of antibacterial compounds. Nucleic Acids Res. 37, 731-737, doi:10.1093/nar/gkn936 (2009).

57 Stockum, A., Lloyd, R. G. \& Rudolph, C. J. On the viability of Escherichia coli cells lacking DNA topoisomerase I. BMC Microbiol 12, 26, doi:10.1186/14712180-12-26 (2012).

58 Liu, I. F., Sutherland, J. H., Cheng, B. \& Tse-Dinh, Y. C. Topoisomerase I function during Escherichia coli response to antibiotics and stress enhances cell killing from stabilization of its cleavage complex. J. Antimicrob. Chemother. 66, 1518-1524, doi:10.1093/jac/dkr150 (2011).

59 Ahmed, W., Menon, S., Godbole, A. A., Karthik, P. V. \& Nagaraja, V. Conditional silencing of topoisomerase I gene of Mycobacterium tuberculosis validates its essentiality for cell survival. FEMS Microbiol Lett 353, 116-123, doi:10.1111/1574-6968.12412 (2014). 
60 Ajdic, D. et al. Genome sequence of Streptococcus mutans UA159, a cariogenic dental pathogen. Proc. Natl. Acad. Sci. U. S. A. 99, 14434-14439, doi:10.1073/pnas.172501299 (2002).

61 Maruyama, F. et al. Comparative genomic analyses of Streptococcus mutans provide insights into chromosomal shuffling and species-specific content. BMC Genomics 10, 358, doi:10.1186/1471-2164-10-358 (2009).

62 Aikawa, C. et al. Complete genome sequence of the serotype k Streptococcus mutans strain LJ23. J Bacteriol 194, 2754-2755, doi:10.1128/JB.00350-12 (2012).

$63 \mathrm{Xu}, \mathrm{P}$. et al. Genome-wide essential gene identification in Streptococcus sanguinis. Sci. Rep. 1, 125, doi:10.1038/srep00125 (2011).

64 Molzen, T. E. et al. Genome-wide identification of Streptococcus pneumoniae genes essential for bacterial replication during experimental meningitis. Infect Immun 79, 288-297, doi:10.1128/IAI.00631-10 (2011).

65 Song, J. H. \& Ko, K. S. Detection of essential genes in Streptococcus pneumoniae using bioinformatics and allelic replacement mutagenesis. Methods Mol Biol 416, 401-408, doi:10.1007/978-1-59745-321-9_28 (2008).

66 Song, J. H. et al. Identification of essential genes in Streptococcus pneumoniae by allelic replacement mutagenesis. Mol. Cells 19, 365-374 (2005).

67 Marreddy, R. K. R. et al. The Fatty Acid Synthesis Protein Enoyl-ACP Reductase II (FabK) is a Target for Narrow-Spectrum Antibacterials for Clostridium difficile Infection. ACS Infect Dis 5, 208-217, doi:10.1021/acsinfecdis.8b00205 (2019).

68 Heath, R. J., Su, N., Murphy, C. K. \& Rock, C. O. The enoyl-[acyl-carrierprotein] reductases FabI and FabL from Bacillus subtilis. J Biol Chem 275, 40128-40133, doi:10.1074/jbc.M005611200 (2000).

69 Marrakchi, H. et al. Characterization of Streptococcus pneumoniae enoyl-(acylcarrier protein) reductase (FabK). Biochem J 370, 1055-1062, doi:10.1042/BJ20021699 (2003).

70 Massengo-Tiasse, R. P. \& Cronan, J. E. Vibrio cholerae FabV defines a new class of enoyl-acyl carrier protein reductase. J Biol Chem 283, 1308-1316, doi:10.1074/jbc.M708171200 (2008).

71 Parsons, J. B. \& Rock, C. O. Is bacterial fatty acid synthesis a valid target for antibacterial drug discovery? Current opinion in microbiology 14, 544-549, doi:10.1016/j.mib.2011.07.029 (2011).

72 Parsons, J. B. \& Rock, C. O. Bacterial lipids: metabolism and membrane homeostasis. Progress in lipid research 52, 249-276, doi:10.1016/j.plipres.2013.02.002 (2013).

73 Jones, J. A., Price, E., Miller, D. \& Hevener, K. E. A simplified protocol for highyield expression and purification of bacterial topoisomerase I. Protein Expr Purif 124, 32-40, doi:10.1016/j.pep.2016.04.010 (2016).

74 Jones, J. A. \& Hevener, K. E. Crystal structure of the 65-kilodalton aminoterminal fragment of DNA topoisomerase I from the gram-positive model organism Streptococcus mutans. Biochem Biophys Res Commun, doi:10.1016/j.bbrc.2019.06.034 (2019, In Press).

75 Champoux, J. J. DNA topoisomerases: structure, function, and mechanism. Annu Rev Biochem 70, 369-413, doi:10.1146/annurev.biochem.70.1.369 (2001). 
76 Slesarev, A. I. et al. DNA topoisomerase V is a relative of eukaryotic topoisomerase I from a hyperthermophilic prokaryote. Nature 364, 735-737, doi:10.1038/364735a0 (1993).

77 Wendorff, T. J. \& Berger, J. M. Topoisomerase VI senses and exploits both DNA crossings and bends to facilitate strand passage. Elife 7, doi:10.7554/eLife.31724 (2018).

78 Forterre, P., Gribaldo, S., Gadelle, D. \& Serre, M. C. Origin and evolution of DNA topoisomerases. Biochimie 89, 427-446, doi:10.1016/j.biochi.2006.12.009 (2007).

79 Wang, J. C. DNA topoisomerases: why so many? J Biol Chem 266, 6659-6662 (1991).

80 Mayer, C. \& Janin, Y. L. Non-quinolone inhibitors of bacterial type IIA topoisomerases: a feat of bioisosterism. Chem Rev 114, 2313-2342, doi:10.1021/cr4003984 (2014).

81 Nimesh, H. et al. Synthesis and biological evaluation of novel bisbenzimidazoles as Escherichia coli topoisomerase IA inhibitors and potential antibacterial agents. J. Med. Chem. 57, 5238-5257, doi:10.1021/jm5003028 (2014).

82 Sng, J. H. et al. Molecular cloning and characterization of the human topoisomerase IIalpha and IIbeta genes: evidence for isoform evolution through gene duplication. Biochim Biophys Acta 1444, 395-406 (1999).

83 Stupina, V. A. \& Wang, J. C. Viability of Escherichia coli topA mutants lacking DNA topoisomerase I. J Biol Chem 280, 355-360, doi:10.1074/jbc.M411924200 (2005).

84 Clark, K., Karsch-Mizrachi, I., Lipman, D. J., Ostell, J. \& Sayers, E. W. GenBank. Nucleic Acids Res 44, D67-72, doi:10.1093/nar/gkv1276 (2016).

85 Altschul, S. F., Gish, W., Miller, W., Myers, E. W. \& Lipman, D. J. Basic local alignment search tool. $J$ Mol Biol 215, 403-410, doi:10.1016/S00222836(05)80360-2 (1990).

86 Brenciani, A. et al. Genetic determinants and elements associated with antibiotic resistance in viridans group streptococci. J. Antimicrob. Chemother. 69, 11971204, doi:10.1093/jac/dkt495 (2014).

87 Doern, C. D. \& Burnham, C. A. It's not easy being green: the viridans group streptococci, with a focus on pediatric clinical manifestations. J. Clin. Microbiol. 48, 3829-3835, doi:10.1128/JCM.01563-10 (2010).

88 Shelburne, S. A. et al. Streptococcus mitis strains causing severe clinical disease in cancer patients. Emerg. Infect. Dis. 20, 762-771, doi:10.3201/eid2005.130953 (2014).

89 Sullivan, R. et al. Clinical efficacy of a specifically targeted antimicrobial peptide mouth rinse: targeted elimination of Streptococcus mutans and prevention of demineralization. Caries Res. 45, 415-428, doi:10.1159/000330510 (2011).

90 Stoopler, E. T. \& Sollecito, T. P. Oral mucosal diseases: evaluation and management. Med. Clin. North Am. 98, 1323-1352, doi:10.1016/j.mcna.2014.08.006 (2014).

91 Pfaller, M. A. \& Diekema, D. J. Epidemiology of invasive candidiasis: a persistent public health problem. Clin. Microbiol. Rev. 20, 133-163, doi:10.1128/CMR.00029-06 (2007). 
92 Rineh, A., Kelso, M. J., Vatansever, F., Tegos, G. P. \& Hamblin, M. R. Clostridium difficile infection: molecular pathogenesis and novel therapeutics. Expert Rev. Anti Infect. Ther. 12, 131-150, doi:10.1586/14787210.2014.866515 (2014).

93 Garcia, M. T. et al. New alkaloid antibiotics that target the DNA topoisomerase I of Streptococcus pneumoniae. J Biol Chem 286, 6402-6413, doi:10.1074/jbc.M110.148148 (2011).

94 Duan, F. et al. Haloemodin as novel antibacterial agent inhibiting DNA gyrase and bacterial topoisomerase I. J Med Chem 57, 3707-3714, doi:10.1021/jm401685f (2014).

95 Tan, K., Cao, N., Cheng, B., Joachimiak, A. \& Tse-Dinh, Y. C. Insights from the Structure of Mycobacterium tuberculosis Topoisomerase I with a Novel Protein Fold. J Mol Biol 428, 182-193, doi:10.1016/j.jmb.2015.11.024 (2016).

96 Tan, K. et al. Structural basis for suppression of hypernegative DNA supercoiling by E. coli topoisomerase I. Nucleic Acids Res 43, 11031-11046, doi:10.1093/nar/gkv1073 (2015).

97 Liu, I. F., Sutherland, J. H., Cheng, B. \& Tse-Dinh, Y. C. Topoisomerase I function during Escherichia coli response to antibiotics and stress enhances cell killing from stabilization of its cleavage complex. J Antimicrob Chemother 66, 1518-1524, doi:10.1093/jac/dkr150 (2011).

98 Burgess, R. R. Use of polyethyleneimine in purification of DNA-binding proteins. Methods Enzymol. 208, 3-10 (1991).

99 Lima, C. D., Wang, J. C. \& Mondragon, A. Crystallization of a $67 \mathrm{kDa}$ fragment of Escherichia coli DNA topoisomerase I. J Mol Biol 232, 1213-1216, doi:10.1006/jmbi.1993.1474 (1993).

100 Lima, C. D., Wang, J. C. \& Mondragon, A. Three-dimensional structure of the $67 \mathrm{~K}$ N-terminal fragment of E. coli DNA topoisomerase I. Nature 367, 138-146, doi:10.1038/367138a0 (1994).

101 Hansen, G., Harrenga, A., Wieland, B., Schomburg, D. \& Reinemer, P. Crystal structure of full length topoisomerase I from Thermotoga maritima. J Mol Biol 358, 1328-1340, doi:10.1016/j.jmb.2006.03.012 (2006).

102 Viard, T., Lamour, V., Duguet, M. \& Bouthier de la Tour, C. Hyperthermophilic topoisomerase I from Thermotoga maritima. A very efficient enzyme that functions independently of zinc binding. J Biol Chem 276, 46495-46503, doi:10.1074/jbc.M107714200 (2001).

$103 \mathrm{Xu}, \mathrm{X}$. \& Leng, F. A rapid procedure to purify Escherichia coli DNA topoisomerase I. Protein Expr. Purif. 77, 214-219, doi:10.1016/j.pep.2011.02.002 (2011).

104 Strahs, D., Zhu, C. X., Cheng, B., Chen, J. \& Tse-Dinh, Y. C. Experimental and computational investigations of Ser10 and Lys 13 in the binding and cleavage of DNA substrates by Escherichia coli DNA topoisomerase I. Nucleic Acids Res 34, 1785-1797, doi:10.1093/nar/gk1109 (2006).

105 Lemos, J. A., Quivey, R. G., Jr., Koo, H. \& Abranches, J. Streptococcus mutans: a new Gram-positive paradigm? Microbiology 159, 436-445, doi:10.1099/mic.0.066134-0 (2013). 
106 Studier, F. W. Stable expression clones and auto-induction for protein production in E. coli. Methods Mol Biol 1091, 17-32, doi:10.1007/978-1-62703-691-7_2 (2014).

107 Pappin, D. J., Hojrup, P. \& Bleasby, A. J. Rapid identification of proteins by peptide-mass fingerprinting. Curr. Biol. 3, 327-332 (1993).

108 Henzel, W. J. et al. Identifying proteins from two-dimensional gels by molecular mass searching of peptide fragments in protein sequence databases. Proc. Natl. Acad. Sci. U. S. A. 90, 5011-5015 (1993).

109 Mann, M., Hojrup, P. \& Roepstorff, P. Use of mass spectrometric molecular weight information to identify proteins in sequence databases. Biol. Mass Spectrom. 22, 338-345, doi:10.1002/bms.1200220605 (1993).

110 Eswar, N. et al. Comparative protein structure modeling using MODELLER. Curr Protoc Protein Sci Chapter 2, Unit 2 9, doi:10.1002/0471140864.ps0209s50 (2007).

111 Eswar, N., Eramian, D., Webb, B., Shen, M. Y. \& Sali, A. Protein structure modeling with MODELLER. Methods Mol Biol 426, 145-159, doi:10.1007/9781-60327-058-8 8 (2008).

112 Otwinowski, Z. \& Minor, W. Processing of X-ray diffraction data collected in oscillation mode. Methods Enzymol 276, 307-326 (1997).

113 Cowtan, K. The Buccaneer software for automated model building. 1. Tracing protein chains. Acta Crystallogr D Biol Crystallogr 62, 1002-1011, doi:10.1107/S0907444906022116 (2006).

114 Adams, P. D. et al. PHENIX: a comprehensive Python-based system for macromolecular structure solution. Acta Crystallogr D Biol Crystallogr 66, 213 221, doi:10.1107/S0907444909052925 (2010).

115 Emsley, P., Lohkamp, B., Scott, W. G. \& Cowtan, K. Features and development of Coot. Acta Crystallogr D Biol Crystallogr 66, 486-501, doi:10.1107/S0907444910007493 (2010).

116 Joosten, R. P. et al. PDB_REDO: automated re-refinement of X-ray structure models in the PDB. J Appl Crystallogr 42, 376-384, doi:10.1107/S0021889809008784 (2009).

117 Chen, V. B. et al. MolProbity: all-atom structure validation for macromolecular crystallography. Acta Crystallogr D Biol Crystallogr 66, 12-21, doi:10.1107/S0907444909042073 (2010).

118 Pettersen, E. F. et al. UCSF Chimera--a visualization system for exploratory research and analysis. J Comput Chem 25, 1605-1612, doi:10.1002/jcc.20084 (2004).

119 Robert, X. \& Gouet, P. Deciphering key features in protein structures with the new ENDscript server. Nucleic Acids Res 42, W320-324, doi:10.1093/nar/gku316 (2014).

120 Madeira, F. et al. The EMBL-EBI search and sequence analysis tools APIs in 2019. Nucleic Acids Res, doi:10.1093/nar/gkz268 (2019).

121 Laskowski, R. A., Jablonska, J., Pravda, L., Varekova, R. S. \& Thornton, J. M. PDBsum: Structural summaries of PDB entries. Protein Sci 27, 129-134, doi:10.1002/pro.3289 (2018). 
122 Chojnacki, S., Cowley, A., Lee, J., Foix, A. \& Lopez, R. Programmatic access to bioinformatics tools from EMBL-EBI update: 2017. Nucleic Acids Res 45, W550W553, doi:10.1093/nar/gkx273 (2017).

123 Studier, F. W. \& Moffatt, B. A. Use of bacteriophage T7 RNA polymerase to direct selective high-level expression of cloned genes. J Mol Biol 189, 113-130 (1986).

124 Ferrer, M., Chernikova, T. N., Yakimov, M. M., Golyshin, P. N. \& Timmis, K. N. Chaperonins govern growth of Escherichia coli at low temperatures. Nat. Biotechnol. 21, 1266-1267, doi:10.1038/nbt1103-1266 (2003).

125 Ferrer, M. et al. Functional consequences of single:double ring transitions in chaperonins: life in the cold. Mol. Microbiol. 53, 167-182, doi:10.1111/j.13652958.2004.04077.x (2004).

126 Bradford, M. M. A rapid and sensitive method for the quantitation of microgram quantities of protein utilizing the principle of protein-dye binding. Anal Biochem 72, 248-254 (1976).

127 Ericsson, U. B., Hallberg, B. M., Detitta, G. T., Dekker, N. \& Nordlund, P. Thermofluor-based high-throughput stability optimization of proteins for structural studies. Anal Biochem 357, 289-298, doi:10.1016/j.ab.2006.07.027 (2006).

128 Matthews, B. W. Solvent content of protein crystals. J Mol Biol 33, 491-497 (1968).

129 Kantardjieff, K. A. \& Rupp, B. Matthews coefficient probabilities: Improved estimates for unit cell contents of proteins, DNA, and protein-nucleic acid complex crystals. Protein Sci 12, 1865-1871, doi:10.1110/ps.0350503 (2003).

130 Weichenberger, C. X. \& Rupp, B. Ten years of probabilistic estimates of biocrystal solvent content: new insights via nonparametric kernel density estimate. Acta Crystallogr D Biol Crystallogr 70, 1579-1588, doi:10.1107/S1399004714005550 (2014).

131 Krissinel, E. \& Henrick, K. Inference of macromolecular assemblies from crystalline state. J Mol Biol 372, 774-797, doi:10.1016/j.jmb.2007.05.022 (2007).

132 Aravind, L., Leipe, D. D. \& Koonin, E. V. Toprim--a conserved catalytic domain in type IA and II topoisomerases, DnaG-type primases, OLD family nucleases and RecR proteins. Nucleic Acids Res 26, 4205-4213, doi:10.1093/nar/26.18.4205 (1998).

133 de Beer, T. A., Berka, K., Thornton, J. M. \& Laskowski, R. A. PDBsum additions. Nucleic Acids Res 42, D292-296, doi:10.1093/nar/gkt940 (2014).

134 Fischer, M., Leech, A. P. \& Hubbard, R. E. Comparative assessment of different histidine-tags for immobilization of protein onto surface plasmon resonance sensorchips. Anal Chem 83, 1800-1807, doi:10.1021/ac103168q (2011).

135 Grisshammer, R. \& Tucker, J. Quantitative evaluation of neurotensin receptor purification by immobilized metal affinity chromatography. Protein Expr Purif 11, 53-60, doi:10.1006/prep.1997.0766 (1997).

136 Marblestone, J. G. et al. Comparison of SUMO fusion technology with traditional gene fusion systems: enhanced expression and solubility with SUMO. Protein Sci 15, 182-189, doi:10.1110/ps.051812706 (2006). 
137 Truong, L. et al. High-level expression, purification, and characterization of Staphylococcus aureus dihydroorotase (PyrC) as a cleavable His-SUMO fusion. Protein Expr Purif 88, 98-106, doi:10.1016/j.pep.2012.11.018 (2013).

138 Berg, O. G. \& Blomberg, C. Association kinetics with coupled diffusion III. Ionic-strength dependence of the lac repressor-operator association. Biophys. Chem. 8, 271-280 (1978).

139 Jones, J. A. et al. Small-molecule inhibition of the C. difficile FAS-II enzyme, FabK, results in selective activity. ACS Chem Biol, doi:10.1021/acschembio.9b00293 (2019, In Press).

140 Gil, F., Calderon, I. L., Fuentes, J. A. \& Paredes-Sabja, D. Clostridioides (Clostridium) difficile infection: current and alternative therapeutic strategies. Future Microbiol 13, 469-482, doi:10.2217/fmb-2017-0203 (2018).

141 Dubberke, E. R. \& Olsen, M. A. Burden of Clostridium difficile on the healthcare system. Clin Infect Dis 55 Suppl 2, S88-92, doi:10.1093/cid/cis335 (2012).

142 Zhu, D., Sorg, J. A. \& Sun, X. Clostridioides difficile Biology: Sporulation, Germination, and Corresponding Therapies for C. difficile Infection. Front Cell Infect Microbiol 8, 29, doi:10.3389/fcimb.2018.00029 (2018).

143 Lessa, F. C., Gould, C. V. \& McDonald, L. C. Current status of Clostridium difficile infection epidemiology. Clin Infect Dis 55 Suppl 2, S65-70, doi:10.1093/cid/cis319 (2012).

144 Antharam, V. C. et al. Intestinal dysbiosis and depletion of butyrogenic bacteria in Clostridium difficile infection and nosocomial diarrhea. J Clin Microbiol 51, 2884-2892, doi:10.1128/JCM.00845-13 (2013).

145 Crawford, T., Huesgen, E. \& Danziger, L. Fidaxomicin: a novel macrocyclic antibiotic for the treatment of Clostridium difficile infection. Am J Health Syst Pharm 69, 933-943, doi:10.2146/ajhp110371 (2012).

146 Tsutsumi, L. S., Owusu, Y. B., Hurdle, J. G. \& Sun, D. Progress in the discovery of treatments for C. difficile infection: A clinical and medicinal chemistry review. Curr Top Med Chem 14, 152-175 (2014).

147 Marsh, J. W. et al. Association of relapse of Clostridium difficile disease with BI/NAP1/027. J. Clin. Microbiol. 50, 4078-4082, doi:10.1128/JCM.02291-12 (2012).

148 Garey, K. W., Sethi, S., Yadav, Y. \& DuPont, H. L. Meta-analysis to assess risk factors for recurrent Clostridium difficile infection. J Hosp Infect 70, 298-304, doi:10.1016/j.jhin.2008.08.012 (2008).

149 Lewis, B. B. et al. Pathogenicity Locus, Core Genome, and Accessory Gene Contributions to Clostridium difficile Virulence. MBio 8, doi:10.1128/mBio.00885-17 (2017).

150 Francino, M. P. Antibiotics and the Human Gut Microbiome: Dysbioses and Accumulation of Resistances. Front. Microbiol. 6, 1543, doi:10.3389/fmicb.2015.01543 (2015).

151 Louie, T. J. et al. Fidaxomicin versus vancomycin for Clostridium difficile infection. N Engl J Med 364, 422-431, doi:10.1056/NEJMoa0910812 (2011).

152 Orenstein, R. Fidaxomicin failures in recurrent Clostridium difficile infection: a problem of timing. Clin Infect Dis 55, 613-614, doi:10.1093/cid/cis495 (2012). 
153 Angelakis, E., Armougom, F., Million, M. \& Raoult, D. The relationship between gut microbiota and weight gain in humans. Future Microbiol 7, 91-109, doi:10.2217/fmb.11.142 (2012).

154 McDonald, L. C. et al. Clinical Practice Guidelines for Clostridium difficile Infection in Adults and Children: 2017 Update by the Infectious Diseases Society of America (IDSA) and Society for Healthcare Epidemiology of America (SHEA). Clin Infect Dis 66, e1-e48, doi:10.1093/cid/cix 1085 (2018).

155 Petrosillo, N., Granata, G. \& Cataldo, M. A. Novel Antimicrobials for the Treatment of Clostridium difficile Infection. Front. Med. (Lausanne) 5, 96, doi:10.3389/fmed.2018.00096 (2018).

156 Pedrido, M. E. et al. Spo0A links de novo fatty acid synthesis to sporulation and biofilm development in Bacillus subtilis. Mol Microbiol 87, 348-367, doi:10.1111/mmi.12102 (2013).

157 Heath, R. J. \& Rock, C. O. Fatty acid biosynthesis as a target for novel antibacterials. Curr Opin Investig Drugs 5, 146-153 (2004).

158 Parsons, J. B. et al. Perturbation of Staphylococcus aureus gene expression by the enoyl-acyl carrier protein reductase inhibitor AFN-1252. Antimicrob Agents Chemother 57, 2182-2190, doi:10.1128/AAC.02307-12 (2013).

159 Hunt, T., Kaplan, N. \& Hafkin, B. Safety, tolerability and pharmacokinetics of multiple oral doses of AFN-1252 administered as immediate release (IR) tablets in healthy subjects. J Chemother 28, 164-171, doi:10.1179/1973947815Y.0000000075 (2016).

160 Stabler, R. A. et al. Comparative genome and phenotypic analysis of Clostridium difficile 027 strains provides insight into the evolution of a hypervirulent bacterium. Genome biology 10, R102, doi:10.1186/gb-2009-10-9-r102 (2009).

161 Heath, R. J. \& Rock, C. O. A triclosan-resistant bacterial enzyme. Nature 406, 145-146, doi:10.1038/35018162 (2000).

162 Hevener, K. E. et al. Structural characterization of Porphyromonas gingivalis enoyl-ACP reductase II (FabK). Acta Crystallogr F Struct Biol Commun 74, 105 112, doi:10.1107/S2053230X18000262 (2018).

163 Saito, J. et al. Crystal structure of enoyl-acyl carrier protein reductase (FabK) from Streptococcus pneumoniae reveals the binding mode of an inhibitor. Protein Sci 17, 691-699, doi:10.1110/ps.073288808 (2008).

164 Ozawa, T. et al. Phenylimidazole derivatives as specific inhibitors of bacterial enoyl-acyl carrier protein reductase FabK. Bioorg Med Chem 15, 7325-7336, doi:10.1016/j.bmc.2007.08.050 (2007).

165 Kitagawa, H. et al. Phenylimidazole derivatives of 4-pyridone as dual inhibitors of bacterial enoyl-acyl carrier protein reductases FabI and FabK. J Med Chem 50, 4710-4720, doi:10.1021/jm0705354 (2007).

166 Kitagawa, H., Ozawa, T., Takahata, S. \& Iida, M. Phenylimidazole derivatives as new inhibitors of bacterial enoyl-ACP reductase FabK. Bioorg Med Chem Lett 17, 4982-4986, doi:10.1016/j.bmcl.2007.06.040 (2007).

167 Takahata, S. et al. AG205, a novel agent directed against FabK of Streptococcus pneumoniae. Antimicrob Agents Chemother 50, 2869-2871, doi:10.1128/AAC.00270-06 (2006). 
168 Saito, J., Yamada, M., Watanabe, T., Kitagawa, H. \& Takeuchi, Y. Crystallization and preliminary X-ray analysis of enoyl-acyl carrier protein reductase (FabK) from Streptococcus pneumoniae. Acta Crystallogr Sect F Struct Biol Cryst Commun 62, 576-578, doi:10.1107/S1744309106017039 (2006).

169 White, S. W., Zheng, J., Zhang, Y. M. \& Rock. The structural biology of type II fatty acid biosynthesis. Annu Rev Biochem 74, 791-831, doi:10.1146/annurev.biochem.74.082803.133524 (2005).

170 Oppermann, U. et al. Short-chain dehydrogenases/reductases (SDR): the 2002 update. Chem Biol Interact 143-144, 247-253 (2003).

171 Brinster, S. et al. Type II fatty acid synthesis is not a suitable antibiotic target for Gram-positive pathogens. Nature 458, 83-86, doi:10.1038/nature07772 (2009).

172 Balemans, W. et al. Essentiality of FASII pathway for Staphylococcus aureus. Nature 463, E3; discussion E4, doi:10.1038/nature08667 (2010).

173 Brinster, S. et al. Brinster et al. reply. Nature 463, E4-E5 (2010).

174 Goldfine, H. \& Johnston, N. C. in Handbook on Clostridia (ed P. Duerre) Ch. 15, 297-309 (CRC Press, 2004).

175 Guan, Z., Katzianer, D., Zhu, J. \& Goldfine, H. Clostridium difficile contains plasmalogen species of phospholipids and glycolipids. Biochimica et biophysica acta 1842, 1353-1359, doi:10.1016/j.bbalip.2014.06.011 (2014).

176 Arumugam, M. et al. Enterotypes of the human gut microbiome. Nature 473, 174180, doi:10.1038/nature09944 (2011).

177 Zheng, C. J., Sohn, M. J., Lee, S. \& Kim, W. G. Meleagrin, a new FabI inhibitor from Penicillium chryosogenum with at least one additional mode of action. PloS one 8, e78922, doi:10.1371/journal.pone.0078922 (2013).

178 Yao, J., Ericson, M. E., Frank, M. W. \& Rock, C. O. Enoyl-Acyl Carrier Protein Reductase I (FabI) Is Essential for the Intracellular Growth of Listeria monocytogenes. Infect Immun 84, 3597-3607, doi:10.1128/IAI.00647-16 (2016).

179 Kimple, M. E., Brill, A. L. \& Pasker, R. L. Overview of affinity tags for protein purification. Curr Protoc Protein Sci 73, Unit 9 9, doi:10.1002/0471140864.ps0909s73 (2013).

180 Brooks, H. B. et al. in Assay Guidance Manual (eds G. S. Sittampalam et al.) (2004).

181 Heras, B. \& Martin, J. L. Post-crystallization treatments for improving diffraction quality of protein crystals. Acta Crystallogr D Biol Crystallogr 61, 1173-1180, doi:10.1107/S0907444905019451 (2005).

182 Carson, M., Johnson, D. H., McDonald, H., Brouillette, C. \& Delucas, L. J. Histag impact on structure. Acta Crystallogr D Biol Crystallogr 63, 295-301, doi:10.1107/S0907444906052024 (2007).

183 Kitagawa, H. et al. Phenylimidazole derivatives of 4-pyridone as dual inhibitors of bacterial enoyl-acyl carrier protein reductases FabI and FabK. J. Med. Chem. 50, 4710-4720, doi:10.1021/jm0705354 (2007).

184 Ozawa, T. et al. Phenylimidazole derivatives as specific inhibitors of bacterial enoyl-acyl carrier protein reductase FabK. Biorg. Med. Chem. 15, 7325-7336, doi:10.1016/j.bmc.2007.08.050 (2007). 
185 Takahata, S. et al. AG205, a novel agent directed against FabK of Streptococcus pneumoniae. Antimicrob. Agents Chemother. 50, 2869-2871, doi:10.1128/AAC.00270-06 (2006).

186 Marreddy, R. K. R. et al. The Fatty Acid Synthesis Protein Enoyl-ACP Reductase II (FabK) is a Target for Narrow-Spectrum Antibacterials for Clostridium difficile Infection. ACS Infect. Dis. 5, 208-217, doi:10.1021/acsinfecdis.8b00205 (2019).

187 Zhang, Y. M., Lu, Y. J. \& Rock, C. O. The reductase steps of the type II fatty acid synthase as antimicrobial targets. Lipids 39, 1055-1060 (2004).

188 Marrakchi, H., Zhang, Y. M. \& Rock, C. O. Mechanistic diversity and regulation of Type II fatty acid synthesis. Biochem Soc Trans 30, 1050-1055, doi:10.1042/ (2002).

189 Heath, R. J. et al. Mechanism of triclosan inhibition of bacterial fatty acid synthesis. J Biol Chem 274, 11110-11114 (1999).

190 Parsons, J. B., Frank, M. W., Subramanian, C., Saenkham, P. \& Rock, C. O. Metabolic basis for the differential susceptibility of Gram-positive pathogens to fatty acid synthesis inhibitors. Proceedings of the National Academy of Sciences of the United States of America 108, 15378-15383, doi:10.1073/pnas.1109208108 (2011).

191 Mehboob, S. et al. Structural and biological evaluation of a novel series of benzimidazole inhibitors of Francisella tularensis enoyl-ACP reductase (FabI). Bioorg Med Chem Lett 25, 1292-1296, doi:10.1016/j.bmcl.2015.01.048 (2015).

192 Hevener, K. E. et al. Discovery of a novel and potent class of F. tularensis enoylreductase (FabI) inhibitors by molecular shape and electrostatic matching. Journal of Medicinal Chemistry 55, 268-279, doi:10.1021/jm201168g (2012).

193 Mehboob, S. et al. Structural and Enzymatic Analyses Reveal the Binding Mode of a Novel Series of Francisella tularensis Enoyl Reductase (FabI) Inhibitors. Journal of Medicinal Chemistry 55, 5933-5941, doi:10.1021/jm300489v (2012).

194 Schiebel, J. et al. Staphylococcus aureus FabI: inhibition, substrate recognition, and potential implications for in vivo essentiality. Structure 20, 802-813, doi:10.1016/j.str.2012.03.013 (2012).

195 Liu, N., Cummings, J. E., England, K., Slayden, R. A. \& Tonge, P. J. Mechanism and inhibition of the FabI enoyl-ACP reductase from Burkholderia pseudomallei. $J$ Antimicrob Chemother 66, 564-573, doi:10.1093/jac/dkq509 (2011).

196 Sampson, P. B. et al. Spiro-naphthyridinone piperidines as inhibitors of S. aureus and E. coli enoyl-ACP reductase (FabI). Bioorg Med Chem Lett 19, 5355-5358, doi:10.1016/j.bmcl.2009.07.129 (2009).

197 Ramnauth, J. et al. 2,3,4,5-Tetrahydro-1H-pyrido[2,3-b and e][1,4]diazepines as inhibitors of the bacterial enoyl ACP reductase, FabI. Bioorg Med Chem Lett 19, 5359-5362, doi:10.1016/j.bmcl.2009.07.094 (2009).

198 Zheng, C. J., Sohn, M. J. \& Kim, W. G. Vinaxanthone, a new FabI inhibitor from Penicillium sp. J Antimicrob Chemother 63, 949-953, doi:10.1093/jac/dkp058 (2009).

199 Lu, H. \& Tonge, P. J. Inhibitors of FabI, an enzyme drug target in the bacterial fatty acid biosynthesis pathway. Accounts of chemical research 41, 11-20, doi:10.1021/ar700156e (2008). 
200 Dhillon, G. S. et al. Triclosan: Current Status, Occurrence, Environmental Risks and Bioaccumulation Potential. International journal of environmental research and public health 12, 5657-5684, doi:10.3390/ijerph120505657 (2015).

201 McMurry, L. M., Oethinger, M. \& Levy, S. B. Triclosan targets lipid synthesis. Nature 394, 531-532, doi:10.1038/28970 (1998).

202 Hevener, K. E. et al. Structural characterization of Porphyromonas gingivalis enoyl-ACP reductase II (FabK). Acta Crystallogr. F Struct. Biol. Commun. 74, 105-112, doi:10.1107/S2053230X18000262 (2018).

203 Priyadarshi, A., Kim, E. E. \& Hwang, K. Y. Structural insights into Staphylococcus aureus enoyl-ACP reductase (FabI), in complex with NADP and triclosan. Proteins 78, 480-486, doi:10.1002/prot.22581 (2010).

204 Kingry, L. C. et al. The Francisella tularensis FabI enoyl-acyl carrier protein reductase gene is essential to bacterial viability and is expressed during infection. J Bacteriol 195, 351-358, doi:10.1128/JB.01957-12 (2013).

205 Khan, R. et al. Biochemical and Structural Basis of Triclosan Resistance in a Novel Enoyl-Acyl Carrier Protein Reductase. Antimicrob. Agents Chemother. 62, pii: e00648-00618, doi:10.1128/AAC.00648-18 (2018).

206 Kanehisa, M., Sato, Y., Furumichi, M., Morishima, K. \& Tanabe, M. New approach for understanding genome variations in KEGG. Nucleic Acids Res 47, D590-D595, doi:10.1093/nar/gky962 (2019).

207 Kanehisa, M., Furumichi, M., Tanabe, M., Sato, Y. \& Morishima, K. KEGG: new perspectives on genomes, pathways, diseases and drugs. Nucleic Acids Res 45, D353-D361, doi:10.1093/nar/gkw1092 (2017).

208 Kanehisa, M. \& Goto, S. KEGG: kyoto encyclopedia of genes and genomes. Nucleic Acids Res 28, 27-30, doi:10.1093/nar/28.1.27 (2000).

209 Wilkins, M. R. et al. Protein identification and analysis tools in the ExPASy server. Methods Mol Biol 112, 531-552 (1999).

210 Spagnuolo, L. A. et al. Evaluating the Contribution of Transition-State Destabilization to Changes in the Residence Time of Triazole-Based InhA Inhibitors. J Am Chem Soc 139, 3417-3429, doi:10.1021/jacs.6b11148 (2017).

211 Copeland, R. A. Conformational adaptation in drug-target interactions and residence time. Future Med Chem 3, 1491-1501, doi:10.4155/fmc.11.112 (2011).

$212 \mathrm{Li}, \mathrm{H}$. J. et al. A structural and energetic model for the slow-onset inhibition of the Mycobacterium tuberculosis enoyl-ACP reductase InhA. ACS Chem Biol 9, 986-993, doi:10.1021/cb400896g (2014).

213 Swinney, D. C. et al. A study of the molecular mechanism of binding kinetics and long residence times of human CCR5 receptor small molecule allosteric ligands. Br J Pharmacol 171, 3364-3375, doi:10.1111/bph.12683 (2014).

214 Niedzialkowska, E. et al. Protein purification and crystallization artifacts: The tale usually not told. Protein Sci 25, 720-733, doi:10.1002/pro.2861 (2016).

215 Derewenda, Z. S. Application of protein engineering to enhance crystallizability and improve crystal properties. Acta Crystallogr D Biol Crystallogr 66, 604-615, doi:10.1107/S090744491000644X (2010).

216 Holcomb, J. et al. Protein crystallization: Eluding the bottleneck of X-ray crystallography. AIMS Biophys 4, 557-575, doi:10.3934/biophy.2017.4.557 (2017). 
217 Roy, A., McDonald, P. R., Sittampalam, S. \& Chaguturu, R. Open access high throughput drug discovery in the public domain: a Mount Everest in the making. Curr Pharm Biotechnol 11, 764-778 (2010).

218 Dahlin, J. L., Inglese, J. \& Walters, M. A. Mitigating risk in academic preclinical drug discovery. Nat Rev Drug Discov 14, 279-294, doi:10.1038/nrd4578 (2015).

219 Simeonov, A. \& Davis, M. I. in Assay Guidance Manual (eds G. S. Sittampalam et al.) (2004).

220 Bray, M. A. \& Carpenter, A. in Assay Guidance Manual (eds G. S. Sittampalam et al.) (2004).

221 Zhang, J. H., Chung, T. D. \& Oldenburg, K. R. A Simple Statistical Parameter for Use in Evaluation and Validation of High Throughput Screening Assays. $J$ Biomol Screen 4, 67-73, doi:10.1177/108705719900400206 (1999).

222 Ursu, O., Glick, M. \& Oprea, T. Novel drug targets in 2018. Nat Rev Drug Discov, doi:10.1038/d41573-019-00052-5 (2019).

223 Morrow, T. \& Felcone, L. H. Defining the difference: What Makes Biologics Unique. Biotechnol Healthc 1, 24-29 (2004). 


\section{VITA}

Jesse Arin Jones was born in Blackfoot, Idaho to Rose and Larry Jones in 1983. He graduated from Skyline High School in Idaho Falls, Idaho in 2002. Afterward, he enrolled at Boise State University, where he received a Bachelor of Science in Political Science, with a minor in Chemistry in May 2008. Later that fall, he entered pharmacy school at Idaho State University. He completed his doctorate of pharmacy in May of 2012 and began practicing pharmacy in the Boise, Idaho area. In the Fall of 2013, he returned to Idaho State University and enrolled in the $\mathrm{PhD}$ program. After three and a half years, he transferred to the University of Tennessee Health Science Center with his $\mathrm{PhD}$ advisor to finish his research and degree. He completed his Pharmaceutical Sciences doctorate in August 2019. 